
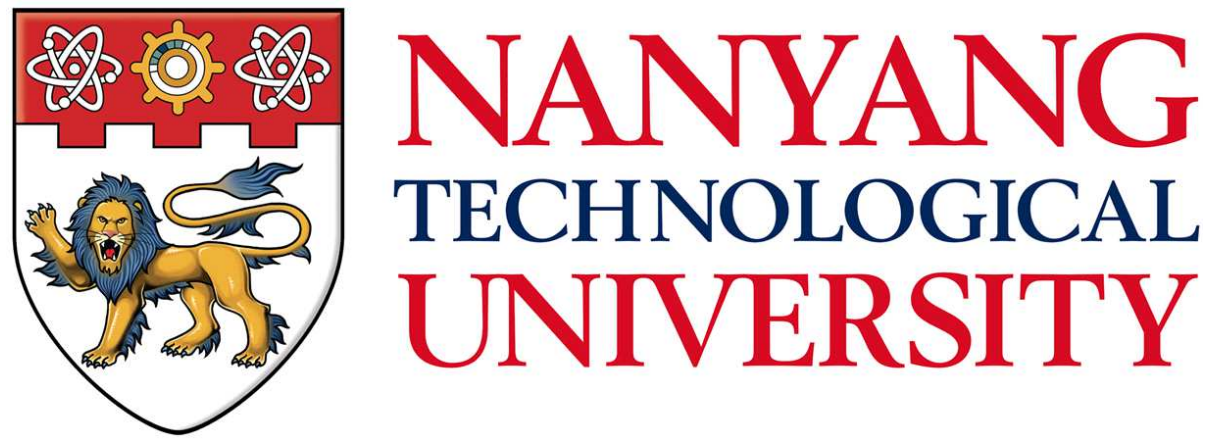

\title{
A PRINTED HYBRID WARM WHITE LIGHT SOURCE: FROM FABRICATION TO PHYSICS-BASED MODEL
}

ZHANG SHUAI

SCHOOL OF ELECTRICAL AND ELECTRONIC ENGINEERING 


\section{A PRINTED HYBRID WARM WHITE LIGHT SOURCE: FROM FABRICATION TO PHYSICS-BASED MODEL}

\section{ZHANG SHUAI}

School of Electrical and Electronic Engineering

A thesis submitted to the Nanyang Technological Univeristy in partial fulfilment of the requirement for the degree of Doctor of Philosophy 
For God and my family. 


\section{Acknowledgments}

It has been a challenging and fruitful four years' journey with part-time study and fulltime working experience. Without many individuals I could not go this far. First and foremost, I would like to thank my PhD supervisor Associate Professor Wong Kin Shun, Terence for his patient guidance, encouragement and support. During the transfer from another group at the end of my second year, Prof Wong encouraged me to hold on and stay focused on the little findings. I am sincerely thankful for his inspirational thoughts on the selection of research topic and polishing of the motivations and background. I also appreciate his step-by-step guidance on experimental details and writing manuscripts. I am deeply grateful for his time and effort in helping me on every milestone such as QE, yearly update report, and this thesis preparation.

Secondly, I also want to thank my co-supervisor Associate Professor Su Haibin. Prof $\mathrm{Su}$ has always been a listener and friend more than just a mentor to me since the final year of my undergraduate study. I am very grateful for his care and understanding on my academic and personal life. I want to thank him especially for the advise in the modelling part of this thesis. I also appreciate his time and effort in helping with the publication of research papers.

In many ways, this study would not be possible without the support from Dr Teo Jin Wah, Ronnie from Singapore Institute of Manufacturing Technology (SIMTech). I would like to thank him for the financial support for these five years' working experience in SIMTech-NTU joint lab on reliability. I am also deeply grateful and touched by his endless guidance and detailed advice on every experimental plan. I also appreciate for his efforts on helping my personal life and developing my character and personality.

My sincere thanks also go to Associate Professor Tan Chuan Seng for his administrative support on the projects of this study and smooth transition from last project. I also want 
to thank my colleague $\mathrm{Mr} \mathrm{Yu}$ Wenzhi for his help in preparing samples and setting up characterization systems. I would also like to thank Mr Lee Chew Siong, Thomas, Mr Wee Seng Khoon, Mr Ng Yoon Chiang, and Mr Chua Tong Sun for their laboratory and technical help and guidance in work safety and the usage of characterization equipment.

I wish to thank all the staffs in the LAP team in SIMTech for providing the materials and facilities to carry out printing processes and for their sharing and teaching of technical knowledge. I would especially thank Mr Lim Lay Huat and Mr Uvarajan S/O M Velayutham from SIMTech for their help in conducting optical and thermal measurement.

Finally, I would like to thank my parents for their love and support. Particularly, I want to thank my wife for her support, love, companion and hard works even during her pregnancy. I want to thank God for His grace and mercy so that I have the strength to keep on and come so far to this stage. 


\section{Table of Contents}

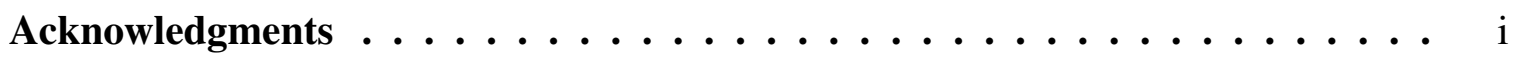

Table of Contents $\ldots \ldots \ldots \ldots \ldots \ldots \ldots$ iii

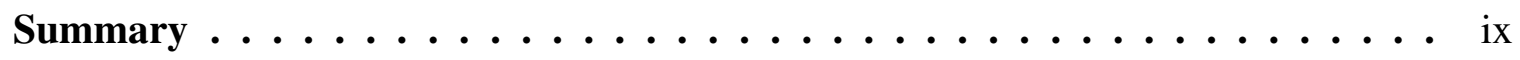

List of Publications $\ldots \ldots \ldots \ldots \ldots \ldots \ldots \ldots \ldots$

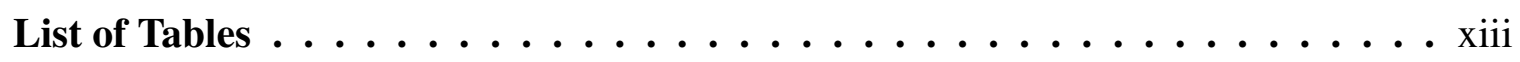

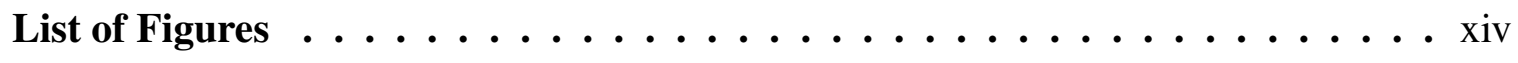

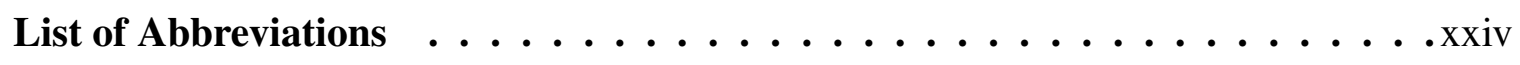

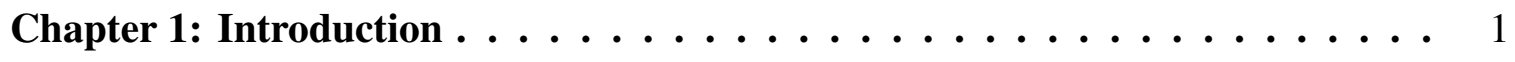

1.1 Problem statement and motivation $\ldots \ldots \ldots \ldots$

1.2 Scope and objectives ......................... 2

1.3 Significance of the research $\ldots \ldots \ldots \ldots$

1.4 Organization of thesis $\ldots \ldots \ldots \ldots \ldots$

Chapter 2: Literature Review ................... 5

2.1 ACPEL devices ....................... 5

2.1.1 History of ACPEL devices .............. 5

2.1.2 Device architectures ............... 6 
2.1.3 Materials for forward and reversed ACPEL devices . . . . . . . . 8

2.1.4 Properties of $\mathrm{ZnS}$ and its phosphor powders . . . . . . . . . 13

2.1.5 Light emission mechanisms _. . . . . . . . . . . . . . . . 19

2.2 Printing and coating processes ................... 23

2.2.1 Drop casting . . . . . . . . . . . . . . . . 24

2.2.2 Flexography . . . . . . . . . . . . . . . . . 24

2.2 .3 Screen printing .................... 25

2.2 .4 Spin coating . . . . . . . . . . . . . . . 27

2.2.5 blade coating . . . . . . . . . . . . . . . . 29

2.3 Optical radiation metrology . . . . . . . . . . . . . 30

2.3.1 Luminance . . . . . . . . . . . . . . . . . 30

2.3.2 Luminous efficacy and efficiency . . . . . . . . . . . 31

2.3.3 CIE chromaticity ................... 31

2.3.4 Color correlated temperature . . . . . . . . . . . . 33

2.3.5 Color rendering index . . . . . . . . . . . . . . . 34

2.4 Dielectric impedance spectroscopy . . . . . . . . . . . . . 36

2.4.1 Fundamentals of impedance spectroscopy . . . . . . . . . . . 36

2.4.2 Dielectric properties of resin . . . . . . . . . . . . 44

2.4.3 Dielectric properties of polymer composites . . . . . . . . . . . 45

2.5 Summary of research gaps $\ldots \ldots \ldots$. . . . . . . . . . . 50

Chapter 3: Printed hybrid EL films by drop casting process . . . . . . . . . . . 51

3.1 Introduction . . . . . . . . . . . . . . 51 


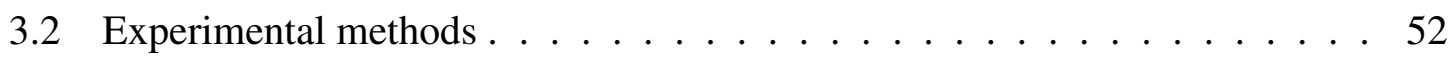

3.2.1 Fabrication of forward and reversed EL films $\ldots \ldots \ldots$. . . . 52

3.2.2 Selection of dye, solvent and coating method . . . . . . . . 54

3.2.3 Drop casting of dye-chloroform solution . . . . . . . . . . 55

3.2.4 Fabrication of white hybrid EL films . . . . . . . . . . 56

3.2.5 Characterization methods . . . . . . . . . . . . . . . . . 57

3.3 Results and discussion $\ldots \ldots \ldots \ldots$

3.3.1 Forward and reversed EL films . . . . . . . . . . . . . . . 59

3.3.2 Solvent effect and spin coating method . . . . . . . . . . 63

3.3.3 Drop casting of a single-dye solution . . . . . . . . . . 65

3.3.4 Drop casting of F8BT and DCJTB mixed solution . . . . . . . . 69

3.3 .5 Aging study $\ldots \ldots \ldots \ldots \ldots \ldots \ldots \ldots$

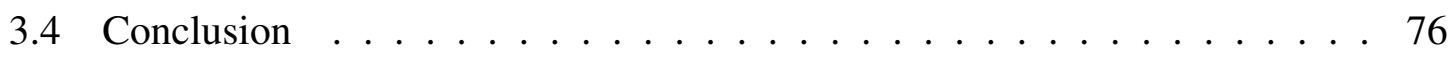

Chapter 4: Printed hybrid EL films by wet stamping and bar coating processes . 78

4.1 Introduction $\ldots \ldots \ldots \ldots \ldots \ldots \ldots \ldots \ldots$

4.2 Experimental details . . . . . . . . . . . . . . . 78

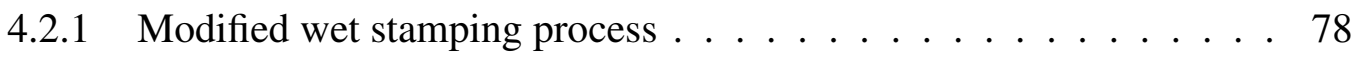

4.2.2 Optimization of wet stamping conditions using fluorescein $\ldots$. . 79

4.2.3 Fabrication of white EL films with DCJTB and fluorescein mixture by wet stamping process . . . . . . . . . . . 81

4.2.4 Fabrication of warm white hybrid films by wet stamping process $\ldots 81$

4.2.5 Fabrication of large-area warm white hybrid films by bar coating technique . . . . . . . . . . . . . 82 
4.2.6 Device measurement and characterization . . . . . . . . . . 83

4.3 Results and discussion $\ldots \ldots \ldots \ldots$. . . . . . . . . . . . 84

4.3.1 Optimization of wet stamping conditions . . . . . . . . . . . . . 84

4.3.2 Wet stamped hybrid films with DCJTB and fluorescein . . . . . 85

4.3.3 Optimization of bar coating conditions . . . . . . . . . . . 92

4.3.4 Bar coated hybrid films . . . . . . . . . . . . . . 96

4.3.5 Aging study . . . . . . . . . . . . . . . . . 100

4.4 Conclusions . . . . . . . . . . . . . . . . . . . 102

Chapter 5: Modeling of frequency-dependent color shift . . . . . . . . . . . . 104

5.1 Introduction . . . . . . . . . . . . . . . . . 104

5.2 Experimental methods . . . . . . . . . . . . . . 105

5.3 Experimental results . . . . . . . . . . . . . . 105

5.3.1 Voltage effects .................... 105

5.3.2 Frequency effects ................... 106

5.4 Previous models . . . . . . . . . . . . . . . . . . . 112

5.5 Proposed model . . . . . . . . . . . . . . . . . 115

5.5.1 Excitation mechanism . . . . . . . . . . . 116

5.5.2 Luminescent centers and recombination mechanisms . . . . . . 118

5.5.3 Charge transport and relaxation model . . . . . . . . . . . . . 124

5.5.4 Analytical solutions . . . . . . . . . . . . . . . 127

5.6 Discussion . . . . . . . . . . . . . . . . . . . 129

5.7 Conclusion . . . . . . . . . . . . . . . . 131 
Chapter 6: Temperature-dependent impedance spectroscopy of ACPEL devices 133

6.1 Introduction . . . . . . . . . . . . . . . 133

6.2 Experimental methods . . . . . . . . . . . . . . . . . . 134

6.2.1 Fabrication of samples for impedance spectroscopy . . . . . . . . . 134

6.2.2 Setup for temperature controlled impedance spectroscopy . . . . . . 135

6.3 Results and discussion $\ldots \ldots \ldots \ldots$. . . . . . . . . . . . . . . . . . .

6.3.1 Temperature dependent relative Permittivity . . . . . . . . . 136

6.3.2 Temperature dependent electric modulus . . . . . . . . . . . . . 139

6.3.3 Temperature dependent conductivity . . . . . . . . . . . . 147

6.3 .4 Equivalent circuit . . . . . . . . . . . . . . . . . 149

6.4 Conclusion . . . . . . . . . . . . . . . . 151

Chapter 7: Opto-impedance spectroscopy of ACPEL devices . . . . . . . . 153

7.1 Introduction . . . . . . . . . . . . . . . 153

7.2 Experimental methods . . . . . . . . . . . . . . . . 154

7.2.1 Experiment setup . . . . . . . . . . . . . . 154

7.2.2 Experiment procedure . . . . . . . . . . . . 155

7.3 Results and discussion $\ldots \ldots . \ldots \ldots 6$

7.3.1 Wavelength effects ...................... 156

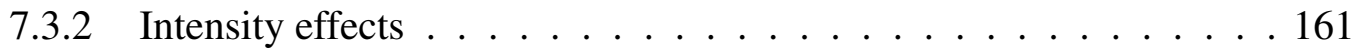

7.3.3 Equivalent circuit analysis . . . . . . . . . . . . 166

7.3.4 Opto-impedance mechanism . . . . . . . . . . . . 169

7.4 Conclusion . . . . . . . . . . . . . . . 170 
Chapter 8: Conclusions and future works . . . . . . . . . . . . . . 172

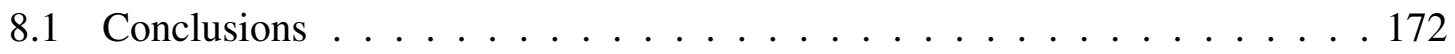

8.2 Future works . . . . . . . . . . . . . . . . . . . 174

References . . . . . . . . . . . . . . . . . . . . . 194 


\begin{abstract}
With the emerging of printed flexible and stretchable devices, a warm white light source is desirable to minimize the ecological impacts and human health issues brought by light emitting diodes (LEDs). Drop casting, wet stamping and bar coating techniques in combination with screen printing process are implemented to fabricate electroluminescence (EL) films hybrid with downshifting organic dyes. Various solutions utilizing green to red organic dyes such as F8BT, fluorescein, DCJTB and DCM2 are formulated with solvents (chloroform and ethanol). With the optimization of process conditions and design of experiments on solution formulation, a hybrid white EL film with CIE of $(0.313,0.312)$ is obtained by drop casting and wet stamping processes. With the varying of number of coated layers, warm white hybrid films with the lowest correlated color temperature (CCT) of $2820 \mathrm{~K}$ and the highest luminance of $89.3 \mathrm{~cd} / \mathrm{m}^{2}$ are achieved using the wet stamping and bar coating processes. However, the hybrid warm white EL film has a luminous efficacy of only $0.5 \mathrm{~lm} / \mathrm{W}$.
\end{abstract}

To understand the root cause of this low luminous efficacy, two methodologies are approached, phosphor level and device level. The first approach is to study the frequency dependent color shift experimentally and theoretically. The color shift is found to be caused by the change of intensity per cycle of three emission lines, which are decomposed from EL emission spectra. A charge transport and relaxation model is proposed based on the detailed review of the luminescent centers and recombination mechanisms of these emission lines. The charge loss during trapping and recombination process and due to the charge relaxation is proposed to contribute to the low luminous efficacy of the hybrid warm white EL films at the $\mathrm{ZnS}$ phosphor level. The second approach utilizes a novel opto-impedance spectroscopy apparatus and methodology to assess the dielectric relaxation processes within the unexcited and excited AC powder electroluminescence (ACPEL) devices. With the 
construction of an equivalent circuit model representing these relaxation processes, effects of illumination wavelengths and intensities are investigated on ACPEL devices, phosphor and dielectric films. A charge transport and accumulation model is proposed to explain the opto-impedance behaviors. The charges escaping out of $\mathrm{ZnS}$ phosphor are attributed to the low luminous efficacy from the device level. 


\section{List of Publications}

\section{Journal articles:}

1. Shuai Zhang, Ronnie Jin Wah Teo, Chuan Seng Tan, Haibin Su, and Terence Kin Shun Wong, "Large area hybrid warm white light source by low cost printing processes," to be submitted.

2. Shuai Zhang, Ronnie Jin Wah Teo, Chuan Seng Tan, Terence Kin Shun Wong, and Haibin $\mathrm{Su}$, "Charge relexation model of frequency dependent color shift in $\mathrm{ZnS}$ phosphors," to be submitted.

3. Shuai Zhang, Chuan Seng Tan, Terence Kin Shun Wong, Haibin Su, and Ronnie Jin Wah Teo, “Opto-impedance spectroscopy and equivalent circuit analyses of AC powder electroluminescent devices," accepted in Optics Express.

4. Shuai Zhang, Haibin Su, Chuan Seng Tan, Terence Kin Shun Wong, and Ronnie Jin Wah Teo, "Dielectric relaxation in AC powder electroluminescent devices," Solid State Communications, 250, 53-56 (2017).

5. Shuai Zhang, Ronnie Jin Wah Teo, Haibin Su, Chuan Seng Tan, and Terence Kin Shun Wong, "Color tunable hybrid AC powder electroluminescent devices with organic fluorescent materials," Optical Materials Express, 6, 2879-2891 (2016).

\section{Technical Disclosure:}

1. Shuai Zhang, Chuan Seng Tan, Terence Kin Shun Wong, and Ronnie Jin Wah Teo, “Apparatus and method for opto-impedance spectroscopy”, Sim/Z/09668, 2016.

\section{Conference presentations:}


1. Shuai Zhang, Ronnie Jin Wah Teo, Haibin Su, Chuan Seng Tan, and Terence Kin Shun Wong, "White light emission from hybrid AC powder electroluminescent devivces," 26th Asian Session Advanced Metallization Conference, Tokyo, Japan, 2016.

2. Shuai Zhang, and Terence Kin Shun Wong, "Modification of AC electroluminescence from $\mathrm{ZnS}: \mathrm{Cu}$ powder phosphors by organic down-conversion materials," International Union of Materials Research Societies International Conference on Electronic Materials (IUMRS-ICEM), Singapore, 2016. 


\section{List of Tables}

2.1 Pros and Cons for the materials used in each layer of the ACPEL devices. . 10

3.1 Variation of concentration and mass ratio of the dye mixture used for the fabrication of white hybrid ACPEL devices. . . . . . . . . . . 57

4.1 Experimental matrix for the optimization of various experiment conditions. $\quad 80$

4.2 Various mass ratios and concentrations of mixed DCJTB and fluorescein solution. . . . . . . . . . . . . . . . 81

6.1 Activation energy and VTF parameters for ACPEL devivces, dielectric film, phosphor film and IBI. . . . . . . . . . . . . . . . . . . 146

6.2 VTF parameters for conductivities in ACPEL devivces, dielectric film, phosphor film and IBI. . . . . . . . . . . . . . . . . . . 150

7.1 Simulated values of the equivalent circuit components of the ACPEL devices under the $399 \mathrm{~nm}$ illumination of different intensities. . . . . . . . . 167

7.2 Simulated values of the equivalent circuit components of the phosphor film under the $399 \mathrm{~nm}$ illumination of different intensities. . . . . . . . . . . . 167 


\section{List of Figures}

2.1 Architecture of forward ACPEL devices. Light is emitted from the substrate side. . . . . . . . . . . . . . . . . . 7

2.2 Architecture of reversed ACPEL devices. Light is emitted from the front electrode. . . . . . . . . . . . . . . . . . 7

2.3 Architecture of interdigitated ACPEL devices. Light is emitted from phosphors that are within the two parallel electrodes. . . . . . . . . . . . 8

2.4 Architecture of combined ACPEL devices. Light is emitted from the substrates side. . . . . . . . . . . . . . . . . . . . 9

2.5 Binary phase diagram for the Zn-S system [76]. . . . . . . . . . . . . . . . 14

2.6 XRD spectra of $\mathrm{ZnS}: \mathrm{Cu}, \mathrm{Cl}$ with $\mathrm{Cu}$ addition in the range of 40 to $5000 \mathrm{ppm}$ fired at $900{ }^{\circ} \mathrm{C}[78] \ldots \ldots \ldots \ldots$. . . . . . . . . . . . . . . 15

2.7 Observation of Electroluminescent lines in $\mathrm{ZnS}$ Powder Particles. (a) birefringent lines extending parallel to long cavity. Bright area when the device is not excited. (c) $\mathrm{ZnS}$ is excited by an AC electric field . Electroluminescent double line at site of the birefringent striation [45] . . . . . . . . . . 16

2.8 (a) SEM image of a $\mathrm{ZnS}: \mathrm{Cu}$-based particle after etching with hydrochloric acid for $15 \mathrm{~min}$, showing a layered structure [79]. (b) Cross-sectional TEM images of a $\mathrm{ZnS}$ phosphor grain. Twin microstructure is seen at high resolution, together with a narrow intergrowth of wurtzite type indicated by the white arrow [80]. . . . . . . . . . . . . . . . 16

2.9 Schematic band-gap diagram of $\mathrm{ZnS}: \mathrm{Cl}$ showing deep defect levels [85]. . . 17

2.10 Energy levels within the band gap of $\mathrm{ZnS}$ for various dopants and accepts [92].

2.11 (a) Typical microscopic view of EL from $\mathrm{ZnS}: \mathrm{Cu}, \mathrm{Cl}$ particles. (b) Phosphor particles containing dark segregations and emitting spots. Double lines at threshold voltage and above the threshold voltage are illustrated [46] . . . . 19 
2.12 Illustration of the phenomenon of the electric field concentrated on the tips of the conducting needles [46]. . . . . . . . . . . . . . . . . 19

2.13 Illustration of the basic principle of the bipolar field model indicating the trap sites [46] . . . . . . . . . . . . . . . . . . . . . 21

2.14 Band behaviors at the contact between a defective border and the bulk ZnS. Three situations are shown: (a) when no field is applied, (b) when the field is parallel to the border, and (c) when the field is directed in opposite senses [81]. . . . . . . . . . . . . . . . . . 23

2.15 Principle of flexography $[103] \ldots \ldots \ldots \ldots \ldots$

2.16 Principle of screen printing $[103] . \ldots \ldots \ldots \ldots \ldots \ldots$

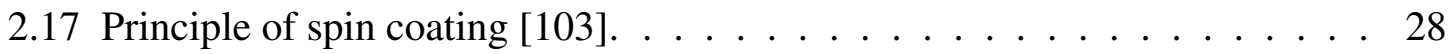

2.18 Principle of blade coating [105]. . . . . . . . . . . . . . . . . . . . . . 29

2.19 (a) dielectric material in a capacitor with randomly distributed dipoles. (b) An electric field is applied to the dielectric and dipoles are aligned. (c) Details of the electric field inside the dielectric. . . . . . . . . . . . . . 37

2.20 Loss tangent versus temperature at different frequencies. The inset shows a magnification region of the $\beta$ relaxation [112]. . . . . . . . . . . 44

2.21 $\mathrm{BaTiO}_{3}$ particle size-dependence of the dielectric constants of the $\mathrm{BaTiO}_{3}$ /PVDF nanocomposites measured at room temperature at $1.14 \mathrm{kHz}$ [134]. . 47

2.22 Dependence of dielectric properties of the composites on the volume concentration of $\mathrm{BaTiO}_{3}(0.1 \mu \mathrm{m}$ and $0.7 \mu \mathrm{m})$ at $10^{3} \mathrm{~Hz}$ measured at room temperature $[141] \ldots \ldots \ldots \ldots \ldots$

2.23 Imaginary part of electric modulus spectra as temperature and frequency for (a) neat epoxy, (b) composite-1 and (c) composite-2; (d) M" vs. temperature at $10^{3} \mathrm{~Hz}$ for all samples $[144] \ldots \ldots \ldots \ldots$. . . . . . . .

3.1 The screen printing process flow for forward ACPEL devices. Device architectures along the process are presented as the insets with the final device structure presented. . . . . . . . . . . . . . . 53

3.2 The screen printing process flow for reversed ACPEL devices. Device architectures along the process are presented as the insets with the final device structure presented. . . . . . . . . . . . . . . . . 54 
3.3 Chemical structures of (a) F8BT and (b) DCJTB. . . . . . . . . . 55

3.4 The drop casting process. RT: room temperature. $\ldots \ldots \ldots \ldots$

3.5 Structures of (a) forward and (b) reversed hybrid ACPEL devices with a layer of drop casted organic dye. . . . . . . . . . . . . 56

3.6 PL emission and excitation spectra of (a) green and (b) blue $\mathrm{ZnS}$ phosphors. 58

3.7 SEM images of (a) $\mathrm{ZnS}$ phosphor and (b) $\mathrm{BaTiO}_{3}$ dielectric powders. . . . . 60

3.8 SEM images of (a) forward and (b) reversed EL films. . . . . . . . . . 60

3.9 (a) EL spectra at $110 \mathrm{~V}$ and $400 \mathrm{~Hz}$ and (b) Commission Internationale de l' clairage (CIE) at $110 \mathrm{~V}$ and different frequencies for Forward Green, Reversed Green, Forward Blue and Reversed Blue EL films. . . . . . . . . 61

3.10 Optical transmittance of ITO-coated PET and PEDOT:PSS. . . . . . . . . . 61

3.11 Luminance as a function of (a) voltage at $400 \mathrm{~Hz}$ and (b) frequency at 110 $\mathrm{V}$ for forward and reversed blue or green EL films. . . . . . . . . . 62

3.12 PL emission and absorbance spectra of (a) F8BT and (b) DCJTB in $\mathrm{CHCl}_{3}$ and on PET films. . . . . . . . . . . . . . . . . . . . . 64

3.13 PL emission spectra of F8BT and DCJTB mixed with (a) binder and (b) PDMS before and after curing. . . . . . . . . . . . . . . 65

3.14 EL spectra of reversed blue EL films with spin-coated dye layer with the mass ratio of F8BT:DCJTB at 9:1 and 1:9 respectively. . . . . . . . . 65

3.15 (a) EL spectra at $110 \mathrm{~V}$ and $400 \mathrm{~Hz}$ and (b) CIE at $110 \mathrm{~V}$ and different frequencies for forward and reversed blue EL films with a layer of F8BT or DCJTB organic dye. . . . . . . . . . . . . . . . 67

3.16 Dark field images of (a) forward F8BT, (b) reversed F8BT, (c) forward DCJTB, and (d) reversed DCJTB operated at $110 \mathrm{~V}$ and $400 \mathrm{~Hz} . ~ \ldots . . .667$

3.17 SEM images of (a)-(c) DCJTB over forward EL films; (d) optical images of drop casted DCJTB layer in reversed DCJTB. . . . . . . . . . . . 68

3.18 (a) EL spectra at $110 \mathrm{~V}$ and $400 \mathrm{~Hz}$ for reversed blue EL films with different mass ratios of F8BT and DCJTB, (b) band diagrams of ZnS, F8BT and DCJTB illustrating the downshifting process. . . . . . . . 70 
3.19 (a) CIE and (b) luminance as a function of frequency at $110 \mathrm{~V}$ for reversed blue EL films with different mass ratios of F8BT and DCJTB. . . . . . . 71

3.20 (a) EL spectra at $110 \mathrm{~V}$ and $400 \mathrm{~Hz}$, (b) luminance and (c) CIE as a function of frequency at $110 \mathrm{~V}$ for reversed blue EL films with different concentrations of $\mathrm{F} 8 \mathrm{BT}$ and DCJTB mixed solution in $\mathrm{CHCl}_{3} \ldots \ldots \ldots . . . .72$

3.21 (a) EL spectra and (b) luminance as a function of time for reversed blue EL films exposed to a constant white light source. . . . . . . . . . . 74

3.22 (a) EL spectra and (b) luminance as a function of time for reversed blue EL films operated at $110 \mathrm{~V}$ and $400 \mathrm{~Hz} . \ldots \ldots \ldots . . \ldots 74$

3.23 (a) EL spectra and (b) luminance as a function of time for reversed hybrid blue EL films with F8BT and DCJTB layer exposed to a constant white light source. . . . . . . . . . . . . . . . . . . 75

3.24 (a) EL spectra and (b) luminance as a function of time for reversed hybrid blue EL films with F8BT and DCJTB layer operated at $110 \mathrm{~V}$ and $400 \mathrm{~Hz}$. 76

4.1 Modified wet stamping process integrated with dip coating and temperature control. RT: room temperature. . . . . . . . . . . . . . . . . 80

4.2 Molecular structures of (a) fluorescein sodium and (b) DCM2 . . . . . 80

4.3 Normalized PL emission and absorbance spectra of (a) DCJTB-ethanol solution and DCJTB-PET film and (b) fluorescein-ethanol solution and fluorescein-PET film. . . . . . . . . . . . . . . . . . 84

4.4 Uniformity of reversed EL films deposited with a layer of fluorescein using wet stamping process with different experimental conditions. . . . . . . 86

4.5 EL spectra at $110 \mathrm{~V}$ and $400 \mathrm{~Hz}$ for hybrid EL films with fixed dye concentration at $2 \mathrm{mg} / \mathrm{mL}$ and different DCJTB:fluorescein mass ratios. . . . . . . 87

4.6 Downshifting mechanism of DCJTB and fluorescein from blue $\mathrm{ZnS}$ phos-

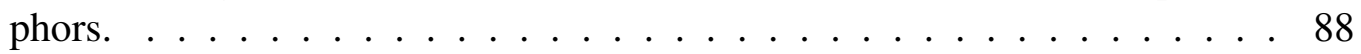

4.7 CIE at $110 \mathrm{~V}$ and 100, 400, 800, 1200, 1600, $2000 \mathrm{~Hz}$ for hybrid EL films with fixed dye concentration at $2 \mathrm{mg} / \mathrm{mL}$ and different DCJTB:fluorescein mass ratios. . . . . . . . . . . . . . . . . . . . . 89 
4.8 (a) EL spectra at $110 \mathrm{~V}$ and $400 \mathrm{~Hz}$ and (b) CIE at $110 \mathrm{~V}$ and 100, 400, $800,1200,1600,2000 \mathrm{~Hz}$ for hybrid EL films with weight of $0.40 \mathrm{~kg}$ or $0.99 \mathrm{~kg}$ and drying time of $10 \mathrm{~s}$ or $15 \mathrm{~s} \ldots \ldots \ldots$. . . . . . . . 89

4.9 (a) EL spectra at $110 \mathrm{~V}$ and $400 \mathrm{~Hz}$ and (b) CIE at $110 \mathrm{~V}$ and 100, 400, 800, 1200, 1600, $2000 \mathrm{~Hz}$ for hybrid EL films with fixed DCJTB:fluorescein mass ratio at $1: 1$ and varied dye concentrations . . . . . . . . . . . 91

4.10 (a) EL spectra at $110 \mathrm{~V}$ and $400 \mathrm{~Hz}$ and (b) CCT as a function of voltage at $400 \mathrm{~Hz}$ for hybrid EL films stamped with one to five layers of DCJTB solution at $4 \mathrm{mg} / \mathrm{mL} \ldots \ldots \ldots$. . . . . . . . . . . . . . . 92

4.11 (a) EL spectra at $110 \mathrm{~V}$ and $400 \mathrm{~Hz}$ and (b) CCT as a function of voltage at $400 \mathrm{~Hz}$ for hybrid EL films stamped with one, two, four and five layers of DCJTB-fluorescein solution at $4 \mathrm{mg} / \mathrm{mL}$ and with the mass ratio of 1:1. Inset: the image showing warm white color of stamped sample operated at $200 \mathrm{~V}$ and $400 \mathrm{~Hz}$.

4.12 PL emission spectrum excited by $450 \mathrm{~nm}$ photons and absorbance spectrum of DCM2 dissolved in ethanol.

4.13 (a) EL spectra at $110 \mathrm{~V}$ and $400 \mathrm{~Hz}$ and (b) CIE at $110 \mathrm{~V}$ and 100, 400, 800, 1200,1600, $2000 \mathrm{~Hz}$ for bar coated hybrid EL films using bars with thread thicknesses of 40,60, 80,100, and $120 \mu \mathrm{m}$ and speed of $100 \mathrm{~mm} / \mathrm{s}$. . 95

4.14 (a) CCT at $110 \mathrm{~V}$ as a function of frequency and (b) uniformity at $110 \mathrm{~V}$ and $400 \mathrm{~Hz}$ for bar coated hybrid EL films using bars with thread thicknesses of $40,60,80,100$, and $120 \mu \mathrm{m}$ and speed of $100 \mathrm{~mm} / \mathrm{s}$. . . . . . . . 95

4.15 (a) EL spectra at $110 \mathrm{~V}$ and $400 \mathrm{~Hz}$ and (b) CIE at $110 \mathrm{~V}$ and 100, 400, 800, 1200,1600, $2000 \mathrm{~Hz}$ for bar coated hybrid EL films using bars with thread thicknesses of $120 \mu \mathrm{m}$ and speed of 10,50 and $100 \mathrm{~mm} / \mathrm{s}$. . . . . 97

4.16 (a) CCT at $110 \mathrm{~V}$ as a function of frequency and (b) uniformity at $110 \mathrm{~V}$ and $400 \mathrm{~Hz}$ for bar coated hybrid EL films using bars with thread thicknesses of $120 \mu \mathrm{m}$ and speed of 10,50 and $100 \mathrm{~mm} / \mathrm{s}$. . . . . . . . . . . 97

4.17 (a) EL spectra at $110 \mathrm{~V}$ and $400 \mathrm{~Hz}$ and (b) CIE at $110 \mathrm{~V}$ and 100, 400, 800, 1200, 1600, $2000 \mathrm{~Hz}$ for hybrid EL films with one layer of bar-coated DCM2 solution at 1.0, 2.0 and $4.0 \mathrm{mg} / \mathrm{mL} \ldots \ldots \ldots$. . . . . . . . 98

4.18 (a) CCT at $110 \mathrm{~V}$ and 100, 400, 800, 1200, 1600, $2000 \mathrm{~Hz}$ and (b) uniformity at $110 \mathrm{~V}$ and $400 \mathrm{~Hz}$ for hybrid EL films with one layer of bar-coated DCM2 solution at 1.0, 2.0 and $4.0 \mathrm{mg} / \mathrm{mL} \ldots \ldots \ldots$. . . . . . . 98 
4.19 (a) EL spectra at $110 \mathrm{~V}$ and $400 \mathrm{~Hz}$ and (b) CIE at $110 \mathrm{~V}$ and 100, 400, $800,1200,1600,2000 \mathrm{~Hz}$ for hybrid EL films with one, two and five layer of bar-coated DCM2 solution at $4.0 \mathrm{mg} / \mathrm{mL} \ldots \ldots . \ldots 99$

4.20 (a) $\mathrm{CCT}$ at $110 \mathrm{~V}$ as a function of frequency and (b) uniformity at $110 \mathrm{~V}$ and $400 \mathrm{~Hz}$ for hybrid EL films with one, two and five layers of bar-coated DCM2 solution at $4.0 \mathrm{mg} / \mathrm{mL}$. . . . . . . . . . . . . . . . . 99

4.21 (a) EL spectra at $110 \mathrm{~V}$ and $400 \mathrm{~Hz}$ and (b) CIE at $110 \mathrm{~V}$ as a function of frequency for hybrid EL films with one layer of bar-coated DCM2fluorescein or DCJTB-fluorescein solution at $15.0 \mathrm{mg} / \mathrm{mL}$ and with the mass ratio of 9:1.

4.22 CCT as a function of voltage at $400 \mathrm{~Hz}$ for hybrid EL films with one layer of bar-coated DCM2-fluorescein or DCJTB-fluorescein solution at $15.0 \mathrm{mg} / \mathrm{mL}$ and with the mass ratio of 9:1. Inset: warm white EL film bar-coated with DCJTB-fluorescein solution operated at $200 \mathrm{~V}$ and $400 \mathrm{~Hz} .101$

4.23 (a) EL spectra and (b) luminance as a function of time for wet-stamped white hybrid EL films with a layer of DCJTB and fluorescein dyes exposed to a constant white light source. . . . . . . . . . . . . . . 102

4.24 (a) EL spectra and (b) luminance as a function of time for wet-stamped white hybrid EL films with a layer of DCJTB and fluorescein dyes operated at $110 \mathrm{~V}$ and $400 \mathrm{~Hz}$. . . . . . . . . . . . . . . . . 102

5.1 EL spectra of (a) forward blue, (b) reversed blue, (c) forward green, and (d) reversed green operated at $400 \mathrm{~Hz}$ and different voltages. . . . . . . . 106

5.2 Normalized EL spectra of (a) forward blue, (b) reversed blue, (c) forward green, and (d) reversed green operated at $400 \mathrm{~Hz}$ and different voltages. . . 107

5.3 EL spectra of (a) forward blue, (b) reversed blue, (c) forward green, and (d) reversed green operated at $110 \mathrm{~V}$ and different frequencies. . . . . . . 108

5.4 (a) Luminance and (b) luminance per cycle as a function of frequency of forward blue, reversed blue, forward green, and reversed green EL films operated at $110 \mathrm{~V}$. . . . . . . . . . . . . . . . . . . 109

5.5 Normalized EL spectra of (a) forward blue, (b) reversed blue, (c) forward green, and (d) reversed green operated at $110 \mathrm{~V}$ and different frequencies. . 110 
5.6 The decomposition of EL spectra operated at $110 \mathrm{~V}$ and $400 \mathrm{~Hz}$ into three emission lines for (a) forward blue, (b) reversed blue, (c) forward green, and (d) reversed green. . . . . . . . . . . . . . . . . 111

5.7 The peak luminance intensities of decomposed emission lines per cycle as a function of frequency for (a) forward blue, (b) reversed blue, (c) forward green, and (d) reversed green. . . . . . . . . . . . . . . . . 112

5.8 Redistribution of injected charge due to drift in an applied field in dependence of frequency according to Fischer's bipolar field emission model. . . 113

5.9 Band diagram for the blue and green emission centers in $\mathrm{ZnS}: \mathrm{Cu}$ phosphors. 115

5.10 Brightness waves of different comet segments at sine wave excitation. Each curve represents the brightness wave of a thirteenth of the comet. The parameter refers to the measured part of the comet: 1 , comet head, 212 , the subsequent parts, 13, comet tail (see lower part of the figure). The curves are slightly smoothed copies of the originally recorded curves, the amplitude being normalized to the maximum of each brightness wave [168] . . 116

5.11 XRD spectra of $\mathrm{ZnS}: \mathrm{Cu}, \mathrm{Cl}$ with $\mathrm{Cu}$ addition in the range of 40 to $5000 \mathrm{ppm}$ fired at $900{ }^{\circ} \mathrm{C}[78] \ldots$. . . . . . . . . . . . . . . . . . 117

5.12 The relation between the kind of luminescence and the concentration of activator $(\mathrm{X})$ and coactivator $(\mathrm{Y}) . \mathrm{X}=\mathrm{Cu}, \mathrm{Ag}, \mathrm{Au}, \mathrm{Y}=\mathrm{Al}, \mathrm{Ga}$, In. "G-Cu": green luminescence in the case of $\mathrm{Cu}$; "B-Cu": blue luminescence in the case of $\mathrm{Cu}$; SA: self-activated luminescence (blue); "R-Cu": red luminescence in the case of $\mathrm{Cu}$; " $\mathrm{R}-\mathrm{Cu}$,In": red luminescence in the case of $\mathrm{Cu}$ and $\mathrm{In}(\mathrm{Ga})[77] . \ldots \ldots 119$

5.13 (a) Configuration in the zinc sulphide lattice caused by incorporation of $\mathrm{CuCl}$ green center [171]; (b) band diagram illustrating donor-acceptor recombination mechanism [174] . . . . . . . . . . . . . . . . . . 120

5.14 (a) Configuration of SA luminescent center in the zinc sulphide cubic cell caused by $\mathrm{C}_{3 \mathrm{~V}}$ structure [178]; (b) band diagram illustrating Schö-Klasens recombination mechanism [179]. . . . . . . . . . . . . . . . . . . 121

5.15 (a) The local environment about $\mathrm{CuS}$ in $\mathrm{ZnS}$ showing the $\mathrm{Cu}$ (red) displaced away from a $\mathrm{S}$ vacancy (light blue) and towards the three remaining $\mathrm{S}$ neighbours (blue) [66]; (b) local atomic arrangement of $\mathrm{B}-\mathrm{Cu}$ luminescent center with $\mathrm{S}$ vacancy and $\mathrm{Cu}_{2} \mathrm{Cl}$ substitutions [171]; (c) Lambe-Klick model for the $\mathrm{B}-\mathrm{Cu}$ recombination mechanism [187] . . . . . . . . . . . 123 
5.16 A charge transfer and relaxation model for the frequency dependent color shift behaviours associated with G-Cu, SA and B-Cu luminescence centers during (a) voltage rise and (b) voltage fall of the positive half cycle. . . . . 126

5.17 G-Cu luminescence intensity as a function of frequency with different charge lifetimes in (a) $\mathrm{Al}$ and (b) $\mathrm{G}-\mathrm{Cu}$ impurity centers. . . . . . . . . . 130

5.18 (a) SA and (b) B-Cu luminescence intensity as a function of frequency with different charge lifetimes in SA and B-Cu impurity centers. . . . . . . . . 131

6.1 Schematic structures of (a) ACPEL devices, (b) a phosphor film, (c) a dielectric film, (d) a binder film with ITO electrodes, and (e) a binder film with one ITO and one Ag electrode used in the temperature dependent impedance spectroscopy study. . . . . . . . . . . . . . . . . 135

6.2 Schematics of the experiment setup for the temperature dependent impedance spectroscopy study of ACPEL devices. . . . . . . . . . . . . 136

6.3 (a) $\varepsilon^{\prime}$ and (b) $\varepsilon$ " as a function of frequency at different temperatures for ACPEL devices. . . . . . . . . . . . . . . . . . . . 138

6.4 (a) $\varepsilon^{\prime}$ and (b) $\varepsilon$ " as a function of frequency at different temperatures for the dielectric film. . . . . . . . . . . . . . . . . 138

6.5 (a) $\varepsilon^{\prime}$ and (b) $\varepsilon$ " as a function of frequency at different temperatures for the phosphor film. . . . . . . . . . . . . . . . . . . 139

6.6 (a) $\varepsilon$ ' and (b) $\varepsilon$ " as a function of frequency at different temperatures for IBI. 139

6.7 (a) $\varepsilon^{\prime}$ and (b) $\varepsilon^{\prime \prime}$ as a function frequency at $90{ }^{\circ} \mathrm{C}$ for ACPEL devices, dielectric film, phosphor film and IBI. . . . . . . . . . . . . . 140

6.8 (a) $M^{\prime}$ and (b) $M^{\prime \prime}$ as a function frequency at different temperatures for the ACPEL devices. . . . . . . . . . . . . . . . . . . . . . 141

6.9 (a) $M^{\prime}$ and (b) $M^{\prime \prime}$ as a function frequency at different temperatures for the dielectric film. . . . . . . . . . . . . . . . . . . . . . 141

6.10 (a) $\mathrm{M}^{\prime}$ and (b) M" as a function frequency at different temperatures for the phosphor film. . . . . . . . . . . . . . . . . . . . . . 142

6.11 (a) M' and (b) M" as a function frequency at different temperatures for IBI. 142

6.12 (a) M' and (b) M"' as a function frequency at different temperatures for IAgBI.143 
6.13 (a) M' and (b) M" as a function frequency at different temperatures for ACPEL devices, dielectric film, phosphor film and IBI. Note: the magnitudes of M' and M" for IBI are scaled by a factor of 0.002 and 0.01 . . . 143

6.14 The decomposition of $M$ " dispersion for (a) ACPEL devices, (b) dielectric

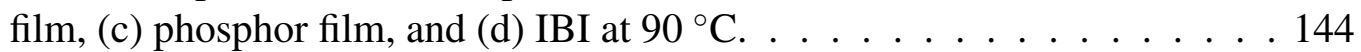

6.15 The dependency of (a) $\alpha$ relaxation and (b) MWS effect for ACPEL devices, dielectric film, phosphor film and IBI at elevated temperatures. Solid lines are simulated results. . . . . . . . . . . . . . . . . 145

6.16 Real conductivity dispersion for (a) ACPEL devices, (b) dielectric film, (c) phosphor film, and (d) IBI at elevated temperatures. . . . . . . . . . . 148

6.17 The dependency of DC conductivities on temperature in ACPEL devices, dielectric film, phosphor film and IBI . Solid lines are simulated results. . . 149

6.18 Experimental and simulated Nyquist plots for (a) ACPEL devices, (b) dielectric film, and (c) phosphor film at $90^{\circ}$. Insets: respective equivalent

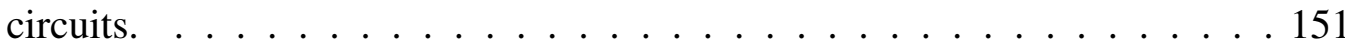

7.1 Experiment setup for opto-impedance measurement and analysis of the ACPEL devices, phosphor and dielectric films. . . . . . . . . . . 155

7.2 (a) $\varepsilon^{\prime}$ and (b) $\varepsilon^{\prime \prime}$ as a function of frequency without illumination and with $399 \mathrm{~nm}, 520 \mathrm{~nm}$ and $625 \mathrm{~nm}$ illuminations for ACPEL devices. . . . . . . 157

7.3 (a) (a) $\varepsilon^{\prime}$ and (b) $\varepsilon$ " as a function of frequency without illumination and with $399 \mathrm{~nm}, 520 \mathrm{~nm}$ and $625 \mathrm{~nm}$ illuminations for the dielectric film. . . 157

7.4 (a) $\varepsilon^{\prime}$ and (b) $\varepsilon$ " as a function of frequency without illumination and with $399 \mathrm{~nm}, 520 \mathrm{~nm}$ and $625 \mathrm{~nm}$ illuminations for the phosphor film. . . . . 158

7.5 (a) $M^{\prime}$ and (b) $M$ " as a function of frequency without illumination and with $399 \mathrm{~nm}, 520 \mathrm{~nm}$ and $625 \mathrm{~nm}$ illuminations for ACPEL devices. . . . . . . 159

7.6 (a) (a) $M^{\prime}$ and (b) $M^{\prime \prime}$ as a function of frequency without illumination and with $399 \mathrm{~nm}, 520 \mathrm{~nm}$ and $625 \mathrm{~nm}$ illuminations for the dielectric film. . . 159

7.7 (a) $M^{\prime}$ and (b) $M$ " as a function of frequency without illumination and with $399 \mathrm{~nm}, 520 \mathrm{~nm}$ and $625 \mathrm{~nm}$ illuminations for the phosphor film. . . . . . 160 
$7.8 \sigma^{\prime}$ as a function of frequency without illumination and with $399 \mathrm{~nm}, 520$ $\mathrm{nm}$ and $625 \mathrm{~nm}$ illuminations for (a) ACPEL devices, (b) dielectric film and (c) phosphor film. . . . . . . . . . . . . . . . . . . . . 161

7.9 Normalized PL emission and excitation spectra of green $\mathrm{ZnS}: \mathrm{Cu}, \mathrm{Al}$ phosphors together with the EL spectra of $399 \mathrm{~nm}, 520 \mathrm{~nm}$ and $625 \mathrm{~nm}$ illuminations. Inset: PL intensity as a function of time after the excitation source is switched off. . . . . . . . . . . . . . . . . . 162

7.10 (a) $\varepsilon^{\prime}$ and (b) $\varepsilon$ " as a function of frequency with the $399 \mathrm{~nm}$ illumination at different intensities for ACPEL devices. . . . . . . . . . . . . . 163

7.11 (a) (a) $\varepsilon^{\prime}$ and (b) $\varepsilon^{\prime \prime}$ as a function of frequency with the $399 \mathrm{~nm}$ illumination at different intensities for the phosphor film. . . . . . . . . . . . 163

7.12 (a) $M^{\prime}$ and (b) $M^{\prime \prime}$ as a function of frequency with the $399 \mathrm{~nm}$ illumination at different intensities for ACPEL devices. . . . . . . . . . . . . . . . 164

7.13 (a) (a) $M^{\prime}$ and (b) $M$ "' as a function of frequency with the $399 \mathrm{~nm}$ illumination at different intensities for the phosphor film. . . . . . . . . . . . 164

$7.14 \sigma^{\prime}$ as a function of frequency with the $399 \mathrm{~nm}$ illumination at different intensities for (a) ACPEL devices and (c) phosphor film. . . . . . . . . 165

7.15 Experimental and simulated Nyquist plots of (a) ACPEL and (b) phosphor films without illumination and with $4.9 \mathrm{~mW} / \mathrm{cm} 2$ illumination together with the corresponding equivalent circuit models. High-energy photons excite the $\mathrm{ZnS}$ phosphor particles. $\mathrm{R}_{\mathrm{s}}$ : series resistance of the electrodes; $\mathrm{R}_{\mathrm{m}}$ and $\mathrm{C}_{\mathrm{m}}$ : resistance and capacitance of the binder matrix; $\mathrm{R}_{\mathrm{ZnS}}$ and $\mathrm{C}_{\mathrm{Zns}}$ : resistance and capacitance of the $\mathrm{ZnS} /$ binder interface; $\mathrm{R}_{\mathrm{BTO}}$ and $\mathrm{C}_{\mathrm{BTO}}$ : resistance and capacitance of the $\mathrm{BaTiO}_{3} /$ binder interface; $\mathrm{R}_{\mathrm{e}}$ and $\mathrm{CPE}_{\mathrm{e}}$ : resistance and constant phase element representing EP; $Z_{\mathrm{w}}$ : Warburg element. 165

7.16 Proposed mechanism for the opto-impedance behaviors of ACPEL devices. (1) Incident photons with energy of higher than $2.95 \mathrm{eV}$ penetrate through ITO/PET and are absorbed by ZnS phosphor particles; (2) electrons are excited from valence band (VB) to conduction band (CB); (a) some electrons trapped in donor sites combine with holes trapped in acceptor sites and emit green phosphorescent lights; (b) some trapped charges drift and are accumulated at the $\mathrm{BaTiO}_{3} /$ binder interfaces; (c) minority charges move to the resin matrix to form dipoles; (d) other charges are trapped at the $\mathrm{ZnS} /$ resin interfaces. . . . . . . . . . . . . . . . . . . . . . 169 


\section{List of Abbrevications}

ACPEL

CCT

CIE

CPE

CRI

DCJTB

DCM2

DUT

EDX

EL

EMI

EP

F8BT

fluorescein

FRA

FWHM

IPA

ITO

LED

LUMO

MWCNT

MWS effect

OLED

PDMS

PEDOT:PSS
AC powder electroluminescence

correlated color temperature

Commission Internationale de l' clairage

constant phase element

color rendering index

4-(Dicyanomethylene)-2-tert-butyl-6-(1,1,7,7tetramethyljulolidin-4-yl-vinyl)-4H-pyran

4-(Dicyanomethylene)-2-methyl-6-julolidyl-9enyl-4H -pyran

device under test

Energy-dispersive X-ray spectroscopy

electroluminescence

electromagnetic interference

electrode polarization

poly[(9,9-dioctylfluorenyl-2,7-diyl)-alt(benzo[2,1,3]thiadiazol-4,7-diyl)]

Sodium 2-(3-hydroxy-6-oxido-9H-xanthen-9yl)benzoate

frequency response analyzer

full width at half maximum

isopropanol

indium tin oxide

light emitting diode

lowest unoccupied molecular orbital

multiwall carbon nanotube

Maxwell-Wagner-Sillars effect

organic light emitting diode

polydimethylsiloxane

poly(3,4-ethylenedioxythiophene) polystyrene sulfonate 
PET

PL

PVDF

RPM

SEM

SWCNT polyethylene terephthalate

photoluminescence

polyvinylidenedi fluoride

revolutions per minute

scanning electron microscope

single wall carbon nanotube 


\section{Chapter 1}

\section{INTRODUCTION}

\subsection{Problem statement and motivation}

Printed flexible and stretchable electronic devices have attracted a great deal of research interest in recent years because these functional devices are low cost, disposable and well suited to mobile and wearable applications [1]. Although many devices, such as field effect transistors, sensors, solar cells, batteries and supercapacitors have been demonstrated [2], the printed sheet lighting source is less well developed. A low-cost printed white light film with high luminance, efficacy, large emission area, light weight and adequate color temperature and lifetime will have many applications such as signage, advertising and solid-state lighting. One device structure is the organic light emitting diode (OLED) based on multiple evaporated layers of phosphorescent emitting layers and charge transport/blocking layers [3]. Although high performance white OLEDs have been reported [4], the costs of both organic semiconductor materials and the batch fabricating process remain high $[5,6]$. Furthermore, organic materials are sensitive to moisture and oxygen and must be encapsulated by passivation films which add to the cost [7]. As a result, a lot of research efforts are shifted backward to conventional inorganic LED due to its lower cost and mature process, especially silicon based LEDs [8-12].

Despite its high energy saving capability, bright and white LED light sources when used in outdoor conditions are the main contributor to the global light pollution, which is increasing rapidly at around $6 \%$ per annum $[13,14]$. One of the concerns in global light pollution is the ecological consequences caused by LED light sources. It has been found that the intrusion of artificial light has profound effects on wildlife including plants, invertebrates, fish, amphibians, reptiles, birds and mammals [15-22]. In another aspect, the 
indoor LEDs are evident to cause issues on the human circadian clock [23], photoreceptor loss [24], and visual perception [25]. The problem of LED lies in the strong blue content of the white light which is dominated by a narrow, short-wavelength emission band together with a broader long-wavelength emission band. In human's eyes, there is one type of photoreceptor cell called S-cones that are most sensitive to blue or blue-violet lights [26]. The strong blue emission from LEDs has been observed to be the main cause of human health issues [27, 28]. Therefore, warm white LEDs with low CCT are highly favored [29, 30]. However, in a recent study by Pawson and Bader [31], reducing the CCT of white LEDs does not improve the ecological performances due to the persistent presence of the blue emissions.

An alternative sheet lighting source is the much earlier ACPEL device based on activated and encapsulated $\mathrm{ZnS}$ phosphor powders. The main advantage of ACPEL devices is its low cost due to the simple printing process, uniform sheet light emission and low power consumption [32]. However, conventional ACPEL devices still suffer from low luminance, low efficacy and a limited choice of colors [33-35]. ZnS phosphors emitting at longer wavelengths have been reported [36,37]. However, their performance is even poorer than the standard blue and blue/green phosphors. This limitation is especially problematic for the realization of a warm white lighting source. Therefore, the hybrid ACPEL device structure is necessary for the realization of warm white light source.

\subsection{Scope and objectives}

The primary objective of this study is to fabricate a warm white light source based on the ACPEL device structure hybrid with downshifting materials and to understand the operational characteristics of the hybrid EL films. Therefore, the scope of this study includes:

1. To develop and optimize low cost processes for the printing of hybrid device structures. 
2. To select downshifting materials and formulate printing agents for the fabrication of warm white EL films.

3. To characterize the hybrid devices at varied operating conditions and obtain the luminous efficacy.

4. To build a physics based model to understand the operating characteristics.

\subsection{Significance of the research}

This research work has produced significant outcomes which include:

1. Fabricated hybrid white/warm-white EL films with the combination of modified processes including screen printing, drop casting, wet stamping and bar coating.

2. Fabricated warm white lighting source with the lowest CCT of $2820 \mathrm{~K}$ and highest luminance of $72.28 \mathrm{~cd} / \mathrm{m}^{2}$.

3. Establishment of a charge transport and relaxation model to explain the frequency dependent color shift behavior and low luminous efficacy from $\mathrm{ZnS}$ level.

4. Fundamental understanding of the device level physics of operated EL film and identification of device level root cause of low luminous efficacy.

\subsection{Organization of thesis}

This thesis consists of 8 chapters, including introduction (Chapter 1), literature review (chapter 2), research findings (Chapter 3 to 7) and conclusions and future works (Chapter 8).

Chapter 1 gives an introduction of the current status of solid state lighting industry and the need for a warm white sheet lighting source. Chapter 2 thoroughly reviews the fundamentals of ACPEL devices, various printing and coating techniques and dielectric impedance spectroscopy technique, which act as a foundation for this research work. 
Chapter 3 presents the experimental procedures together with solution formulation for drop casting of poly[(9,9-dioctylfluorenyl-2,7-diyl)-alt-(benzo[2,1,3]thiadiazol-4,7-diyl)] (F8BT) and 4-(Dicyanomethylene)-2-tert-butyl-6-(1,1,7,7-tetramethyljulolidin-4-yl-vinyl)4H-pyran (DCJTB) dyes on forward and reversed blue and green EL films. The downshifting capability of F8BT and DCJTB was explored. The optical properties of conventional EL films and hybrid films are also presented. The effects of concentration and mass ratio on the color output of hybrid films are discussed.

Chapter 4 demonstrates the feasibility of using a modified wet stamping process and bar coating technique for the fabrication of white and warm white hybrid EL films. The experimental conditions for these two methods were optimized and different combinations of DCJTB, 4-(Dicyanomethylene)-2-methyl-6-julolidyl-9-enyl-4H -pyran (DCM2) and Sodium 2-(3-hydroxy-6-oxido-9H-xanthen-9-yl)benzoate (fluorescein) dyes were tested.

In Chapter 5, the frequency dependent color shift in green and blue EL films was studied experimentally and the resulted spectra were analyzed. The excitation mechanisms, luminescent centers and recombination mechanisms of green and blue emissions were reviewed and a charge transport and relaxation model was proposed to explain the observed phenomenon.

Chapter 6 and 7 present a new opto-impedance spectroscopy apparatus to characterize the frequency-dependent impedance of EL films. The temperature dependent dielectric properties were obtained and analyzed to determine relaxation processes present within the films. The effects of illumination wavelengths and intensities were discussed and a physics based equivalent circuit model was constructed and verified to represent the relaxation processes. The charge distribution during excitation was illustrated to understand the low luminous efficacy of hybrid films. 


\section{Chapter 2}

\section{LITERATURE REVIEW}

This chapter reviews the fundamentals of ACPEL phenomenon, device structures, its light emission mechanisms and the constructing materials. The printing and coating techniques are also covered. In particular, the dielectric impedance spectroscopy technique is reviewed in details.

\subsection{ACPEL devices}

\subsubsection{History of ACPEL devices}

ACPEL phenomenon was first discovered by Round in 1907 who was working on silicon carbide [38], and was later observed by Lossew [39]. However, it was until 1936 that this field became actively researched when Destriau worked on copper-doped $\mathrm{ZnS}$ [40]. Since then, enormous researchers had worked on understanding the operating characteristics, excitation and degradation mechanisms of ACPEL devices [41-53]. However, the EL panels made of luminescent powders suffered from low luminance and short half-life of less than 500 hours. As a result, attention was shifted towards DC powder EL and thin film EL devices. During the period from the late 1970s to the 1980s, there was a need to develop flat panels instead of cathode-ray tube displays driven by the increasing wide applications of computer technology. In 1974, high luminance and lifetime as long as 10,000 hours were achieved in AC-driven, high field, thin-film EL devices [54].

A second push on the ACPEL devices occurred after the development of more durable phosphors around 1990s. Steady improvement in both the luminance and lifetime/reliability of phosphor powders was achieved by coating a protective layer over the phosphor powders. Due to this advancement, ACPEL devices were extensively used in back-lighting of 
liquid crystal displays (LCDs), mobile phone keyboards, automotive dashboards, indoor illumination at modest light levels, safety installations and lighting for advertising purposes or decoration until today [34]. After that, most of the research efforts were devoted to develop LEDs, especially OLEDs. In recent years, ACPEL phenomenon was revisited due to the potential and demand of flexible displays and large area lighting applications and the need to reduce the production cost of today's lighting industry.

\subsubsection{Device architectures}

A Destriau-type ACPEL cell consists of phosphor powders suspended in a castor oil. This suspension is sandwiched between two electrodes of which at least one electrode is transparent. This architecture is still widely used in laboratory for quick demonstration of the electroluminescence of newly made phosphor powders. Meanwhile, commercially, there are four types of architectures: forward, reversed, interdigitated and combined ACPEL devices.

\section{Forward architecture}

As a conventional structure, the forward ACPEL devices are the most used in commercial products as shown in Figure 2.1. A phosphor layer and a dielectric layer are sandwiched between front and back electrodes. This dielectric layer is to prevent the electric breakdown and protect the phosphors from moisture. For forward ACPEL devices, light is emitted from the substrate side. Therefore, the front electrode and substrate have to be as transparent as possible.

\section{Reversed architecture}

The reversed ACPEL devices, apparently, have the reversed structure compared with the forward ACPEL devices. As illustrated in Figure 2.2, the back electrode is directly attached to the substrate while the dielectric and phosphor layers are in a reversed order. In 


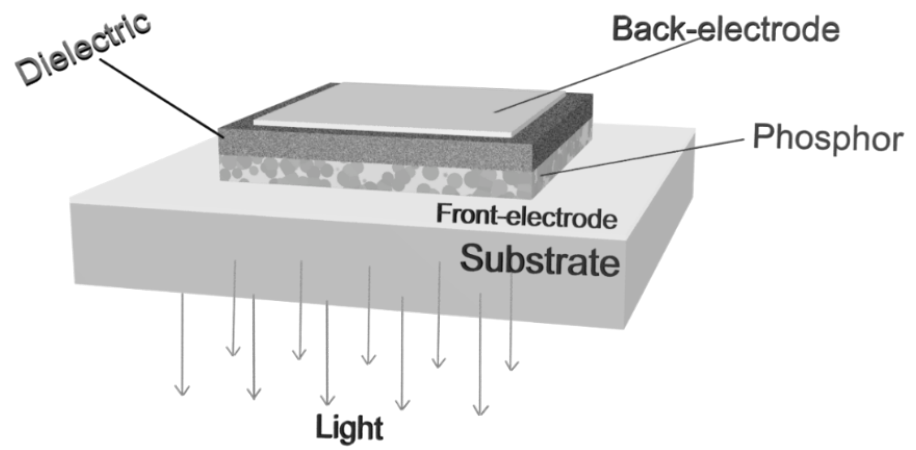

Figure 2.1: Architecture of forward ACPEL devices. Light is emitted from the substrate side.

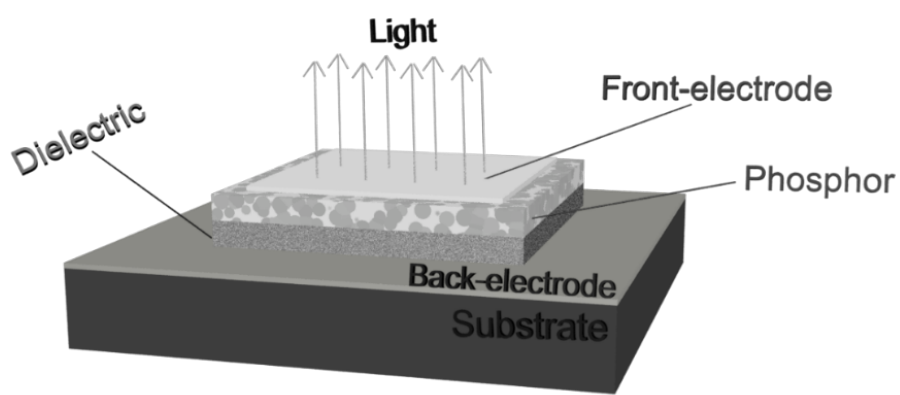

Figure 2.2: Architecture of reversed ACPEL devices. Light is emitted from the front electrode.

reversed ACPEL devices, light is not emitted from the substrate but from the front electrode. Therefore, only the front electrode needs to be transparent. This architecture has higher reliability as the dielectric layer is more uniformly printed than the forward devices but with lower luminance.

\section{Interdigitated architecture}

Another innovative approach to fabricate ACPEL devices is the interdigitated architecture as shown in Figure 2.3. This structure does not require any transparent electrode. Instead, electrodes are printed in an interdigitated pattern onto the substrate using lithographic printing [55]. Only a phosphor layer is present without any dielectric layer required. The electrode gaps need to be narrow and are normally between 100 and $200 \mu \mathrm{m}$. Due to limited light emitting sites, this architecture suffers from low luminance. 


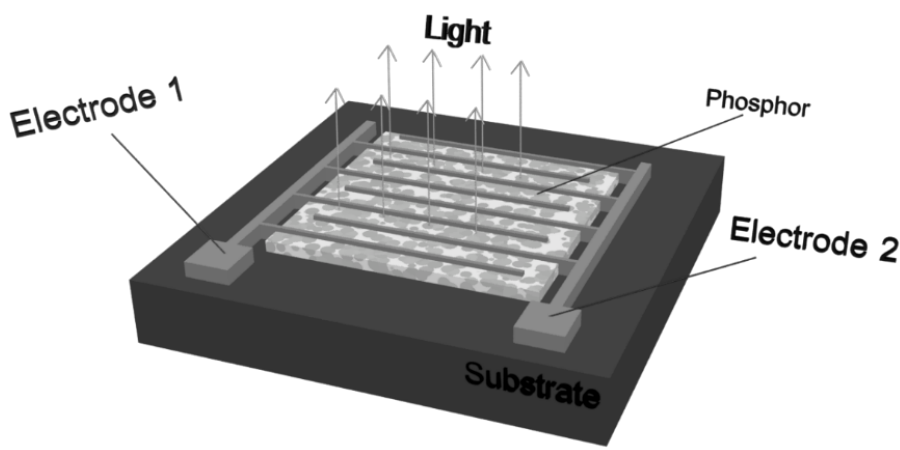

Figure 2.3: Architecture of interdigitated ACPEL devices. Light is emitted from phosphors that are within the two parallel electrodes.

\section{Combined architecture}

The combined architecture combines the phosphor and dielectric layers into one single layer as shown in Figure 2.4. The other layers are the same as the conventional forward architecture. As no additional dielectric layer is present, the amount of dielectric material in the combined layer needs to be properly defined. Too few dielectric materials will cause high chance of early failure resulted by dielectric breakdown. Too many dielectric materials will reduce the light output intensity. More research efforts are needed to balance these two factors and currently no commercial products are based on this architecture. However, the attractive point of this architecture is that it is more suitable for use in flexible displays. This is because less amount of layers will introduce less internal stress when the device is subjected to bending or stretching. If the back electrode is made transparent, this device is then luminescent from both side. With the proper choice of dielectric material, transparent display devices may be feasible.

\subsubsection{Materials for forward and reversed ACPEL devices}

Materials for the individual layers of forward and reversed ACPEL devices are listed in Table 1. There are mainly four categories of materials for the four layers of ACPEL devices, namely substrate, electrodes, phosphor, and dielectric layers. The advantages and disad- 


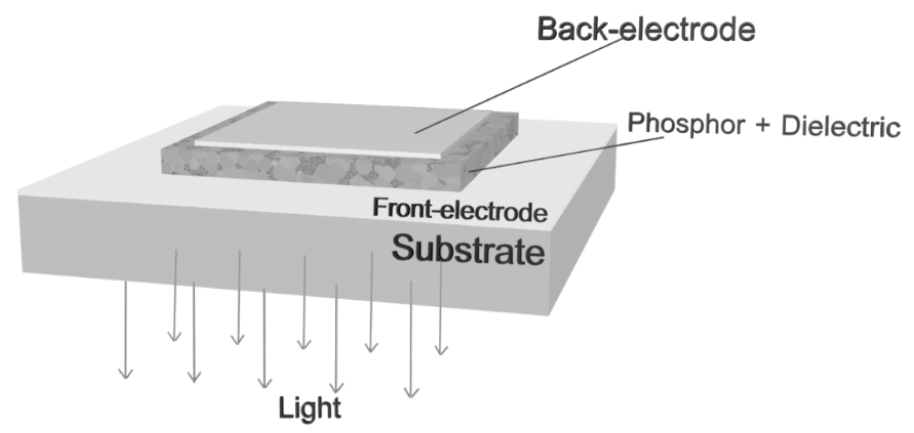

Figure 2.4: Architecture of combined ACPEL devices. Light is emitted from the substrates side.

vantages of choosing the material for the specific layer are listed in Table 2.1. Following are the detailed reviews of the materials used for ACPEL devices.

\section{Substrate}

The main material for the substrate of forward ACPEL devices is polyethylene terephthalate (PET) or equivalent transparent polymers. It is a thermoplastic polymer and is very resistive to water and moisture. Depending on the process, PET can be totally transparent or semi-transparent. The production cost for PET is low and its flexibility makes it suitable for flexible display applications. However, due to its poor thermal resistivity, PET-made ACPEL devices tend to warp at high temperatures. As a polymer, PET is also subjected to polymer degradation in a long run. Another alternative transparent substrate is glass. It is relatively cheap and also has low water permeability. An advantage for glass is that it has higher thermal resistivity compared to PET which makes it a better choice for high temperature applications. As for reversed ACPEL devices, theoretically it can be printed over any substrate as long as it is flat and adhesive enough to the printed back electrodes.

\section{Electrode}

ACPEL devices have two types of electrodes: front and back electrodes. The front electrode needs to be as transparent as possible for higher light output. The back electrode is not 
Table 2.1: Pros and Cons for the materials used in each layer of the ACPEL devices.

\begin{tabular}{|c|c|c|c|}
\hline Layer & Materials & Pros & Cons \\
\hline \multirow[t]{2}{*}{ Substrate } & PET & $\begin{array}{l}\text { Good moisture resistivity; } \\
\text { Flexible; Low cost; Easy for } \\
\text { machining }\end{array}$ & $\begin{array}{l}\text { Poor thermal resistivity; } \\
\text { Polymer degradation }\end{array}$ \\
\hline & Glass & $\begin{array}{l}\text { Good moisture resistivity; } \\
\text { high thermal resistivity; low } \\
\text { cost }\end{array}$ & $\begin{array}{l}\text { Not suitable for flexible } \\
\text { display; hard for machining }\end{array}$ \\
\hline \multirow{2}{*}{$\begin{array}{l}\text { Back } \\
\text { electrode }\end{array}$} & Metal & Highly conductive & Expensive \\
\hline & Carbon & Low conductivity & Cheap \\
\hline \multirow[t]{2}{*}{$\begin{array}{l}\text { Front } \\
\text { electrode }\end{array}$} & ITO & $\begin{array}{l}\text { High conductivity; } \\
\text { transparent }\end{array}$ & $\begin{array}{l}\text { Expensive; limited source } \\
\text { (Indium); cracking issue } \\
\text { under stresses }\end{array}$ \\
\hline & $\begin{array}{l}\text { PE- } \\
\text { DOT:PSS }\end{array}$ & $\begin{array}{l}\text { Cheap; possible for flexible } \\
\text { displays }\end{array}$ & $\begin{array}{l}\text { Low conductivity; } \\
\text { semi-transparent; } \\
\text { transmission loss }\end{array}$ \\
\hline \multirow[t]{2}{*}{ Phosphor } & $\mathrm{ZnS}$ & $\begin{array}{l}\text { Mature fabricating process; } \\
\text { chemically stable }\end{array}$ & $\begin{array}{l}\text { Limited color choice; short } \\
\text { lifetime }\end{array}$ \\
\hline & $\begin{array}{l}\text { Alkaline } \\
\text { earth } \\
\text { sulfides }\end{array}$ & More color choice & $\begin{array}{l}\text { Chemically unstable; shorter } \\
\text { lifetime }\end{array}$ \\
\hline Dielectric & $\mathrm{BaTiO}_{3}$ & High dielectric constant & $\begin{array}{l}\text { Phase transition at high } \\
\text { temperature }\end{array}$ \\
\hline
\end{tabular}

necessary to be transparent but should have high electrical conductivity.

Metal powders or carbon black are suitable to be the back electrode. Metal powders such as $\mathrm{Ag}$ or $\mathrm{Au}$ are highly conductive but expensive. Others think it may fail due to electromigration [34]. However, for such low current (10 to $20 \mathrm{~mA}$ ), it is hard for electromigration to occur. Another cheaper choice is carbon but with limited applications due to its low conductivity. High conductivity is crucial for ACPEL devices because the homogeneity of the electrical field over the active layer is affected by the conductivity of the 
back electrode. Low conductivity will produce inhomogeneous distribution of emitted light intensity. For small area devices, carbon back electrode is sufficient. However, for large panels, it requires highly conductive metal electrodes.

The choices for the transparent front electrode are more constrained compared to that for the back electrode. Indium tin oxide (ITO) is widely adopted due to its relatively high electrical conductivity but is quite expensive and uses a limited source indium. An alternative electrode are conductive polymers like poly(3,4-ethylenedioxythiophene) polystyrene sulfonate (PEDOT:PSS). It is mostly used in flexible display as ITO has cracking issues under large stresses. However, its limited conductivity (commercially $200 \Omega /$ sq compared to $10-100 \Omega / s q$ for ITO) makes PEDOT:PSS only suitable for moderate-size panels. One disadvantage for PEDOT:PSS is that it is light grey in color and not as transparent as ITO. Moreover, PEDOT:PSS experiences color shift with time and causes transmission loss to ACPEL devices. As a polymer, PEDOT:PSS is also susceptible to thermal and humidity degradations [56-58]. Nevertheless, for the reversed ACPEL devices, PEDOT:PSS is more suitable as the front electrode as it is printable while ITO requires vacuum deposition process.

\section{Phosphors}

Commercial ACPEL devices are dominated by ZnS-phosphors. EL ZnS phosphor powders are prepared by firing at high temperature $\left(1100-1200{ }^{\circ} \mathrm{C}\right)$ and being cooled to form cubic zinc-blende structure [47]. Intrinsic $\mathrm{ZnS}$ is not able to produce light under electrical excitation and dopants such $\mathrm{Cu}, \mathrm{Ag}, \mathrm{Au}, \mathrm{Mn}$ (acceptors) and $\mathrm{Cl}, \mathrm{Br}, \mathrm{Al}$ (donors) are needed. Moreover, only donors and acceptors with levels close enough to the band edges of $\mathrm{ZnS}$ are entitled as luminescent centers. Detailed review of the excitation mechanism will be discussed in the next section. The emission colors can be controlled by adding different dopants but the choice of colors is limited $(\mathrm{ZnS}: \mathrm{Cu}, \mathrm{Cl}$ emitting at $450 \mathrm{~nm}, \mathrm{ZnS}: \mathrm{Cu}, \mathrm{Al}$ at $540 \mathrm{~nm}, \mathrm{ZnS}: \mathrm{Cu}, \mathrm{Mn}, \mathrm{Al}$ at $590 \mathrm{~nm}$ and $\mathrm{ZnS}: \mathrm{Cu}$ at $690 \mathrm{~nm}$ ). The solubility of $\mathrm{Cu}$ in $\mathrm{ZnS}$ 
is very low (only $800 \mathrm{ppm}$ ) [59]. For large area lighting applications, it is still a challenge to generate stable white emission. Generally, white light emission is achieved by mixing orange and blue emitting phosphors or combining a blue or green emitter with a fluorescent dye generating the necessary longer wavelengths [60]. Besides the poor color rendering problem, the most severe problem is that the different maintenances (i.e. degradation behaviors) of different phosphors in the blends lead to the change of emitted light color gradually as the devices degrade.

Other than $\mathrm{ZnS}$, experimentally demonstrated capable ACPEL phosphors are all based on alkaline earth sulfides, namely $\mathrm{CaS}, \mathrm{BaS}$ or $\mathrm{SrS}$. Similar to $\mathrm{ZnS}$, most of these alkaline earth sulfides need to be coupled with small concentration of $\mathrm{Cu}$ to produce light. However, more combinations of different donors are reported regarding the emission from lanthanide ions, e.g., $\mathrm{CaS}: \mathrm{Cu}, \mathrm{Eu}$ [61], $\mathrm{CaS}: \mathrm{Cu}, \mathrm{Sm}$ [62], $\mathrm{CaS}: \mathrm{Cu}, \mathrm{Nd}$ [63], $\mathrm{CaS}: \mathrm{Cu}, \mathrm{Er}$ [64]. There are also papers showing EL emissions without $\mathrm{Cu}$ dopants in $\mathrm{CaS}$ :Eu [65]. Similar EL phosphors can be fabricated for $\mathrm{SrS}, \mathrm{BaS}$ and $(\mathrm{Sr}, \mathrm{Ba}) \mathrm{S}$. However, all these materials are much more reactive with $\mathrm{H}_{2} \mathrm{O}$ than $\mathrm{ZnS}$ and thus degrade much faster. Therefore, none of these phosphors are used commercially for the fabrication of ACPEL devices.

Another possible phosphor is the $\mathrm{ZnS}$ nanoparticle in the size of 1-10 nm which has been demonstrated in the laboratory but not commercially [66-69]. Although various attempts have been tried to build ACPEL devices based on $\mathrm{ZnS}$ nanoparticles, their performances are not up to satisfactory with low stability and maintenance [70-72]. Further developments are needed to solve the packaging and stabilization issues.

\section{Dielectrics}

The dielectric layer has two functions. One function is to prevent electrical breakdown and the other is to reflect the emitted light and also insulate phosphors from moistures. The most used dielectric material in ACPEL devices is $\mathrm{BaTiO}_{3}$. It has high dielectric constant with up to 2000-3000 at room temperature and up to 10,000 at $400 \mathrm{~K}$ (the curie tempera- 
ture of the ferroelectric phase) [34]. However, the dielectric properties of $\mathrm{BaTiO}_{3}$ powders are dependent on the preparation procedures, processing conditions and final morphology. Normally an elegant solution mixing $\mathrm{BaTiO}_{3}$ nanoparticles with binders is made for the printing process. The binder is polymer materials and has relatively high dielectric constant. Polyvinylidenedi fluoride (PVDF) is commonly used as the binder material.

\subsubsection{Properties of $\mathrm{ZnS}$ and its phosphor powders}

\section{Microstructure}

$\mathrm{ZnS}$ is a IIb-VIb compound direct bandgap semiconductor. ZnS has two common crystal structures: a cubic zinc blende (sphalerite) structure and a hexagonal (wurtzite) structure. The bandgap of the sphalerite form of $\mathrm{ZnS}$ is $3.72 \mathrm{eV}$ while that of the wurtzite structure is $3.77 \mathrm{eV}$ [73-75]. The phase diagram of a Zn-S system is shown in Figure 2.5. At temperatures below $1000{ }^{\circ} \mathrm{C}, \mathrm{ZnS}$ has a sphalerite structure $(\alpha \mathrm{ZnS})$ regardless of the sulphur percentage. On the contrary, the wurtzite structure $(\beta \mathrm{ZnS})$ is only formed at temperature above $1000{ }^{\circ} \mathrm{C}$ and with sulphide percentage of $50 \%$.

ZnS without any dopant only has UV emission but no visible light emissions [77]. Moreover, $\mathrm{ZnS}$ exhibits EL phenomenon only when the concentration of $\mathrm{Cu}$ dopant exceeds $800 \mathrm{ppm}$ [59]. In Figure 2.6, the XRD spectra of the ZnS phosphor with concentrations of $\mathrm{Cu}$ ranging from 40 to $5000 \mathrm{ppm}$ show that hexagonal and cubic phases co-exist with $\mathrm{Cu}$ concentration below 800 ppm. When $\mathrm{Cu}$ concentration exceeds $800 \mathrm{ppm}$, the hexagonal phase disappears. Therefore, there is phase transition occurring at $800 \mathrm{ppm}$.

As observed by Fischer using an optical microscope [45], there are birefringent lines extending parallel over the $\mathrm{ZnS}$ phosphor particles. When the $\mathrm{ZnS}$ is excited by an $\mathrm{AC}$ electric field, these lines become luminescent and exhibit a "double comet" shape as shown in Figure 2.7. Fischer ascribed it to be $\mathrm{Cu}_{2} \mathrm{~S}$ precipitates which was also called needles. Based on these observations, the dipolar field emission theory was proposed, which will be covered in the next section. However, other scientists do not observe these needles. 


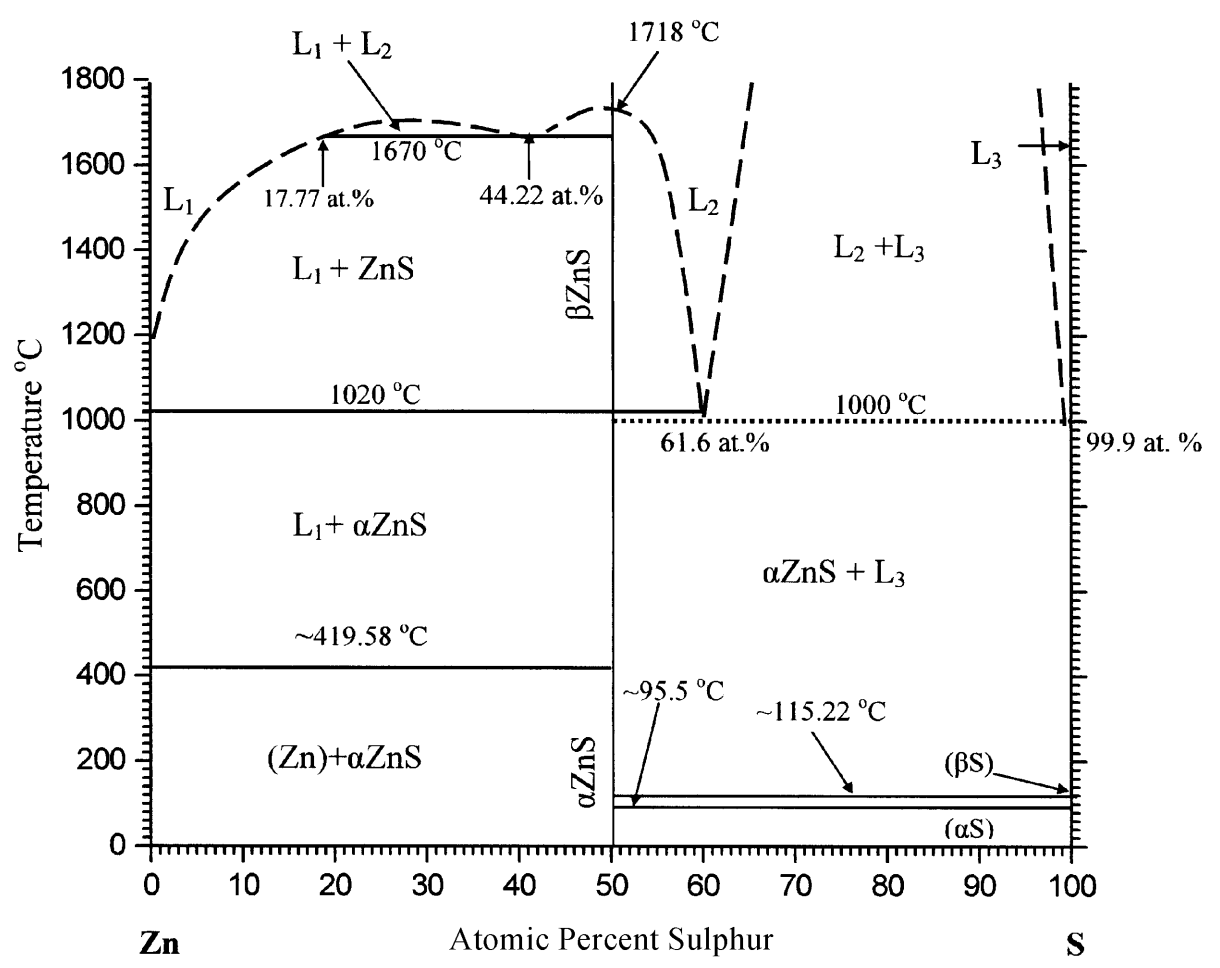

Figure 2.5: Binary phase diagram for the $\mathrm{Zn}-\mathrm{S}$ system [76].

Instead, when $\mathrm{ZnS}$ phosphor powders were etched with hydrochloric acid, a regular layered structure was observed as shown in Figure 2.8(a) [79]. A closer look at the layered structure by TEM shows twin microstructure with a narrow intergrowth wurtzite type phase as shown in Figure 2.8(b) [80]. Based on these observations, the junction model was proposed by Brovetto et al. [81].

\section{Point defects in $\mathrm{ZnS}$}

The nature and concentration of defects are important as they are crucial in determining the optical and electrical properties of semiconductors. There are intrinsic and extrinsic point defects in $\mathrm{ZnS}$. As a binary compound, intrinsic point defects in $\mathrm{ZnS}$ are in the form of vacancies and interstitials on or in both the cation $\left(\mathrm{Zn}^{2+}\right)$ and anion $\left(\mathrm{S}^{2-}\right)$ sublattices. However, the types and equilibrium defect concentrations of intrinsic defects are largely dependent on the deposition technique, deposition conditions and substrate materials. Assignment of energy levels in the band gap to specific structural defects is often nontrivial [82], and the 


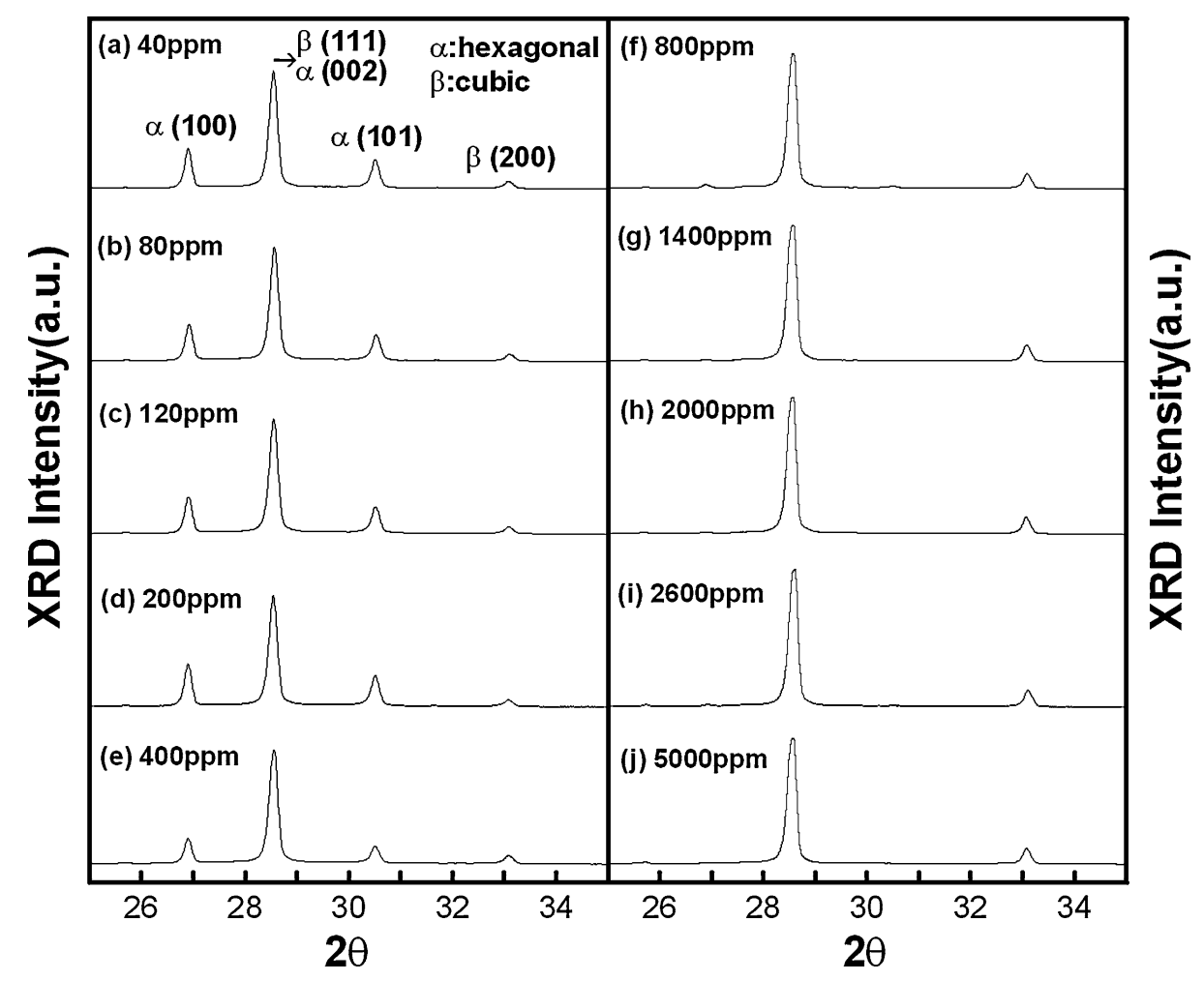

Figure 2.6: $\mathrm{XRD}$ spectra of $\mathrm{ZnS}: \mathrm{Cu}, \mathrm{Cl}$ with $\mathrm{Cu}$ addition in the range of 40 to $5000 \mathrm{ppm}$ fired at $900{ }^{\circ} \mathrm{C}[78]$.

electronic properties of point defects in compound semiconductors are not fully understood because of their variety [83].

The most well characterized doubly ionized $\mathrm{Zn} /$ cation vacancy $\left(\mathrm{V}^{2+} \mathrm{Zn}\right)$ is reported to have a defect level $\sim 1.0-1.1 \mathrm{eV}$ above the $\mathrm{ZnS}$ valence band edge [84-86]. This value is close to the depth of hole trap at $1.2 \mathrm{eV}$ reported by Hitt et al. [87]. The defect level of a singly ionized $\mathrm{V}^{+} \mathrm{Zn}$ vacancy is reported to be in the range of $0.2-0.6 \mathrm{eV}$ above the valence band $[85,86]$. The electrical characteristics of anion vacancies in II-VI compounds are less well known [88]. As shown in Figure 2.9, the donor levels of a chlorine-doped $\mathrm{ZnS}$ are at $0.515,0.615$ and $1.08 \mathrm{eV}$ below the conduction-band edge as revealed by the impurity absorption and photoconductivity measurements [85].

The extrinsic point defects in $\mathrm{ZnS}$ are originated from the presence of impurities or intentional dopants in the lattice. The significance of the extrinsic defects lies in the creation of $\mathrm{n}$ and $\mathrm{p}$ type semiconductors, enabling the subsequent radiative recombination or 


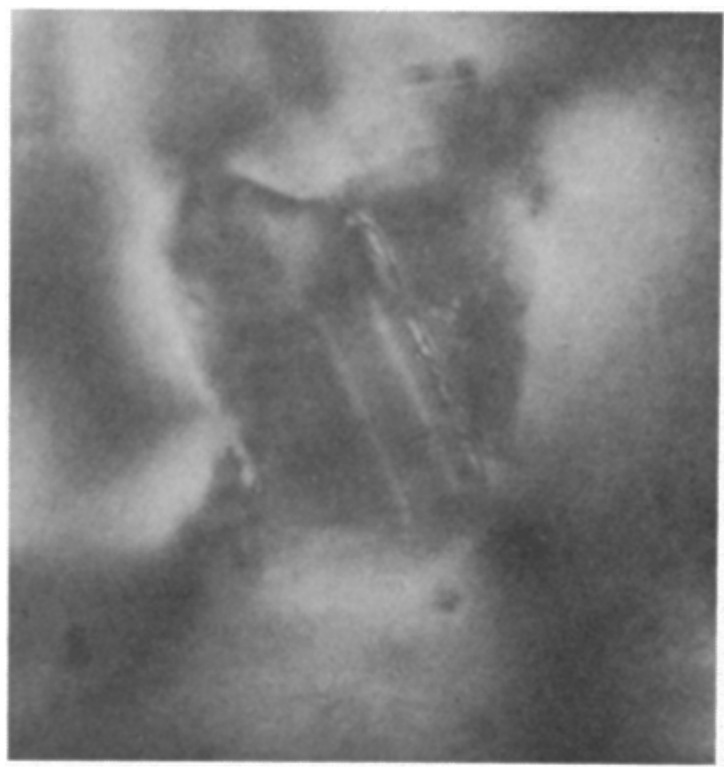

(a)

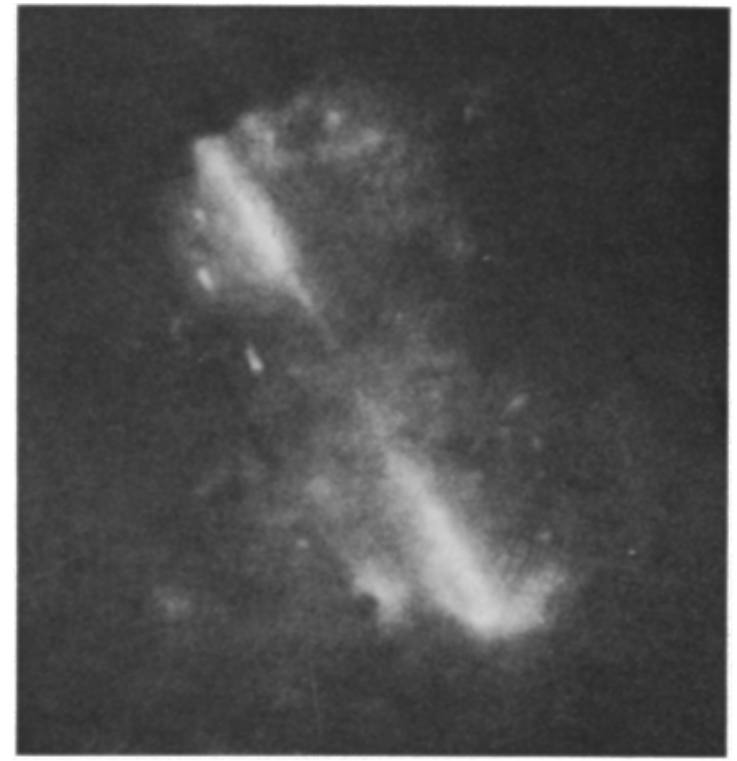

(b)

Figure 2.7: Observation of Electroluminescent lines in ZnS Powder Particles. (a) birefringent lines extending parallel to long cavity. Bright area when the device is not excited. (c) $\mathrm{ZnS}$ is excited by an $\mathrm{AC}$ electric field . Electroluminescent double line at site of the birefringent striation [45].

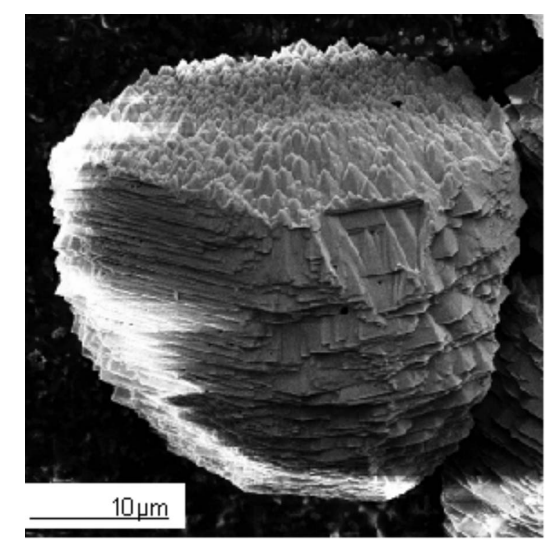

(a)

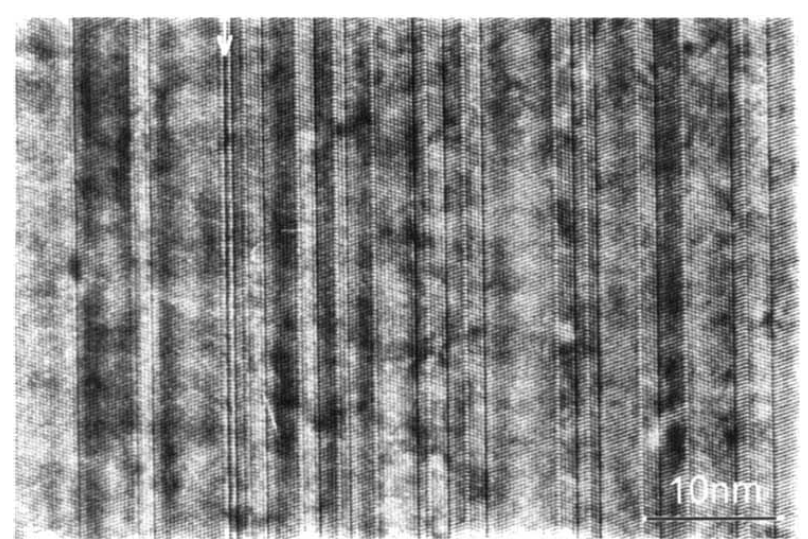

(b)

Figure 2.8: (a) SEM image of a $\mathrm{ZnS}$ :Cu-based particle after etching with hydrochloric acid for $15 \mathrm{~min}$, showing a layered structure [79]. (b) Cross-sectional TEM images of a $\mathrm{ZnS}$ phosphor grain. Twin microstructure is seen at high resolution, together with a narrow intergrowth of wurtzite type indicated by the white arrow [80].

injection type electroluminescence [89]. However, no p-type $\mathrm{ZnS}$ has been demonstrated to date and consequently the energy levels regarding the acceptor defects in $\mathrm{ZnS}$ have little 


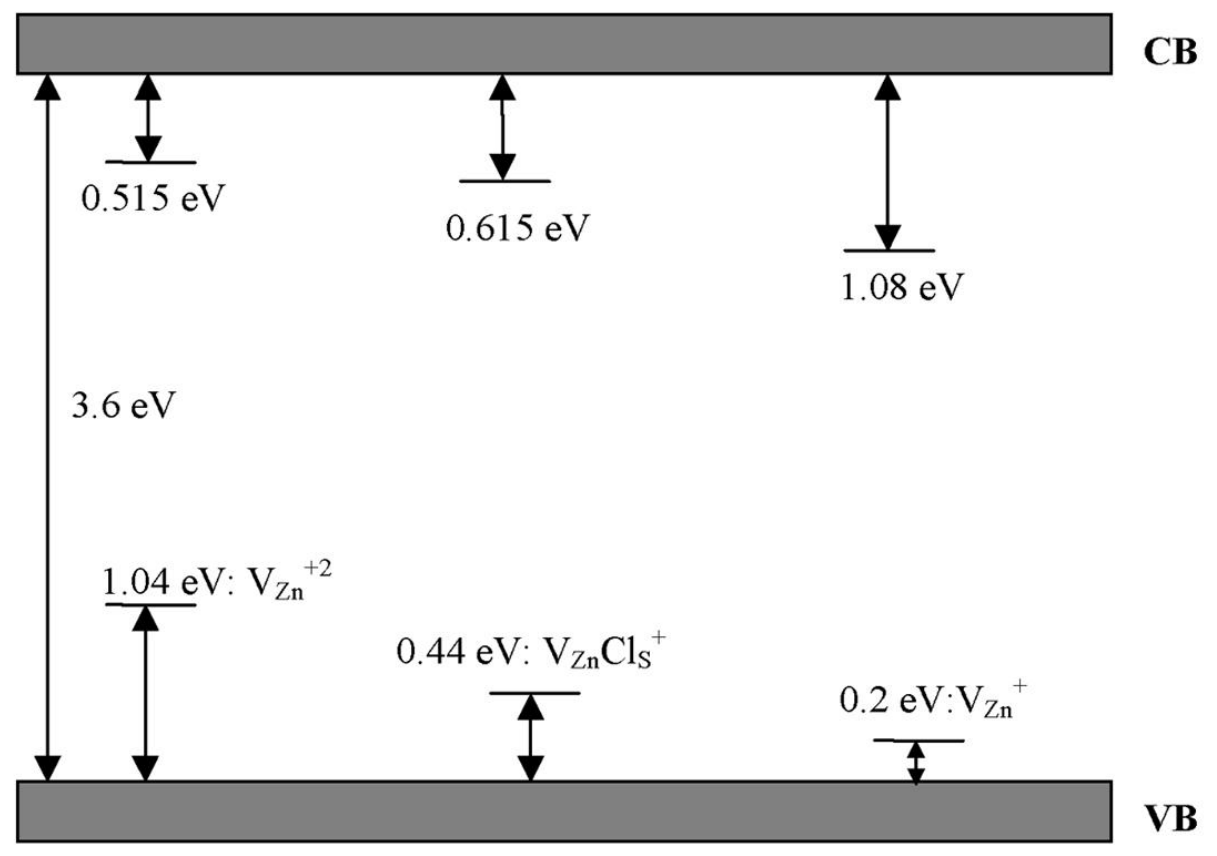

Figure 2.9: Schematic band-gap diagram of $\mathrm{ZnS}: \mathrm{Cl}$ showing deep defect levels [85].

been reported $[90,91]$. Many of the rare-earth ions in their $2+/ 3+$ oxidation state are employed to form luminescent centers in $\mathrm{ZnS}$ by substituting on to tetrahedrally coordinated $\mathrm{Zn}$ lattice sites. The detailed review of the luminescence centers in $\mathrm{Cu}$ doped $\mathrm{ZnS}$ will be presented in the next section.

\section{Emission centers in Cu doped $\mathrm{ZnS}$}

$\mathrm{Cu}$ doped $\mathrm{ZnS}$ is so far the most stable and efficient phosphor powder used in commercial ACPEL devices. However, the exact nature of $\mathrm{ZnS}: \mathrm{Cu}$ phosphor emission centers is poorly understood. Pure $\mathrm{ZnS}$ has a UV emission but no visible luminescence. However, when S is substituted by $\mathrm{Cl}^{-}$, a blue line is observed. This material is referred to as self-activated (SA). The blue light is probably due to the movement of an electron trapped on a $\mathrm{Cl}^{-}$site to a $\mathrm{Zn}$ vacancy aggregates [77]. When only $\mathrm{Cu}$ dopants are added, there are two emission lines: a blue line very close to that for the SA center and a red line. Bowers and Melamed did detailed susceptibility measurements and showed that copper is in $\mathrm{Cu}^{+}$state in all these samples without any excitation, including samples with higher $\mathrm{Cu}$ concentrations [41]. 
CB

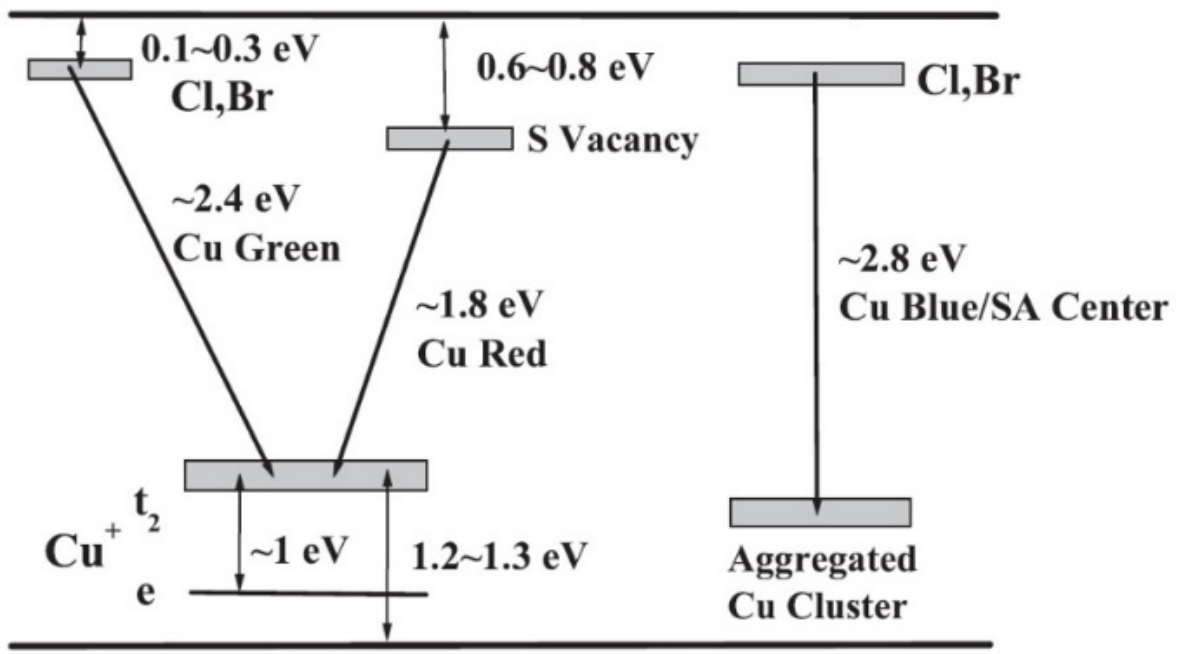

VB

Figure 2.10: Energy levels within the band gap of $\mathrm{ZnS}$ for various dopants and accepts [92].

However, when a hole is trapped on a substitutional $\mathrm{Cu}^{+}$, these $3 \mathrm{~d}$ states split into e (lower) and $\mathrm{t}_{2}$ (upper) states with a splitting about $0.75-0.95 \mathrm{eV}$ [93].

When $\mathrm{Cl}$ or other co-activators $(\mathrm{Br}, \mathrm{I}, \mathrm{Al})$ are added together with $\mathrm{Cu}$, both the green and blue lines are observed depending on the $\mathrm{Cu}$ concentration and the $\mathrm{Cu} / \mathrm{Cl}$ ratio. The green line is emitted through the transition from a localized shallow donor state to a trapped hole on a $\mathrm{Cu}^{+}$substitutional defect ( $\mathrm{t}_{2}$ state). The blue line may come from SA centers or C3v symmetry structure [94]. The blue emission centers with C3v symmetry are proven to be consisted of one $\mathrm{Cu}$ atom, three nearest neighbor $\mathrm{S}$ atoms and one $\mathrm{S}$ vacancy with $\mathrm{Cu}$ displaced away from $\mathrm{S}$ vacancy [66]. However, the blue emission centers are still not well understood and need further research efforts. The brief illustration of various emission centers in $\mathrm{ZnS}$ is presented in Figure 2.10. 


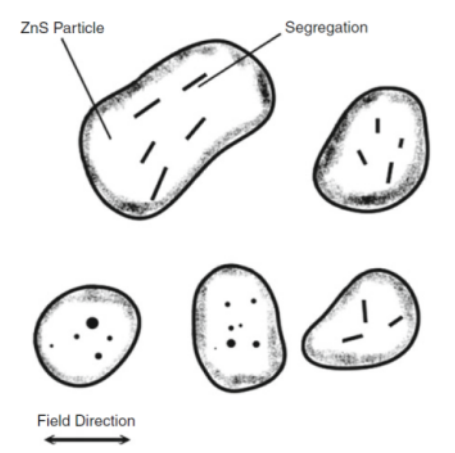

(a)

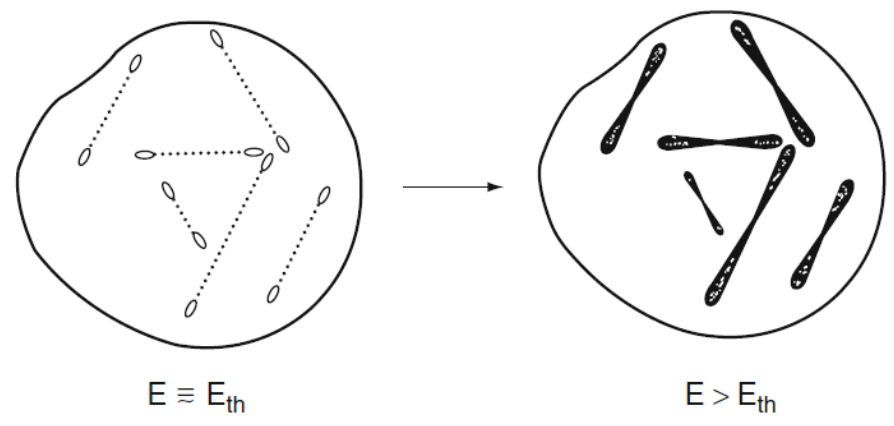

(b)

Figure 2.11: (a) Typical microscopic view of EL from $\mathrm{ZnS}: \mathrm{Cu}, \mathrm{Cl}$ particles. (b) Phosphor particles containing dark segregations and emitting spots. Double lines at threshold voltage and above the threshold voltage are illustrated [46].

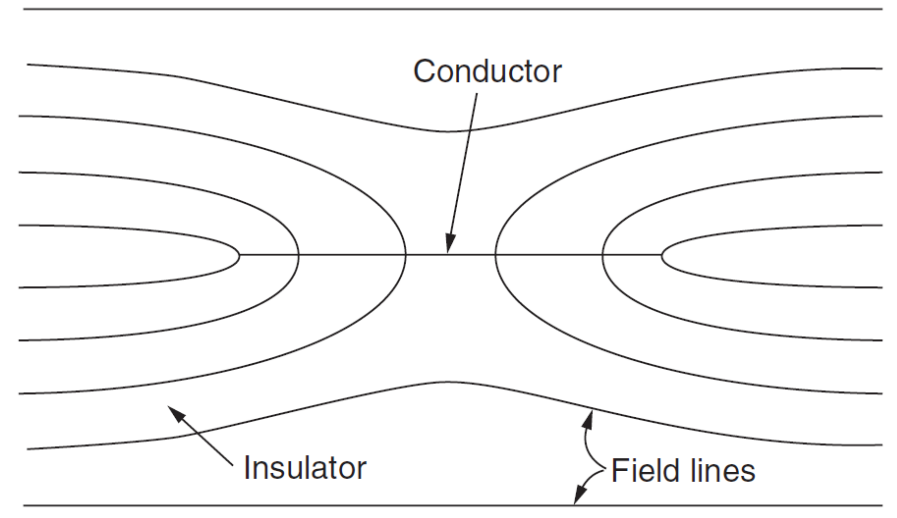

Figure 2.12: Illustration of the phenomenon of the electric field concentrated on the tips of the conducting needles [46].

\subsubsection{Light emission mechanisms}

\section{Fischer's bipolar field emission theory}

Although ACPEL phenomenon has been discovered for many years, its excitation and recombination mechanisms are still not fully established. Among all the proposed theories, the bipolar field emission model proposed by Fischer is so far the most acceptable one [45, 46]. As mentioned earlier, $\mathrm{Cu}_{2} \mathrm{~S}$ needles are observed in Fischer's experiment. A simplified illustration proposed by Fischer is given to represent the double comet structure of the emitting lines in Figure 2.11. Fischer postulated that small $\mathrm{Cu}_{2} \mathrm{~S}$ needles were formed 
during the fabricating process of $\mathrm{ZnS}$ particles and these needles lied across the particle with different orientations as shown in Figure 2.11(a). Due to the field enhancement effect, when the applied voltage exceeds the threshold value, lights are emitted around the lines to form the double comet shapes as shown in Figure 2.11(b).

According to the above observations, Fischer proposed the following ACPEL working principles. When an electric field is applied to the phosphor particles, relatively high electric fields will be concentrated on the tips of $\mathrm{Cu}_{2} \mathrm{~S}$ conducting needles (the effective tip radius is in the order of 100nm) compared to other regions in Figure 2.12. Therefore, an applied field of $10^{6}-10^{7} \mathrm{~V} / \mathrm{m}$ is able to induce a local field of $10^{8} \mathrm{~V} / \mathrm{m}$ or more following Equation 2.1.

$$
E=E_{0}\left(\frac{L}{d}\right)^{2}
$$

where $E_{0}$ is the electric field strength across the $\mathrm{ZnS}$ phosphor powders, $L$ the length of the $\mathrm{Cu}_{2} \mathrm{~S}$ needles and $d$ the diameter of the needle. This electric field is strong enough to induce tunneling of holes from one end of the needle and electrons from the other to the $\mathrm{ZnS}$ lattice. The field-emitted electrons are captured in shallow traps of the donor sites, while holes are trapped by the recombination centers (acceptor sites). When the field is reversed, the emitted electrons recombine with the trapped holes to produce EL emissions [46]. Figure 2.13 shows the illustrations of the basic principle of the bipolar field emission model.

This model could well explain the relationship between brightness and voltage/frequency [95], different active waveforms [95], delayed emission [96], polarization field effects [97], and temperature. However, according to this model, the electric field at the center of the needle is zero and this leads to no light emission at the center which does not agree with experimental results. It is observed that there is light emission all over the needle. The other aspect of Fischer's model that causes a lot of doubts is that only few reports claim to find the $\mathrm{Cu}_{\mathrm{x}} \mathrm{S}$ needles. One of these is a short article by Ono et al. [41] reporting the detection of copper sulphide needles in the form of $\mathrm{Cu}_{2} \mathrm{~S}$ by means of $\mathrm{X}$-ray absorption 

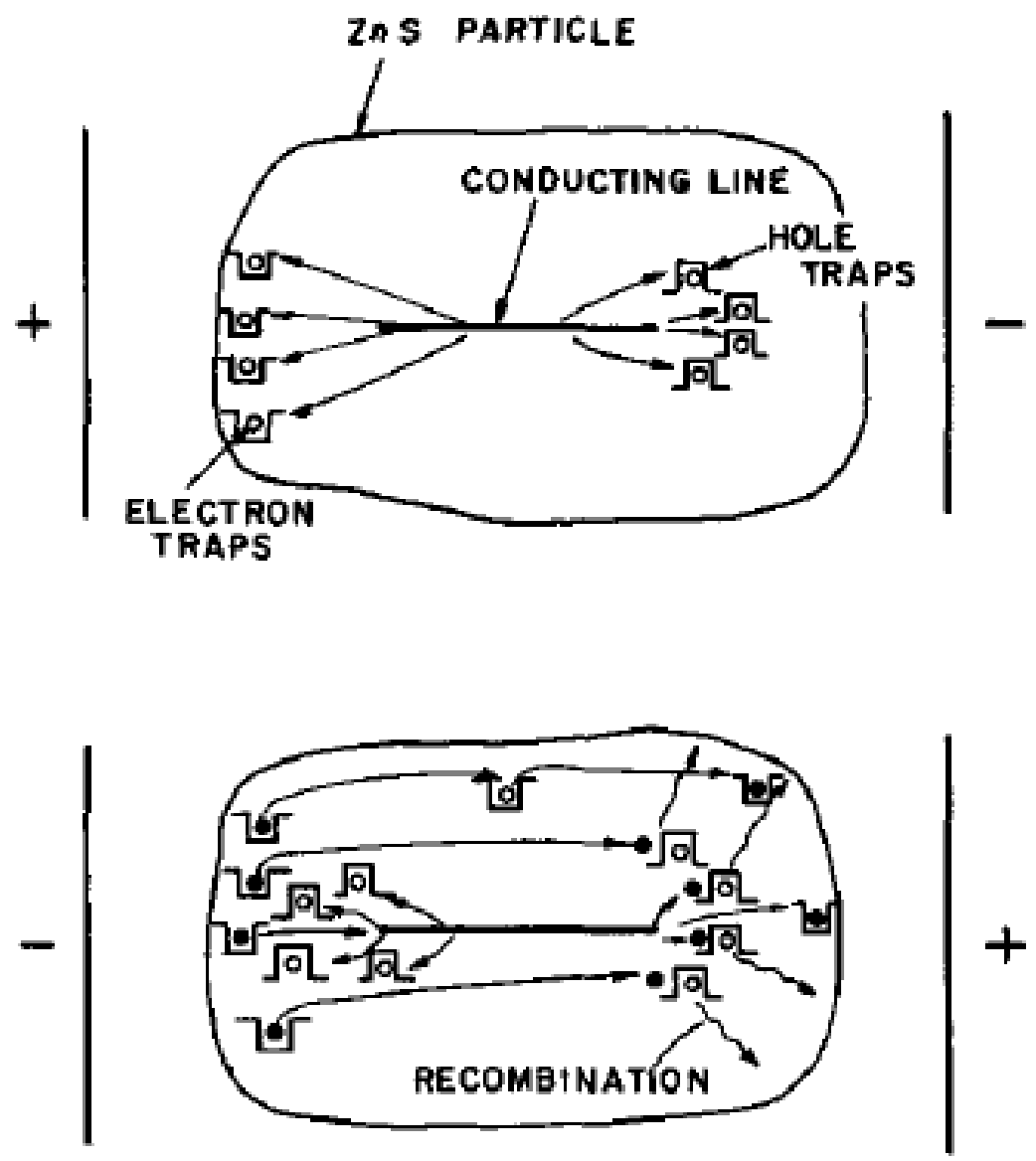

Figure 2.13: Illustration of the basic principle of the bipolar field model indicating the trap sites [46].

near edge structure (XANES). Another is a short report by Guo et al. [98] who observed a higher concentration of copper in the twin boundary areas and crystal streaks of sphalerite using high resolution electron microscopy and scanning electron microanalysis. A third study by Nien et al. [78] reported the observation of $50-80 \mathrm{~nm} \mathrm{Cu}_{\mathrm{x}} \mathrm{S}$ precipitates from X-ray mapping of a transmission electron microscope. The fourth is an article produced by Warkentin et al. [99] who claimed that copper sulphide was present in the form of tiny CuS-like clusters by means of extended X-ray absorption fine structure (EXAFS). However, others had found no evidence for copper sulphide needles and it was suggested that the EL generation process was related to the surface roughness of phosphor particles [80, 96]. Moreover, experiments done by Ireland and Silver [19] showed that the more aligned 
surface marks generates more intensive lights, suggesting that light emission was closely related to surface defects. Thus, the precise nature and roles of copper sulphide clusters and the carrier generation and recombination processes still remain open to conjecture.

\section{Junction model}

The junction model was proposed by Brovetto et al. [81] with the observation that the linear comet-shaped EL patterns were related to the defective borders at the boundary between regions of (001) planes. In this model, the defective borders containing broken covalent bonds are postulated to behave like a p-type semiconductor and the bulk $\mathrm{ZnS}$ is assumed to be n-type in nature. As shown in Figure 2.14, p-n junctions are formed at the boundaries between the defect borders and bulk $\mathrm{ZnS}$. The defective border is believed to have higher

conductivity than the bulk. As the electric field is directed as in Figure 2.14(b), electrons are injected at the left extremity as a direct-biased diode. At the right extremity, which, on the contrary, corresponds to a reverse-biased diode, electrons are extracted by bandto-band tunneling and holes are injected. When the field is reversed as shown in Figure 2.14(c), Electrons enter at the right extremity and are trapped, whilst holes, injected at the left extremity, recombine radiatively, through $\mathrm{Cu}$ centers, with a part of the electrons released from the traps.

With the consideration of space charge accumulation at the defective borders, this junction model is able to explain the brightness distribution along the comet shapes [100], electroluminescence build-up [101], and the brighness-voltage relationship [102]. However, the biggest doubt lies in the p-type $\mathrm{ZnS}$ in the bulk apart from the unidentified nature of the defective borders. Moreover, this junction model fails to explain the delayed emission and multiple emission peaks. 

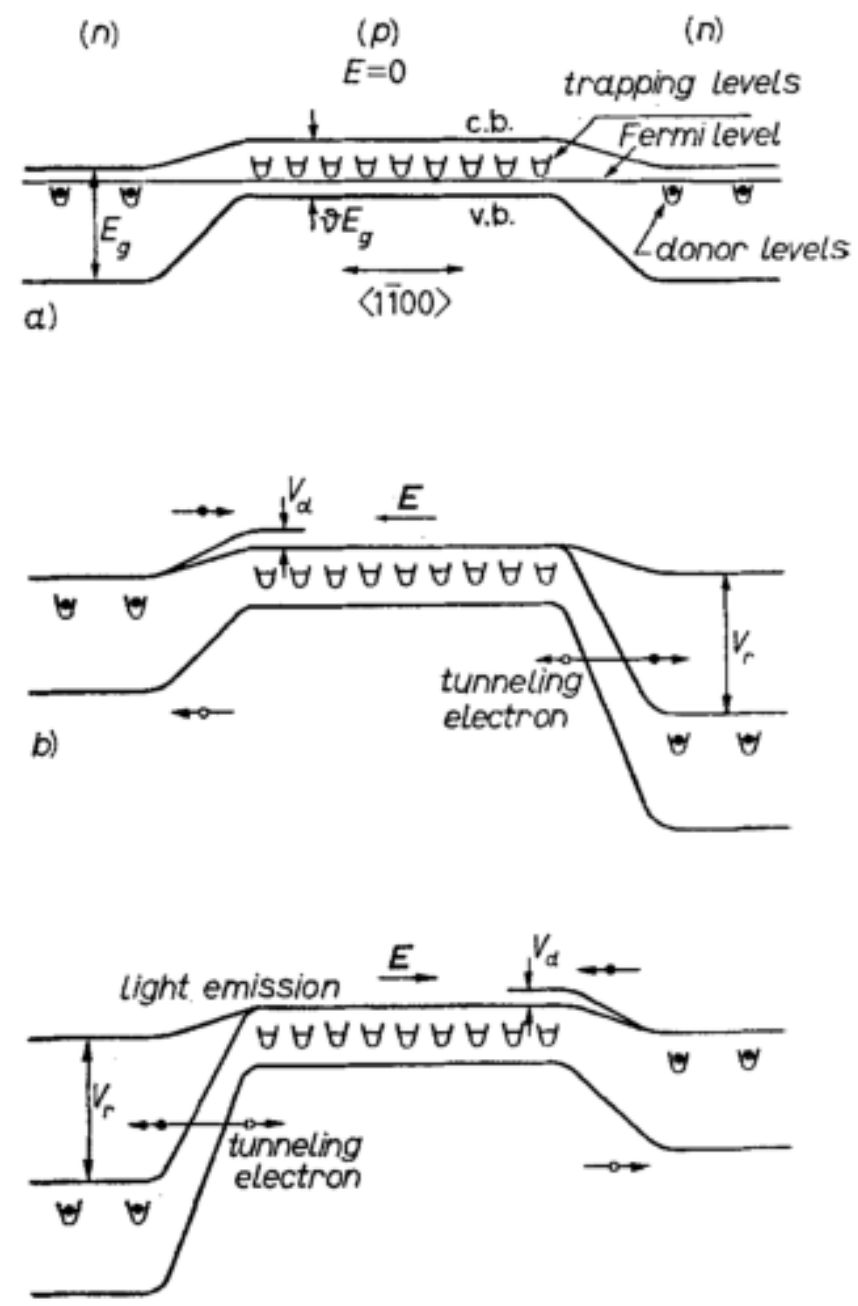

c)

Figure 2.14: Band behaviors at the contact between a defective border and the bulk $\mathrm{ZnS}$. Three situations are shown: (a) when no field is applied, (b) when the field is parallel to the border, and (c) when the field is directed in opposite senses [81].

\subsection{Printing and coating processes}

One of the major advantage of ACPEL devices is its simple and low cost printing process. Printing is defined as the reproduction of patterns by transfer of matter to a surface by mechanical force or hydrodynamic stress [103]. There are, in general, seven steps in most of printing processes: conditioning, fluid acquisition, predosing, dosing, transfer, relaxation and drying. 
Before the actual printing, a conditioning step is usually performed to cut the substrate to a desired size, clean substrate surface, or even UV or plasma treat the surface for better wetting capability. Thereafter, a fluid containing the desired printing substances is formulated in consideration of the viscosity and surface tension that are suitable for the specified printing process. Followed predosing and dosing steps then ensure the accurate amount and desired pattern of the fluid to be printed. Mechanical pressure, hydrodynamical shear, or intense chemical or thermodynamic forces can then be applied to transfer the liquid to the substrate surface. After the successful transfer, a surface-levelling process takes place, where the liquid-phase printed layer relaxes in the short period of time due to a hydrodynamical flow driven by capillary forces. The final drying step is for the complete removal of solvent residuals from the printed film.

\subsubsection{Drop casting}

A drop casting process is probably the simplest film-forming technique available. It is simple and does not require any equipment apart from a very horizontal work surface and pipettes. During a drop casting process, a specified amount of solution is dropped onto a substrate followed by relaxation and drying. While it is possible to prepare films of good quality and also thick films the technique suffers from a lack of control over the film thickness and often picture framing effects are observed near the edges of the film or precipitation during drying [104]. If the surface tension of the liquid dominates, the resulted film after drying will be inhomogeneous. Moreover, it is disadvantageous that the material to be coated is required to be highly soluble in the solvent used if crystallisation or precipitation is to be avoided.

\subsubsection{Flexography}

Flexography is mainly used in the packaging industry for the patterning of foil, bags, cardboards, etc. The principle of flexography is shown in Figure 2.15. There are two main 
units in flexography: a inking unit consisting of a fluid reservoir and an anilox roller and a printing unit consisting of a plate cylinder and an impression cylinder. The production process using flexography is briefly described as follows. Firstly, the printing fluid is prepared with adjusted viscosity and is placed in the fluid reservoir. Secondly, the rotating anilox roller is wetted by feeding the fluid from the ink feed chamber and excess fluid is wiped off by the doctor blade. After a defined amount of fluid is transported by the cell of the anilox roller, the fluid is transferred onto the printing plate, which is mounted on the plate cylinder and contains the printing layout. The patterned fluid is then transferred onto the substrate by mechanical force between the plate cylinder and the impression cylinder. Lastly, a relaxation and drying step is followed to form the final printed film. Thermal treatment is normally used for drying purpose. This process enables a fast printing speed up to $1000 \mathrm{~m} / \mathrm{min}$ at about $2 \mathrm{~m}$ printing width. Moreover, the printed layer can reach several tens of micrometers in thickness. Another important advantage is the clear distinction between printed and non-printed areas. However, fluid with a viscosity below $0.5 \mathrm{mPa} \cdot \mathrm{s}$ is not applicable for this process as the fluid will be spin off the printing plate during rotation. Due to the complexity of forces involved in this process, a halo effect is often observed, where a unfavorable line surrounds the printed pattern.

\subsubsection{Screen printing}

A flatbed screen printing press consists of a screen (mesh mounted in a frame), a squeegee (doctor blade), a flooding blade, and a substrate holder. The principle of screen printing is shown in Figure 2.16. The well prepared fluid is applied or dropped onto the screen and out of the patterning area. Subsequently, the effective region is flooded by the fluid using the flooding blade. The textures of the mesh for printing and nonprinting areas are different. The mesh at the nonprinting areas is covered with an impermeable stencil material. Only the fluid falling within the printing areas is able to penetrate through the mesh when pressed by the squeegee presses and comes in contact with the substrate. The transfer process takes 


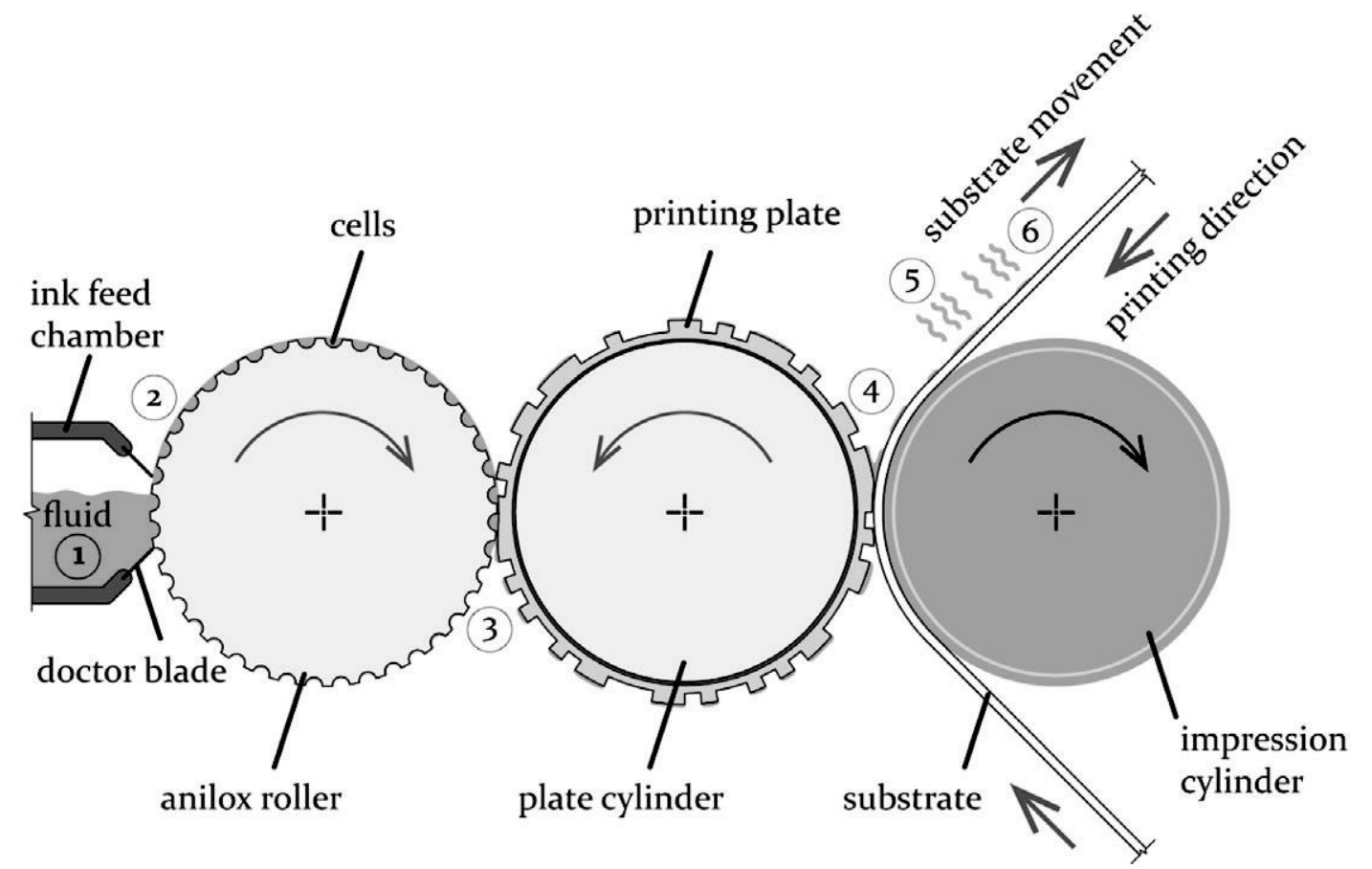

Figure 2.15: Principle of flexography [103].

place after the squeegee blade has passed across the screen and as the screen is lifted away from the substrate, followed by relaxation and drying steps.

Screen printing is a very robust process with the easy operation of squeezing ink through a stencil. It is possible to reproduce patterns on almost all kinds of surfaces (rough, even, plain, bent) and materials (e.g.,paper, cardboard, plastic, metal, wood, ceramics, glass, foils). Moreover, it does not have such high requirement of the viscosity of fluid compared to other techniques. With the proper combination of screen and fluid, even untrained people can succeed using screen printing to fabricate samples. However, one important concern to use screen printing process is the matching of the particle size and the mesh opening. As a rule of thumb, the mesh opening size should be at least three fold bigger than the particle size. Larger particles tend to block the mesh, which may also occur if the fluid dries too fast. In addition, there may be slurring effects due to a massive squeegee pressure or a slack mesh, which leads to the characteristic wavy edges. The other disadvantage of screen printing is its lower resolution or accuracy which is only limited to micrometer scale, compared 


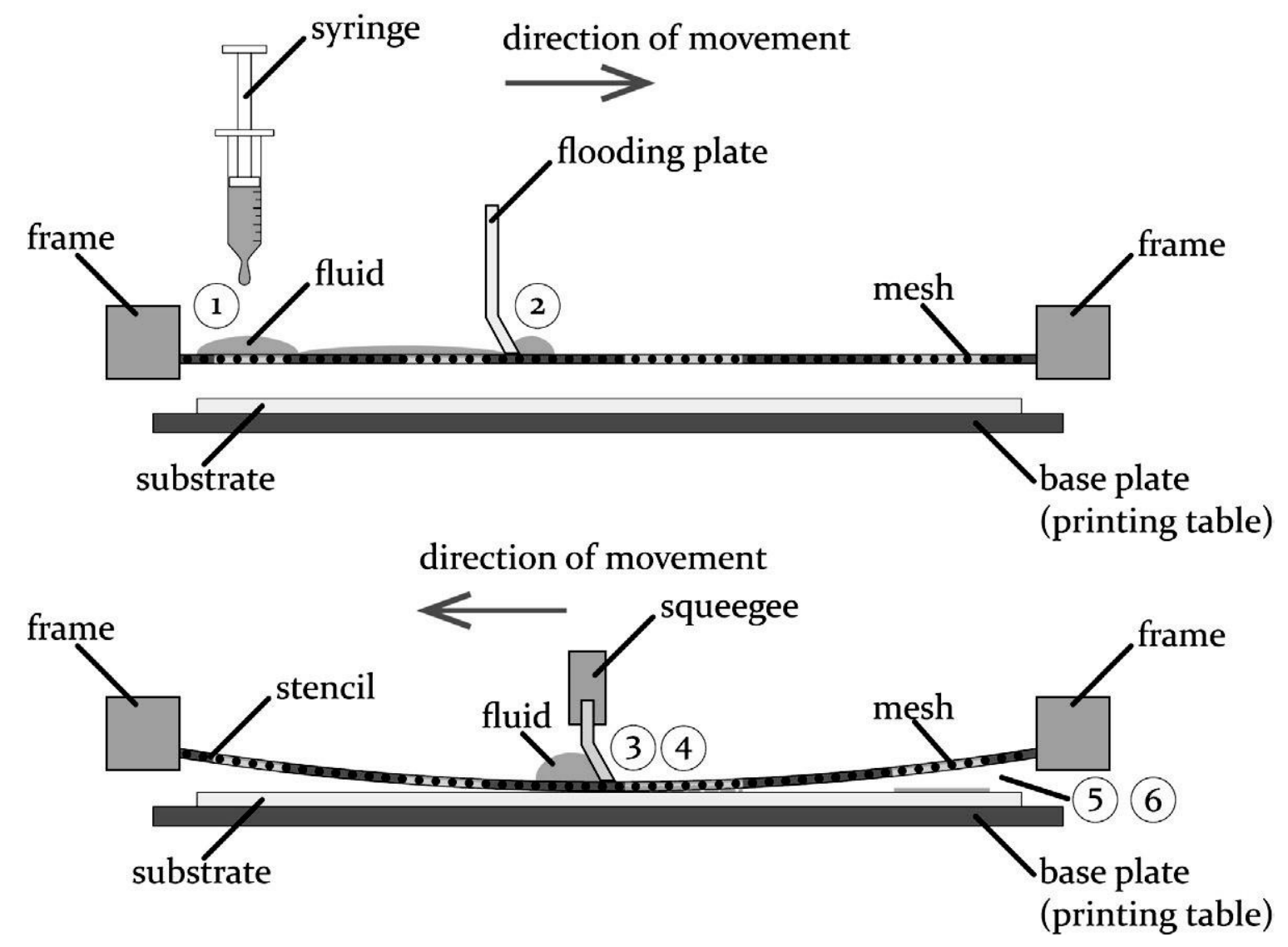

Figure 2.16: Principle of screen printing [103].

with other printing techniques.

\subsubsection{Spin coating}

As a highly reproducible lab-scale method, spin coating is normally used as a reference method for producing, for example, thin films of organic semiconductors and verifying the layer formation capabilities of printing formulations. Therefore, the required quantities of liquid are small, but it can only apply to a limited size of the substrates usually not more than a few centimeters in diameter. Figure 2.17 simply illustrates the principle of a spin coating process. A certain amount of the prepared liquid is first dropped in the center of the substrate, which is vacuum held onto an electrically driven spinning plate. The liquid drop is spread over the substrate surface by centrifugal forces with a specified spinning speed and duration, and excess liquid is thrown off the substrate from the rim. This process is easy 


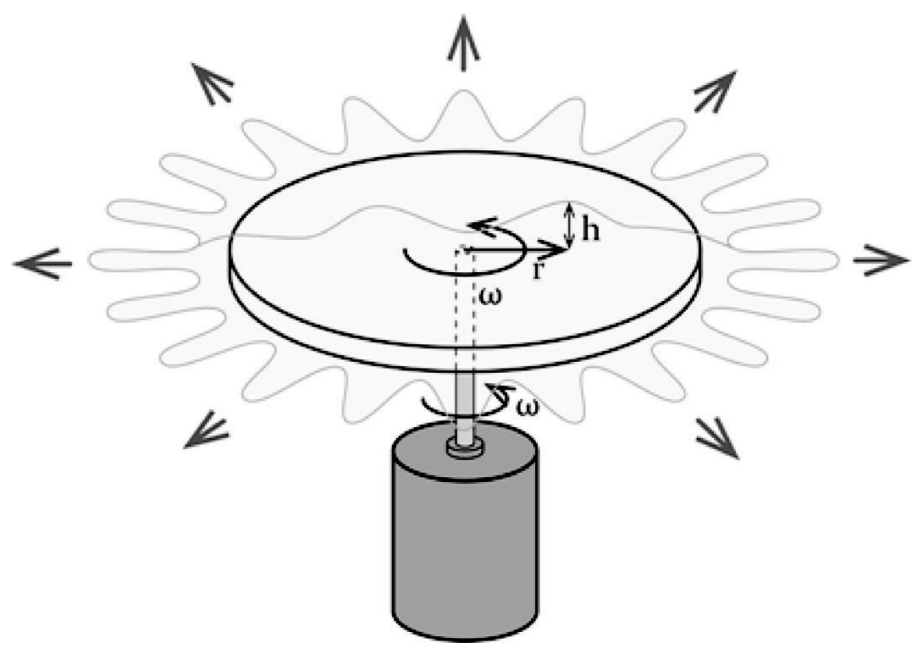

Figure 2.17: Principle of spin coating [103].

and straight forward. The thickness of coated layer can range from a few nanometers to a few micrometers depending on the viscosity of the liquid and the spinning speed and time. However, spin coating is not suitable for depositing liquids containing large particles. The particles will be thrown off the spinning plate, leading to missing spots or uneven surface.

If the evaporation from the liquid films can be neglected, the thickness of coated film can be determined through the following relationship [103]:

$$
h \propto \frac{60}{2 \pi n} \sqrt{\frac{3 \eta}{2 \rho t_{s}}}
$$

where $n$ is the rotational speed of the spin coater, $\eta$ the viscosity of the liquid, $\rho$ the mass density of the liquid (in $\mathrm{kg} / \mathrm{m}^{3}$ ) and $\mathrm{t}_{\mathrm{s}}$ the spinning time. If solvent evaporation is substantial, and provided that spinning is continued until the deposited film has dried by solvent evaporation, the thickness can be then estimated as [103]:

$$
h \propto \sqrt{\frac{60}{2 \pi n}}\left(\frac{3 k \eta}{2 \rho}\right)^{1 / 3}
$$

where $k$ is a solvent-specific constant proportional to the saturation vapor pressure in the gas atmosphere and to vapor diffusivity. 


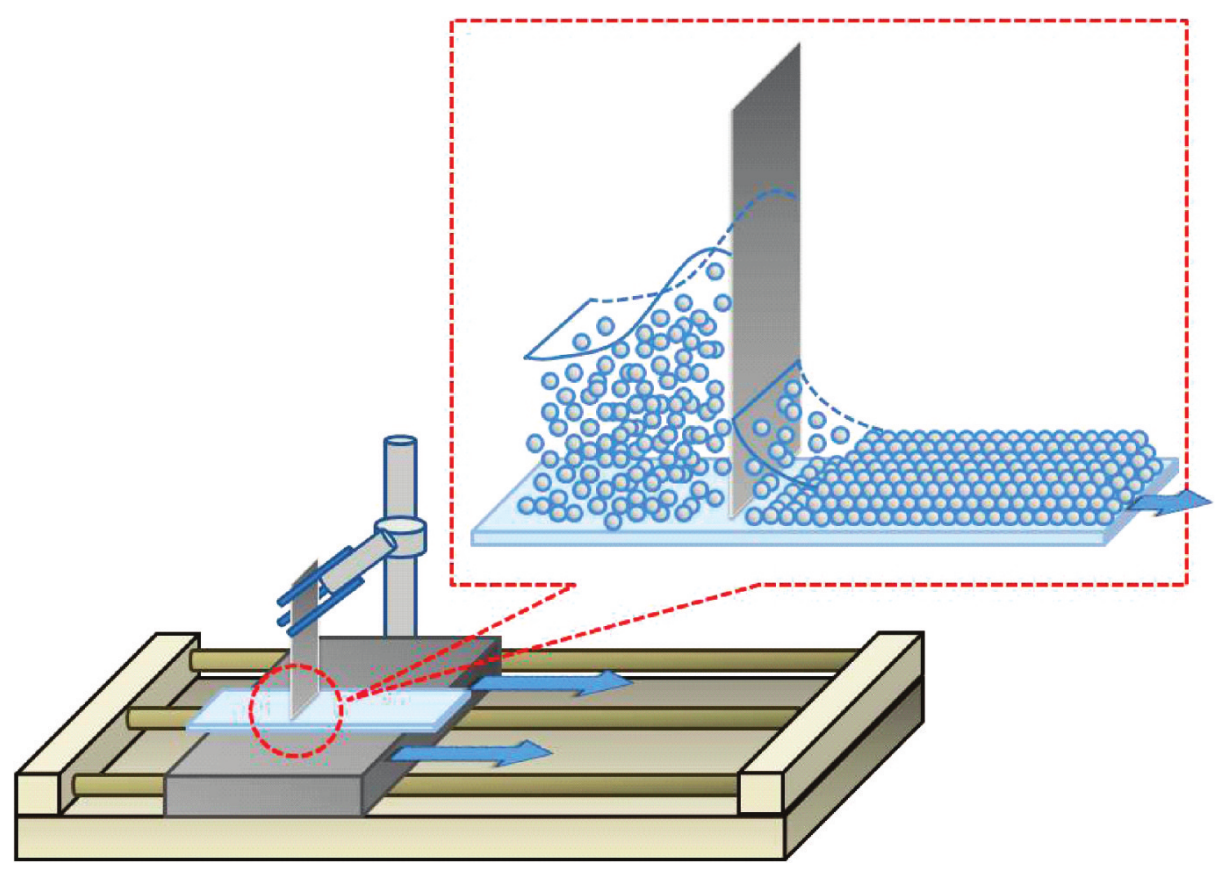

Figure 2.18: Principle of blade coating [105].

\subsection{5 blade coating}

Blade coating, also known as doctor blading or knife coating, uses a doctor blade or a knife instead of a die with a closed chamber. The working principle of a doctor blade is similar to that used in gravure printing or flexography, where the doctor blade is installed toward a roller. A blade coating process is illustrated in Figure 2.18. First, a certain amount of liquid is dropped onto the substrate and in front of the blade. With the razor blade gently touching the substrate, a film is formed by moving the blade across the substrate. The film thickness is determined by the blade type. The advantages of blade coating are its easy setup and operation as well as the low cost of the system. However, as the doctor blade coater is an open system, solvents is subjected to evaporation and hence the rheological behavior of the liquid will be altered. 


\subsection{Optical radiation metrology}

In order to fabricate warm white hybrid light source, it is necessary to quantify the warm white color. This involves the understanding of optical radiation metrology and the related parameters. Optical radiation metrology covers the areas of photometry, radiometry and colorimetry. Photometry is the science of the measurement of light, in terms of its perceived brightness to the human eye. Radiometry includes a set of techniques for measuring electromagnetic radiation, including visible light. As opposed to photometric techniques, radiometric techniques characterize the distribution of the radiation's power in space. Colorimetry is the science and technology used to quantify and describe physically the human color perception. These three categories of techniques form the basis of optical property measurements, which in this study includes the parameters of luminance, luminous efficacy and efficacy, CIE chromaticity, CCT, and color rendering index (CRI).

\subsubsection{Luminance}

Luminance is a photometric measure of the luminous intensity per unit area of light travelling in a given direction. It is a parameter quantifies the amount of light that is transmitted, emitted or reflected from a certain area and a defined solid angle. The SI unit for luminance is candela per square meter $\left(\mathrm{cd} / \mathrm{m}^{2}\right)$. The luminance indicates how much luminous power will be detected by an eye looking at the surface from a particular angle of view and is thus an indicator of how bright the surface will appear. The luminance of a specified point of a light source, in a specified direction is defined as:

$$
L_{v}=\frac{d^{2} \Phi_{v}}{d \Sigma d \Omega_{\Sigma} \cos \theta_{\Sigma}}
$$

where $d^{2} \Phi_{v}$ is the luminous flux leaving the area $d \Sigma$ in any direction contained inside the solid angle $d \Omega_{\Sigma}, d \Sigma$ an infinitesimal area of the source containing the specified point, $d \Omega_{\Sigma}$ an infinitesimal area of the source containing the specified direction, $\theta_{\Sigma}$ the angle between 
the normal $n_{\Sigma}$ to the surface $d \Sigma$ and the specified direction.

\subsubsection{Luminous efficacy and efficiency}

Luminous efficacy is a measure of how well a light source produces visible light. It is the ratio of luminous flux to power, measured in lumens per watt in SI. The luminous efficacy of a source is the product of how well it converts energy to electromagnetic radiation, and how well the emitted radiation is detected by the human eye. Therefore luminous efficacy, $k$, is denoted as:

$$
k=\frac{\Phi_{v}}{\Phi_{e}}=\frac{\int_{0}^{\infty} K(\lambda) \Phi_{e, \lambda} d \lambda}{\int_{0}^{\infty} \Phi_{e, \lambda} d \lambda}
$$

where $\Phi_{e}$ is the radiant flux, $\Phi_{e, \lambda}$ the spectral radiant flux, $K(\lambda)=K_{m} V(\lambda)$ the spectral luminous efficacy. Photopic luminous efficacy of radiation has a maximum possible value of $683 \mathrm{~lm} / \mathrm{W}$, for the case of monochromatic light at a wavelength of $555 \mathrm{~nm}$ (green). When luminous efficacy is normalized by the maximum value, the resulted dimensionless quantity is then called luminous efficiency.

\subsubsection{CIE chromaticity}

The CIE 1931 color spaces created by CIE in 1931 were the first defined quantitative links between physical pure colors (i.e. wavelengths) in the electromagnetic visible spectrum, and physiological perceived colors in human color vision. The CIE XYZ color space encompasses all color sensations that an average person can experience. That is why CIE $\mathrm{XYZ}$ (Tristimulus values) is a device invariant color representation. When judging the relative luminance (brightness) of different colors in well-lit situations, humans tend to perceive light within the green parts of the spectrum as brighter than red or blue light of equal power. The luminosity function that describes the perceived brightnesses of different wavelengths is thus roughly analogous to the spectral sensitivity of M cones. The CIE model capitalises on this fact by defining $\mathrm{Y}$ as luminance. $\mathrm{Z}$ is quasi-equal to blue stimulation, or the $\mathrm{S}$ cone response, and $\mathrm{X}$ is a mix (a linear combination) of cone response curves 
chosen to be nonnegative. The XYZ tristimulus values are thus analogous to, but different from, the LMS cone responses of the human eye. Defining Y as luminance has the useful result that for any given $\mathrm{Y}$ value, the $\mathrm{XZ}$ plane will contain all possible chromaticities at that luminance. The unit of the tristimulus values $\mathrm{X}, \mathrm{Y}$, and $\mathrm{Z}$ is often arbitrarily chosen so that $\mathrm{Y}=1$ or $\mathrm{Y}=100$ is the brightest white that a color display supports. The tristimulus values for a color with a spectral radiance $L_{e, \Omega, \lambda}$ are given in terms of the standard observer by:

$$
\begin{aligned}
& X=\int_{380}^{780} L_{e, \omega, \lambda} \bar{x}(\lambda) d \lambda \\
& Y=\int_{380}^{780} L_{e, \omega, \lambda} \bar{y}(\lambda) d \lambda \\
& X=\int_{380}^{780} L_{e, \omega, \lambda} \bar{z}(\lambda) d \lambda
\end{aligned}
$$

where $\lambda$ is the wavelength of the equivalent monochromatic light (measured in nanometers).

Since the human eye has three types of color sensors that respond to different ranges of wavelengths, a full plot of all visible colors is a three-dimensional figure. However, the concept of color can be divided into two parts: brightness and chromaticity. The CIE XYZ color space was deliberately designed so that the Y parameter is a measure of the luminance of a color. The chromaticity of a color is then specified by the two derived parameters $\mathrm{x}$ and $y$, two of the three normalized values being functions of all three tristimulus values $\mathrm{X}$, $\mathrm{Y}$, and $\mathrm{Z}$ :

$$
\begin{aligned}
& x=\frac{X}{X+Y+Z} \\
& y=\frac{Y}{X+Y+Z}
\end{aligned}
$$




$$
z=\frac{Z}{X+Y+Z}=1-x-y
$$

The derived color space specified by $\mathrm{x}, \mathrm{y}$, and $\mathrm{Y}$ is known as the CIE xyY color space and is widely used to specify colors in practice.

\subsubsection{Color correlated temperature}

The color temperature of a light source is the temperature of an ideal black-body radiator that radiates light of comparable color to that of the light source. Color temperatures over $5000 \mathrm{~K}$ are called cool colors (bluish white), while lower color temperatures (2700-3000 K) are called warm colors (yellowish white through red). Warm in this context refers to radiated heat flux rather than temperature; the spectral peak of warm-coloured light is closer to infra-red and most natural warm-coloured light sources emit significant infra-red radiation.

The color temperature of the electromagnetic radiation emitted from an ideal black body is defined as its surface temperature in kelvins, or alternatively in mireds (micro-reciprocal kelvins). Many other light sources, such as fluorescent lamps, or LEDs (light emitting diodes) emit light primarily by processes other than thermal radiation. This means that the emitted radiation does not follow the form of a black-body spectrum. These sources are assigned what is known as a correlated color temperature (CCT), which is useful only for white or generally whitish light sources. CCT is the color temperature of a black-body radiator which to human color perception most closely matches the light from the lamp. If a narrow range of color temperatures is considered, those encapsulating daylight being the most practical case, one can approximate the Planckian locus in order to calculate the CCT in terms of chromaticity coordinates. The Planckian locus is the path or locus that the color of an incandescent black body would take in a particular chromaticity space as the 
blackbody temperature changes. McCamy proposed this cubic approximation [106]:

$$
C C T(x, y)=-449 n^{3}+3525 n^{2}-6823.3 n+5520.33
$$

where

$$
n=\frac{x-x_{e}}{y-y_{e}}
$$

where $x_{e}=0.332$ and $y_{e}=0.1858$.

\subsubsection{Color rendering index}

A CRI is a quantitative measure of the ability of a light source to reveal the colors of various objects faithfully in comparison with an ideal or natural light source. Light sources with a high CRI are desirable in color-critical applications. The CRI of a light source does not indicate the apparent color of the light source but is determined by the light source's spectrum. A more continuous spectrum produces a higher CRI. CRI can be calculated from eight reference samples in CIE color space when illuminated by a given light source. First, colorimetric data must be transformed from CIE 1931 values $(\mathrm{X}, \mathrm{Y}, \mathrm{Z}, \mathrm{x}, \mathrm{y})$ to the $(\mathrm{u}, \mathrm{v})$ coordinate of the 1960 diagram by means of the following:

$$
\begin{aligned}
& u=\frac{4 X}{X+15 Y+3 Z} \\
& v=\frac{6 Y}{X+15 Y+3 Z}
\end{aligned}
$$

Secondly, to account for the adaptive color shift due to the different state of chromatic adaptation under the lamp to be tested, $\mathrm{k}$, and under the reference illuminant, $\mathrm{r}$, use the following formula:

$$
u_{k, i}^{\prime}=\frac{10.872+0.404 \frac{c_{r}}{c_{k}} c_{k, i}-4 \frac{d_{r}}{d_{k}} d_{k, i}}{16.518+1.481 \frac{c_{r}}{c_{k}} c_{k, i}-\frac{d_{r}}{d_{k}} d_{k, i}}
$$




$$
v_{k, i}^{\prime}=\frac{5.520}{16.518+1.481 \frac{c_{r}}{c_{k}} c_{k, i}-\frac{d_{r}}{d_{k}} d_{k, i}}
$$

where $i$ is a tested color sample and c and d follow the below formulas:

$$
\begin{gathered}
c=\frac{4-u-10 v}{v} \\
d=\frac{1.708 v+0.404-1.481 u}{v}
\end{gathered}
$$

Color imetric data must now be transformed into the 1964 Uniform Space coordinates by the following:

$$
\begin{aligned}
& W_{r, i}^{*}=25\left(Y_{r, i}\right)^{1 / 3}-17 \\
& U_{r, i}^{*}=13 W_{r, i}^{*}\left(u_{r, i}-u_{r}\right) \\
& V_{r, i}^{*}=13 W_{r, i}^{*}\left(v_{r, i}-v_{r}\right) \\
& W_{k, i}^{*}=25\left(Y_{k, i}\right)^{1 / 3}-17 \\
& U_{k, i}^{*}=13 W_{k, i}^{*}\left(u_{k, i}-u_{k}\right) \\
& V_{k, i}^{*}=13 W_{k, i}^{*}\left(v_{k, i}-v_{k}\right)
\end{aligned}
$$

The difference between the resultant color shift of the test color sample under the test 
lamp $\mathrm{k}$ and illuminated by the reference $\mathrm{r}$ is then:

$$
\begin{gathered}
\triangle E_{i}=\sqrt{\left(\triangle U_{i}^{*}\right)^{2}+\left(\triangle V_{i}^{*}\right)^{2}+\left(\triangle W_{i}^{*}\right)^{2}} \\
C R I=\frac{1}{8} \sum_{i=1}^{8} R_{i}
\end{gathered}
$$

\subsection{Dielectric impedance spectroscopy}

\subsubsection{Fundamentals of impedance spectroscopy}

\section{Dielectrics and capacitors}

As the ACPEL device is sandwiched by two electrodes with dielectric materials in between, it can be considered as a parallel capacitor. A capacitor is characterized by its capacitance, which represents its capacity to store electrical energy. For an ideal parallel capacitor with vacuum in between, the capacitance is denoted as:

$$
C_{0}=\frac{\varepsilon_{0} A}{d}
$$

where $\varepsilon_{0}$ is the vacuum permittivity with the value of $8.854 \times 10^{-12} \mathrm{~F} . \mathrm{m}^{-1}$. $A$ is the area of the capacitor and $d$ is the distance between the parallel electrodes. When a dielectric material is inserted into a parallel capacitor, the capacitance becomes:

$$
C=\frac{\varepsilon_{r} \varepsilon_{0} A}{d}
$$

where $\varepsilon_{\mathrm{r}}$ is the relative permittivity of the dielectric material. From Equation 2.29, the device capacitance is largely dependent on the dielectric material. Materials with high relative permittivity will increase the capacitance largely. Microscopically, the effect of an inserted dielectric material on the overall capacitance can be explained by the dipole 


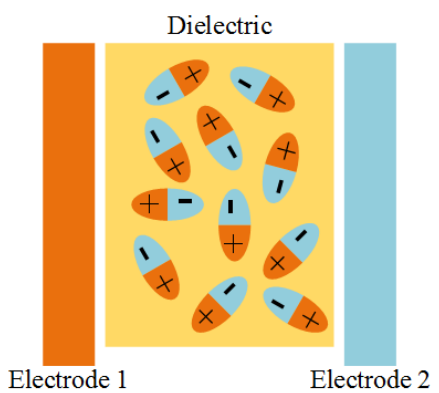

(a)

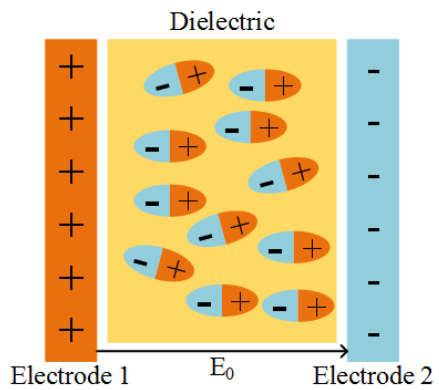

(b)

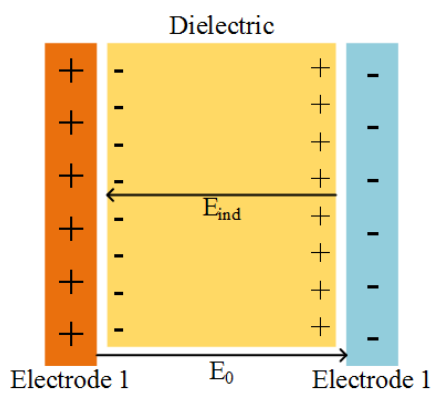

(c)

Figure 2.19: (a) dielectric material in a capacitor with randomly distributed dipoles. (b) An electric field is applied to the dielectric and dipoles are aligned. (c) Details of the electric field inside the dielectric.

alignment in an electric field as shown in Figure 2.19. When no electric field is applied over the dielectric-inserted capacitor, the dipoles inside the dielectric distributed randomly inside the material as shown in Figure 2.19(a). Upon the applying of an electric field, the dipoles align with the direction of the electric field $E_{0}$. If the molecules of the dielectric are nonpolar, the electric field produces an induced polarization in the molecule. These induced dipole moments tend to align with the external field, and the dielectric is polarized as shown in Figure 2.19(b). Therefore, a dielectric can be polarized by an external field regardless of whether the molecules in the dielectric are polar or nonpolar [107]. The net effect on the dielectric is the formation of an induced positive surface charge on the right face and an equal-magnitude negative surface charge density on the left face as shown in Figure 2.19(c). Thus the induced surface charges on the dielectric give rise to an induced electric field $E_{\text {ind }}$ in the direction opposite to the external field $E_{0}$.

\section{Polarization}

When a dielectric material is placed in an electric field, the electric dipoles will rearrange themselves. This process is known as polarization. Polarization can be classified into four categories: electronic, ionic, dipolar and interfacial.

Electronic or optical polarization process occurs in neutral atoms where the electron 
cloud is displaced relative to the nucleus by an external electric field. Thus electronic polarization is an induced polarization effect, characterized by the electronic polarizability $\alpha_{\mathrm{e}} . \alpha_{\mathrm{e}}$ is a microscopic polarization phenomenon that occurs in all materials and is one of the main mechanism that drives dielectric polarization. Electronic polarizability is defined as the dipole moment induced per unit electric field strength and is a measure of the ease with which the charge centers may be dislocated. With the approximation that the charge is spread uniformly in a spherical volume of radius $R$ of the atom, the dipole moment induced in the atom is:

$$
\mu_{e}=\left(4 \pi \varepsilon_{0} R^{3}\right) E
$$

Thus the electronic polarizability is:

$$
\alpha_{e}=4 \pi \varepsilon_{0} R^{3}
$$

Ionic polarization, also called atomic polarization, is observed when different atoms that comprise a molecule share their electrons asymmetrically, and cause the electron cloud to be shifted towards the stronger binding atom. The atoms acquire charges of opposite polarity and an external field acting on these net charges tends to change the equilibrium positions of the atoms themselves, leading to ionic polarization.

When a bond is formed between two molecules by the transfer of some valence electrons, a permanent dipole moment will originate in them. This permanent dipole moment is equal to the product of the charges of the transferred valence electrons and the inter-atomic distance between them. In the absence of an electric field, the dipole moment is cancelled out by thermal agitation resulting in a net zero dipole moment per molecule. In the presence of an electric field, the molecules carrying a permanent dipole moment will orient to align along the direction of the electric field. This process is called dipolar or orientational polarization. This occurs only in polar materials possessing permanent dipole moments. 
The dipolar polarizability per molecule is defined by the Debye equation,

$$
\alpha_{d}=\frac{\mu_{d}^{2}}{3 k T}
$$

where $\mu_{\mathrm{d}}$ is the inherent dipole moment due to the molecule structure and $k$ is the Boltzmann constant, $1.38 \times 10^{-23} \mathrm{~J} . \mathrm{K}^{-1}$. Note that unlike electronic polarizability and ionic polarizability, dipolar polarizability is temperature dependent. This is an important factor to consider when choosing a dielectric material for electronic and optical applications.

Space charge polarization involves the accumulation of charges at the grain boundaries, interface boundaries or electrode/material interfaces. An external electric field causes these charge dipoles to align. It is present in dielectric materials which contain charge carriers that can migrate for some distance through the bulk of the material (via diffusion, fast ionic conduction or hopping, etc.) thus creating a macroscopic field distortion. Such a distortion appears to an outside observer as an increase in the capacitance of the sample and may be indistinguishable from the real arise of the dielectric permittivity. Space charge polarization is the only type of polarization that is accompanied by a macroscopic charge transport. In

general, space charge polarization can be grouped into hopping polarization and interfacial polarization. In dielectric materials, localized charges (ions and vacancies, or electrons and holes) can hop from one site to another, which creates hopping polarization. Similarly, the separation of the mobile positive and negative charges under an electric field can produce an interfacial polarization. After the electric field is removed, charge carriers give a partial recovery and typically leave a finite polarization in the system.

\section{Dielectric relaxation}

In the previous reviews, dielectric polarization under DC electric field was discussed. However, the dielectric spectroscopy technique is conducted under AC electric field where dielectric relaxation takes place. Dielectric relaxation is the movement and alignment of 
dipoles and electric charges in response to the external alternating electric field.

Experimentally, the dielectric impedance is measured using excitation AC voltage signal with small amplitude $V_{A}$ applied at frequency $f$ :

$$
V(t)=V_{A} \sin (\omega t+\delta)
$$

where $\omega$ is the angular frequency with $\omega=2 \pi f$. In a linear or pseudolinear system, the current response to a sinusoidal voltage input will be a sinusoid at the same frequency but shifted in phase, denoted as:

$$
I(t)=I_{A} \sin (\omega t+\delta)
$$

Where $\delta$ is the phase angle and $I_{A}$ is the induced current amplitude. By using Eulers relationship, the $\mathrm{AC}$ voltage and current can be expressed as a complex function as follows:

$$
\begin{gathered}
V(t)=V_{A} e^{j \omega t} \\
I(t)=I_{A} e^{j \omega t-j \delta}
\end{gathered}
$$

Therefore, the impedance is then represented as a complex number with the combination of real $Z$ and imaginary $Z$ parts:

$$
Z^{*}=\frac{V_{A}}{I_{A}}=Z_{A} e^{j \delta}=Z_{A}(\cos \delta+j \sin \delta)=Z^{\prime}+j Z^{\prime \prime}
$$

Where $Z_{A}$ is the absolute impedance. The dissipation factor is given by:

$$
\tan \delta=\frac{Z^{\prime \prime}}{Z^{\prime}}
$$

Just as complex impedance, the dielectric data can be expressed in terms of complex permittivity $\left(\varepsilon^{*}\right)$, complex conductivity $\left(\sigma^{*}\right)$ and complex modulus $\left(M^{*}\right)$ with the respec- 
tive real and imaginary parts.

$$
\begin{gathered}
\varepsilon^{*}=\varepsilon^{\prime}-j \varepsilon^{\prime \prime} \\
\sigma^{*}=\sigma^{\prime}+j \sigma^{\prime \prime}=j \omega \varepsilon_{0} \varepsilon^{*} \\
M^{*}=M^{\prime}+j M^{\prime \prime}=\frac{1}{\varepsilon^{*}}=j \omega C_{0} Z^{*}
\end{gathered}
$$

By substituting Equation 2.37 into Equations 2.39-2.41, we can derive real and imaginary permittivity, conductivity and modulus from real and imaginary impedance:

$$
\begin{gathered}
\varepsilon^{\prime}=\frac{-Z^{\prime \prime}}{\omega C_{0}\left(Z^{\prime 2}+Z^{\prime \prime 2}\right)} \\
\varepsilon^{\prime \prime}=\frac{Z^{\prime}}{\omega C_{0}\left(Z^{\prime 2}+Z^{\prime \prime 2}\right)} \\
\sigma^{\prime}=\omega \varepsilon_{0} \varepsilon^{\prime \prime} \\
\sigma^{\prime \prime}=\omega \varepsilon_{0} \varepsilon^{\prime} \\
M^{\prime}=-\omega C_{0} Z^{\prime \prime} \\
M^{\prime \prime}=\omega C_{0} Z^{\prime}
\end{gathered}
$$

In dielectric materials, $\varepsilon^{\prime}$ represents the alignment of dipoles, which is the energy storage component that is an inverse equivalent of $Z$ ". $\varepsilon$ " represents the ionic conduction component that is an inverse equivalent of $Z^{\prime} . \varepsilon^{\prime \prime}$ is also called the loss factor. Dispersion, or 
frequency dependence, according to the laws of relaxation, is the corresponding frequency domain expression of complex permittivity, conductivity or modulus as a function of radial frequency. The dielectric permittivity dispersion is the relaxation of the electric displacement vector under the constraint of constant electric field while the modulus dispersion describes the relaxation of the electric field under the constraint of a constant displacement vector. That is why the complex modulus is the inverse of complex permittivity. In dielectrics containing mobile charges, it is more advantageous to use complex modulus so that variations in the large values of permittivity and conductivity at low frequencies are minimized. In such cases, the conductivity is composed of AC and DC parts. Therefore, the total conductivity is given by:

$$
\sigma_{T}=\sigma_{a c}+\sigma_{d c}=\omega \varepsilon_{0} \varepsilon^{\prime \prime}+\sigma_{d c}
$$

\section{Debye relaxation}

When a direct voltage applied to a dielectric for a sufficiently long duration is suddenly removed, the decay of polarization to zero value is not instantaneous but takes a finite time. This is the time required for dipoles to revert to a random distribution, in equilibrium with the temperature of the medium, from a field oriented alignment. Similarly, the build up of polarization following the sudden application of a direct voltage takes a finite time interval before the polarization attains its maximum value. This phenomenon is described by the general term dielectric relaxation. The polarization builds up from zero to a final value by a DC voltage according to an exponential law:

$$
P(t)=P_{\infty}\left(1-e^{\frac{t}{\tau}}\right)
$$


where $P(t)$ is the polarization at time $t$ and $\tau$ is called the relaxation time. $\tau$ is a function of temperature and is independent of time. The expression for Debye type behavior is:

$$
\varepsilon *=\varepsilon_{\infty}+\frac{\varepsilon_{s}-\varepsilon_{\infty}}{1+j \omega \tau}
$$

where $\varepsilon_{\mathrm{s}}$ is the static dielectric constant and $\varepsilon_{\infty}$ is the dielectric constant at very high frequency. Therefore,

$$
\begin{gathered}
\varepsilon^{\prime}=\varepsilon_{\infty}+\frac{\varepsilon_{s}-\varepsilon_{\infty}}{1+\omega^{2} \tau^{2}} \\
\varepsilon^{\prime \prime}=\frac{\left(\varepsilon_{s}-\varepsilon_{\infty}\right) \omega \tau}{1+\omega^{2} \tau^{2}}
\end{gathered}
$$

Cole and Cole showed that in a material exhibiting Debye relaxation each point corresponding to a particular frequency yields a semi-circle in the $\varepsilon^{\prime \prime}-\varepsilon^{\prime}$ plot [108]. This plot is called Cole-Cole plot or Nyquist plot. In the case of Debye relaxation, the points in Nyquist plot follows the following relationship:

$$
\left(\varepsilon^{\prime}-\frac{\varepsilon_{s}+\varepsilon_{\infty}}{2}\right)^{2}+\left(\varepsilon^{\prime \prime}\right)^{2}=\left(\frac{\varepsilon_{s}-\varepsilon_{\infty}}{2}\right)^{2}
$$

In studies involving the minimizing the space charge effects, electric modulus is preferred. The expressions for the real and imaginary modulus following Debye type relaxation are [109]:

$$
\begin{aligned}
& M^{\prime}=M_{\infty} M_{s} \frac{M_{\infty}+M_{s} \omega^{2} \tau^{2}}{M_{\infty}^{2}+M_{s}^{2} \omega^{2} \tau^{2}} \\
& M^{\prime \prime}=M_{\infty} M_{s} \frac{\left(M_{\infty}-M_{s}\right) \omega \tau}{M_{\infty}^{2}+M_{s}^{2} \omega^{2} \tau^{2}}
\end{aligned}
$$




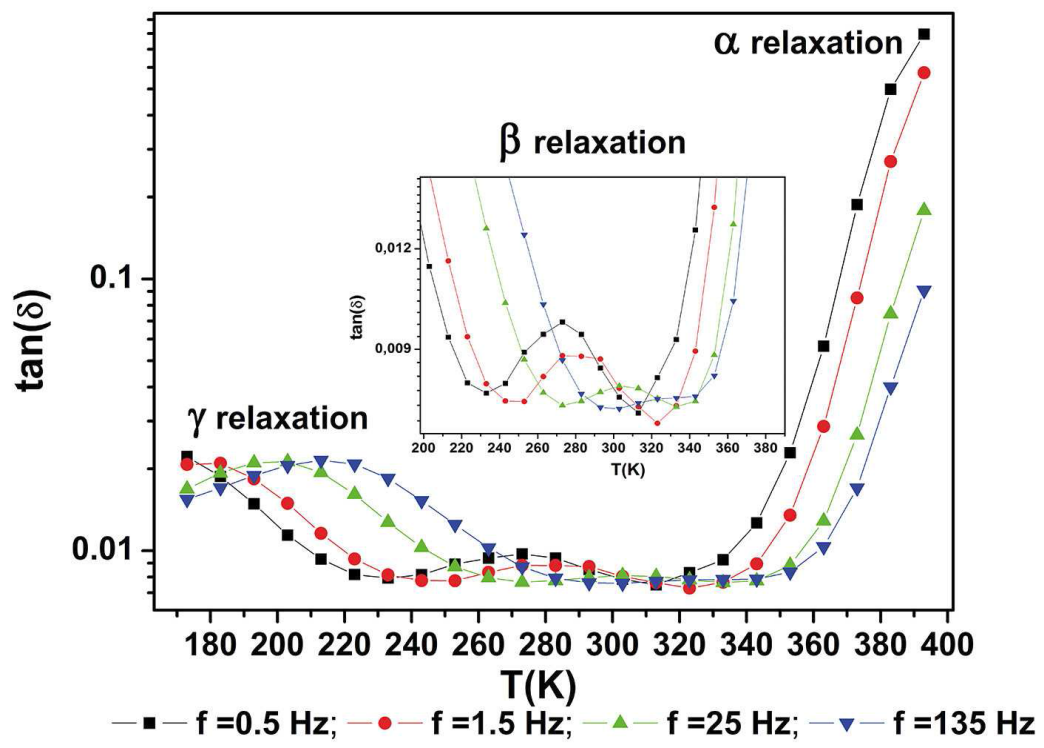

Figure 2.20: Loss tangent versus temperature at different frequencies. The inset shows a magnification region of the $\beta$ relaxation [112].

\subsubsection{Dielectric properties of resin}

The binder used in our experiment contains a crosslinking epoxy resin and a polyurethane film former. Epoxy resin are low molecular-weight pre-polymers or higher molecularweight polymers which normally contain at least two epoxide groups. During curing, epoxies generally undergo step reaction to form network polymer chains, which will have strengthened thermal and structural stability [110]. Due to their low concentration of free charge carriers, epoxy resins are considered as insulators. Therefore, their electrical characteristics are mainly attributed to the dielectric relaxation phenomena in response to an $\mathrm{AC}$ electric field, which are related to either dipolar orientation of polymer chains or migration of space charges [111].

In general, there may be three relaxation processes $\alpha, \beta$ and $\gamma$ within epoxy resin based polymers due to its polar nature. $\alpha$ relaxation occurs at high temperatures and low frequencies while $\beta$ and $\gamma$ relaxations take place at low temperatures and high frequencies as indicated in Figure $2.20[112,113]$. The $\alpha$ relaxation, also known as the primary or glass-rubber relaxation, is associated with the translational movement of the main polymer 
chains. Moreover, $\alpha$ relaxation is normally more significant at temperature above glass transition temperature $\left(\mathrm{T}_{\mathrm{g}}\right)$ [114]. $\beta$ and $\gamma$ relaxations, also known as secondary relaxations, can be detected at higher frequencies and lower temperatures than $\alpha$ relaxation. $\beta$ relaxation is caused by the reorientation of polar side groups while $\gamma$ relaxation is ascribed to the rearrangement of small parts of the polymer chains.

\subsubsection{Dielectric properties of polymer composites}

A polymer composite is a composite that contains a polymeric material and at least one other material, organic or inorganic. The inorganic-polymer composite material will be discussed here as in ACPEL materials only inorganic materials such as $\mathrm{ZnS}, \mathrm{BaTiO}_{3}$ and $\mathrm{Ag}$ are blended with binder.

The advantage of integrating micro- or nanofillers into polymer based materials is that the properties associated with the inorganic phase and polymers can be coupled, leading to new functionality or new processing methods for inorganic materials [115]. Polymers are ease to produce and process and they have good adhesion with reinforcing elements and variety of substrates, not mentioning their light weight and ductile mechanical performances. Furthermore, as excellent electrical insulators, polymers exhibit high dielectric strength but low dielectric permittivity [116]. On the other hand, inorganic fillers are brittle materials, with high dielectric permittivity and thermal strength but usually have low dielectric strength [117]. Combining these two materials with compensating properties in a composite system could lead to a superior performance. The dielectric permittivity improvement in a polymer composite, which is also a heterogeneous system, is realized through the formation of so called interfacial polarization, termed the Maxwell-Wagner-Sillars effect (MWS effect) [118-120]. This is initiated by the differences in conductivities and permittivities of the inorganic and polymeric materials. As a result, space charges build up at the macroscopic interfaces. In contrast to the other types of polarization (atomic, electronic, dipolar), only this polarization changes the electric field, which are caused by the displacement or 
orientation of bound charge carriers.

Conventionally micro-sized inorganic materials are used to form polymer composites [121-123], which are named microcomposites. More recently, nanocomposites, which contain nanoparticles as the fillers, are preferred due to their superior dielectric properties. This advantage is due to the larger interfacial volume existing in nanocomposites compared to microcomposites [124], and the properties of the interfaces between the polymer and nanosized fillers are responsible for this improvement. Due to the interaction between polymer and fillers, the polymer morphology at the interface and local charge distribution at the nanoparticle surface may be changed. Moreover, the change of density and energy depth of trap sites due to the change in local structure at the interfaces affects the charge mobility and structure at the interfaces. Thus it is important to understand this interface.

\section{ZnS polymer composites}

As the main phosphor, the polymer composites containing $\mathrm{ZnS}$ phosphor microparticles are less studied, although extensive works have been carried out to study the dielectric enhancement of $\mathrm{ZnS}$ or $\mathrm{ZnS}$-capped nanoparticles [125-131]. Bessiere et al. studied the dielectric properties of sphalerite $\mathrm{ZnS}$ at solutions to understand the effects of different ions and its concentrations on the solid-solution interface [132]. A polyaniline-ZnS composite was prepared with different weight percentages and its temperature dependent DC conductivity was acquired, which followed a variable range hopping model [133]. The increase of $\mathrm{ZnS}$ concentration increased the conductivity but further increase reduced the DC conductivity. However, the polyaniline is a conducting polymer which does not apply to this case whereby polymeric binder is highly insulating. No other relevant literature could be found on the dielectric impedance spectroscopy study of $\mathrm{ZnS}$ polymer microcomposites. 


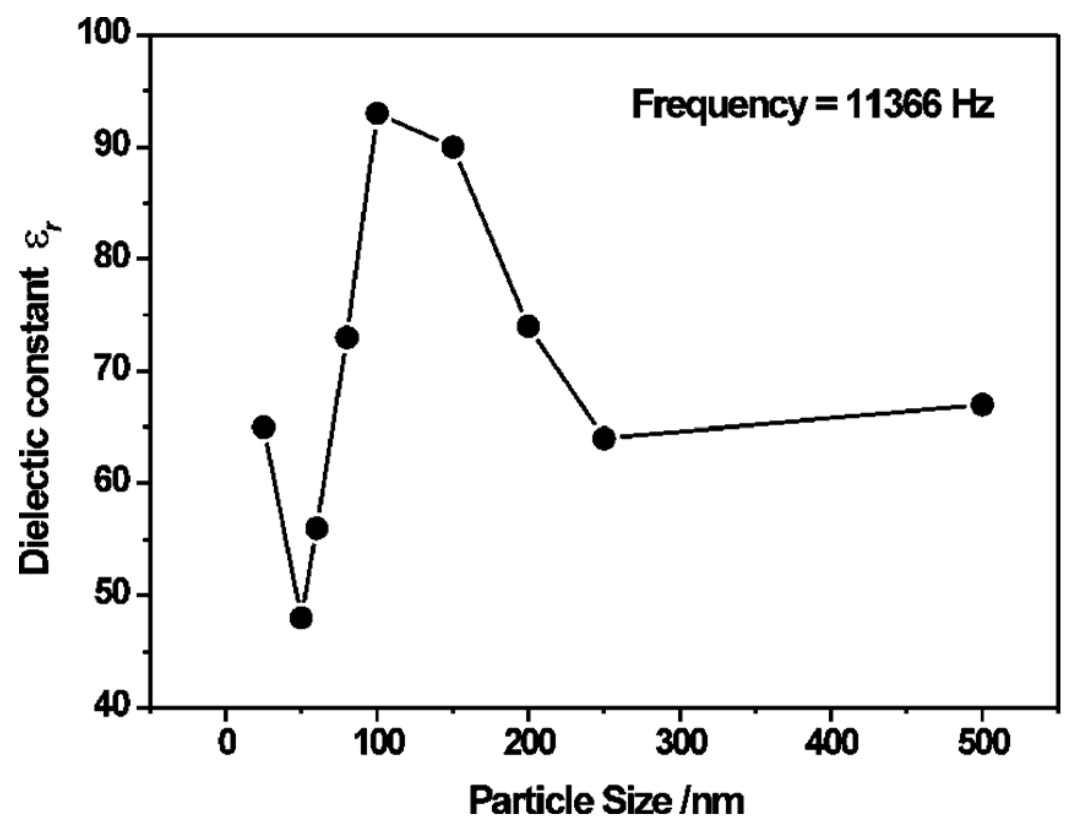

Figure 2.21: $\mathrm{BaTiO}_{3}$ particle size-dependence of the dielectric constants of the $\mathrm{BaTiO}_{3}$ /PVDF nanocomposites measured at room temperature at $1.14 \mathrm{kHz}$ [134].

\section{$\mathrm{BaTiO}_{3}$ polymer composites}

As a high k dielectric, $\mathrm{BaTiO}_{3}$ is extensively studied. $\mathrm{BaTiO}_{3}$ is a well-known fundamental ferroelectric perovskite oxide and is often used in multilayer ceramic capacitors due to high dielectric constant and low loss characteristics $[135,136] . \mathrm{BaTiO}_{3}$ experiences a phase transformation from paraelectric phase to ferroelectric phase at Curie temperature of around $130{ }^{\circ} \mathrm{C}$. This transformation enhances the dielectric properties and reduces the dielectric loss $[137,138]$. The size and concentration of the $\mathrm{BaTiO}_{3}$ nanoparticles affect the dielectric properties of the $\mathrm{BaTiO}_{3}$-polymer nanocomposites.

The size dependency of dielectric constant of a $\mathrm{BaTiO}_{3} / \mathrm{PVDF}$ nanocomposites is shown in Figure 2.21. The dielectric constant increases with the particle size first and decreases with sizes larger than $100 \mathrm{~nm}$. This agrees well with the findings of Fan et al. [139], where the dielectric constant is the largest with the sizes from $50-80 \mathrm{~nm}$. With the well fitting of the experimental results to the Jayasundere's relation, it is found that the decrease of the dielectric constant at larger size is due to the interactions between fillers and matrix [140]. 


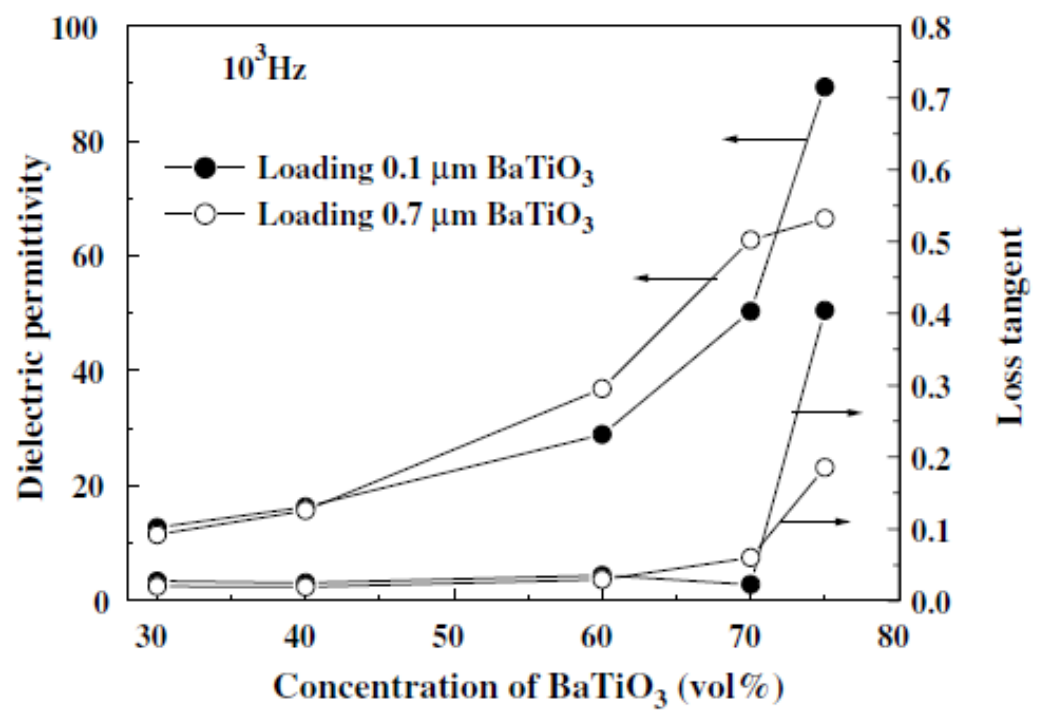

Figure 2.22: Dependence of dielectric properties of the composites on the volume concentration of $\mathrm{BaTiO}_{3}(0.1 \mu \mathrm{m}$ and $0.7 \mu \mathrm{m})$ at $10^{3} \mathrm{~Hz}$ measured at room temperature [141].

Similar trends were observed by other scientists as well [134, 141, 142].

In Figure 2.22, the increase of $\mathrm{BaTiO}_{3}$ concentration increases the dielectric permittivity and loss tangent for both the $0.1 \mu \mathrm{m}$ and $0.7 \mu \mathrm{m} \mathrm{BaTiO}_{3}$ at $10^{3} \mathrm{~Hz}$. Similarly, Fan et al. also observed the increase of dielectric permittivity with the concentration of $\mathrm{BaTiO}_{3}$ for the particle sizes of $30-50 \mathrm{~nm}$ and $50-80 \mathrm{~nm}$. Ramajo et al. ascribed this phenomenon to the increased porosity at high filler concentrations [143]. Mendes et al., however, related the increased real permittivity of higher $\mathrm{BaTiO}_{3}$ concentration to the increased amount of defective structures, which corresponded to the reduced degree of crystallinity of the polymer matrix [142].

The dielectric response study of $\mathrm{BaTiO}_{3}$ was conducted by various research groups [143-145]. Figure 2.23 shows the temperature and frequency dispersions of imaginary modulus for neat epoxy, composite-1 (40\% $100 \mathrm{~nm} \mathrm{BaTiO})_{3}$ and composite-2 (40\% 200 $\mathrm{nm} \mathrm{BaTiO}_{3}$ ). As indicated in Figure 2.23(a), $\alpha, \beta$ and $\gamma$ relaxation processes are observed in neat epoxy. However, when mixed with $\mathrm{BaTiO}_{3}$, only $\alpha$ relaxation and the MWS effect are significant in composite- 1 . With the size of $\mathrm{BaTiO}_{3}$ increased to $200 \mathrm{~nm}$, the $\alpha$ relaxation 
(a)

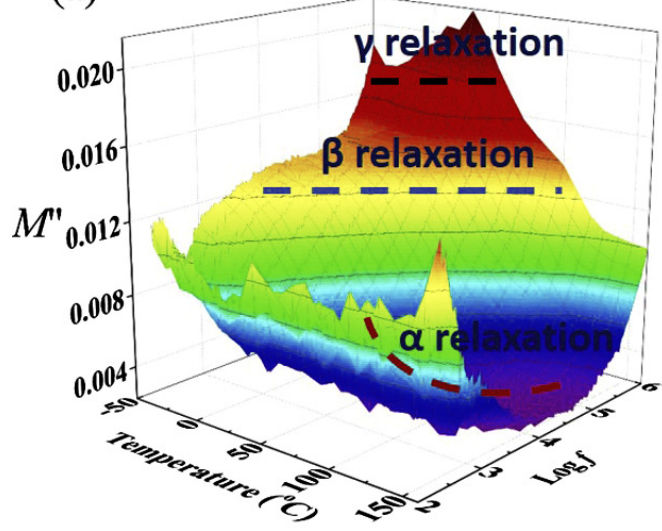

(c)

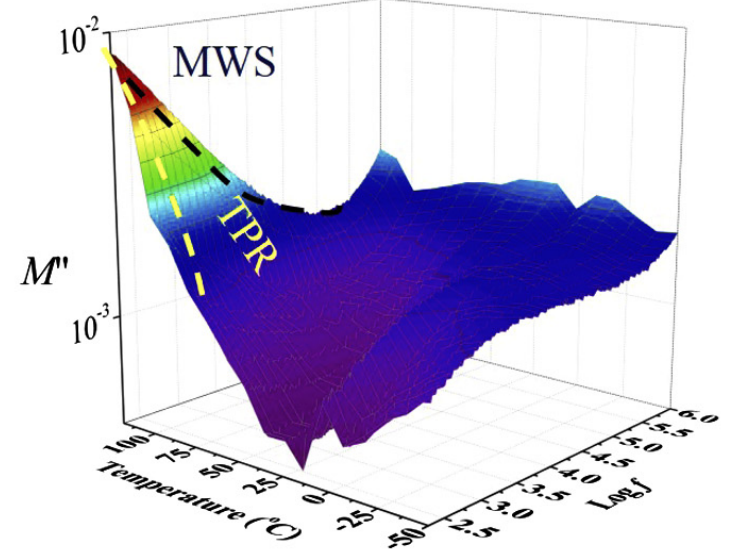

(b)

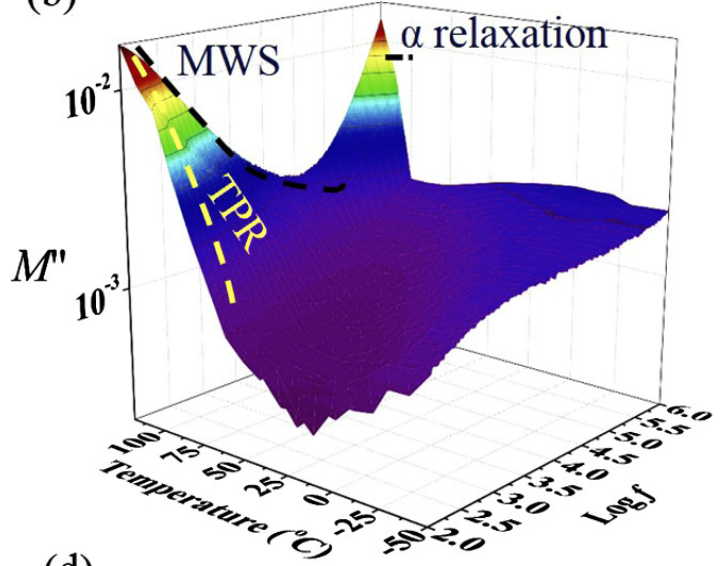

(d)

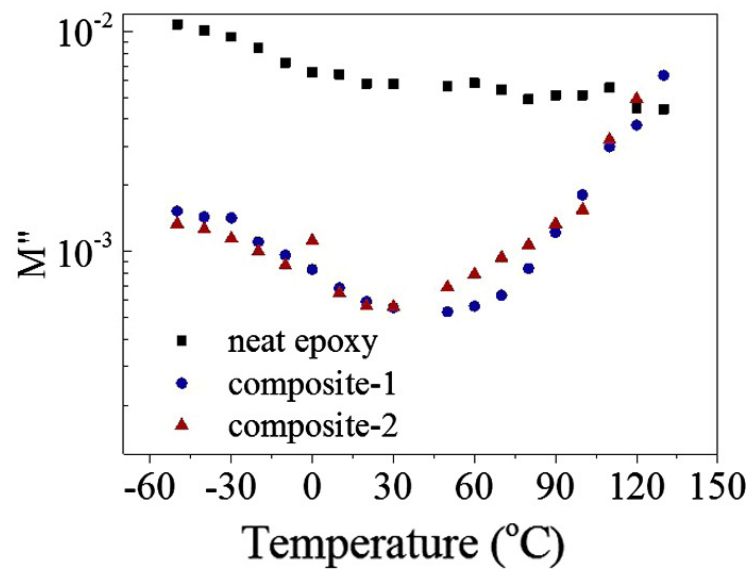

Figure 2.23: Imaginary part of electric modulus spectra as temperature and frequency for (a) neat epoxy, (b) composite-1 and (c) composite-2; (d) M" vs. temperature at $10^{3} \mathrm{~Hz}$ for all samples [144].

peak is largely suppressed. It is widely believed that mainly the MWS effect and tetragonal phase transition of $\mathrm{BaTiO}_{3}$ nanoparticles affect the dielectric response of the composites.

\section{Metal polymer composite}

Metal polymer composites are widely incorporated into bulk materials to enhance the dielectric permittivity of devices [146, 147]. At low concentration, the enhancement is achieved by the formation of the MWS effect at the metal/polymer composites [148]. At high volume concentrations of metal nanoparticles, this dielectric enhancement is then originated by the percolation theory [149]. When metal composites are used as electrodes, the 
porous structure and large surface area can facilitate high diffusion volume and large double layer capacitance [150]. Various equivalent circuit models of the double layer structure of the metal composite electrode are proposed by Cha et al. [151].

\subsection{Summary of research gaps}

In summary, although ACPEL devices were discovered years ago, the light excitation and emission mechanisms are still not well understood. There is also a research gap on the warm white colors of such devices. A number of printing techniques can be utilized to fabricate the hybrid warm white light source, whose color may be indicated by the colorimetric parameters, such as CIE xy color space, CCT and CRI. The impedance spectroscopy study of $\mathrm{ZnS}$ microcomposites is still lacked and the multilayered composites are less studied as well. 


\section{Chapter 3}

\section{PRINTED HYBRID EL FILMS BY DROP CASTING PROCESS}

\subsection{Introduction}

One of the limiting factors for the popularization of ACPEL devices is the limited color choices, with only blue, green and orange colors. The lack of red emitters will cause problems when producing white especially warm white ACPEL devices. Basically, there are three main approaches to achieve a warm white EL films: a white ZnS phosphor, mixing $\mathrm{ZnS}$ phosphors of different colors and using color conversion materials. Through the first approach, a white $\mathrm{ZnS}: \mathrm{Cu}, \mathrm{Cl}, \mathrm{Mn}$ phosphor consisting of only blue and yellow peaks was fabricated with the CRI of more than $85 \%$ [36]. Commercial white EL films are made by the mixed blue-green and yellow $\mathrm{ZnS}$ phosphors. No warm white EL films are achieved by the first two approaches due to lack of red phosphors. Although a red $\mathrm{ZnS}: \mathrm{Cu}, \mathrm{Cl}, \mathrm{Mn}, \mathrm{Te}$ phosphor powder was recently fabricated by Park et al. using a conventional wet synthesis and a sealed vessel method [37], the luminance of this red phosphor is only around $3.5 \mathrm{~cd} / \mathrm{m}^{2}$ at $200 \mathrm{~V}$ and $400 \mathrm{~Hz}$. This low luminance is not sufficient in producing warm white EL films. Hence, various researches had worked on the third approach of implementing downshifting materials, both organic dyes and inorganic phosphors. Downshifting is a color conversion process which converts a high-energy to low-energy photon [1]. In particular, Kim used two small molecules (coumarin-6 and DCJTB) to convert blue emission from $\mathrm{ZnS}$ phosphors to green and red emissions and obtain a CIE of $(0.46,0.46)$. These two organic dyes were inserted as separate layers between a transparent electrode and $\mathrm{ZnS}$ phosphor layers. Another experiment conducted by Park et al. demonstrated the incorporation of yellow $\mathrm{Tb}_{3} \mathrm{Al}_{5} \mathrm{O}_{12}: \mathrm{Ce}^{3+}$ and red $\mathrm{CaS}: \mathrm{Eu}^{2+}$ phosphors over the transparent substrate to produce a white light with a CRI of 89 and a luminance efficacy of $3.32 \mathrm{~lm} / \mathrm{W}$. However, 
$\mathrm{Eu}$ is not favored as it is a rare earth and toxic material. In both studies, no warm white color is reported.

In this study, a big molecular dye F8BT acting as the green converter and a small molecular dye DCJTB as the red converter were used. F8BT and DCJTB were used as they were demonstrated as stable in organic solar cells and OLED [152, 153]. As a preliminary experiment, a simple drop casting process was utilized to demonstrate the downshifting capabilities of both organic dyes. To further simplify the fabrication process, both dyes were coated together in one layer. Different operating voltages and frequencies were applied to facilitate the color tuning. Various colors were obtained with altering the mass ratio and concentration of F8BT and DCJTB mixed solutions.

\subsection{Experimental methods}

\subsubsection{Fabrication of forward and reversed EL films}

Forward and reversed blue/green EL films were fabricated by the low-cost screen-printing process using a Micro-tec MT-850 screen printer. The printing process for forward devices is illustrated in Figure 3.1. The substrates used for the screen-printing process were PET pre-coated with ITO $(\sim 100 \Omega /$ sq, Nejilock) with the thickness of $200 \mu \mathrm{m}$. Blue (Gwent C2061027P15) or green (Gwent C2070209P5)) ZnS phosphor powders ( 20 $\mu \mathrm{m}$ in diameter) was premixed with a polymeric binder (Gwent R2070613P2) consisting of a polyurethane film former and epoxy resin. A $105 \mathrm{~mm} \times 105 \mathrm{~mm}$ square emitting layer was formed with the phosphor paste printed on top of the ITO-coated PET using the printing speed of $300 \mathrm{~mm} / \mathrm{s}$. Thereafter, the devices were placed in Tabai oven and cured at 130 ${ }^{\circ} \mathrm{C}$ for $30 \mathrm{~min}$. After the emitting layer was fully cured, a $105 \mathrm{~mm} \times 105 \mathrm{~mm}$ dielectric layer ( $\sim 35 \mu m$ thick) was printed over it using high-k dielectric material barium titanate $\left(\mathrm{BaTiO}_{3}\right)$ nanoparticles $(\sim 100 \mathrm{~nm}$, Gwent D2070209P6) dispersed in binder with the print-

ing speed of $300 \mathrm{~mm} / \mathrm{s}$. A second curing step was followed at $130{ }^{\circ} \mathrm{C}$ for $30 \mathrm{~min}$. Finally, Ag nanoparticle paste (Gwent C2131014D3) was printed as the back electrode $(\sim 10 \mu \mathrm{m}$ 


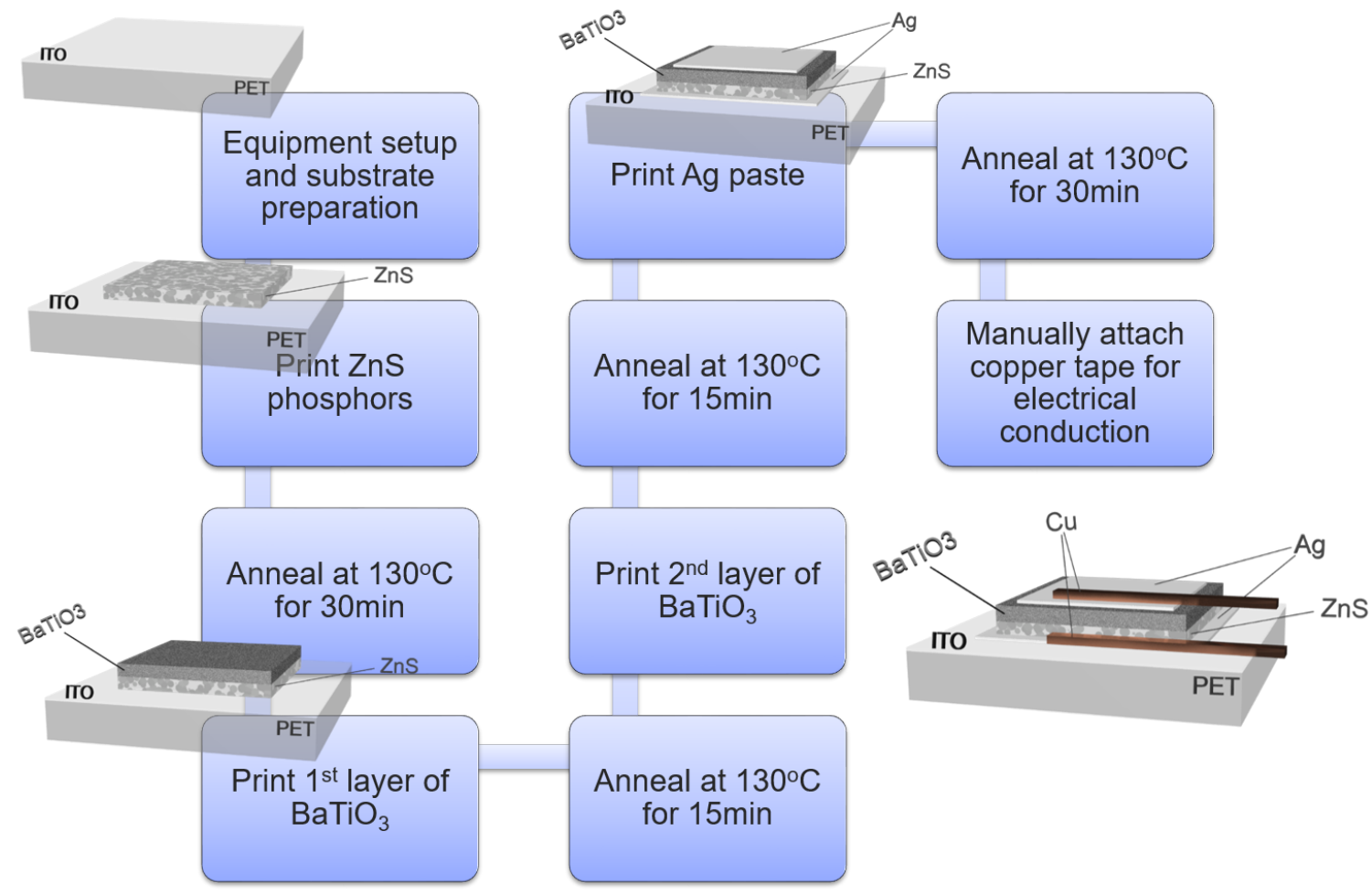

Figure 3.1: The screen printing process flow for forward ACPEL devices. Device architectures along the process are presented as the insets with the final device structure presented.

thick) using the printing speed of $30 \mathrm{~mm} / \mathrm{s}$ and the final pattern size formed was $100 \mathrm{~mm}$ $\times 100 \mathrm{~mm}$. For the external electrical supply, two copper stripes were attached to the two electrodes and EL was emitted from the PET side for the forward EL films. The final device structure for forward devices is shown in the inset of Figure 3.1. For the reversed EL films, the dielectric layer was printed first followed by the emitting layer with the same printing speed and pattern size as the forward devices, as illustrated in Figure 3.2. A layer of PEDOT:PSS (CLEVIOS S V3 HV) with the size of $100 \mathrm{~mm} \times 100 \mathrm{~mm}$ was printed prior to a Ag layer using the printing speed of $100 \mathrm{~mm} / \mathrm{s}$. The size of the Ag layer was only $10 \mathrm{~mm} \times 100 \mathrm{~mm}$ and its function was to stabilize charges over the PEDOT:PSS electrode. The reversed EL film emits lights from the PEDOT:PSS side. The final device structure for reversed EL films is shown in the inset of Figure 3.2. 


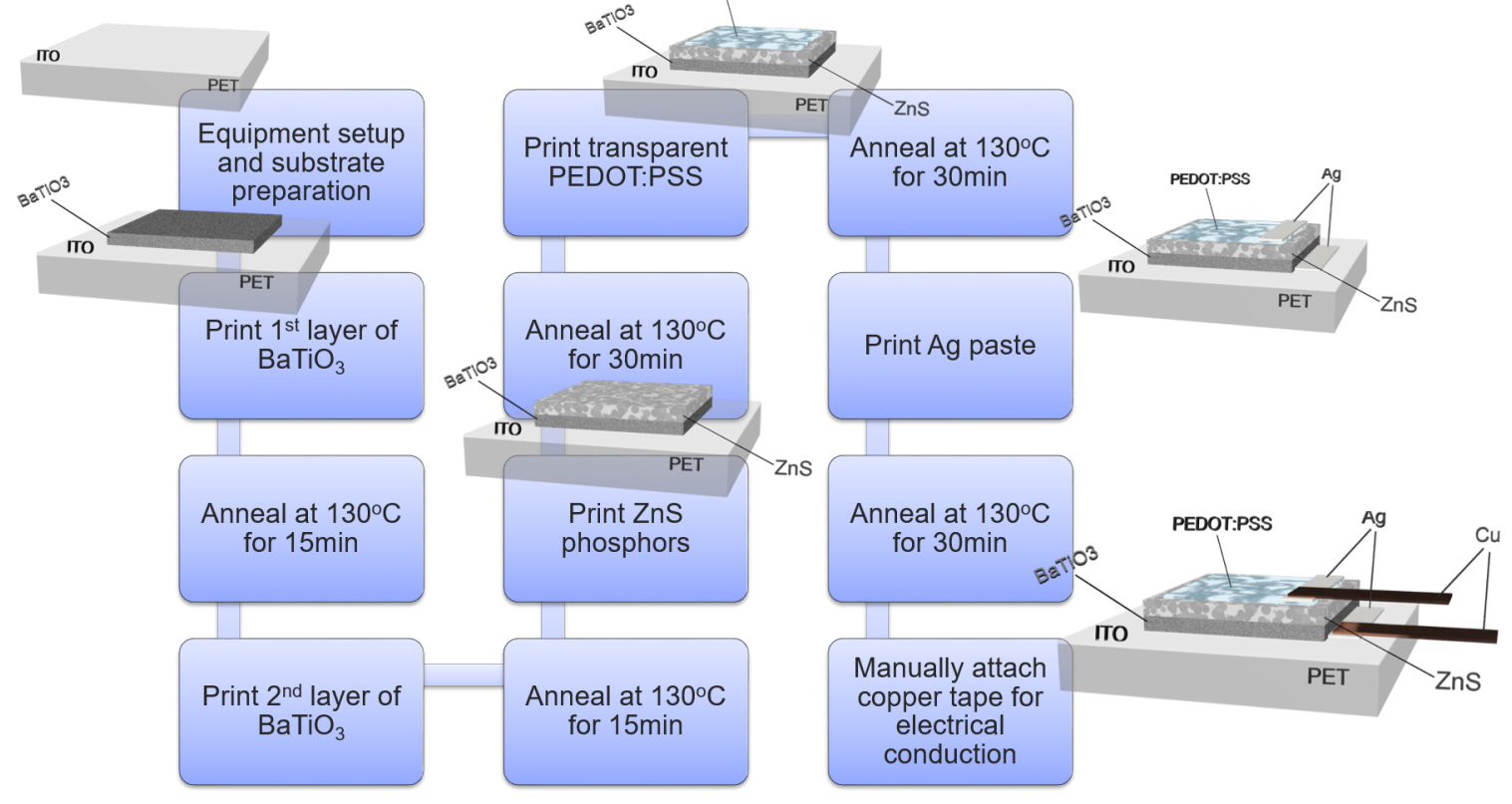

Figure 3.2: The screen printing process flow for reversed ACPEL devices. Device architectures along the process are presented as the insets with the final device structure presented.

\subsubsection{Selection of dye, solvent and coating method}

F8BT (Lumtec LT-S957) and DCJTB (Lumtec LT-E704) were used as the downshifting organic dyes with the molecular structures as shown in Figure 3.3. These two materials were primarily dissolved in chloroform $\left(\mathrm{CHCl}_{3}, 99 \%\right.$ Sigma-Aldrich). In order to explore the possibility for large-scale processing, a $2.0 \mathrm{mg} / \mathrm{mL}$ dye in $\mathrm{CHCl}_{3}$ solution was mixed with binder or polydimethylsiloxane (PDMS) and the resulted dye-binder mixture was cured at $130^{\circ}$ for $30 \mathrm{~min}$ while the dye-PDMS mixture was cured at $70{ }^{\circ} \mathrm{C}$ for $10 \mathrm{~min}$. Their PL emission spectra were obtained. With the unsatisfactory results from the mixed binder or PDMS results which will be shown in the results section, only the dye in $\mathrm{CHCl}_{3}$ solution was suitable and spin coating process was primarily considered for the coating method using a Laurell WS-400-6NPP-LITE spin coater. A layer of the F8BT and DCJTB mixed solution was spin-coated on both forward and reversed EL films. Prior to the coating process, forward devices were cleaned by isopropanol (IPA) (Sigma-Aldrich). After several trials of spin coating, the lowest coating speed of 300 revolutions per minute (RPM) and 


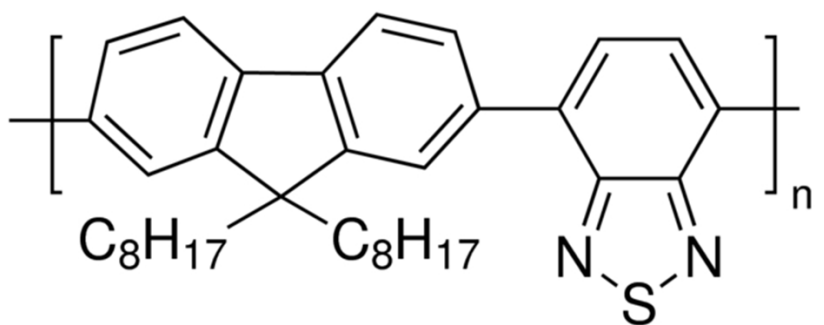

(a)

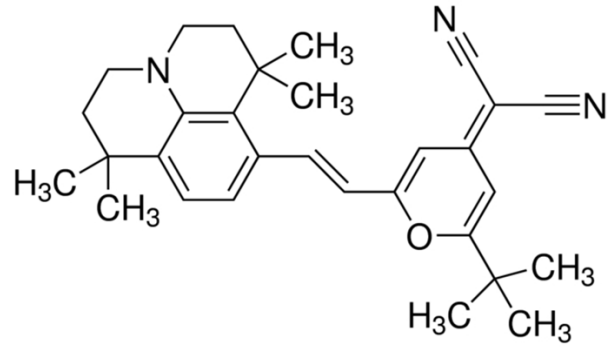

(b)

Figure 3.3: Chemical structures of (a) F8BT and (b) DCJTB.

lowest acceleration speed of 116 RPM were selected. With the total dye concentration fixed at $0.5 \mathrm{mg} / \mathrm{mL}$, the mass ratio between DCJTB and F8BT was varied to be 1:1, 1:2, 1:3, 1:4, 1:7, 1:9, 2:1, 3:1, 4:1, 7:1, and 9:1. These different solutions were spin-coated at room temperature and the resulted coating was also dried at room temperature.

\subsubsection{Drop casting of dye-chloroform solution}

Spin coating was not an appropriate method to coat F8BT and DCJTB organic dyes. As an alternative, drop casting method is more suitable due to its simplicity and material saving. F8BT and DCJTB were first coated individually onto the forward and reversed EL films to study the downshifting capability of each organic dye. Figure 3.4 shows the process flow for the drop casting method. To drop cast a layer of F8BT dyes, $15.6 \mathrm{mg}$ of F8BT was dissolved in $4 \mathrm{~mL}$ of $\mathrm{CHCl}_{3}$ at room temperature. Thereafter, $20 \mu \mathrm{L}$ of the above $3.9 \mathrm{mg} / \mathrm{mL}$ F8BT solution was dropped over the horizontally flat PET surface of forward EL films by a pipette which was then placed $2 \mathrm{~cm}$ over the sample. After drying at room temperature, the forward hybrid EL films exhibit the final device structure as shown in Figure 3.5(a). The typical diameter of the drop casted organic dye is around $1 \mathrm{~cm}$. As for the reversed hybrid devices, $20 \mu \mathrm{L}$ of the above F8BT solution was drop casted over the PEDOT:PSS side of the reversed EL films and the hybrid device structure is illustrated in Figure 3.5(b). The forward and reversed hybrid EL films incorporating DCJTB were fabricated with the same 

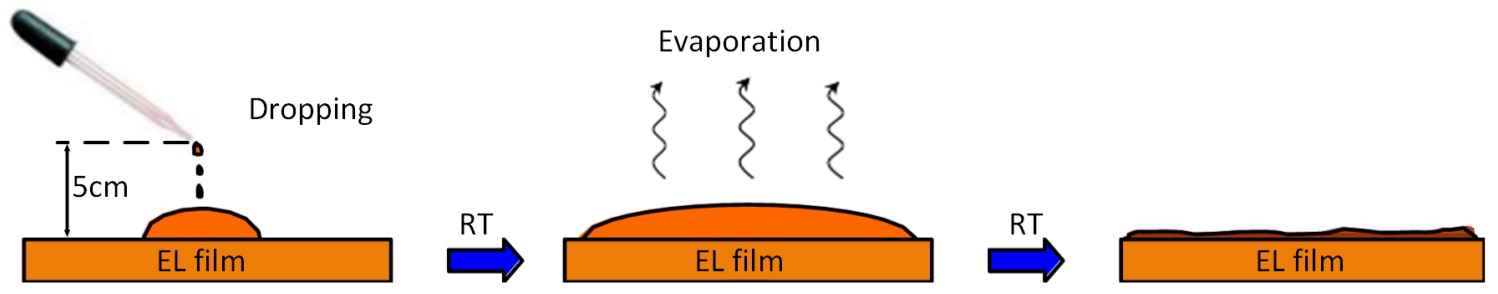

Figure 3.4: The drop casting process. RT: room temperature.

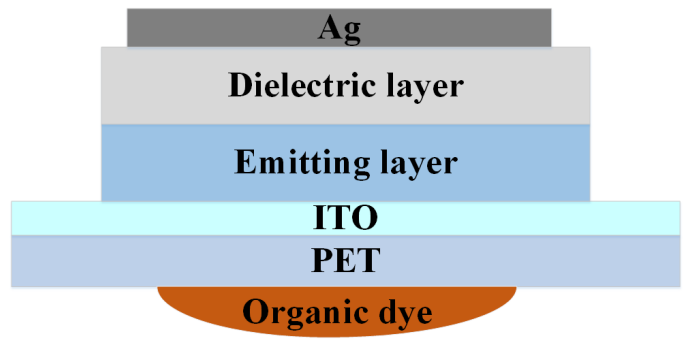

(a)

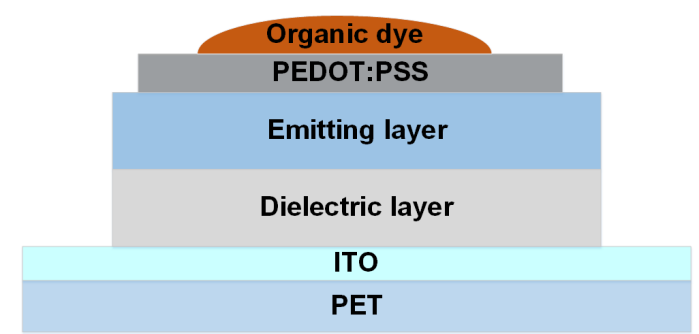

(b)

Figure 3.5: Structures of (a) forward and (b) reversed hybrid ACPEL devices with a layer of drop casted organic dye.

method using a $1.9 \mathrm{mg} / \mathrm{mL}$ DCJTB solution prepared by dissolving $11.4 \mathrm{mg}$ of DCJTB in $6 \mathrm{~mL}$ of $\mathrm{CHCl}_{3}$ at room temperature.

\subsubsection{Fabrication of white hybrid EL films}

In order to tune the emission colors of the hybrid EL films, a mixed F8BT- and DCJTB$\mathrm{CHCl}_{3}$ solution was drop casted over reversed devices using the same process stated in section 3.2.3. The mixture was obtained by dissolving F8BT and DCJTB separately into $\mathrm{CHCl}_{3}$ first and mixing the resulting solutions. Two sets of experiments were conducted in the present study. The first one involved various F8BT:DCJTB mass ratios. 18mg of F8BT was added into $4.5 \mathrm{~mL}$ of $\mathrm{CHCl}_{3}$ to prepare a starting solution. By adding $1,1.286,1.8$, 2.25 , and $3 \mathrm{~mL}$ of the $2 \mathrm{mg} / \mathrm{mL} \mathrm{DCJTB-CHCl}{ }_{3}$ solution, the F8BT:DCJTB mass ratios of 9:1, 7:1, 5:1, 4:1, and 3:1 were obtained and the respective concentrations of F8BT-DCJTB solution (final concentration) were $3.64,3.56,3.43,3.33$ and $3.2 \mathrm{mg} / \mathrm{mL}$ respectively. The final concentration was calculated by dividing the total mass of F8BT and DCJTB by the 
Table 3.1: Variation of concentration and mass ratio of the dye mixture used for the fabrication of white hybrid ACPEL devices.

\begin{tabular}{lcc}
\hline Experiment set & Final concentration $(\mathrm{mg} / \mathrm{mL})$ & Mass ratio \\
\hline 1-mass ratio & $3.64,3.56,3.43,3.33,3.20$ & $9: 1,7: 1,5: 1,4: 1,3: 1$ \\
2-final concentration & $2.40,1.92,1.60,1.37,1.20$ & $3: 1$ \\
\hline
\end{tabular}

total volume. The second set of experiment involved diluting the final mixture with the F8BT:DCJTB mass ratio of 3:1 from the first experiment to achieve different final concentrations. By adding $2.5 \mathrm{~mL}$ of $\mathrm{CHCl}_{3}$ successively to the previous mixture up to 5 times, the final concentrations of $2.40,1.92,1.60,1.37$ and $1.20 \mathrm{mg} / \mathrm{mL}$ were obtained. As summarized in Table 3.1, $20 \mu \mathrm{L}$ of the mixtures in these two sets of experiments were drop casted over reversed EL films to fabricate hybrid devices.

\subsubsection{Characterization methods}

Photoluminescence (PL) emission and excitation spectra were acquired using a Fluorolog3 PL system. The absorbance and transmittance spectra were measured by a Shimadzu UV-2450 UV-VIS spectrometer from $350 \mathrm{~nm}$ to $800 \mathrm{~nm}$. The $\mathrm{ZnS}$ phosphor powders, $\mathrm{BaTiO}_{3}$ powders and EL films were examined under Ultra Plus FE-scanning electron microscope (SEM) from ZEISS and using Energy-dispersive X-ray spectroscopy (EDX). The dark field images of operating EL films were taken using an Olympus upright metallurgical microscope BX53M by exciting the devices at $110 \mathrm{~V}$ and $400 \mathrm{~Hz}$ with the characterization light turned off under the dark field mode. EL spectra, luminance, and CIE were measured using the SpectraWin software of the PR655 spectroradiometer from Photo Research. The measurement was performed at a $2^{\circ}$ observer angle with a spot size of about $5 \mathrm{~mm}$ in a dark enclosure at ambient conditions. The power luminous efficacy was obtained by dividing the luminous flux by the electrical power. The luminous flux was measured using 


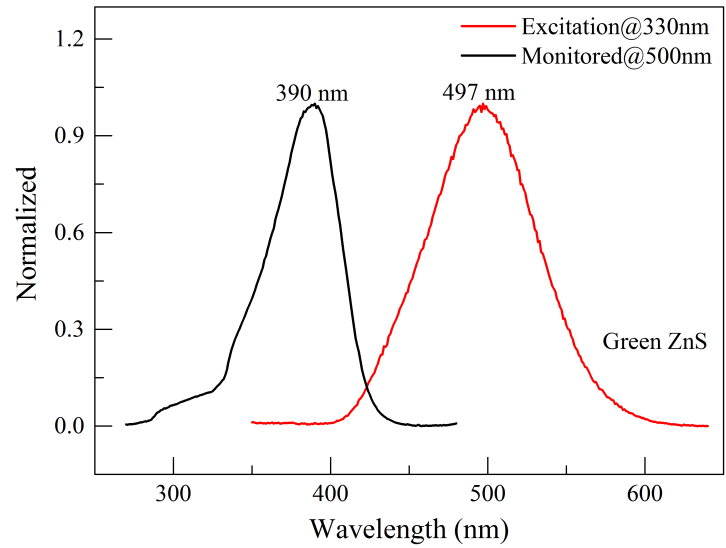

(a)

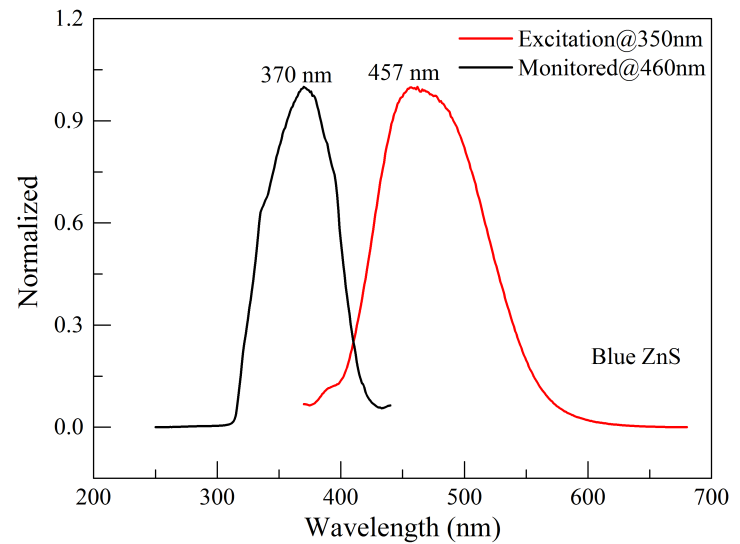

(b)

Figure 3.6: PL emission and excitation spectra of (a) green and (b) blue $\mathrm{ZnS}$ phosphors.

a halfmoon integrating sphere from Labsphere and the electrical power was measured by a YOKOGAWA WT210 digital power meter. The AC sinusoidal excitation voltages were applied by a Pacific 105-AMX power source without any DC bias voltage. EL and white light aging were performed to study the durability of reversed blue and F8BT and DCJTB in hybrid devices. In order to reduce experimental errors, six un-passivated samples dropcasted with the F8BT:DCJTB mass ratio of 9:1 were tested for each aging study. EL aging was carried out by operating the devices continuously at $110 \mathrm{~V}$ and $400 \mathrm{~Hz}$ in a dark room at $25{ }^{\circ} \mathrm{C}$ and $70 \%$ relative humidity. For white light aging, a fluorescent white light box with the radiance power of $4.3 \mathrm{Wsr}^{-1} \mathrm{~m}^{-2}$ was used to age the hybrid EL films at $25^{\circ} \mathrm{C}$ and $70 \%$ relative humidity in a dark room. The hybrid EL film with the F8BT:DCJTB mass ratio of 9:1 and final concentration of $1.6 \mathrm{mg} / \mathrm{mL}$ was placed directly over the white light box with the dye layer facing the light source and AC voltage was not applied to the device during white light aging. 


\subsection{Results and discussion}

\subsubsection{Forward and reversed EL films}

The PL emission and excitation spectra of green and blue $\mathrm{ZnS}$ phosphor powders are shown in Figure 3.6. In Figure 3.6(a), green $\mathrm{ZnS}$ phosphors exhibit emissions with the peak wavelength of $497 \mathrm{~nm}$ when excited by $330 \mathrm{~nm}$ UV light. The full width at half maximum (FWHM) is $82 \mathrm{~nm}$. The excitation spectrum of a $500 \mathrm{~nm}$ emission from green phosphors is at its maximum at $390 \mathrm{~nm}$. The emission peak of blue $\mathrm{ZnS}$ phosphors is at $457 \mathrm{~nm}$ when excited by $350 \mathrm{~nm}$ UV light as indicated in Figure 3.6(b). The PL excitation spectrum tells us that blue $\mathrm{ZnS}$ phosphors are most efficiently excited by $370 \mathrm{~nm}$ photons. As shown in Figure 3.7(a), ZnS phosphor powders have irregular shapes with the average size of around $20 \mu \mathrm{m}$. Figure 3.7(b) reveals the cross-section of $\mathrm{ZnS}$ phosphors. A layer of coating with the thickness of submicron is seen for each $\mathrm{ZnS}$ powder. The EDX result tells us that this coating is $\mathrm{Al}_{2} \mathrm{O}_{3}$. The $\mathrm{BaTiO}_{3}$ dielectric powders used for insulating purposes in EL films are in nanometer size and also with irregular shapes as shown in Figure 3.7(c).

The SEM images of forward and reversed EL films are shown in Figure 3.8. The main difference between these two types of devices is the position of the $\mathrm{ZnS}$ phosphor layer. Especially in reversed EL films, there is no clear indication of visible transparent layer, which is PEDOT:PSS. This shows that the thickness of PEDOT:PSS is very small. When excited by an $\mathrm{AC}$ electric signal with the voltage of $110 \mathrm{~V}$ and frequency of $400 \mathrm{~Hz}$, the forward EL films with green $\mathrm{ZnS}$ phosphors (forward green) exhibit green emission with the peak wavelength of $504 \mathrm{~nm}$ and FWHM of $76 \mathrm{~nm}$. As seen in Figure 3.9(a), in spite of having different peak intensities, the reversed EL films with green $\mathrm{ZnS}$ phosphors (reversed green) emit EL lights with the same peak wavelength and FWHM as forward green. Both forward and reversed devices with blue $\mathrm{ZnS}$ phosphors (forward Blue, reversed Blue) give out EL with the same peak wavelength of $456 \mathrm{~nm}$ and FWHM of $100 \mathrm{~nm}$. In Figure 3.9(b), it is observed that CIE for all samples shift towards blue region as the operating 


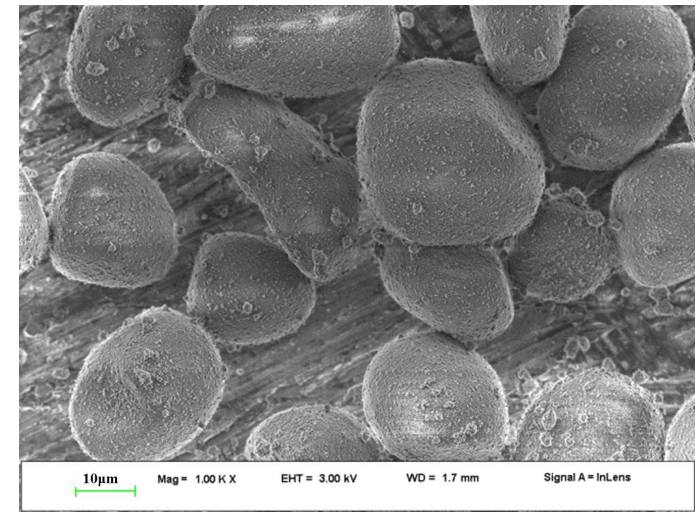

(a)

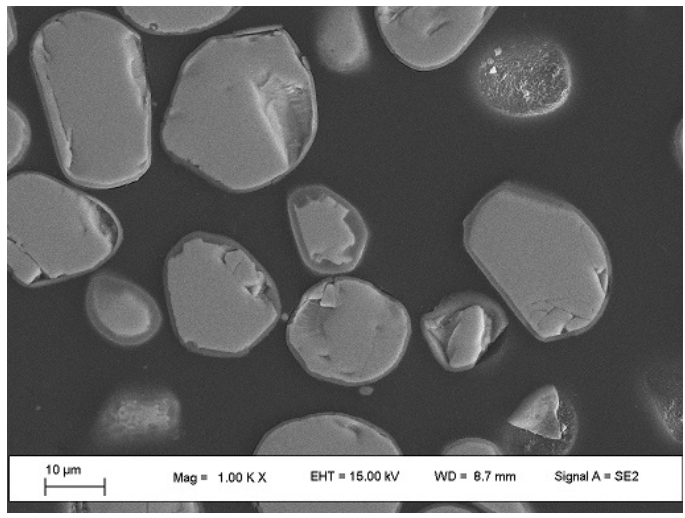

(b)

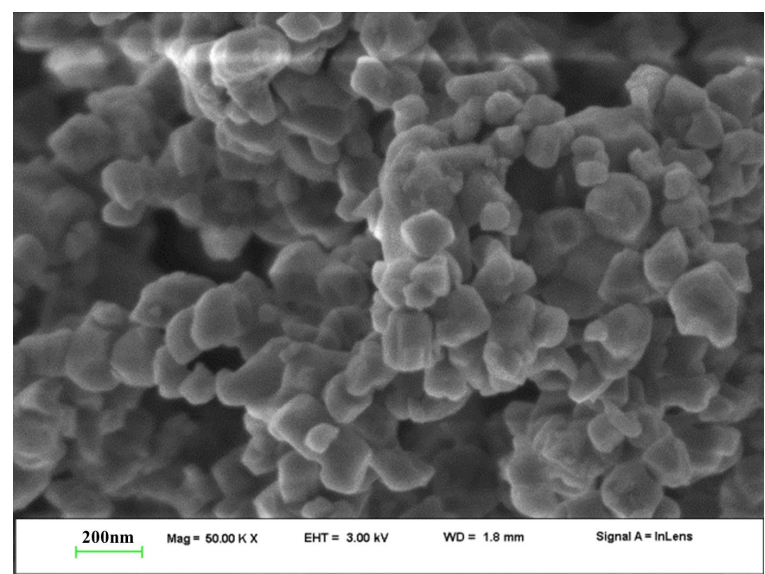

(c)

Figure 3.7: SEM images of (a) $\mathrm{ZnS}$ phosphor and (b) $\mathrm{BaTiO}_{3}$ dielectric powders.

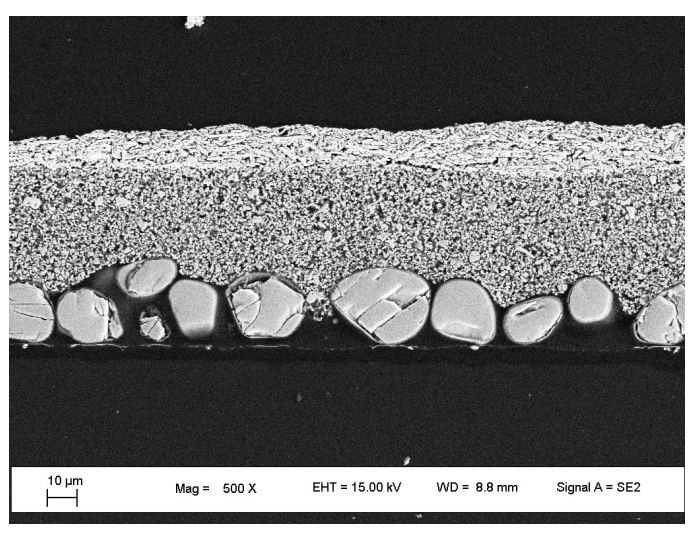

(a)

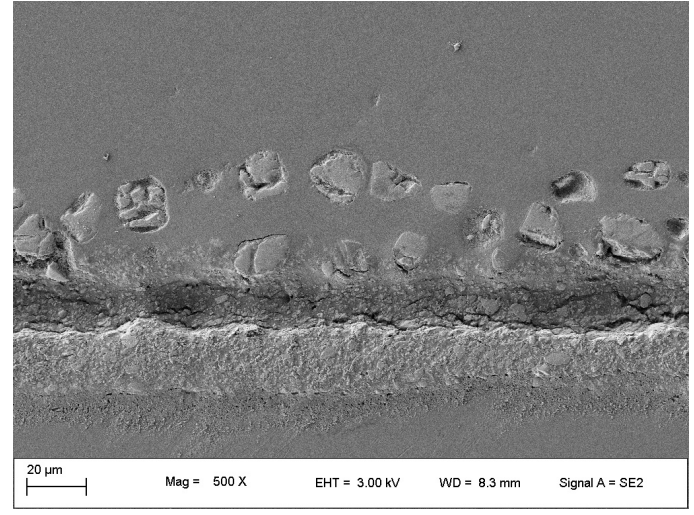

(b)

Figure 3.8: SEM images of (a) forward and (b) reversed EL films. 


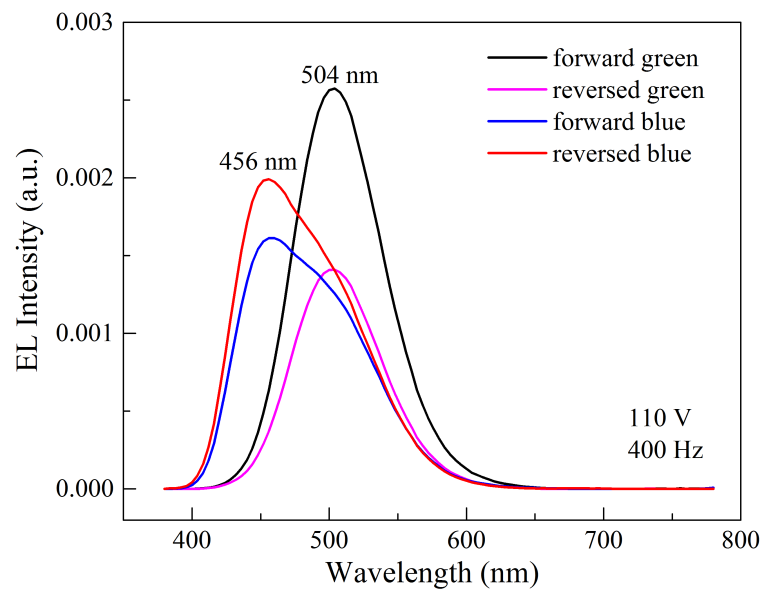

(a)

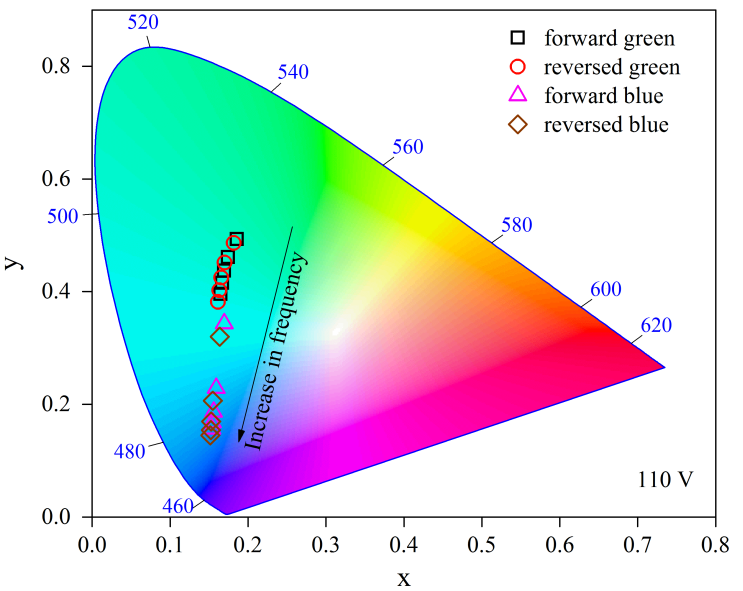

(b)

Figure 3.9: (a) EL spectra at $110 \mathrm{~V}$ and $400 \mathrm{~Hz}$ and (b) CIE at $110 \mathrm{~V}$ and different frequencies for Forward Green, Reversed Green, Forward Blue and Reversed Blue EL films.

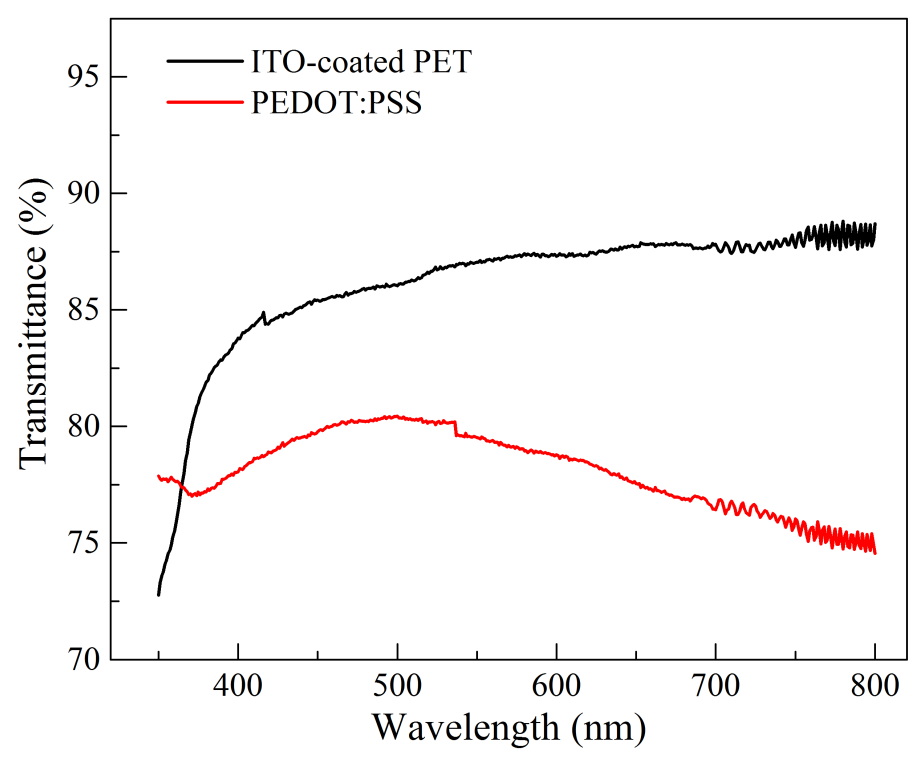

Figure 3.10: Optical transmittance of ITO-coated PET and PEDOT:PSS.

frequency increases. This is due to the frequency-dependent charge transport and relaxation behaviors which will be elaborated in details in Chapter 5. The reversed devices are more blueish in color than the forward ones. This is due to the higher transmittance of shorter wavelength photons than longer wavelength photons of the PEDOT:PSS layer as indicated by the transmittance spectra in Figure 3.10.

In Figure 3.11, the luminance of forward green, reversed green, forward blue and re- 


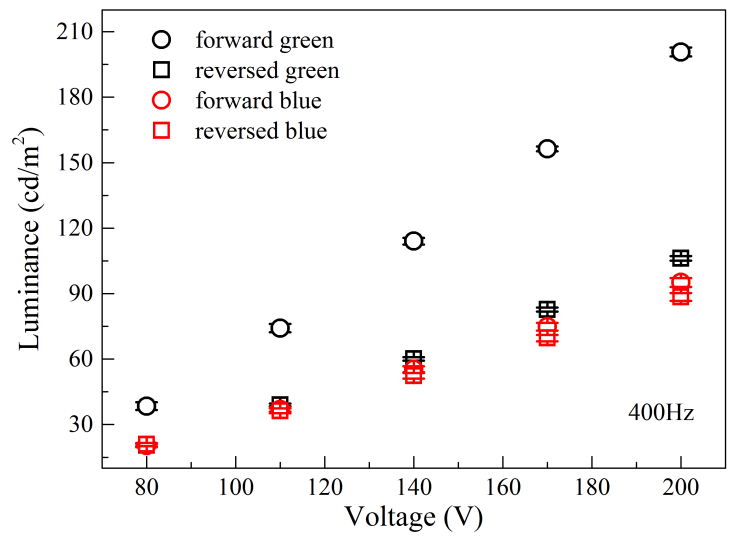

(a)

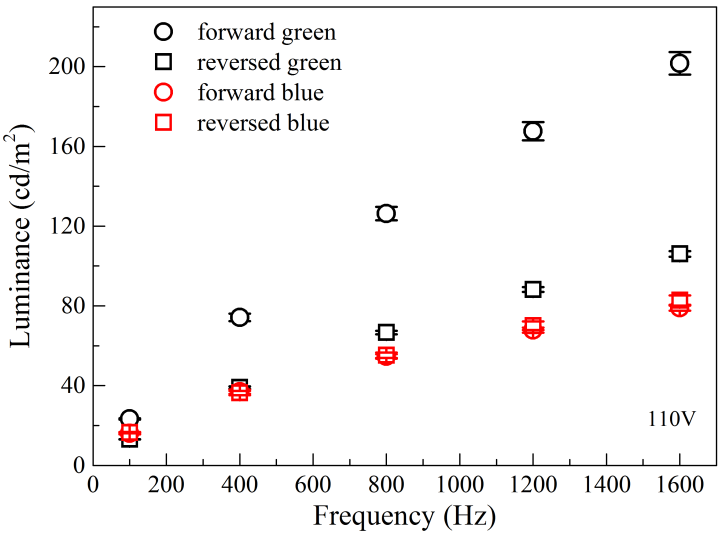

(b)

Figure 3.11: Luminance as a function of (a) voltage at $400 \mathrm{~Hz}$ and (b) frequency at $110 \mathrm{~V}$ for forward and reversed blue or green EL films.

versed blue as a function of voltage and frequency is shown. For all the four types of EL films, luminance increases with voltage or frequency. However, there is a tendency for the luminance to saturate at higher frequency. This agrees well with the observation by Stanley et al. [92]. For both forward and reversed structures, the luminance of green EL films is higher than that of blue ones. Moreover, the forward devices are brighter than the reversed ones in either green or blue phosphors. However, it is noticed that the luminance margin between forward and reversed devices is a lot smaller in blue phosphors than in green phosphors. The measured luminous efficacy values of forward green, forward blue, reversed green and reversed blue EL films are 3.51, 2.29, 3.65, and $1.74 \mathrm{~lm} / \mathrm{W}$. The power efficacy of green devices is higher than that of blue devices. However, the overall luminous efficacy is in a order much smaller than that of $128 \mathrm{~lm} / \mathrm{W}$ in the GaN LED [154]. Therefore, purely in the view of emission intensity and power efficacy, forward green should be used for color tuning. However, if the performances of forward and blue hybrid devices are to be compared, blue devices should be used. In our case, these four devices will be tested and the selection criteria is based on the color output and film quality. 


\subsubsection{Solvent effect and spin coating method}

$\mathrm{CHCl}_{3}$ was used to dissolve F8BT and DCJTB powders. Their PL emission and absorbance spectra are shown in Figure 3.12. When excited by $350 \mathrm{~nm} \mathrm{UV}$ light, the $\mathrm{F} 8 \mathrm{BT}-\mathrm{CHCl}_{3}$ solution exhibits a green emission with a peak wavelength of $548 \mathrm{~nm}$ and a FWHM of 67 $\mathrm{nm}$ while the F8BT-PET film experiences a slight blue shift to a peak wavelength of 543 $\mathrm{nm}$. With the peak wavelengths at $468 \mathrm{~nm}$ and $478 \mathrm{~nm}$, the absorbance spectra of the F8BT$\mathrm{CHCl}_{3}$ solution and F8BT-PET film fall within the EL spectra of the forward and reversed ACPEL devices as shown in Figure 3.9(a). This demonstrates the downshifting capability of F8BT and this green dye is more effectively in absorbing blue photons. The increased absorbance at wavelengths less than $350 \mathrm{~nm}$ is due to the absorption in ITO. On the other hand, the DCJTB-CHCl 3 solution exhibits a red emission with the peak wavelength of 615 $\mathrm{nm}$ and FWHM of $54 \mathrm{~nm}$. The DCJTB layer on a PET film experiences a red shift of the PL emission spectrum to the peak wavelength of $630 \mathrm{~nm}$. From the absorbance spectra in Figure 3.12(b), both the DCJTB-CHCl ${ }_{3}$ solution and DCJTB-PET film are able to absorb a broad spectrum of blue to green lights. However, since the absorbance peak wavelength is at $510 \mathrm{~nm}$ for both samples, DCJTB is better at absorbing the green emissions from F8BT as shown in Figure 3.12(a).

Polymeric binder and PDMS are considered to mix with the dye- $\mathrm{CHCl}_{3}$ solution, because binder is proved in screen printing technology and PDMS has been used extensively in spin coating process. Figure 3.13 shows the PL emission spectra of F8BT and DCJTB mixed in binder and PDMS before and after curing process. The photo-excited F8BTbinder solution emits green light with the peak wavelength of $545 \mathrm{~nm}$ but experiences a suppression of the green peak after curing. There is another peak at $385 \mathrm{~nm}$ for the cured sample. This suppression effect is even more significant for the F8BT-PDMS solution before and after curing. DCJTB mixed in binder or PDMS, however, does not suffers from the intensity suppression but a blue shift from $595 \mathrm{~nm}$ to $550 \mathrm{~nm}$ for the DCJTB-PDMS mixture. Interestingly, the peak wavelength of DCJTB-binder shifts from $595 \mathrm{~nm}$ to $636 \mathrm{~nm}$ 


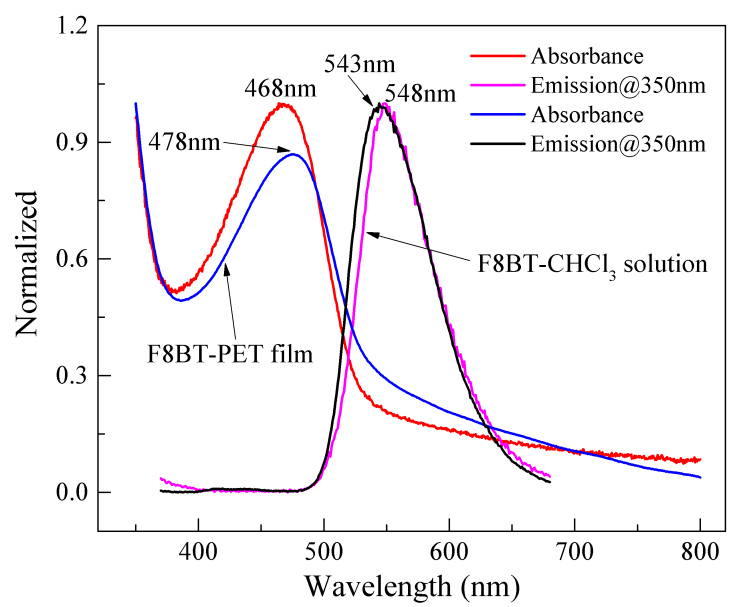

(a)

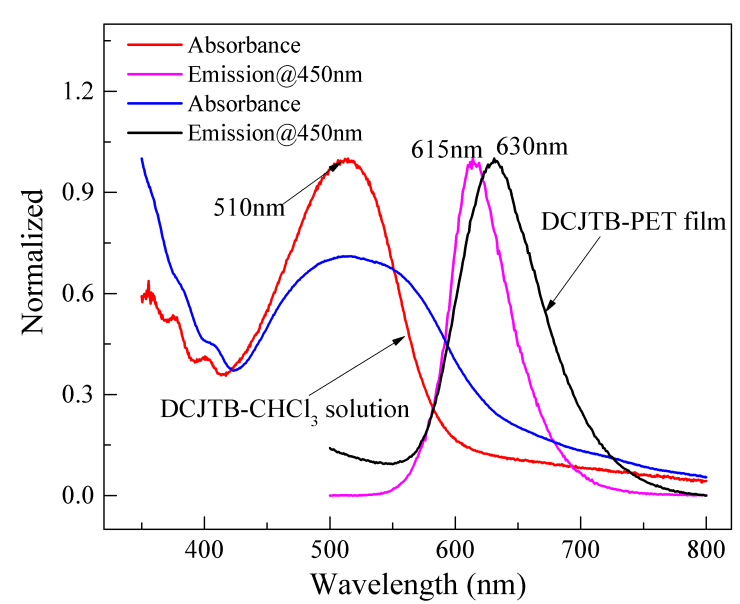

(b)

Figure 3.12: PL emission and absorbance spectra of (a) F8BT and (b) DCJTB in $\mathrm{CHCl}_{3}$ and on PET films.

after curing. This may be due to the different energy transfer mechanism between DCJTBbinder and DCJTB-PDMS. In the consideration of the low green peak intensity for cured F8BT-PDMS and a large blue shift of the cured DCJTB-PDMS, PDMS is not suitable for the coating of these two organic dyes. Binder solution is also discarded as the green emission is largely quenched. As such, only dye- $\mathrm{CHCl}_{3}$ solution was spin-coated onto EL films for the demonstration of downshifting capabilities. However, the coating finish is not uniform. Even with the lowest speed, the spin-coated dye layer cannot be distributed evenly over the sample surface. This poor surface finish is caused by the high evaporation speed of $\mathrm{CHCl}_{3}$. Moreover, as shown in Figure 3.14, there is no noticeable green emission even with the mass ratio of F8BT and DCJTB at 9:1 due to the low solubility of F8BT in $\mathrm{CHCl}_{3}$. Therefore, the spin coating methodology is still not suitable for the coating of organic dye layer onto EL films. Considering the simplicity and conservation of materials, drop casting method is adopted to achieve a downshifting process by F8BT and DCJTB dyes. 


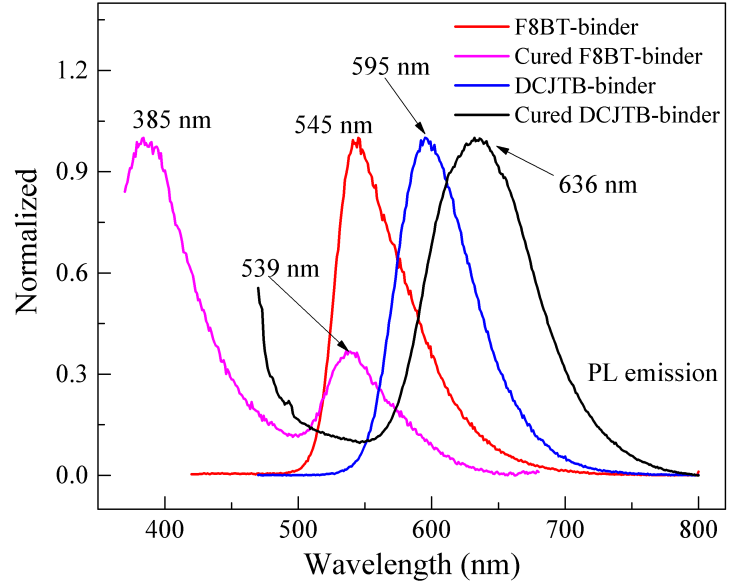

(a)

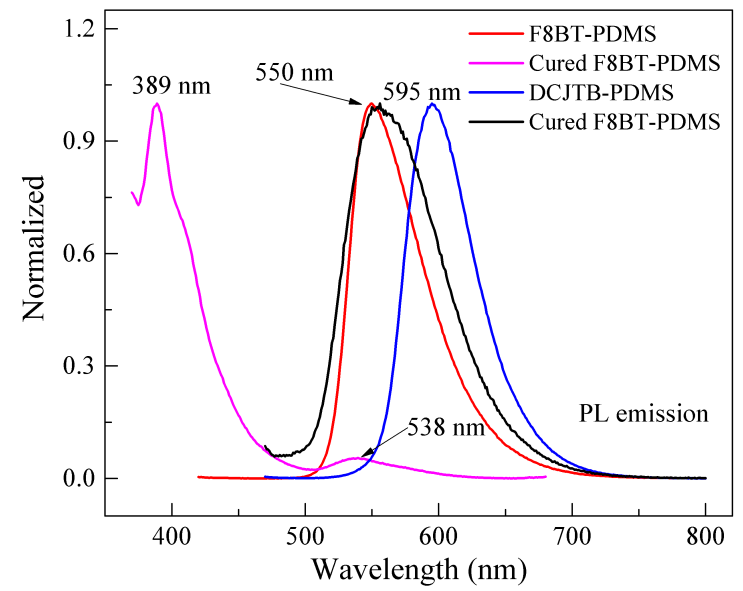

(b)

Figure 3.13: PL emission spectra of F8BT and DCJTB mixed with (a) binder and (b) PDMS before and after curing.

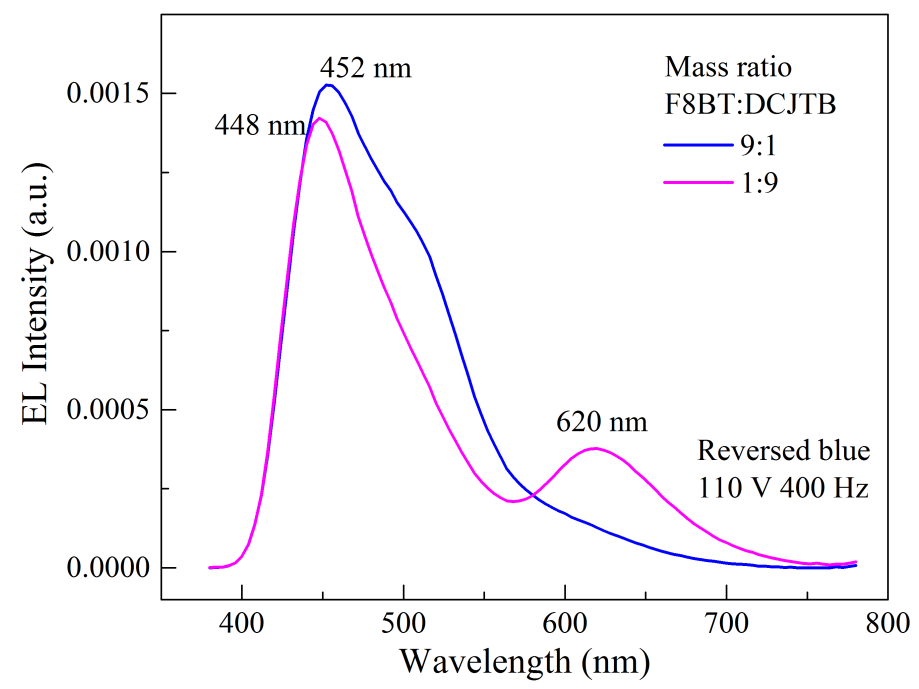

Figure 3.14: EL spectra of reversed blue EL films with spin-coated dye layer with the mass ratio of F8BT:DCJTB at 9:1 and 1:9 respectively.

\subsubsection{Drop casting of a single-dye solution}

A single organic dye dissolved in $\mathrm{CHCl}_{3}$ was drop casted on forward and reversed EL films to verify the downshifting capability of F8BT and DCJTB. In Figure 3.15(a), clear green peaks at about $528 \mathrm{~nm}$ and $536 \mathrm{~nm}$ are observed respectively for the forward and reversed hybrid EL films with a drop of F8BT (forward F8BT, reversed F8BT). Their green emissions can also be seen from the dark field optical images as shown in Figures 3.16(a) 
and 3.16(b) demonstrating the downshifting capability of blue $\mathrm{ZnS}$ emissions by the added F8BT. As illustrated in Figure 3.18(b), some photons of the blue and green emissions from the emitting layer are absorbed by the F8BT layer with an optical band gap of $2.25 \mathrm{eV}$ [155]. After relaxation, singlet and triplet excitons are formed in the F8BT. When the singlet excitons decay radiatively, green light is emitted and gives rise to the EL spectra of forward and reversed F8BT devices in Figure 3.15(a). In addition, the downshifting is more effective for blue photons, as can be inferred from the absorbance spectra in Figure 3.12(a). As a result, the blue peaks of the hybrid devices shift to $450 \mathrm{~nm}$ with the bluegreen lights being absorbed in the F8BT layer. The green peak intensity of forward F8BT is higher than that of reversed F8BT while the blue peak intensities are similar. This shows that the downshifting process is more effective in forward F8BT devices. The drop-casted F8BT in reversed F8BT is visually noticeable from the PET substrate side, which means the F8BT- $\mathrm{CHCl}_{3}$ solution may diffuse into the emitting and dielectric layers upon drying. This leads to less F8BT participating in the downshifting process and thus lowers the intensity of green emissions in reversed hybrid F8BT films.

The forward hybrid EL films with a layer of DCJTB (forward DCJTB) have only one EL emission peak with the peak wavelength of $452 \mathrm{~nm}$ as shown in Figure 3.15(a). Compared with the EL spectra of forward blue, there is a subdued red emission in forward DCJTB. The dark field optical image of forward DCJTB in Figure 3.16(c) shows the existence of circular features with size ranging from hundreds of micrometers to submicrometer. These circular features are further investigated in the SEM cross-sectional images. Holes with various sizes like that in Figure 3.17(a) are identified. A significant number of porosity is seen from Figure 3.17(b). Located at the edge of the coatings, DCJTB in less quantity is observed to have nanorod shapes. Therefore, it is reasonable to believe that the large porosity is caused by the random stacking of DCJTB nanorods. These nanorods may be formed through the minimization of free energy between DCJTB and PET surface where the wettability is low [156]. Meanwhile, the surface holes may be introduced by the 


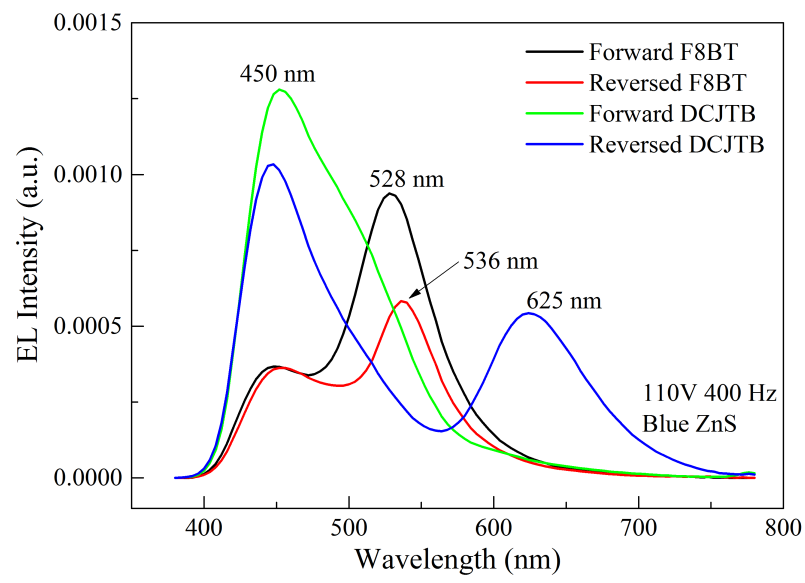

(a)

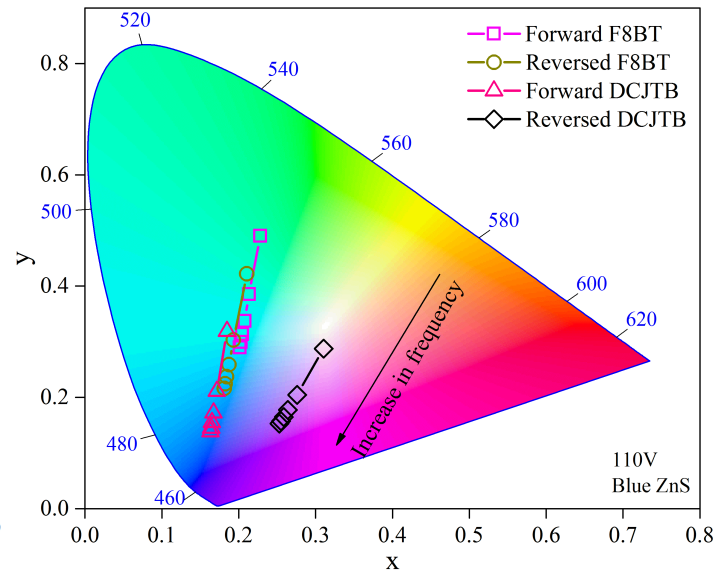

(b)

Figure 3.15: (a) EL spectra at $110 \mathrm{~V}$ and $400 \mathrm{~Hz}$ and (b) CIE at $110 \mathrm{~V}$ and different frequencies for forward and reversed blue EL films with a layer of F8BT or DCJTB organic dye.

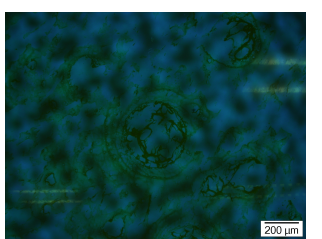

(a)

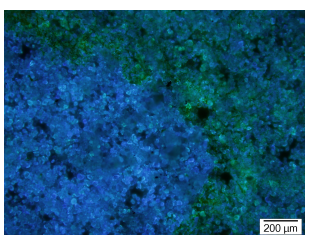

(b)

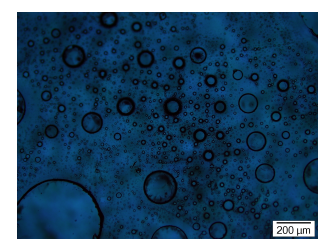

(c)

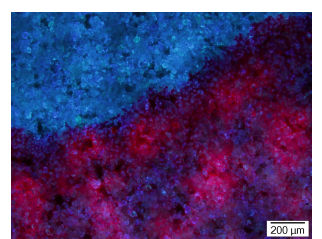

(d)

Figure 3.16: Dark field images of (a) forward F8BT, (b) reversed F8BT, (c) forward DCJTB, and (d) reversed DCJTB operated at $110 \mathrm{~V}$ and $400 \mathrm{~Hz}$.

Marangoni effect [1]. The surface energy at the edges is higher. To minimize the total energy, DCJTB molecules move to the edges and high amount of DCJTB nanorods nucleate and circular patterns are formed. In three dimensional spaces, this process takes places in $\mathrm{x}, \mathrm{y}$ and $\mathrm{z}$ directions and large number of pores is formed. Consequently, when DCJTB is excited, the incident EL lights and converted red emissions are trapped within the pores leading to the insignificant red component in forward DCJTB.

The reversed hybrid films incorporating DCJTB (reversed DCJTB) in Figure 3.15(a), show two EL emission peaks with the peak wavelengths of $445 \mathrm{~nm}$ and $624 \mathrm{~nm}$. The blue shifting of the low wavelength peak indicates the PL absorption of blue-green lights by DCJTB, which is further proven by the red emissions observed from the dark field image 


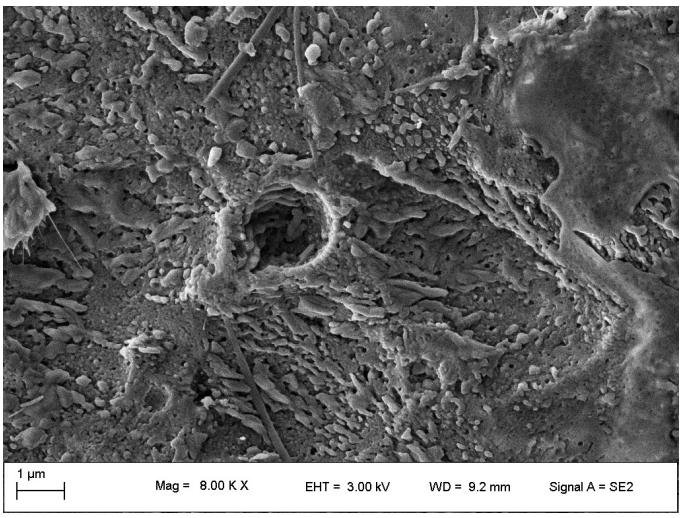

(a)

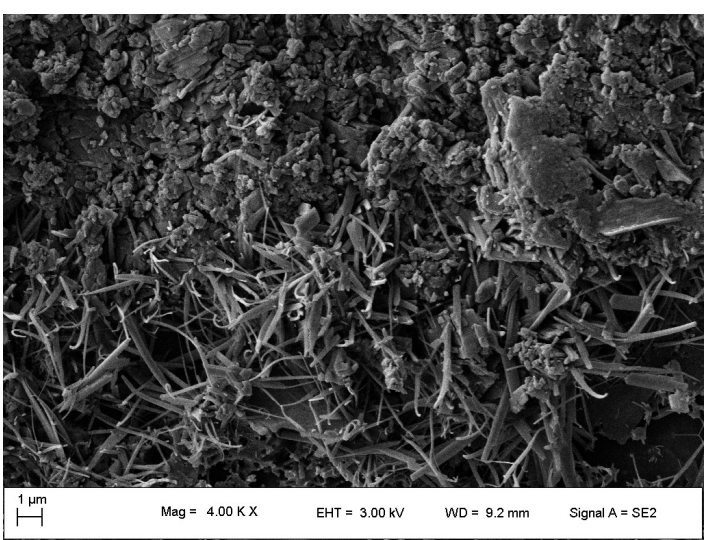

(c)

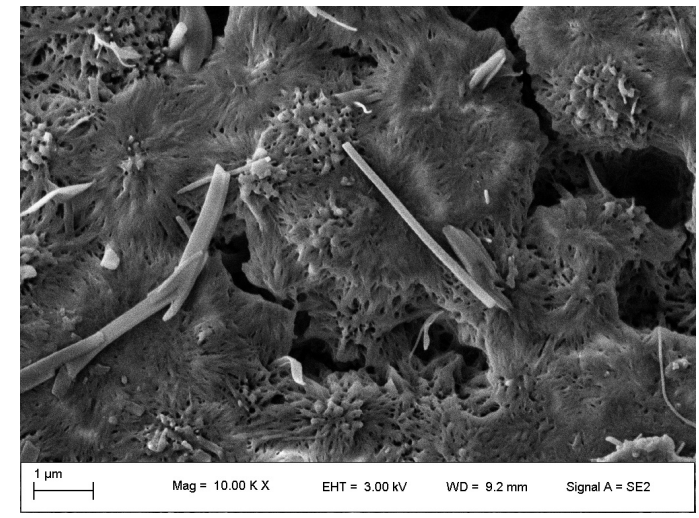

(b)

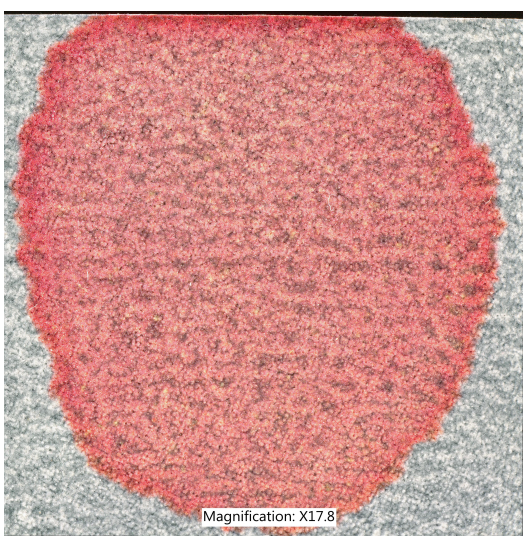

(d)

Figure 3.17: SEM images of (a)-(c) DCJTB over forward EL films; (d) optical images of drop casted DCJTB layer in reversed DCJTB.

in Figure 3.16(d). As illustrated in Figure 3.18(b), DCJTB with the band gap of around $2.01 \mathrm{eV}$ is able to absorb high energy blue and green photons from $\mathrm{ZnS}$ phosphor as well as the converted green emissions from F8BT for the production of red emissions [157]. The dropped casted pattern of forward DCJTB is shown in Figure 3.17(d). The Marangoni effect is clearly seen with deeper red color at the edges. It is also observed that the resulted hybrid film is not in a regular shape, which is a drawback of the drop casting process. The size of this drop casted sample is around $7 \mathrm{~mm}$ at the longest distance. As the spot diameter of the spectraradiometer measurement is $5 \mathrm{~mm}$, it is then difficult to obtain the luminance uniformity, which is a parameter to quantify the uniformity of a large area printed film. 
In Figure 3.15(b), CIE for forward and reversed F8BT follows the same trend with the increase of frequency. At the same operating frequency, the hybrid F8BT devices show a green shift in color due to the downshifting by F8BT. The forward F8BT color becomes green with CIE of $(0.228,0.490)$ when operated at $110 \mathrm{~V}$ and $100 \mathrm{~Hz}$. CIE of forward and reversed DCJTB also varies with frequency from $100 \mathrm{~Hz}$ to $2 \mathrm{kHz}$. The forward DCJTB has only a slight red shift in color output compared to the forward and reversed blue devices while a large red shift is observed for the reversed DCJTB device. This demonstrates that the incorporation of DCJTB into forward EL films fails to yield the desired red component and is therefore not suitable for color tunable EL films. At $100 \mathrm{~Hz}, \mathrm{CIE}$ for the reversed DCJTB is $(0.311,0.288)$ showing near white color. As the operating frequency increases to $2 \mathrm{kHz}$, the downshifting process by DCJTB is enhanced due to the more intense emission from $\mathrm{ZnS}$ and thus a purple color with the CIE of $(0.253,0.153)$ is produced. In view of these results, only reversed EL films are selected for the hybrid devices.

\subsubsection{Drop casting of F8BT and DCJTB mixed solution}

Mass ratio effect

Figure 3.18(a) illustrates the EL spectra of reversed hybrid EL films with a layer of F8BT and DCJTB mixture of different mass ratios operated at $110 \mathrm{~V}$ and $400 \mathrm{~Hz}$. Two clear peaks located at the blue and red regions are observed with the peak wavelengths of $448 \mathrm{~nm}$ and $627 \mathrm{~nm}$ respectively and there is another small hump near the wavelength of $526 \mathrm{~nm}$. As illustrated in Figure 3.18(b), when F8BT and DCJTB are mixed together, F8BT acts as a sensitizer for DCJTB. Short wavelength photons from $\mathrm{ZnS}$ phosphors are first absorbed and downshifted by the sensitizer. This is followed by an energy transfer from F8BT to DCJTB in the form of a further downshifting of F8BT green lights by DCJTB. Consequently, no clear green peaks are observed. As the F8BT:DCJTB mass ratio increases, the $627 \mathrm{~nm}$ peak intensity decreases, showing less red emission by DCJTB. Meanwhile, the intensities of the $448 \mathrm{~nm}$ peak and $526 \mathrm{~nm}$ hump increase with the mass ratio. However, even with the F8BT- 
(a)

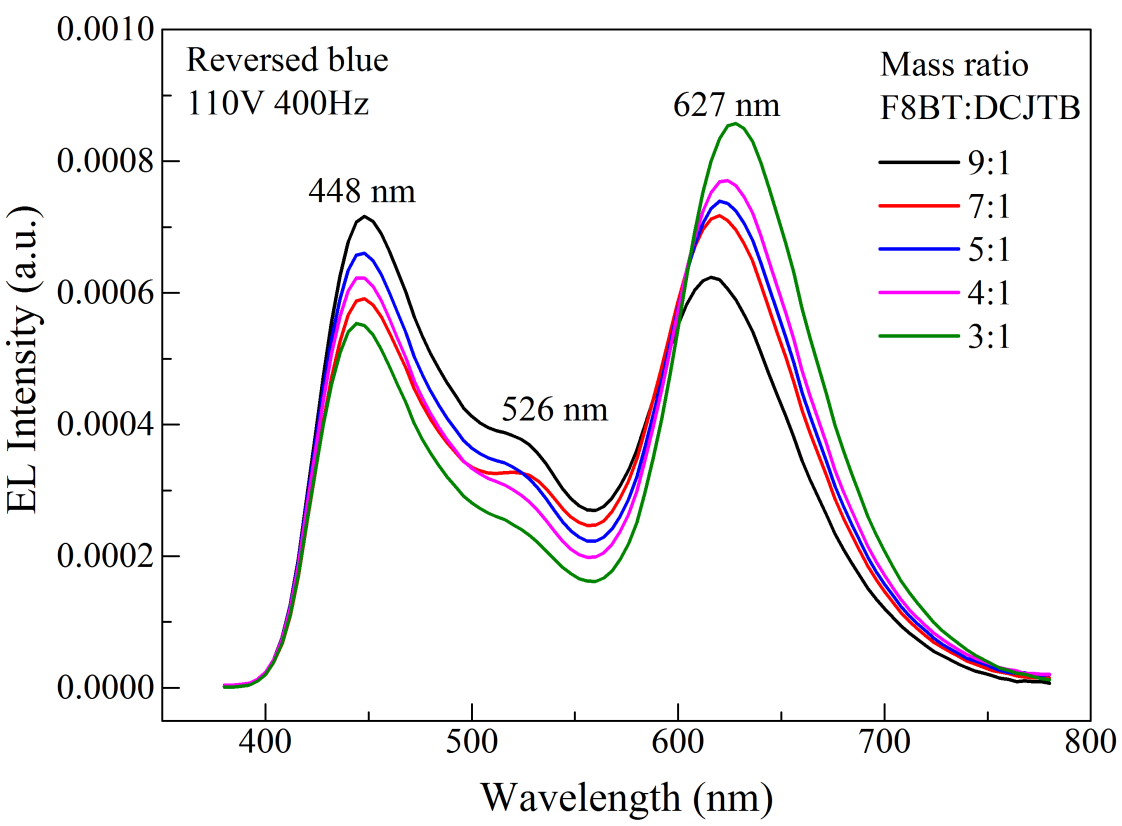

(b)

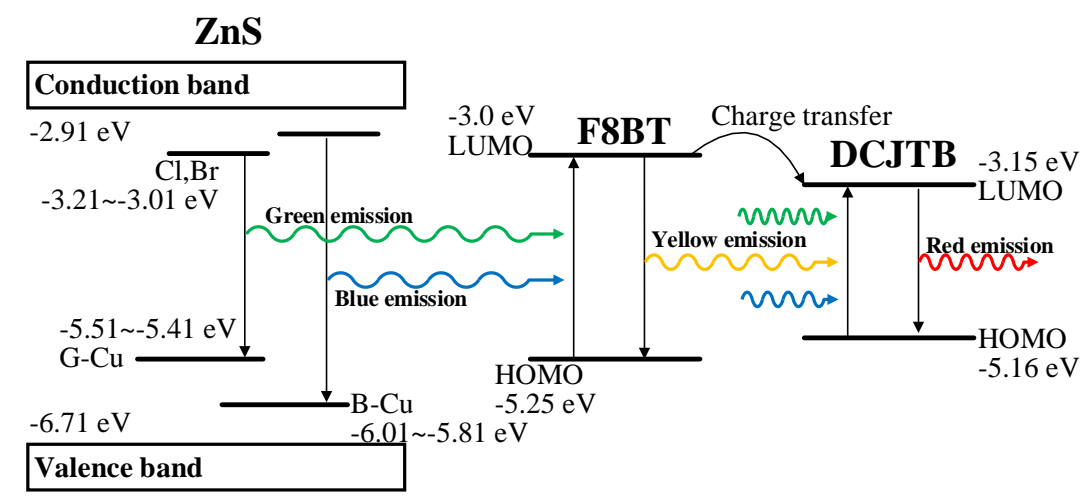

Figure 3.18: (a) EL spectra at $110 \mathrm{~V}$ and $400 \mathrm{~Hz}$ for reversed blue EL films with different mass ratios of F8BT and DCJTB, (b) band diagrams of ZnS, F8BT and DCJTB illustrating the downshifting process.

DCJTB mass ratio of 9:1, the hybrid EL film is still deficit in the $526 \mathrm{~nm}$ emissions. As the final concentration of F8BT and DCJTB mixture vary with the mass ratio as shown in Table 3.1, the dependency of peak intensities is mainly due to the change of the proportion of F8BT and DCJTB in the hybrid devices but may also be affected by the small change of the final concentration.

The increase of mass ratio generally shifts CIE towards blue region except for the mass ratio of 9:1 as shown in Figure 3.19(a). This may be due to the fact that the total dye 


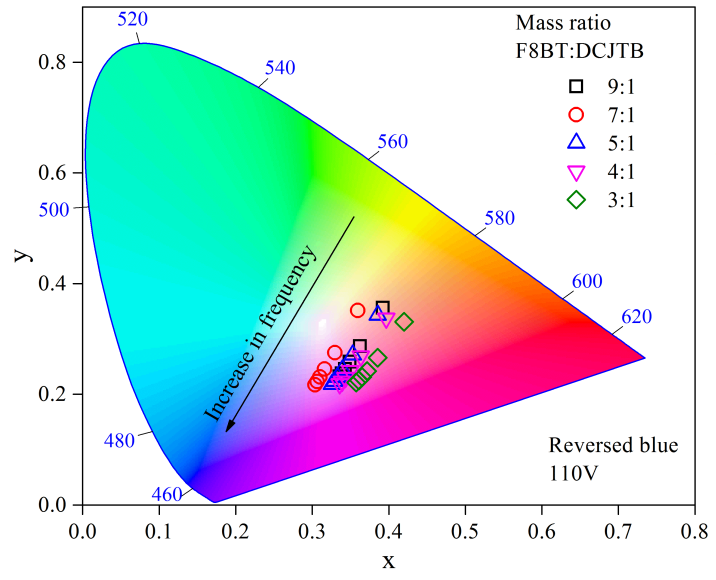

(a)

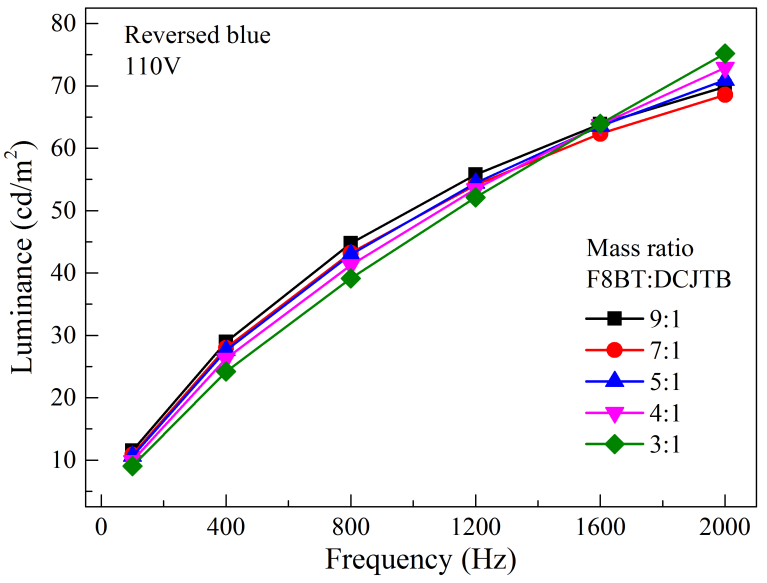

(b)

Figure 3.19: (a) CIE and (b) luminance as a function of frequency at $110 \mathrm{~V}$ for reversed blue EL films with different mass ratios of F8BT and DCJTB.

concentration for the mass ratio of 9:1 is the highest as shown in Table 3.1. A reddish purple color is achieved with the mass ratio of 4:1 when operated at $110 \mathrm{~V}$ and $2000 \mathrm{~Hz}$ and the corresponding CIE is $(0.336,0.219)$. For purplish pink color, the mass ratio needs to be $3: 1$ and the resulted CIE is $(0.386,0.266)$ with the operating voltage at $110 \mathrm{~V}$ and frequency at $400 \mathrm{~Hz}$. The luminance as a function of frequency for hybrid EL films with different F8BT:DCJTB mass ratios are shown in Figure 3.19(b). It can be seen that the increase of mass ratio leads to a slight increase of luminance from $100 \mathrm{~Hz}$ to $1600 \mathrm{~Hz}$. In contrary, at $2000 \mathrm{~Hz}$ increasing F8BT:DCJTB ratio decreases the luminance. As discussed in section 3.3.1, $\mathrm{ZnS}$ phosphors emit an increased portion of blue lights at high frequency and green lights at low frequency. Since DCJTB is more effective in absorbing green light as suggested in Figure 3.12(b), an increase in F8BT:DCJTB ratio will reduce the absorption of green light at low to intermediate frequencies and thus lead to higher luminance. At higher frequencies near $2000 \mathrm{~Hz}$, the emission spectrum of the $\mathrm{ZnS}$ phosphor exhibits a blue shift. The increased blue emission intensity will result in more absorption by F8BT in mixtures with higher F8BT:DCJTB mass ratio. The increased downshifting of blue light thus results in a decrease of the total luminance. 


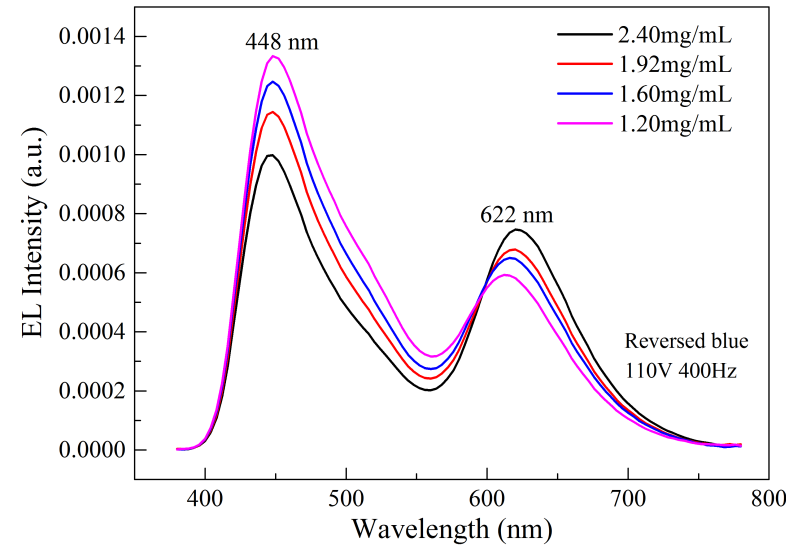

(a)

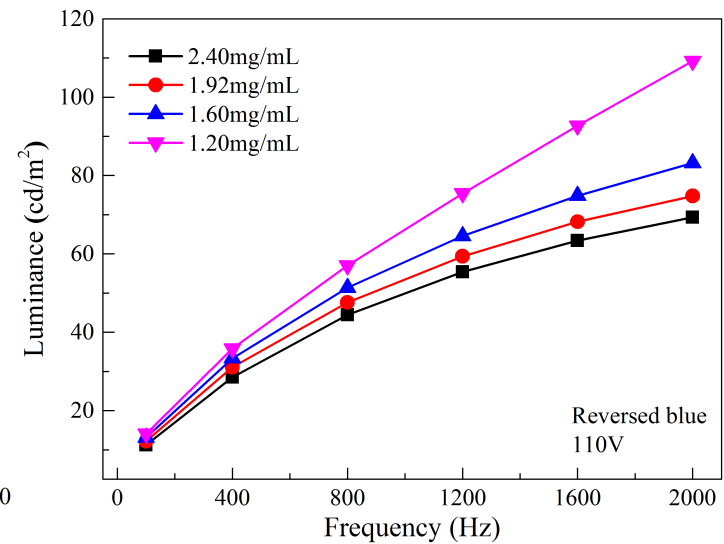

(b)

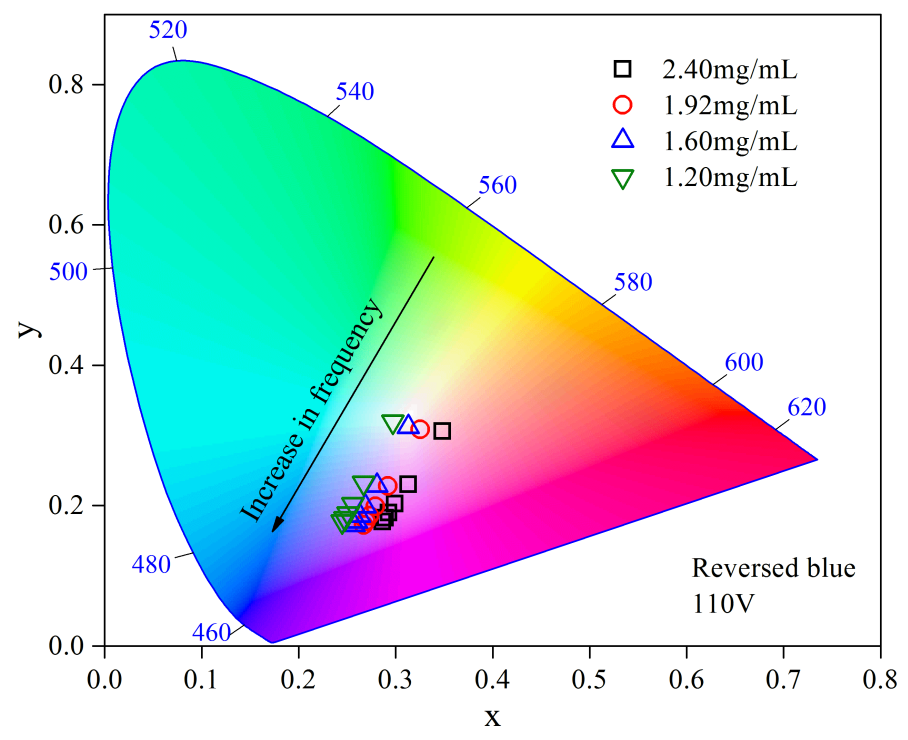

(c)

Figure 3.20: (a) EL spectra at $110 \mathrm{~V}$ and $400 \mathrm{~Hz}$, (b) luminance and (c) CIE as a function of frequency at $110 \mathrm{~V}$ for reversed blue EL films with different concentrations of F8BT and DCJTB mixed solution in $\mathrm{CHCl}_{3}$.

\section{Final concentration effect}

In order to investigate the impact of final mixture concentration on optical properties of the hybrid devices, different amounts of $\mathrm{CHCl}_{3}$ are added into a F8BT and DCJTB mixture with the mass ratio of 3:1. Final concentrations of $2.40,1.92,1.60,1.37$ and $1.20 \mathrm{mg} / \mathrm{mL}$ were achieved and the corresponding EL spectra of the hybrid EL films are shown in Figure 3.20(a). It is observed that decreasing the final concentration increases the blue emission 
intensity but reduces the red peak intensity. No obvious green hump is observed. This is understandable because as the dye mixture becomes more dilute, the solution has less organic dye per unit volume and thus produces less red and green emission. Less downshifting thus causes more blue lights to penetrate the organic layer and the resulting colors are more blueish as the concentration decreases as illustrated in Figure 3.20(c). At $110 \mathrm{~V}$ and 400 $\mathrm{Hz}, \mathrm{CIE}$ of the hybrid EL films with the final concentration of $2.40 \mathrm{mg} / \mathrm{mL}$ is $(0.313,0.230)$ while that for the final concentration of $1.20 \mathrm{mg} / \mathrm{mL}$ is $(0.267,0.232)$. White color with CIE of $(0.313,0.312)$ is achieved for the hybrid devices with the final concentration of $1.60 \mathrm{mg} / \mathrm{mL}$ and operated at $110 \mathrm{~V}$ and $100 \mathrm{~Hz}$ as shown in Figure 3.20(c). However, the luminance at this condition is only $13.13 \mathrm{~cd} / \mathrm{m}^{2}$ and its CCT is $6632 \mathrm{~K}$, which is far from warm white color. The luminance of all these devices increases with frequency as illustrated in Figure 3.20(b). The decrease of the final concentration increases the luminance at all frequencies. Note that there is no crossover of the luminance characteristics as in Figure 3.20(b) because all samples in this plot have the same F8BT:DCJTB mass ratio. It is thus concluded that the red shift for the mass ratio of 9:1 in Figure 3.19(a) is due to the increased concentration of DCJTB causing increased energy transfer from the F8BT sensitizer.

\subsubsection{Aging study}

Aging study of reversed blue EL films

The EL spectra of reversed blue devices under white light aging are shown in Figure 3.22(a). Minimal changes of peak intensity are observed in reversed blue and the luminance in Figure 3.22(b) mostly stays in a steady level with time. This suggests that reversed blue does not suffer from degradation within 120 hr's white light aging. With extended aging time, degradation behaviors may start to emerge. When aged by electrical excitations at $110 \mathrm{~V}$ and $400 \mathrm{~Hz}$, the peak intensity of reversed blue keeps decreasing and the luminance decreases almost linearly with time as shown in Figure 3.22. This degradation is not due to the light aging of EL materials but may be due to the degradation of PEDOT:PSS by 


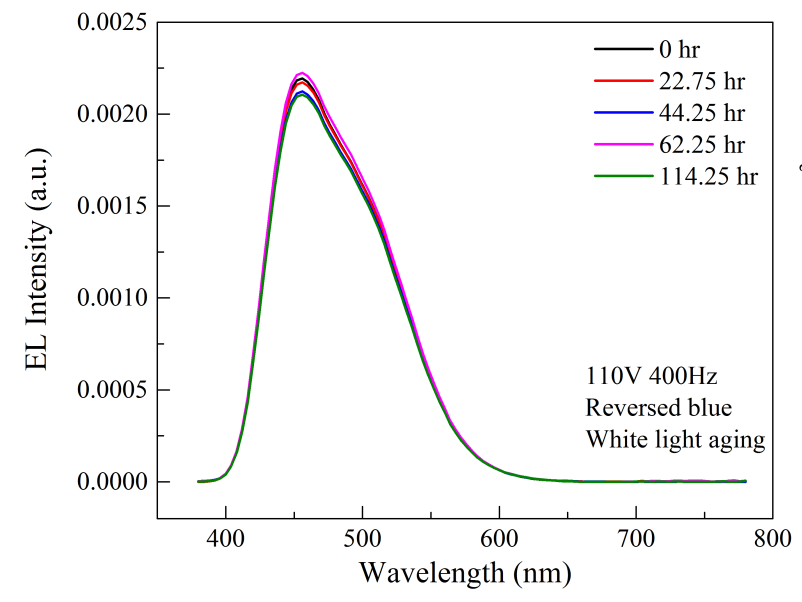

(a)

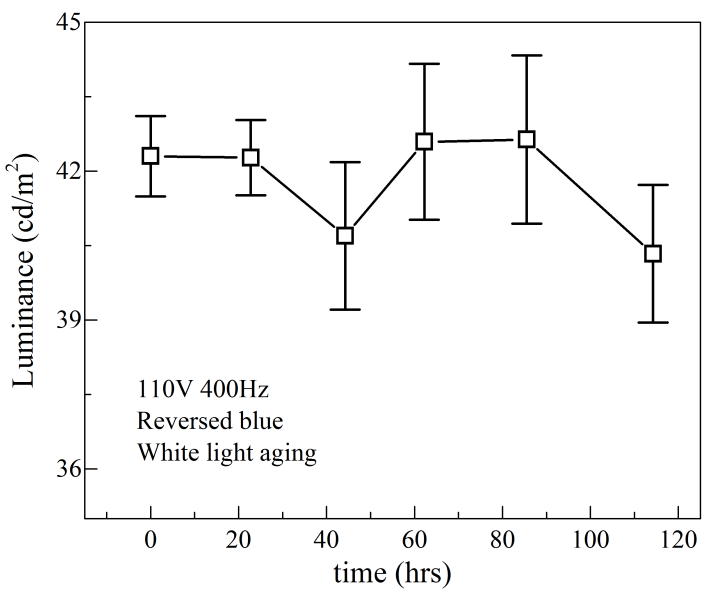

(b)

Figure 3.21: (a) EL spectra and (b) luminance as a function of time for reversed blue EL films exposed to a constant white light source.

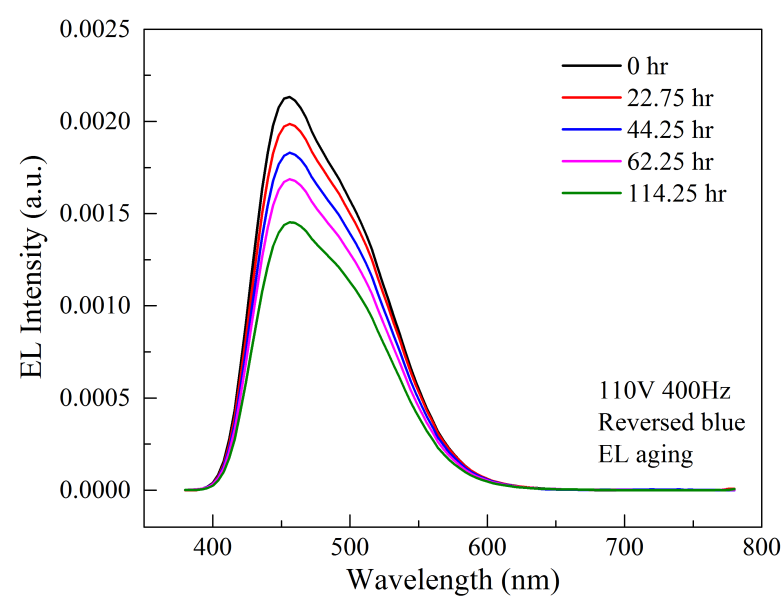

(a)

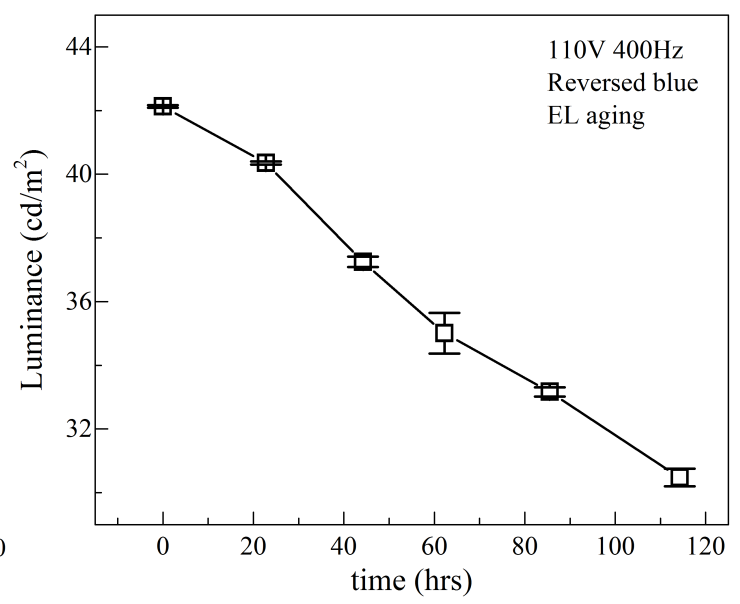

(b)

Figure 3.22: (a) EL spectra and (b) luminance as a function of time for reversed blue EL films operated at $110 \mathrm{~V}$ and $400 \mathrm{~Hz}$.

hot electrons or the degradation of $\mathrm{ZnS}$ phosphors. Further investigations are needed to understand this behavior.

\section{Aging study of hybrid EL films}

During white light aging, the hybrid EL films were removed from the light box from time to time and were turned on briefly at $110 \mathrm{~V}$ and $400 \mathrm{~Hz}$ to measure the EL spectrum and 


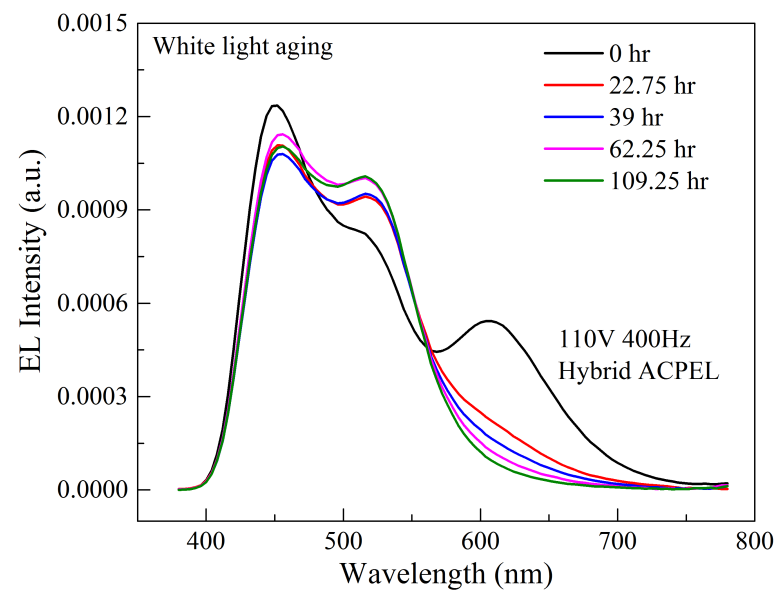

(a)

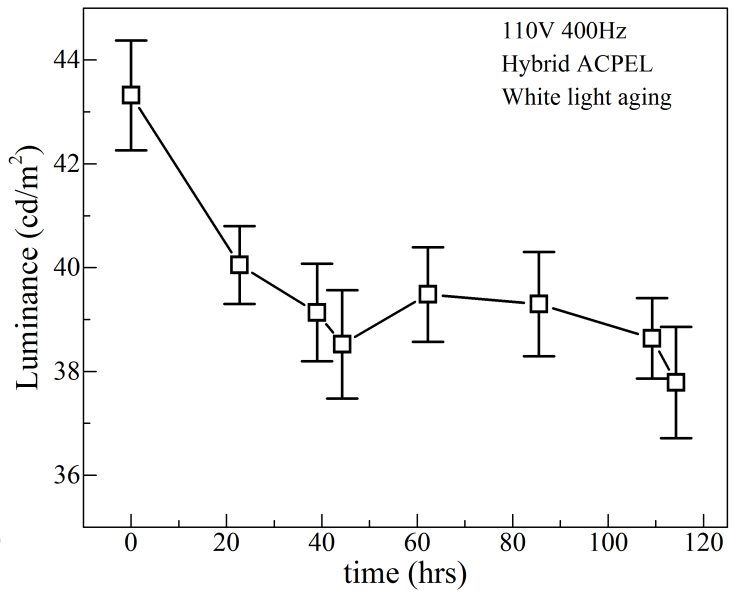

(b)

Figure 3.23: (a) EL spectra and (b) luminance as a function of time for reversed hybrid blue EL films with F8BT and DCJTB layer exposed to a constant white light source.

luminance. In Figure 3.23(a), a red peak contributed by DCJTB and a green shoulder generated by the downshifting in F8BT are initially observed for reversed hybrid EL films with F8BT:DCJTB mass ratio of 9:1. As white light aging progresses, the red peak intensity decreases continuously until it diminishes. This suggests that DCJTB degrades readily under white light illumination. However, the green shoulder becomes a green peak with aging time and the intensity of this peak increases thereafter. This is because with less DCJTB, energy transfer from F8BT is reduced leading to higher green emission. This change in color also indicates that F8BT is more stable under white light aging. In Figure 3.23(b), as DCJTB degrades, the device luminance decreases by about $5 \mathrm{~cd} / \mathrm{m}^{2}$ and becomes stable after 40 hours.

The change of optical properties of the hybrid EL films under EL aging is different from that under white light aging. As shown in Figure 3.24(a), the red peak intensity reduces with time continuously under EL aging. With less DCJTB and less sensitizing effect, the green peak intensity increases during the first 22.75 hours but decreases thereafter. This change of the green peak intensity can be due to two reasons. First, the F8BT also degrades under EL aging but the degradation rate is much less than that of DCJTB. On the other 


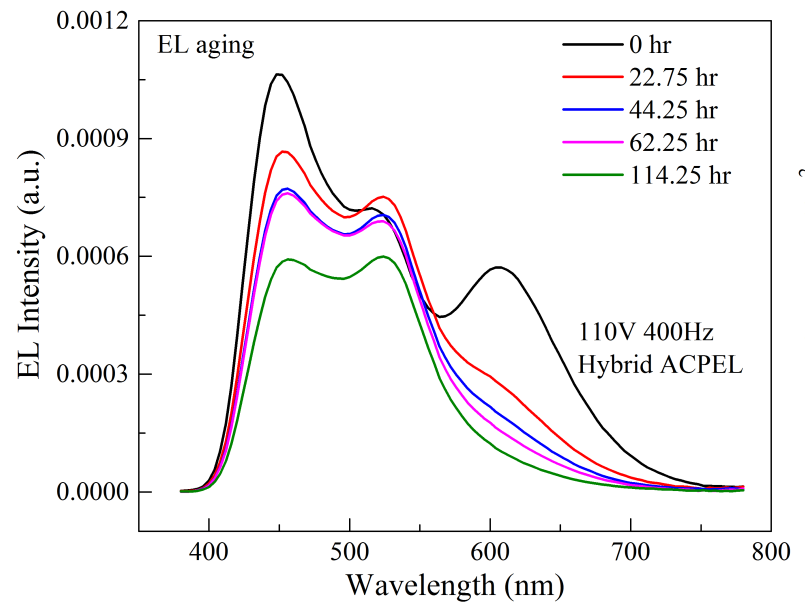

(a)

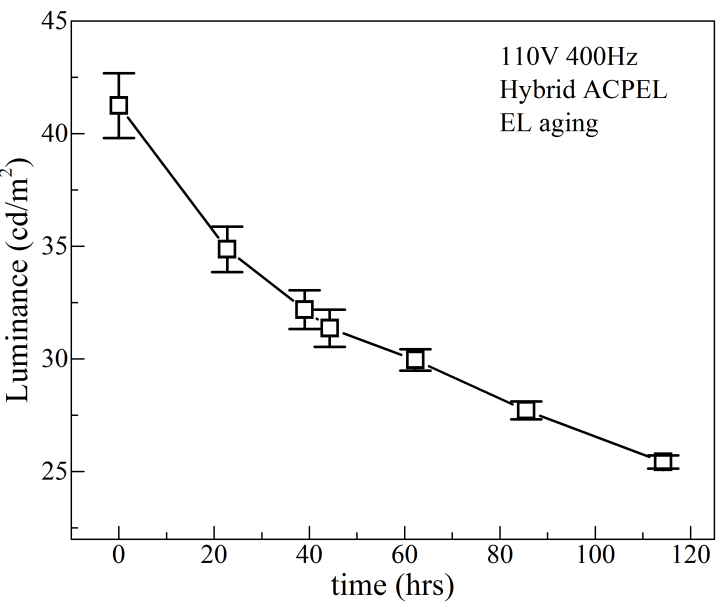

(b)

Figure 3.24: (a) EL spectra and (b) luminance as a function of time for reversed hybrid blue EL films with F8BT and DCJTB layer operated at $110 \mathrm{~V}$ and $400 \mathrm{~Hz}$.

hand, since little degradation was observed for F8BT under white light aging, reduced incident blue EL may have resulted in decreased green emission by F8BT under EL aging. Further studies, however, will have to be conducted to understand the EL aging mechanism. Figure 3.24(b) shows that unlike white light aging, there is a monotonic decrease of device luminance. After around 114 hours of EL aging, the luminance decreased by $39 \%$ of the initial value.

\subsection{Conclusion}

In this study, F8BT and DCJTB organic dyes have been investigated by PL and EL methods for the downshifting capabilities of blue emission from $\mathrm{ZnS}$ phosphors. EL excited green and red emissions in F8BT and DCJTB are obtained respectively through downshifting and sensitizer-downshifting mechanisms. Forward EL films are unsuitable for color tuning due to low red light output from the drop-casted layer. For the reversed device structure, emissions from the hybrid EL films can be tuned through the adjustment of F8BT:DCJTB mass ratio, total dye solution concentration and the excitation frequency. Increasing the mass ratio enhances the blue and green emission peaks but the red peak intensity is reduced. 
Similarly, diluting the F8BT and DCJTB mixture suppresses the green and red emission peaks and enhances the blue peak. Colors ranging from green to white can be obtained from the reversed hybrid EL films. EL and white light aging results show that DCJTB degrades under both conditions while F8BT does not degrade under white light aging. However, the luminance of white light is only $13.13 \mathrm{~cd} / \mathrm{m}^{2}$. An improvement of printing process is also needed to enable a large area processing. 


\section{Chapter 4}

\section{PRINTED HYBRID EL FILMS BY WET STAMPING AND BAR COATING PROCESSES}

\subsection{Introduction}

In Chapter 3, a simple and cost-effective drop casting method was developed to coat a layer of F8BT and DCJTB mixture on forward and reversed EL films. A white color with CIE of $(0.313,0.312)$ was achieved when the hybrid devices were operated at $110 \mathrm{~V}$ and $100 \mathrm{~Hz}$. However, the luminance in this operating condition was only $13.13 \mathrm{~cd} / \mathrm{m}^{2}$. The other drawback of the drop casting process is that the shape and size of the coated layer are not controllable. In order to obtain a uniform white/warm-white hybrid EL films with controllable shapes, modified wet stamping and bar coating techniques are adopted in this chapter and F8BT is replaced by fluorescein to perform coating process with dye in ethanol solutions. The process conditions are tuned to produce coatings with the highest uniformity. The mass ratios and concentrations of the dye solution are altered to obtain a white and warm white emissions.

\subsection{Experimental details}

\subsubsection{Modified wet stamping process}

As concluded in Chapter 3, reversed EL films are more suitable to fabricate hybrid devices through the downshifting of organic dye layers [158]. The detailed screen printing process, device structure and materials used in reversed EL films can be referred to in Section 3.2. A modified wet stamping process for the deposition of a dye layer with controlled shape and size is constructed as shown in Figure 4.1. Two small molecules were used as the down- 
shifting materials: fluorescein (Lumtec LT-F0089) and DCJTB. The molecular structure of fluorescein is shown in Figure 4.2(a). These two organic dyes were dissolved in ethanol (95.0\%, Sigma-Aldrich). A commercial rubber stamp with the size of $20 \mathrm{~mm} \times 20 \mathrm{~mm}$ was ultrasonically cleaned in ethanol for $10 \mathrm{~s}$ using water bath FB15055 from Fisher Scientific, followed by $365 \mathrm{~nm}$ UV exposure for 5 min under Blak-Ray B-100AP high intensity UV lamp. The surface-treated stamp was then attached to a stainless steel block with a prede-

fined mass $w$ for the applying of certain pressure. To dip coat a layer of dye molecules, the stamp was immersed in the dye-ethanol solution for $3 \mathrm{~s}$ and held in air with a tilt angle of $45^{\circ}$ to dry off the coating for a predefined time $\left(t_{d}\right)$. Thereafter,the coated stamp was placed in contact with the transparent electrode of reversed EL films which were pre-heated to a predefined temperature $(T)$ controlled by a AREC hotplate from VELP SCIENTIFICA. After a predefined stamping time $\left(t_{s}\right)$, the stamp was removed from the sample and ethanol was evaporated at temperature $T$ to form an organic dye layer.

\subsubsection{Optimization of wet stamping conditions using fluorescein}

To obtain the optimal wet stamping conditions, $2 \mathrm{mg} / \mathrm{mL}$ fluorescein solution was prepared by dissolving $32.7 \mathrm{mg}$ of fluorescein in $16.35 \mathrm{~mL}$ of ethanol to be stamped over reversed blue EL films. The experimental matrix was designed according to Table 4.2.2. For the first experiment, wet stamping of fluorescein was performed using different mass values $(0.20$, $0.40,0.72,0.99,1.62 \mathrm{~kg})$ with the other conditions kept constant $\left(t_{s}=15 \mathrm{~s}, T=60^{\circ} \mathrm{C}, t_{d}=10\right.$ s). Secondly, to obtain the best stamping time, constant $w(0.99 \mathrm{~kg}), T\left(60{ }^{\circ} \mathrm{C}\right)$ and $t_{d}(10 \mathrm{~s})$ were used with varied $t_{s}(5,10,15,20,25,40 \mathrm{~s})$. After that, different hot plate temperatures $\left(30,40,50,60,70,80,90^{\circ} \mathrm{C}\right)$ were tested to obtain the optimal temperature for the wet stamping process. The other conditions were $w=0.99 \mathrm{~kg}, t_{s}=15 \mathrm{~s}, t_{d}=10 \mathrm{~s}$. Lastly, different $t_{d}(5,10,15,20,25,30,40 \mathrm{~s})$ was tested with fixed $w(0.99 \mathrm{~kg}), T\left(60{ }^{\circ} \mathrm{C}\right)$ and $t_{s}(15 \mathrm{~s})$. 


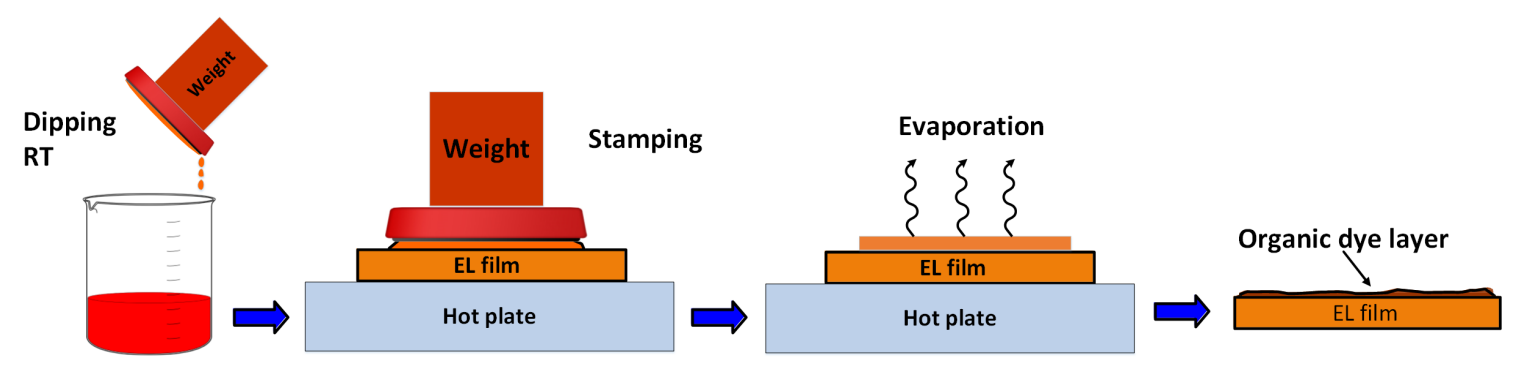

Figure 4.1: Modified wet stamping process integrated with dip coating and temperature control. RT: room temperature.

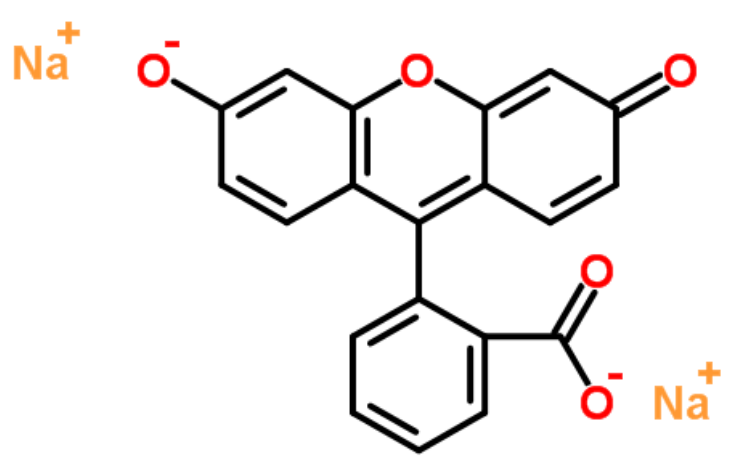

(a)

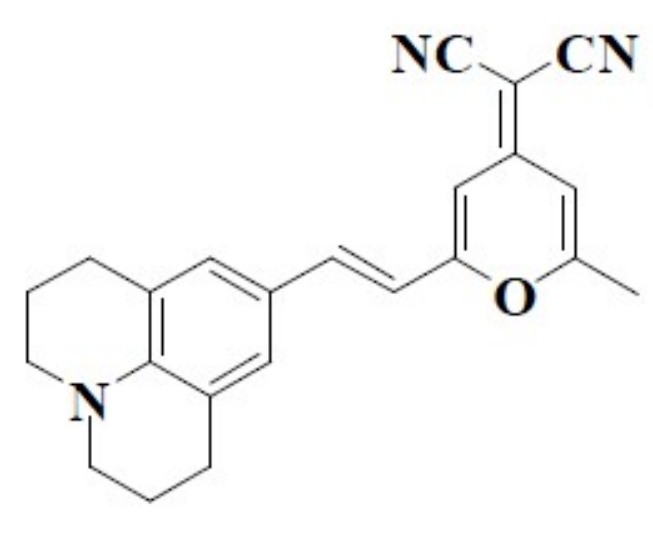

(b)

Figure 4.2: Molecular structures of (a) fluorescein sodium and (b) DCM2.

Table 4.1: Experimental matrix for the optimization of various experiment conditions.

\begin{tabular}{cc}
\hline Parameters & \multicolumn{1}{c}{ Values } \\
\hline $\mathrm{w}(\mathrm{kg})$ & $0.20,0.40,0.72,0.99,1.62$ \\
$\mathrm{t}_{\mathrm{s}}(\mathrm{s})$ & $5,10,15,20,25,40$ \\
$\mathrm{~T}\left({ }^{\circ} \mathrm{C}\right)$ & $30,40,50,60,70,80,90$ \\
$\mathrm{t}_{\mathrm{d}}(\mathrm{s})$ & $5,10,15,20,25,30,40$ \\
\hline
\end{tabular}


Table 4.2: Various mass ratios and concentrations of mixed DCJTB and fluorescein solution.

\begin{tabular}{lll}
\hline Experiment & \multicolumn{1}{c}{ Mass ratio } & Concentration $(\mathrm{mg} / \mathrm{mL})$ \\
\hline 1 & $7: 1,5: 1,3: 1,1: 1,1: 3,1: 5,1: 7$ & 2 \\
2 & $1: 1$ & $2,3,4,5,6$ \\
\hline
\end{tabular}

\subsubsection{Fabrication of white EL films with DCJTB and fluorescein mixture by wet stamping process}

With the optimal experimental conditions of $w=0.99 \mathrm{~kg}, T=60{ }^{\circ} \mathrm{C}, t_{s}=10 \mathrm{~s}$ and $t_{d}=15 \mathrm{~s}$ determined from the previous experiment in section 4.2.2, the mass ratio and concentration of mixed DCJTB and fluorescein solution were varied to fabricate white hybrid EL films by downshifting mechanism from blue $\mathrm{ZnS}$ phosphors. The mixed solution was achieved by dissolving predefined amount of DCJTB and fluorescein powders in ethanol. As shown in Table 4.2, the first experiment kept the dye concentration to be constant at $2 \mathrm{mg} / \mathrm{mL}$ while the mass ratios of these two dyes were varied to be $7: 1,5: 1,3: 1,1: 1,1: 3,1: 5$ and 1:7. In the second experiment, the dye solution with the mass ratio of 1:1 was taken from the first experiment. By adding suitable amount of ethanol and dyes, different dye concentrations $(2,3,4,5$ and $6 \mathrm{mg} / \mathrm{mL})$ were obtained. For the first experiment, the optimal conditions were used. In the second experiment, $w$ was adjusted to $0.4 \mathrm{~kg}$ as it produced thicker dye layer and thus stronger downshifted emissions.

\subsubsection{Fabrication of warm white hybrid films by wet stamping process}

As observed in Section 4.2.3, DCJTB saturates in ethanol at concentrations above $5 \mathrm{mg} / \mathrm{mL}$. Therefore, $4 \mathrm{mg} / \mathrm{mL}$ of DCJTB was prepared to fabricate the multilayered hybrid EL films. One to seven layers of DCJTB coatings were stamped over green EL films with $w=0.4 \mathrm{~kg}$, $T=60{ }^{\circ} \mathrm{C}, t_{s}=10 \mathrm{~s}$ and $t_{d}=10 \mathrm{~s}$. The alignment for each printing was achieved manually by 
visual adjustment. Additionally, $4 \mathrm{mg} / \mathrm{mL}$ of DCJTB-fluorescein solution with the mass ratio of 1:1 was prepared for wet stamping. One to five layers of such mixed solution was coated onto reversed green EL films using the same conditions.

\subsubsection{Fabrication of large-area warm white hybrid films by bar coating technique}

The coating process of bar coating techniques is similar to the blade coating process which is introduced in Section 2.2.5. A distinguished feature is the wire-wrapped bar. The deposition finish is controlled partly by the wire diameter or thread thickness. As the wet stamping has a limited coating area and selective materials and solvents, the successful demonstration of bar coating of hybrid EL films will have a great value for the large-scale and low cost printing process and for the commercialization of this type of hybrid devices. Here we used DCM2 dye (Lumtec LT-E702) as the red emission downshifting material and fluorescein as the green emission downshifting material. The structure of DCM2 is shown in Figure 4.2(b). To formulate the dye solution, DCM2 was first dissolve in $\mathrm{CHCl}_{3}$, followed by adding ethanol to create a mixed solution. Thereafter, fluorescein was added into the mixed solution to form the printing agent with a designed mass ratio of DCM2 and fluorescein. To first obtain the best processing conditions, $4 \mathrm{mg} / \mathrm{mL}$ of DCM2-fluorescein solution with the mass ratio of 3:1 was formulated. Bars with the thread thicknesses of $40,60,80,100$ and $120 \mu m$ were used for the bar coating process of the above solution on reversed green EL films with the printing speed of $100 \mathrm{~mm} / \mathrm{s}$. Another experiment was conducted using the $120 \mu \mathrm{m}$ bar with the various printing speeds of 10,50 and $100 \mathrm{~mm} / \mathrm{s}$. To study the concentration effects on the printing quality and obtain a warm white color, a layer of 1.0, 2.0 and $4.0 \mathrm{mg} / \mathrm{mL}$ solution with only DCM2 was printed using $80 \mu \mathrm{m}$ bar and $50 \mathrm{~mm} / \mathrm{s}$. 2, 4 and 5 layers of $4.0 \mathrm{mg} / \mathrm{mL}$ DCM2 solution were also bar coated to study the thickness effects. Eventually $8.0 \mathrm{mg} / \mathrm{mL}$ DCM2-fluorescein solution with the mass ratio of 5:1, $15 \mathrm{mg} / \mathrm{mL}$ DCM2-fluorescein solution with the mass ratio of $9: 1$ and $15 \mathrm{mg} / \mathrm{mL}$ DCJTB-fluorescein solution with the mass ratio of 9:1 were formulated and printed indi- 
vidually over reversed green EL films using the $80 \mu \mathrm{m}$ bar and with the printing speed of $50 \mathrm{~mm} / \mathrm{s}$ to obtain a warm white large area hybrid lighting source.

\subsubsection{Device measurement and characterization}

Photoluminescence (PL) emission spectra of the organic dyes in ethanol solution and on PET films were acquired using a Fluorolog-3 PL system. The absorbance spectra were obtained by a Shimadzu UV-2450 UV-VIS spectrometer. EL spectra, luminance and CIE were measured using the SpectraWin software of PR655 spectroradiometer from Photo Research. The measurement was performed at a $2^{\circ}$ observer angle with a spot size of about $5 \mathrm{~mm}$ in a dark enclosure and at ambient conditions. The power luminous efficacy was obtained by dividing the luminous flux by the electrical power. The luminous flux was measured using a halfmoon integrating sphere from Labsphere and the electrical power was measured by a YOKOGAWA WT210 digital power meter. The AC sinusoidal excitation voltages for the printed EL film were supplied by a Pacific 105-AMX power source without any DC bias. To calculate the emission uniformity of the hybrid devices, the luminance/CCT of five positions was measured with four at the corners and one in the center. The uniformity follows:

$$
\text { Uniformity }=(1-\sigma / \mu) * 100 \%
$$

where $\sigma$ is the standard deviation and $\mu$ is the mean luminance/CCT of the measured five positions. EL and white light aging were performed to study the durability of fluorescein and DCJTB in white hybrid EL films by wet stamping process. In order to reduce experimental errors, optical measurements were taken on five positions of the stamped film, one in the center and four at the corners. EL aging was carried out by operating the devices continuously at $110 \mathrm{~V}$ and $400 \mathrm{~Hz}$ in a dark room at $25{ }^{\circ} \mathrm{C}$ and $70 \%$ relative humidity. For

white light aging, a fluorescent white light box with the radiance power of $4.3 \mathrm{Wsr}^{-1} \mathrm{~m}^{-2}$ 


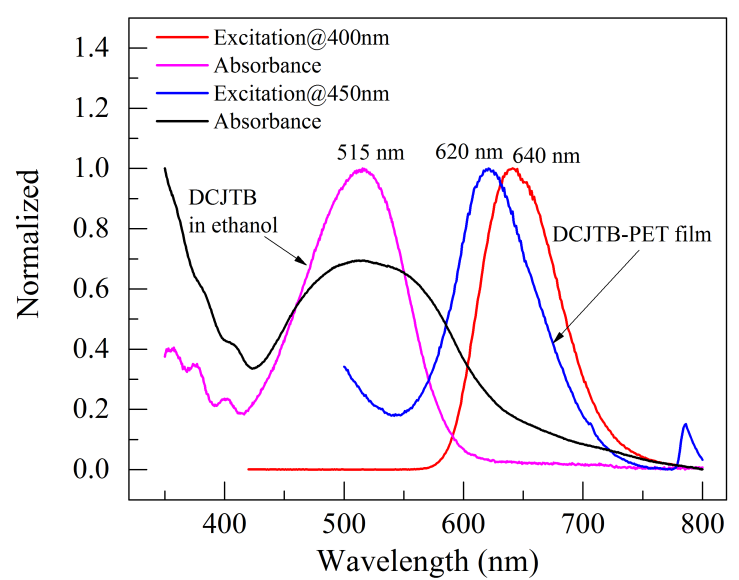

(a)

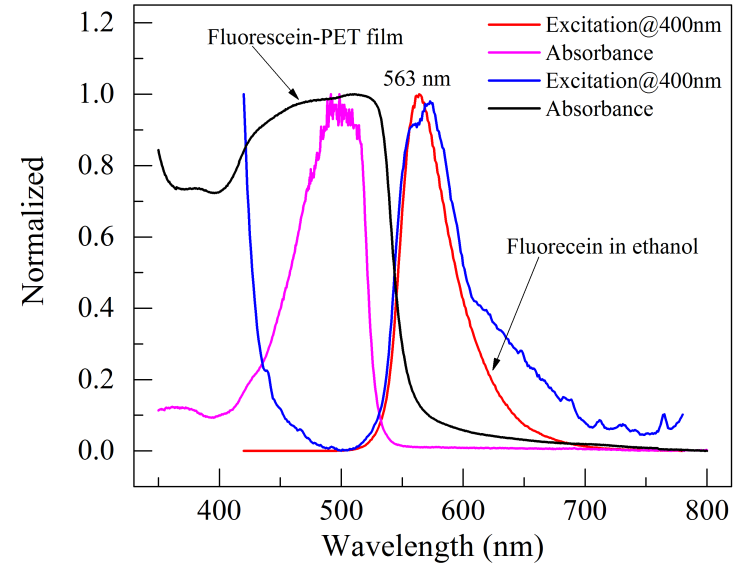

(b)

Figure 4.3: Normalized PL emission and absorbance spectra of (a) DCJTB-ethanol solution and DCJTB-PET film and (b) fluorescein-ethanol solution and fluorescein-PET film.

was used to age the hybrid EL films at $25{ }^{\circ} \mathrm{C}$ and $70 \%$ relative humidity in a dark room.

\subsection{Results and discussion}

\subsubsection{Optimization of wet stamping conditions}

The PL emission and absorbance spectra of DCJTB and fluorescein in ethanol solution and on PET films are shown in Figure 4.3. When photo-excited, DCJTB emits red lights with the peak wavelengths of $620 \mathrm{~nm}$ in ethanol solution and $640 \mathrm{~nm}$ on PET film. The absorbance spectrum of the DCJTB-ethanol solution has a peak wavelength of $515 \mathrm{~nm}$ while the DCJTB-PET film exhibits a broader absorption capability with a similar peak position. In Figure 4.3(b), photo-excited fluorescein has emission spectra with the peak wavelength of around $563 \mathrm{~nm}$ both in ethanol solution and on PET film. The fluoresceinethanol solution is able to absorb lights with the peak wavelength of around $500 \mathrm{~nm}$ while the fluorescein-PET film can be excited by a broader range of visible lights ranging from $400 \mathrm{~nm}$ to $530 \mathrm{~nm}$ with comparable absorbances. Therefore, both DCJTB and fluorescein can be photo-excited by the blue emission from $\mathrm{ZnS}$ phosphors, whose EL spectrum is presented in Chapter 3. Moreover, fluorescein is capable of sensitizing DCJTB effectively. 
The effects of $T, w, t_{s}$ and $t_{d}$ on the luminance uniformities of wet stamped hybrid EL films are shown in Figure 4.4. The luminance uniformity increases with temperature till $60{ }^{\circ} \mathrm{C}$ and decreases thereafter as illustrated in Figure 4.4(a). The highest uniformity is obtained at $60{ }^{\circ} \mathrm{C}$ with the value of $97.25 \%$. Therefore, $60{ }^{\circ} \mathrm{C}$ is the best temperature for the wet stamping process at which the evaporation and coating rate are optimal. At temperatures higher than $60{ }^{\circ} \mathrm{C}$, the evaporation rate is so high that fluorescein does not have enough time to spread over the stamping area, which gives forth lower uniformity. At temperatures lower than $60{ }^{\circ} \mathrm{C}$, the amount of solution coated on the stamp is high causing stains remaining within the contact region when stamps are removed. The effect of weight exhibits a similar trend. When pressure is applied by the $0.99 \mathrm{~kg}$ weight, the hybrid device has the highest luminance uniformity of $94.28 \%$. When the mass is less than $0.99 \mathrm{~kg}$, the increase of weight introduces higher pressure over the dye solution and pushes it towards the stamp edge, which increases the luminance uniformity. However, when the weight keeps increasing and exceeds $0.99 \mathrm{~kg}$, the pressure is so high that most of the dye solution is pushed to the stamp edge leaving less coatings in the center of the stamp. That is why it is observed that the luminance in the center is higher with less emission losses for the samples stamped with $16.2 \mathrm{~N}$ weight. In Figure 4.4(b), the relationship between the luminance uniformity and $t_{s}$ or $t_{d}$ is not straightforward. However, it is observed that $t_{s}=10$ $\mathrm{s}$ and $t_{d}=15 \mathrm{~s}$ are the two optimal conditions for the wet stamping process which lead to the highest uniformity values of $96.24 \%$ and $94.14 \%$ respectively.

\subsubsection{Wet stamped hybrid films with DCJTB and fluorescein}

\section{Effects of mass ratio}

In order to achieve white emission from the hybrid EL films, it is necessary to coat both DCJTB and fluorescein to produce red and green components. Our approach is to mix both dyes in ethanol and wet stamp it in just one layer. As such, the DCJTB-fluorescein mass ratio plays a critical role. From the EL spectra of the hybrid devices with different mass ra- 


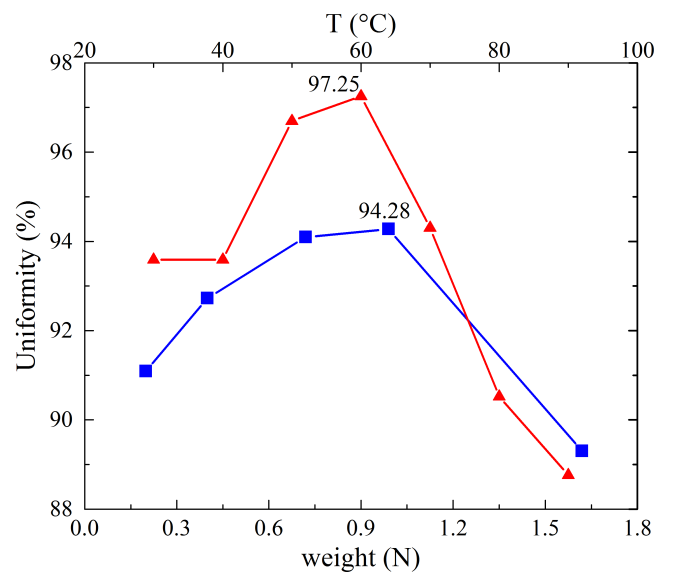

(a)

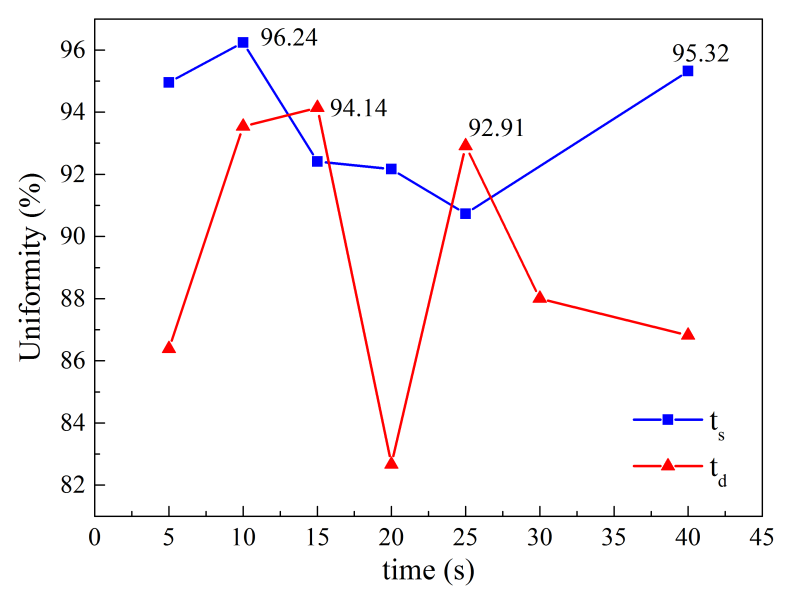

(b)

Figure 4.4: Uniformity of reversed EL films deposited with a layer of fluorescein using wet stamping process with different experimental conditions.

tios in Figure 4.5, there is a blue peak at around $450 \mathrm{~nm}$ from blue $\mathrm{ZnS}$ phosphor emission, another green peak at around $532 \mathrm{~nm}$ downshifted from fluorescein and a red shoulder at around $610 \mathrm{~nm}$ from DCJTB. With the increase of fluorescein proportion, the blue peak sees a slight blue shift while the relative intensities of both the green peak and red shoulder increase. As shown in Figure 4.3(b), fluorescein is more effective in absorbing blue-green lights and thus with the increased amount of fluorescein the blue peak experiences a blue shift. On the other hand, the red DCJTB is more effective in absorbing green-yellow lights as indicated in Figure 4.3(a). With the increase of the fluorescein ratio, the amount of green-yellow lights emitted by fluorescein is increased and thus the red emission is inten- 


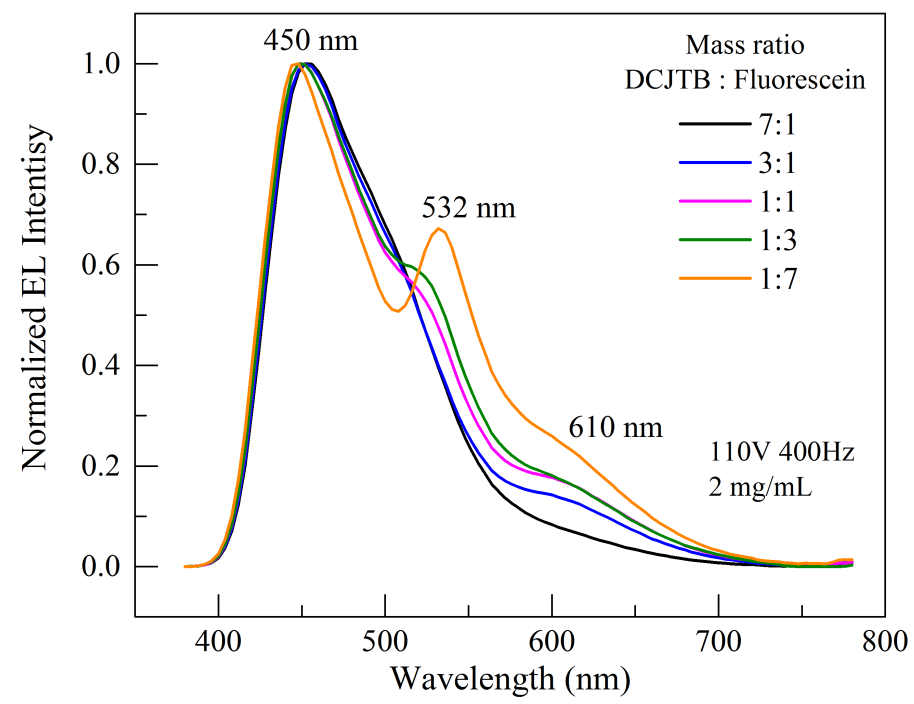

Figure 4.5: EL spectra at $110 \mathrm{~V}$ and $400 \mathrm{~Hz}$ for hybrid EL films with fixed dye concentration at $2 \mathrm{mg} / \mathrm{mL}$ and different DCJTB:fluorescein mass ratios.

sified. However, the overall green and red emissions are still weak which is probably due to the small thickness of the dye layer or the low concentration of the dye-ethanol solution.

The emission mechanism of the hybrid EL films incorporating downshifting organic dyes is illustrated in Figure 4.6. When excited, doped $\mathrm{Cu}$ forms two trapped states: one contributing to green emission at the energy level of -5.51 to $-5.41 \mathrm{eV}(\mathrm{G}-\mathrm{Cu})$ and the other contributing to blue emission at the energy level of -6.01 to $-5.81 \mathrm{eV}(\mathrm{B}-\mathrm{Cu})$ [92]. Free charge carriers are generated by field emission of $\mathrm{Cu}_{\mathrm{x}} \mathrm{S}$ needles as proposed by Fischer [46]. Electrons trapped in the donor sites combine with holes in $\mathrm{G}-\mathrm{Cu}$ and $\mathrm{B}-\mathrm{Cu}$ sites to emit green and blue lights. These emissions from $\mathrm{ZnS}$ phosphors excite both fluorescein and DCJTB in the stamped layer and give forth green and red emissions. As the band gap of DCJTB is around $2.01 \mathrm{eV}$, the green-yellow lights from fluorescein have enough energy to excite DCJTB and act as a sensitizer to increase the efficiency of downshifting in DCJTB. Different from the case of F8BT and DCJTB in Chapter 3, the lowest unoccupied molecular orbital (LUMO) energy level of fluorescein is lower than that of DCJTB and thus direct charge transfer is negligible from fluorescein to DCJTB [159]. Thus the yellow emission is stronger in this experiment compared with the drop casting case in Chapter 3. 


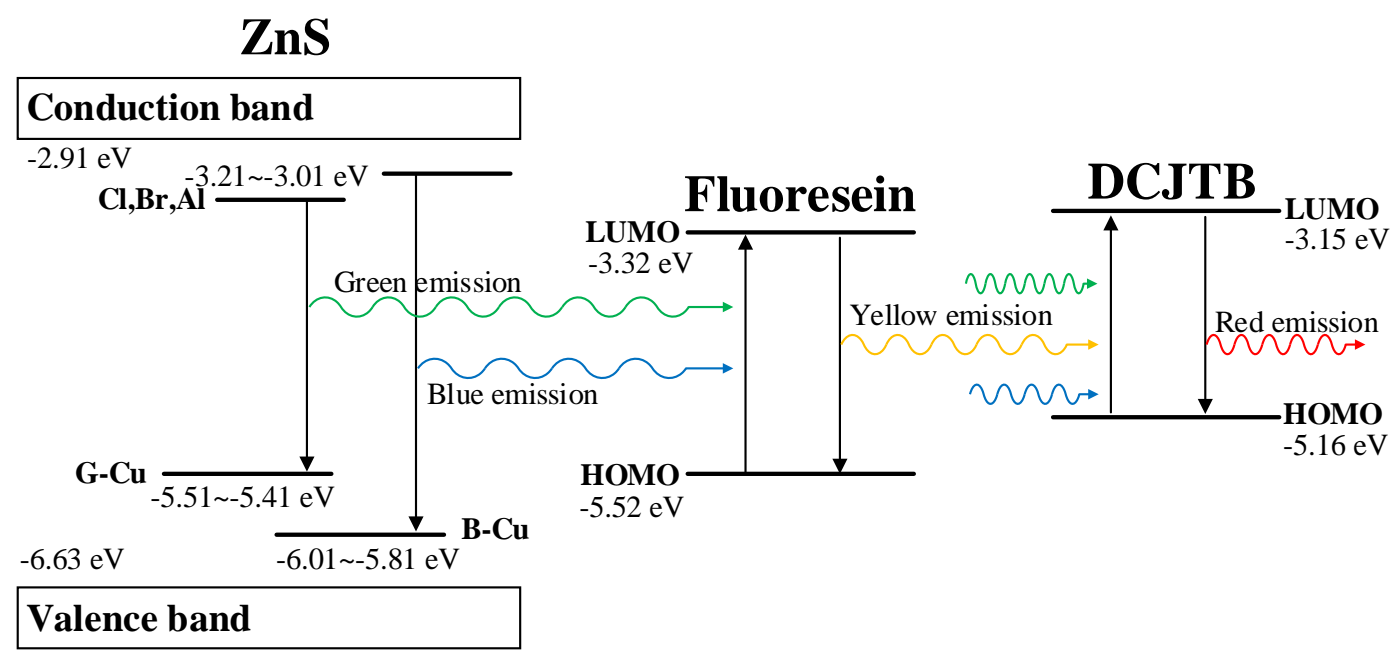

Figure 4.6: Downshifting mechanism of DCJTB and fluorescein from blue ZnS phosphors.

The colors of these hybrid devices with different mass ratios are shown in Figure 4.7. CIE shifts towards the blue region with the increase of operating frequency in all conditions, which agrees well with previous observed frequency dependent performances of ACPEL devices $[45,158]$ and is explained by the model we proposed in Chapter 5. In Figure 4.7, increasing the fluorescein proportion shifts the hybrid device towards white color at 110 $\mathrm{V}$ and $400 \mathrm{~Hz}$, which agrees well with the increased intensity of green and red emissions observed in Figure 4.5. However, the color is still far from white.

\section{Effect of dye concentration}

To study the effect of dye concentration, mass ratio of 1:1 was used as it gives moderate color output as suggested in Figure 4.7. Although CIE of mass ratio 1:7 is closer to white, the small amount of DCJTB may cause the lack of red component when the dye layer thickness increases. To wet stamp a thicker dye layer, there are two possible ways: decreasing $w$ or $t_{d}$. By reducing $w$, less dye will be pressed to the edge while the amount of dyes available for wet stamping is increased with smaller $t_{d}$. Figure 4.8(a) shows the EL spectra with the change of $t_{d}$ to $10 \mathrm{~s}$ and $w$ to $0.40 \mathrm{~kg}$ while other conditions are fixed. With $t_{d}=10$ 


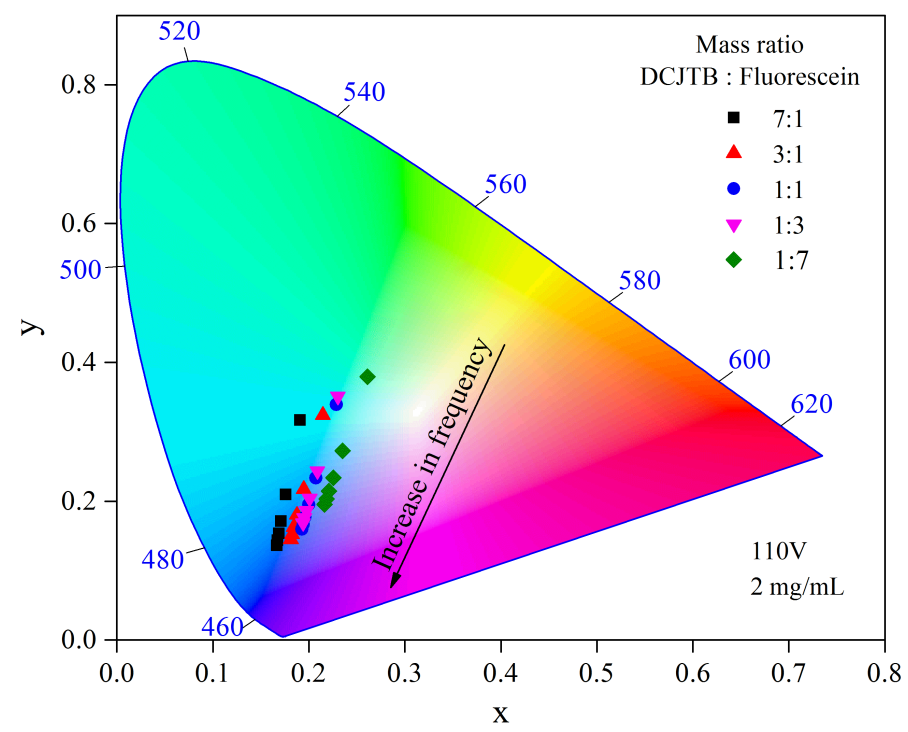

Figure 4.7: CIE at $110 \mathrm{~V}$ and 100, 400, 800, 1200, 1600, $2000 \mathrm{~Hz}$ for hybrid EL films with fixed dye concentration at $2 \mathrm{mg} / \mathrm{mL}$ and different DCJTB:fluorescein mass ratios.

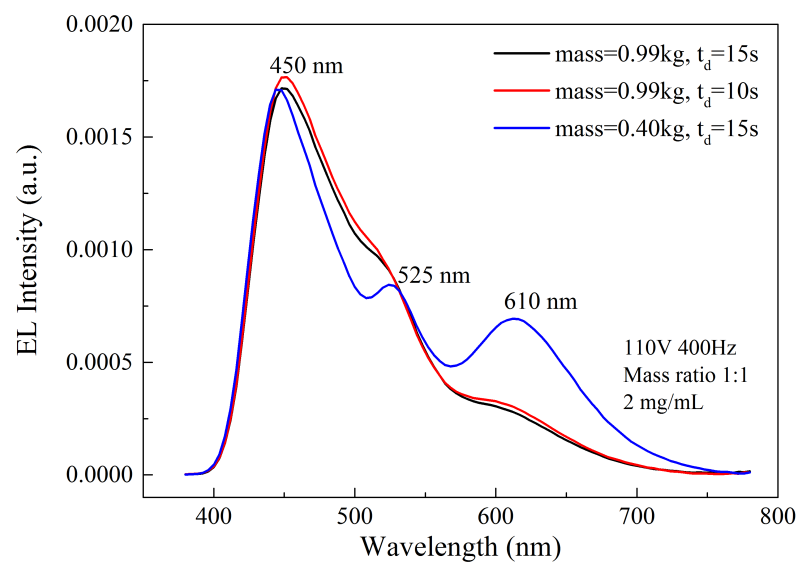

(a)

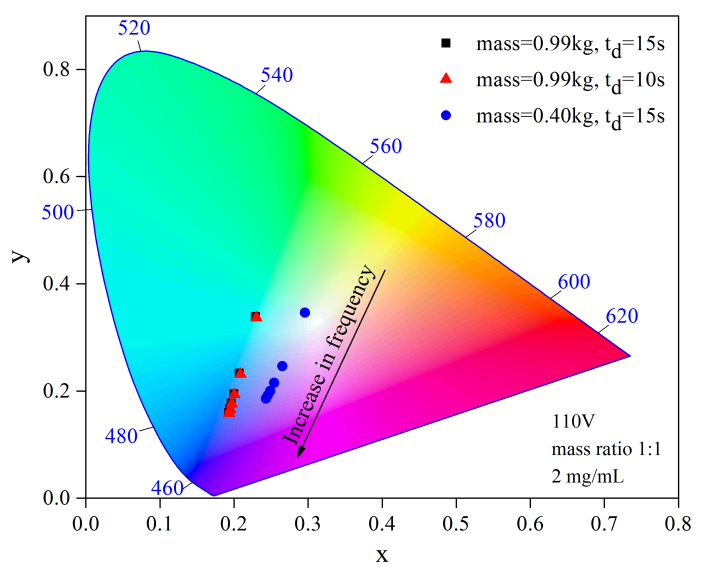

(b)

Figure 4.8: (a) EL spectra at $110 \mathrm{~V}$ and $400 \mathrm{~Hz}$ and (b) CIE at $110 \mathrm{~V}$ and 100, 400, 800, $1200,1600,2000 \mathrm{~Hz}$ for hybrid EL films with weight of $0.40 \mathrm{~kg}$ or $0.99 \mathrm{~kg}$ and drying time of $10 \mathrm{~s}$ or $15 \mathrm{~s}$.

$\mathrm{s}$, the changes of green and red shoulders are minimal while the shoulders become two peaks at the wavelengths of $525 \mathrm{~nm}$ and $610 \mathrm{~nm}$ with $0.40 \mathrm{~kg}$. CIE in Figure 4.8(b) further sees no improvement in color for $t_{d}=10 \mathrm{~s}$ but a big shift towards white color for $w=0.40 \mathrm{~kg}$. Although CIE for $w=0.40 \mathrm{~kg}$ operated at $110 \mathrm{~V}$ and $100 \mathrm{~Hz}$ is $(0.296,0.346)$ and close to white color, the luminance is low $\left(21.63 \mathrm{~cd} / \mathrm{m}^{2}\right)$. 
The efficiency of ACPEL devices is the highest at $400 \mathrm{~Hz}$ [160]. Furthermore, increasing voltage does not alter CIE. Therefore, if we can obtain white color at $400 \mathrm{~Hz}$, luminance can be tuned by changing the operating voltage. The effects of dye concentration on the hybrid device performance with a mass ratio of 1:1 are shown in Figure 4.9. As the dye concentration increases from $2 \mathrm{mg} / \mathrm{mL}$ to $6 \mathrm{mg} / \mathrm{mL}$, three peaks at the wavelengths of 450 $\mathrm{nm}, 540 \mathrm{~nm}$ and $610 \mathrm{~nm}$ respectively start to form and the latter two emissions get intensified. This is due to the increased quantity of both fluorescein and DCJTB downshifting dyes which leads to stronger $540 \mathrm{~nm}$ and $610 \mathrm{~nm}$ emissions. The $450 \mathrm{~nm}$ peaks also experience a little blue shift like that in Figure 4.5. Consequently, the colors of these hybrid devices shift to white with an increased dye concentration as indicated in Figure 4.9(b). A white color with CIE of $(0.304,0.294)$ is achieved for the concentration of $5 \mathrm{mg} / \mathrm{mL}$ and operated at $110 \mathrm{~V}$ and $400 \mathrm{~Hz}$. The image of the stamped hybrid EL films operated at $200 \mathrm{~V}$ and 400 $\mathrm{Hz}$ is shown in the inset of Figure 4.9(b). The white emission has the luminance and CCT uniformities of $95.07 \%$ and $88.56 \%$ respectively and a high luminance of $93.9 \mathrm{~cd} / \mathrm{m}^{2}$ at 200 $\mathrm{V}$ and $400 \mathrm{~Hz}$. The CCT at $200 \mathrm{~V}$ and $400 \mathrm{~Hz}$ is $7162 \mathrm{~K}$ with the CRI of 89.5. However, the luminous efficacy of this stamped white film is only $1.31 \mathrm{~lm} / \mathrm{W}$, which is slightly lower than that of reversed blue EL films. This indicates that there is an energy loss during the downshifting process. Additionally, a 'NTU' pattern was coated on the hybrid device as shown in the inset of Figure 4.9(b). The pattern displays a good uniform white color with blue background from the $\mathrm{ZnS}$ phosphors. This demonstrates the capability of controlling pattern shapes using this wet stamping process.

Warm white hybrid EL films by wet stamping

As shown in previous sections, one single layer of the DCJTB-fluorescein coating can only produce white color by the wet stamping process. To obtain warm white color, there should be enough red component in the EL emission of the hybrid film. Therefore, multiple layers of DCJTB were wet-stamped onto reversed green EL films. In Figure 4.10(a), as the 


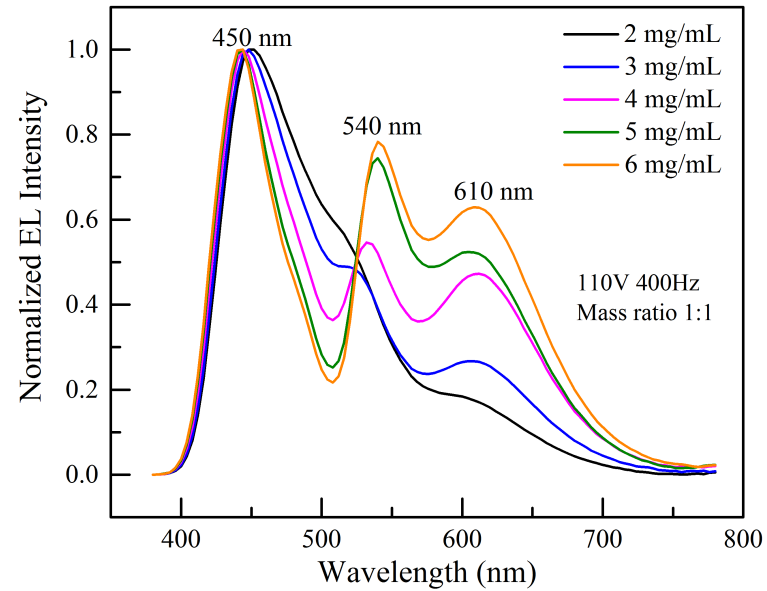

(a)

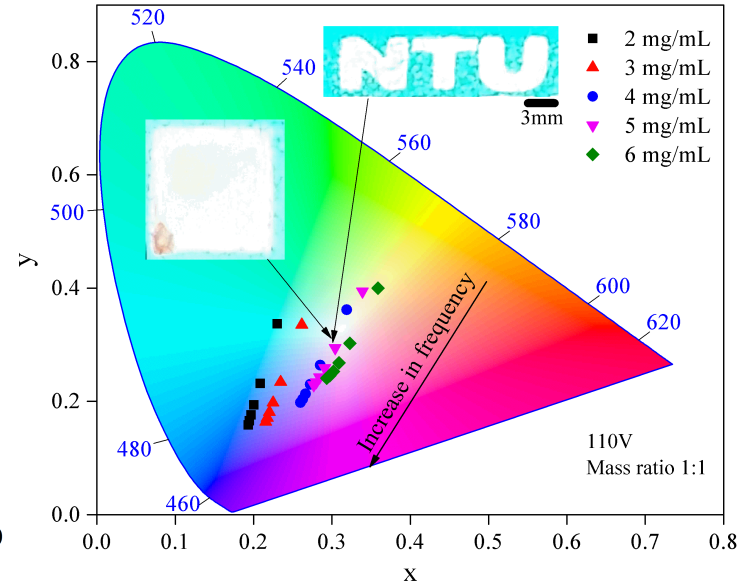

(b)

Figure 4.9: (a) EL spectra at $110 \mathrm{~V}$ and $400 \mathrm{~Hz}$ and (b) CIE at $110 \mathrm{~V}$ and 100, 400, 800, 1200, 1600, $2000 \mathrm{~Hz}$ for hybrid EL films with fixed DCJTB:fluorescein mass ratio at 1:1 and varied dye concentrations

number of DCJTB layers increases, the red peak intensity increases while the $500 \mathrm{~nm}$ intensity decreases. This suggests thicker DCJTB coating is achieved with more layers, leading to more downshifting phenomenon. However, the red peak is not high enough to produce warm white color. As shown in Fig. 4.10(b), the lowest CCT is only 4344 $\mathrm{K}$ for the hybrid films coated with five layers of DCJTB and operated at $200 \mathrm{~V}$ and 400 Hz. Continuous increasing of the number of DCJTB layers may further reduce the CCT. However, from Figure 4.3(a), DCJTB is not effectively excited by the green $\mathrm{ZnS}$ phosphors but more effectively by green-yellow emissions from fluorescein. Therefore, the mixture of DCJTB-fluorescein was coated subsequently.

A $4 \mathrm{mg} / \mathrm{mL}$ of DCJTB-fluorescein solution with the mass ratio of 1:1 was formulated and multiple layers were wet stamped over reversed green EL films. With the increased number of layers, the $628 \mathrm{~nm}$ intensity increases first until five layers and decreases from six layers as shown in Figure 4.11(a). However, the $496 \mathrm{~nm}$ and $524 \mathrm{~nm}$ peaks do not follow a trend. The increase of the $628 \mathrm{~nm}$ peak intensity is due to the increased amount of DCJTB along with the increased coating thickness while the reduced intensity at more layers may be due to the significant scattering within the coating layer. Consequently, CCT 


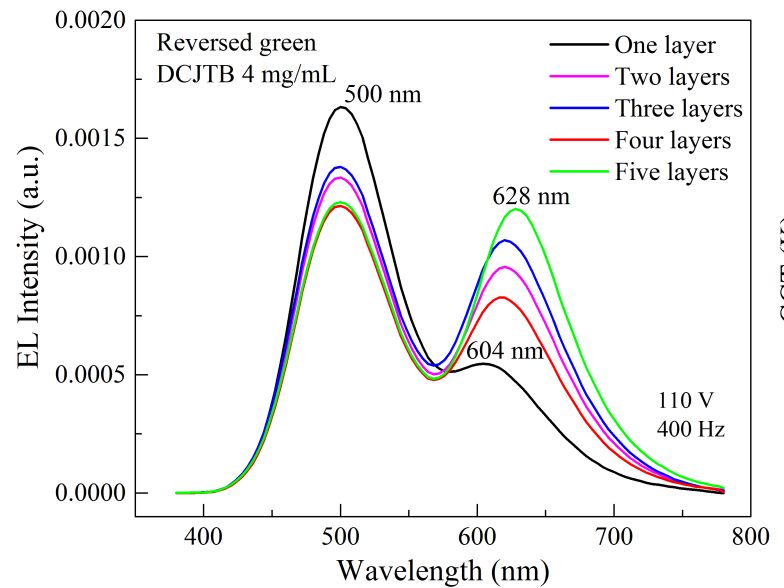

(a)

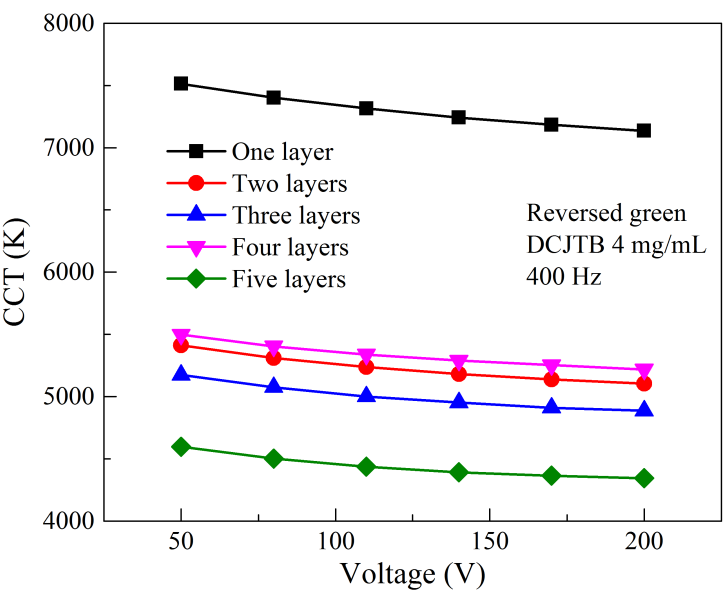

(b)

Figure 4.10: (a) EL spectra at $110 \mathrm{~V}$ and $400 \mathrm{~Hz}$ and (b) CCT as a function of voltage at $400 \mathrm{~Hz}$ for hybrid EL films stamped with one to five layers of DCJTB solution at $4 \mathrm{mg} / \mathrm{mL}$.

at six layers is higher than that at five layers as illustrated in Figure 4.10(b). CCT decreases with the increased number of layers when less than five layers are coated. The five-layer hybrid EL film produces a warm white color as shown in the inset of Fig. 4.11(b) with the luminance of $89.3 \mathrm{~cd} / \mathrm{m}^{2}$ operated at $200 \mathrm{~V}$ and $400 \mathrm{~Hz}$. This warm white color has a CCT of $2864 \mathrm{~K}$ with a CRI of 86.2 while the luminance and CCT uniformities are $93 \%$ and $93.03 \%$ respectively. Visually, the warm white color appears to be a bit yellowish due to the large amount of green component from fluorescein. However, it is noticed that there are dye stains over the stamped surface and the border of stamped surface is thicker with multiple lines due to the inaccurate alignment during the multilayer wet stamping process. Therefore, although warm white color is demonstrated by wet stamping process, this technique is not suitable for large scale and commercial applications. Moreover, the luminous efficacy is also low at $1.45 \mathrm{~lm} / \mathrm{W}$.

\subsubsection{Optimization of bar coating conditions}

The need for multilayer structure of wet-stamped coating is primarily due to the insufficient solubility of DCJTB in ethanol. To overcome this issue, a similar red dye DCM2 was used 


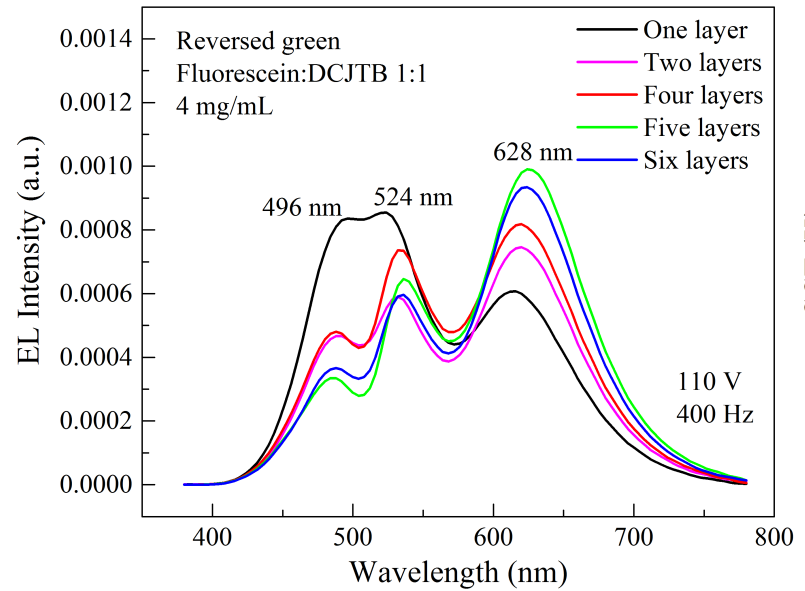

(a)

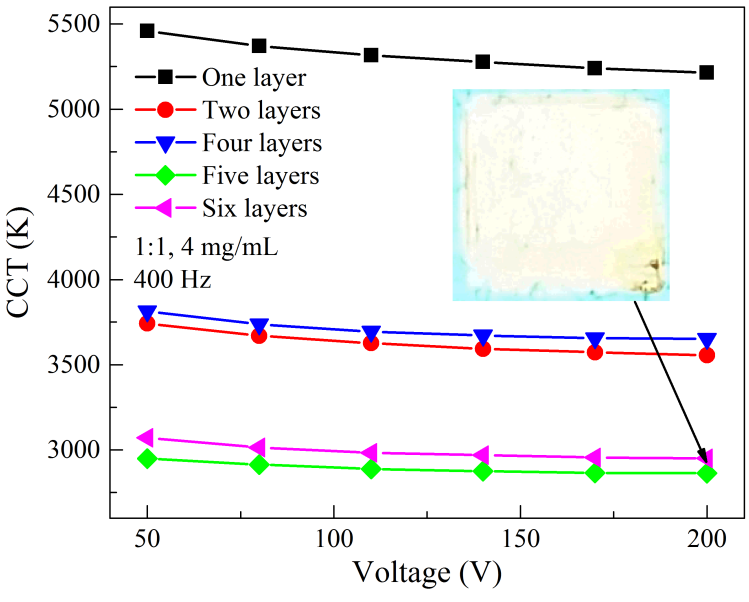

(b)

Figure 4.11: (a) EL spectra at $110 \mathrm{~V}$ and $400 \mathrm{~Hz}$ and (b) CCT as a function of voltage at 400 $\mathrm{Hz}$ for hybrid EL films stamped with one, two, four and five layers of DCJTB-fluorescein solution at $4 \mathrm{mg} / \mathrm{mL}$ and with the mass ratio of $1: 1$. Inset: the image showing warm white color of stamped sample operated at $200 \mathrm{~V}$ and $400 \mathrm{~Hz}$.

and the PL and absorbance spectra are shown in Figure 4.12. When excited by $450 \mathrm{~nm}$ photons, DCM2-ethanol solution has a red emission with the peak wavelength of $664 \mathrm{~nm}$. The absorbance spectrum exhibits a peak wavelength of $509 \mathrm{~nm}$, which demonstrates that DCM2 can be effectively excited by the green $\mathrm{ZnS}$ phosphors with the peak wavelength of around $500 \mathrm{~nm}$. Primarily, it is necessary to determine the best coating conditions to obtain the best uniformity and lowest CCT.

\section{Bar thread thickness}

The bar-coated coating thickness is mostly determined by the thickness of bar thread. In Figure 4.13(a), with the printing speed fixed at $100 \mathrm{~mm} / \mathrm{s}$, the increase of thread thickness causes the $613 \mathrm{~nm}$ intensity to increase when the thickness is less than $80 \mu \mathrm{m}$, after which the intensity drops. This may be due to the luminescence quenching with thicker coating layers or aggregations. The $492 \mathrm{~nm}$ and $530 \mathrm{~nm}$ peak intensities exhibit inverse effects with the $613 \mathrm{~nm}$ emission. The change of $613 \mathrm{~nm}$ peak intensity is also reflected in the CIE map of these hybrid films with different thread thicknesses at different operating frequencies as 


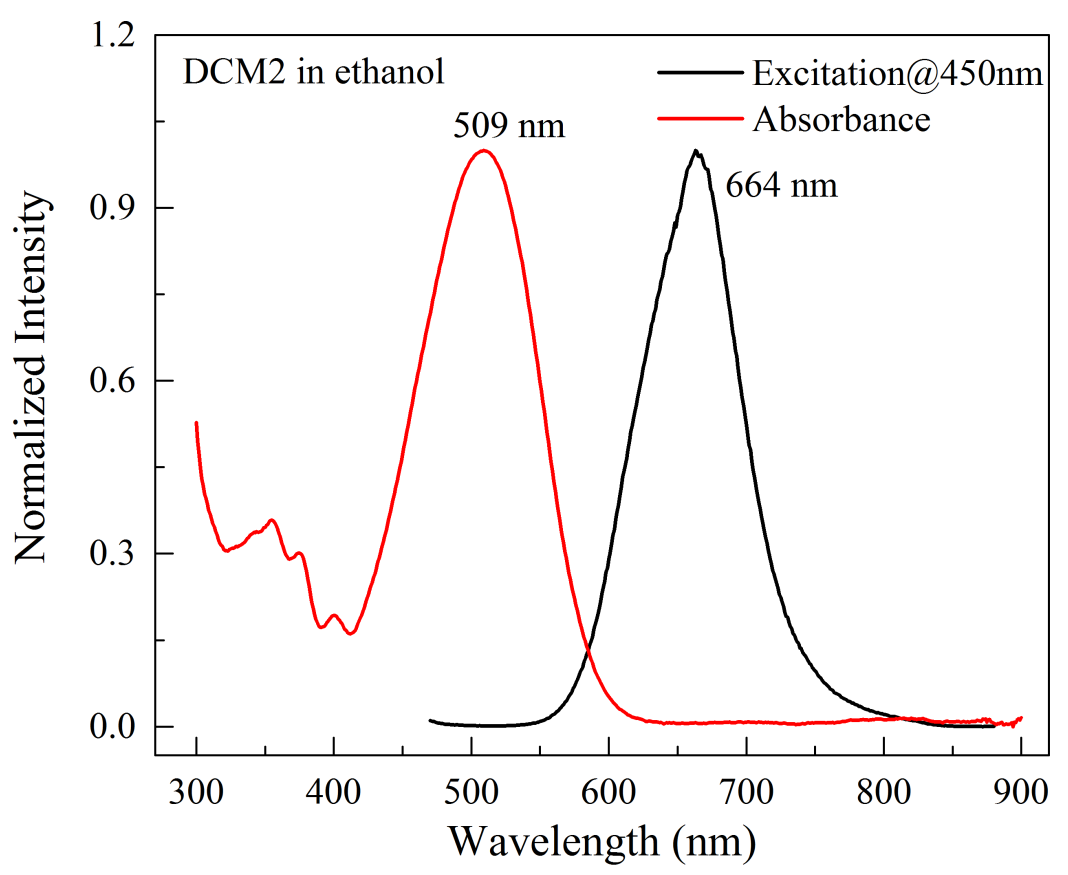

Figure 4.12: PL emission spectrum excited by $450 \mathrm{~nm}$ photons and absorbance spectrum of DCM2 dissolved in ethanol.

shown in Figure 4.13(b). When coated with the tread thickness of $80 \mu \mathrm{m}$, the hybrid film is closest to the red color. The color quenching is also observed with the thickness of 100 and $120 \mu \mathrm{m}$. These hybrid films are still within the green color region, which suggests an insufficient $613 \mathrm{~nm}$ intensity.

The resulted CCT with the consideration of the effects of the thread thickness is shown in Figure 4.14(a) at different frequencies. The hybrid films coated by the bar with the thread thickness of $80 \mu \mathrm{m}$ again have the lowest CCT values at all frequencies. However, CCT is still above $6000 \mathrm{~K}$, which is not close to the warm white color at around $3000 \mathrm{~K}$. The depreciation of CCT is also observed at thicker thread. In Figure 4.14(b), the $80 \mu \mathrm{m}$ hybrid film has the highest luminance uniformity at $95.2 \%$ but its CCT uniformity is the lowest at 90.7\%. With the CCT as our primary factor, bar with the thread thickness of $80 \mu \mathrm{m}$ is used for the bar coating process in all our future experiments. 


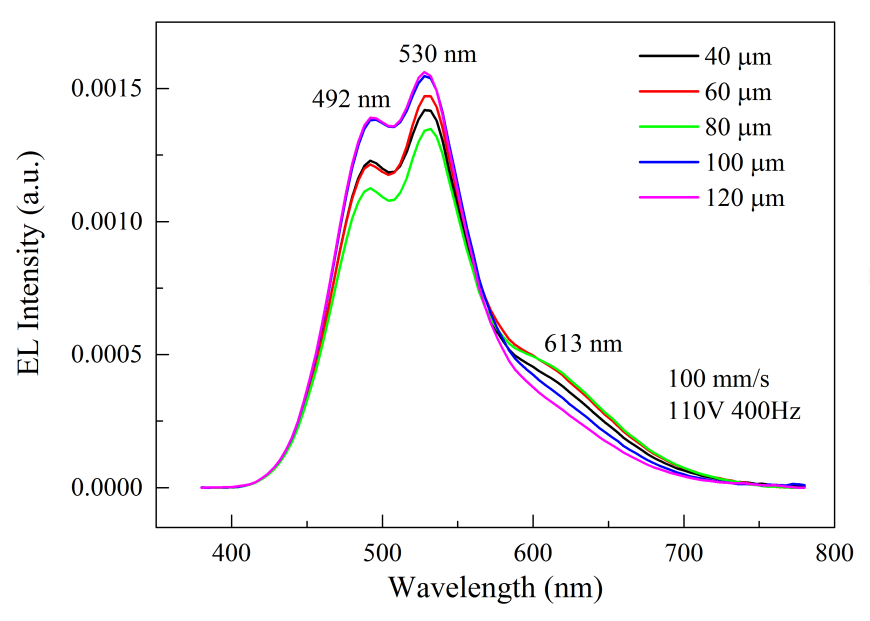

(a)

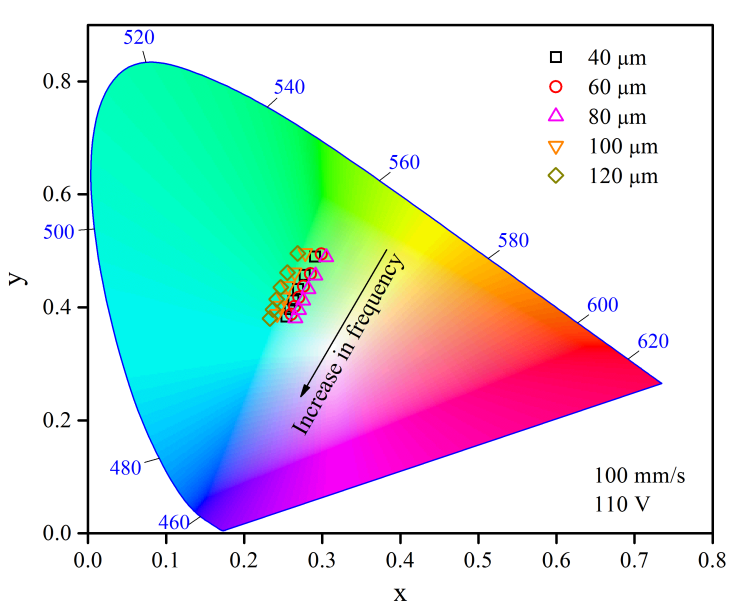

(b)

Figure 4.13: (a) EL spectra at $110 \mathrm{~V}$ and $400 \mathrm{~Hz}$ and (b) CIE at $110 \mathrm{~V}$ and 100, 400, 800, $1200,1600,2000 \mathrm{~Hz}$ for bar coated hybrid EL films using bars with thread thicknesses of $40,60,80,100$, and $120 \mu \mathrm{m}$ and speed of $100 \mathrm{~mm} / \mathrm{s}$.

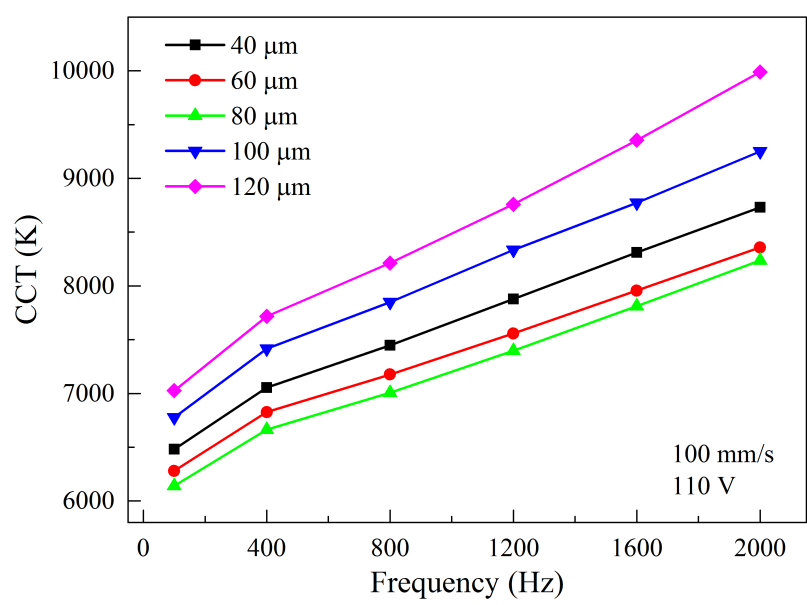

(a)

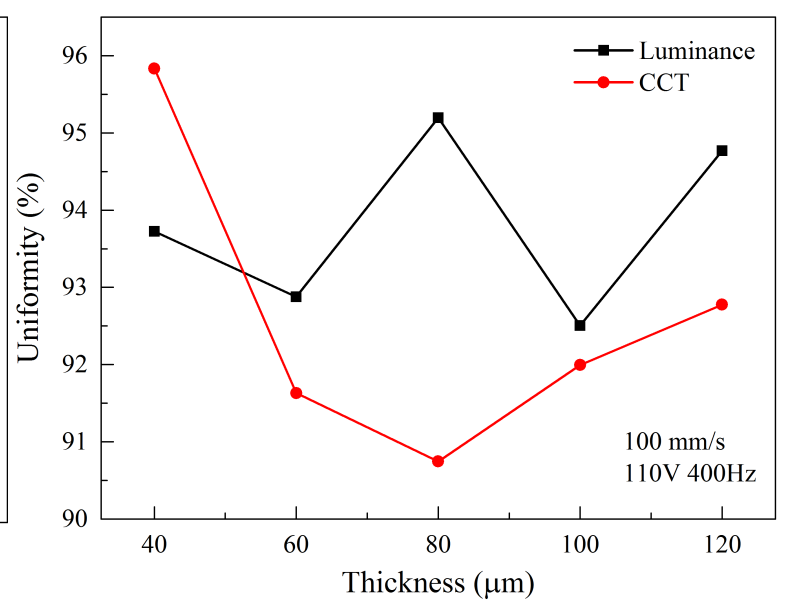

(b)

Figure 4.14: (a) CCT at $110 \mathrm{~V}$ as a function of frequency and (b) uniformity at $110 \mathrm{~V}$ and $400 \mathrm{~Hz}$ for bar coated hybrid EL films using bars with thread thicknesses of 40,60, 80, 100 , and $120 \mu \mathrm{m}$ and speed of $100 \mathrm{~mm} / \mathrm{s}$.

\section{Printing speed}

Printing speed is also another major factor in determining the quality of bar-coated hybrid films. As shown in Figure 4.15(a), increasing the printing speed from $10 \mathrm{~mm} / \mathrm{s}$ to $100 \mathrm{~mm} / \mathrm{s}$ enhances the red emission and reduces both $492 \mathrm{~nm}$ and $528 \mathrm{~nm}$ peak intensities. This is 
due to the fact that at a high printing speed the dye solution does not have enough time to escape from the two ends of the bars. Consequently more dyes are printed leading to more downshifted red emissions from DCM2. It is noticed that the red component intensities with the printing speed of 50 and $100 \mathrm{~mm} / \mathrm{s}$ do not differ much. This is also seen in Figure 4.15(b), where CIEs of both hybrid films are close to each other. The increase of the printing speed shifts CIE toward red color. As shown in Figure 4.16(a), CCT decreases with the printing speed so as the luminance and CCT uniformity in Figure 4.16(b). To obtain a lower CCT without sacrificing too much luminance and CCT uniformity, the printing speed of $50 \mathrm{~mm} / \mathrm{s}$ is used for future bar coating experiments.

\subsubsection{Bar coated hybrid films}

\section{Effect of solution concentration}

As DCM2 can be effectively excited by green $\mathrm{ZnS}$ phosphors, only DCM2 is coated over reversed green EL films to obtain warm white color. The EL spectra of hybrid EL films with only one layer of DCM2 solution with the concentrations of $1.0,2.0$ and $4.0 \mathrm{mg} / \mathrm{mL}$ are shown in Figure 4.17(a). Increasing the concentration enhances the $608 \mathrm{~nm}$ peak and reduces the $500 \mathrm{~nm}$ peak intensity. This is due to the higher amount of coated DCM2, which also shifts CIE toward red color as shown in Figure 4.17(b). In Figure 4.18(a), CCT is also reduced with the increase of dye concentration. However, both luminance and CCT uniformities reduce with the dye concentration.

\section{Effect of number of coated layers}

The CCT of one layer of $4.0 \mathrm{mg} / \mathrm{mL}$ DCM2 solution is still above $6000 \mathrm{~K}$, which suggests insufficient red emissions. To increase the red component, more layers are printed and the EL spectra are shown in Figure 4.19(a). With the increased number of layers, the proportion of $620 \mathrm{~nm}$ emission over the $500 \mathrm{~nm}$ emission increases, although the absolute amount of $620 \mathrm{~nm}$ peak intensity of five layers is smaller than that of two layers. This is probably due 


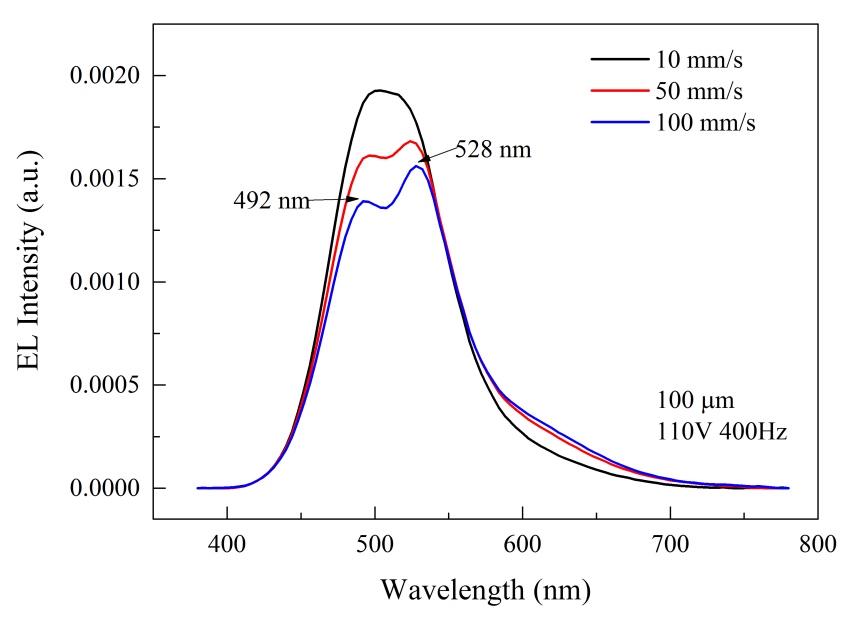

(a)

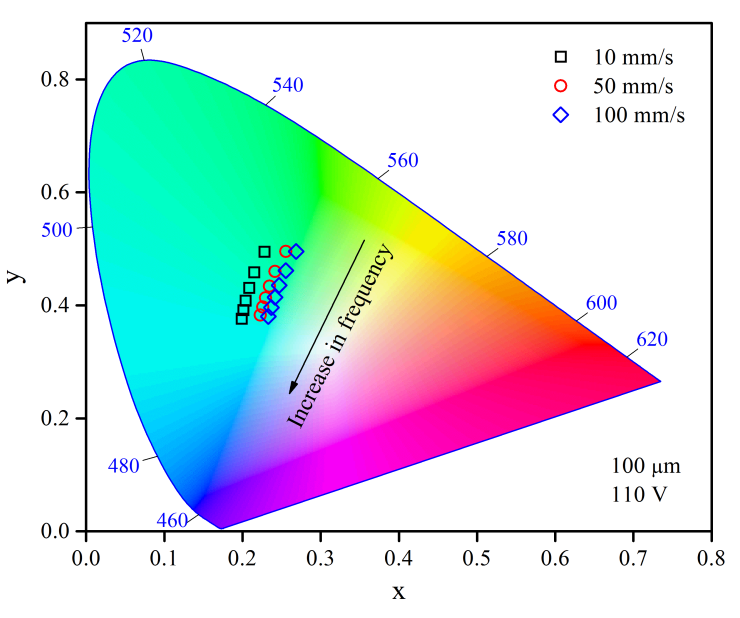

(b)

Figure 4.15: (a) EL spectra at $110 \mathrm{~V}$ and $400 \mathrm{~Hz}$ and (b) CIE at $110 \mathrm{~V}$ and 100, 400, 800, $1200,1600,2000 \mathrm{~Hz}$ for bar coated hybrid EL films using bars with thread thicknesses of $120 \mu \mathrm{m}$ and speed of 10,50 and $100 \mathrm{~mm} / \mathrm{s}$.

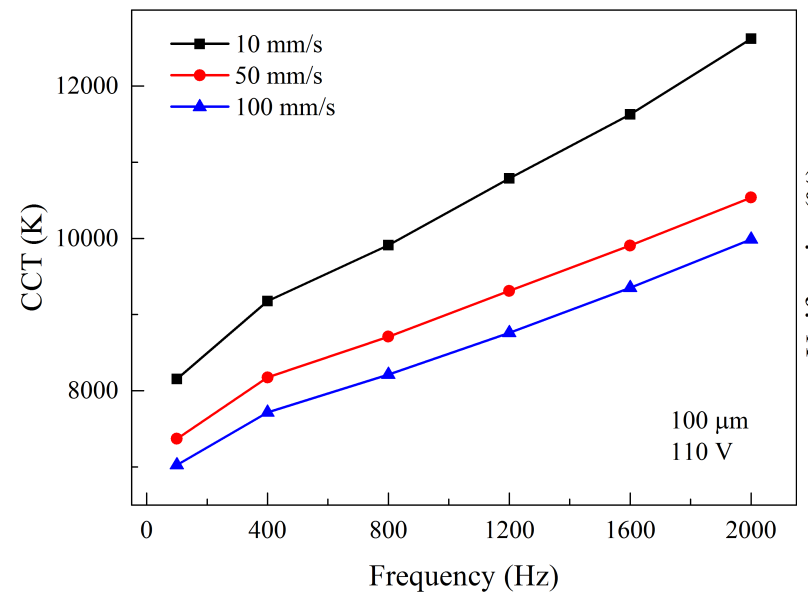

(a)

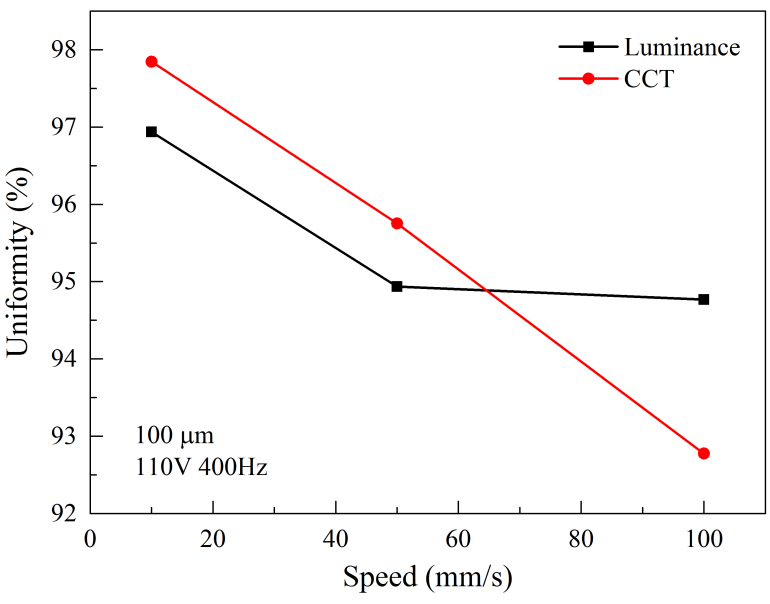

(b)

Figure 4.16: (a) CCT at $110 \mathrm{~V}$ as a function of frequency and (b) uniformity at $110 \mathrm{~V}$ and $400 \mathrm{~Hz}$ for bar coated hybrid EL films using bars with thread thicknesses of $120 \mu \mathrm{m}$ and speed of 10,50 and $100 \mathrm{~mm} / \mathrm{s}$.

to the sample-sample variation. The increased number of layers also shifts CIE toward red color as shown in Figure 4.19(b). It is noted that CIE of five layers of DCM2 solution is located within the red region. In Figure 4.20(a), CCT is reduced further with the increased number of layers and a CCT of $5385 \mathrm{~K}$ is obtained with five layers of coatings. With the increased number of layers, the CCT uniformity is also increased but luminance uniformity 


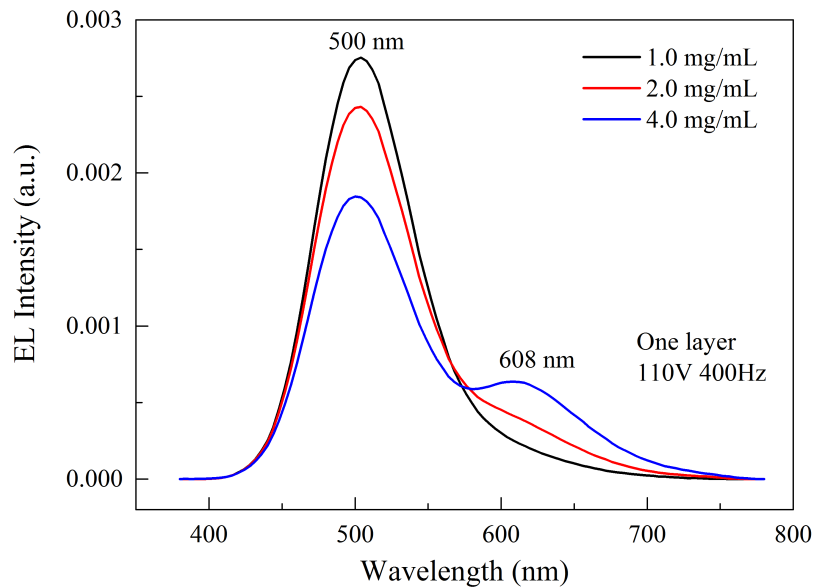

(a)

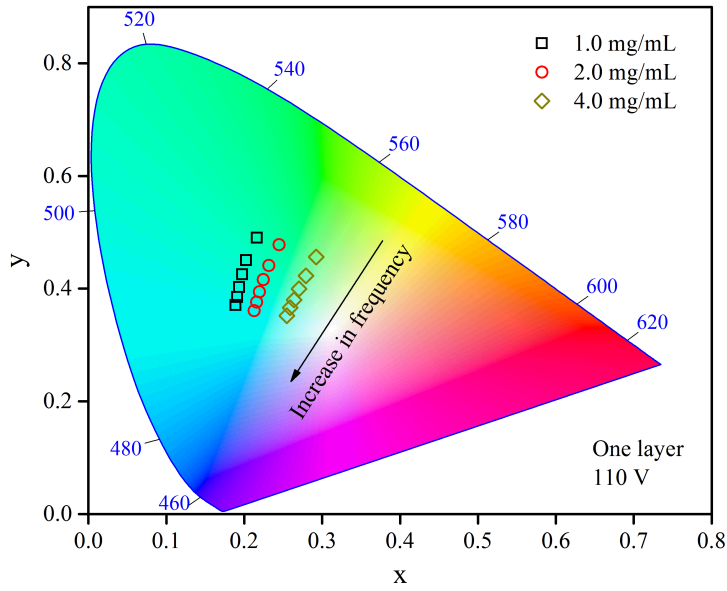

(b)

Figure 4.17: (a) EL spectra at $110 \mathrm{~V}$ and $400 \mathrm{~Hz}$ and (b) CIE at $110 \mathrm{~V}$ and 100, 400, 800, $1200,1600,2000 \mathrm{~Hz}$ for hybrid EL films with one layer of bar-coated DCM2 solution at $1.0,2.0$ and $4.0 \mathrm{mg} / \mathrm{mL}$.

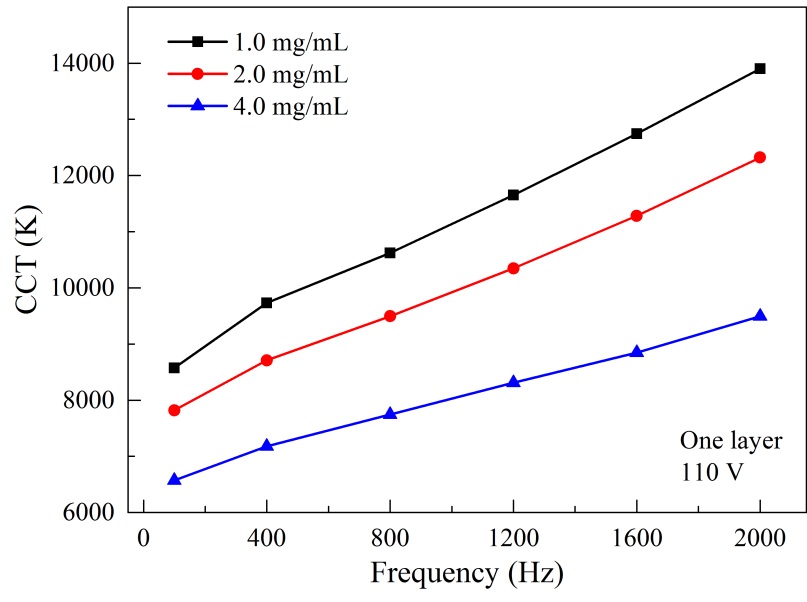

(a)

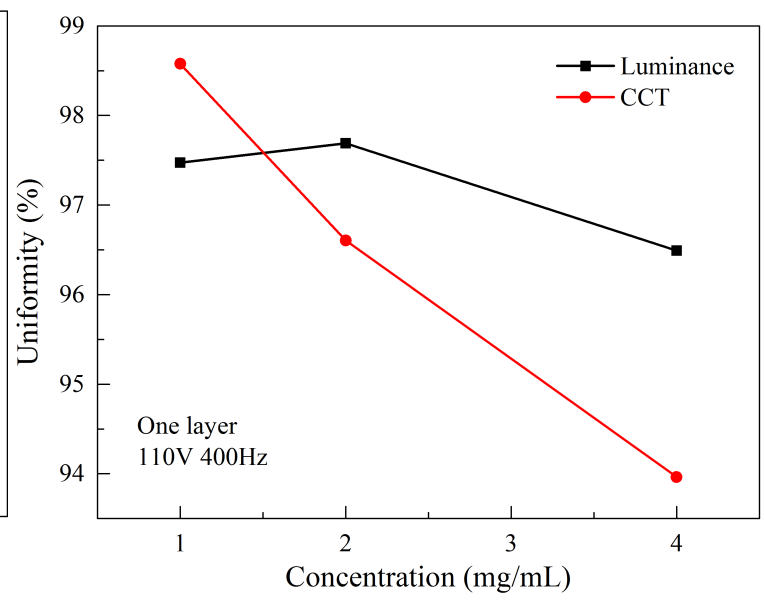

(b)

Figure 4.18: (a) CCT at $110 \mathrm{~V}$ and 100, 400, 800, 1200, 1600, $2000 \mathrm{~Hz}$ and (b) uniformity at $110 \mathrm{~V}$ and $400 \mathrm{~Hz}$ for hybrid EL films with one layer of bar-coated DCM2 solution at $1.0,2.0$ and $4.0 \mathrm{mg} / \mathrm{mL}$.

is the lowest with two layers of coatings.

\section{Warm white hybrid films}

From above experiments, increasing the concentrations and numbers of coated layers reduces CCT. Therefore, a $15 \mathrm{mg} / \mathrm{mL}$ DCM2-fluorescein solution with the mass ratio of 9:1 


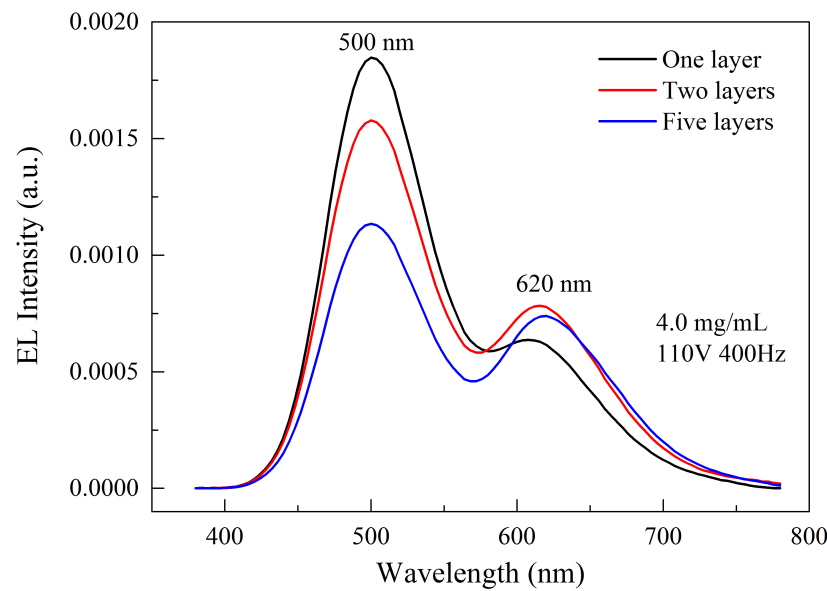

(a)

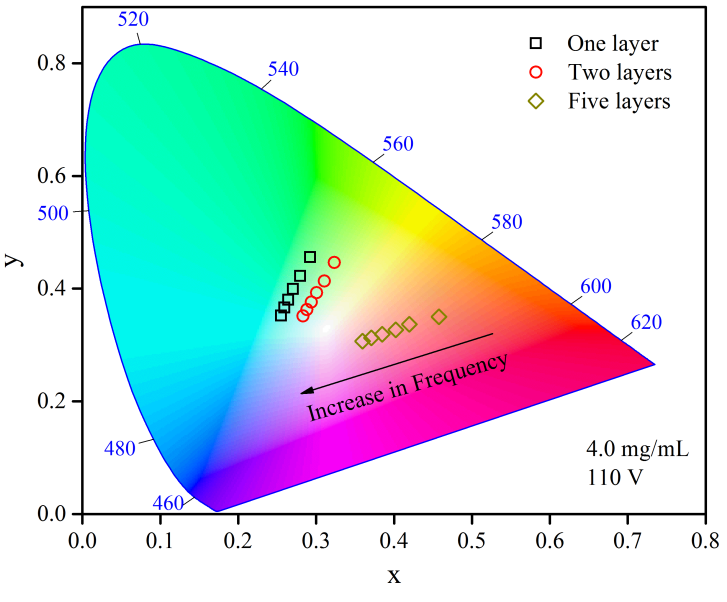

(b)

Figure 4.19: (a) EL spectra at $110 \mathrm{~V}$ and $400 \mathrm{~Hz}$ and (b) CIE at $110 \mathrm{~V}$ and 100, 400, 800, $1200,1600,2000 \mathrm{~Hz}$ for hybrid EL films with one, two and five layer of bar-coated DCM2 solution at $4.0 \mathrm{mg} / \mathrm{mL}$.

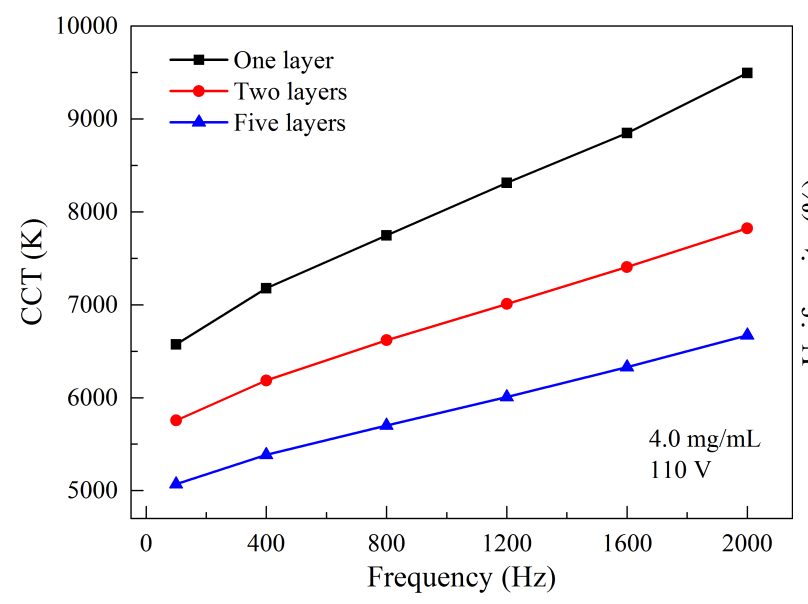

(a)

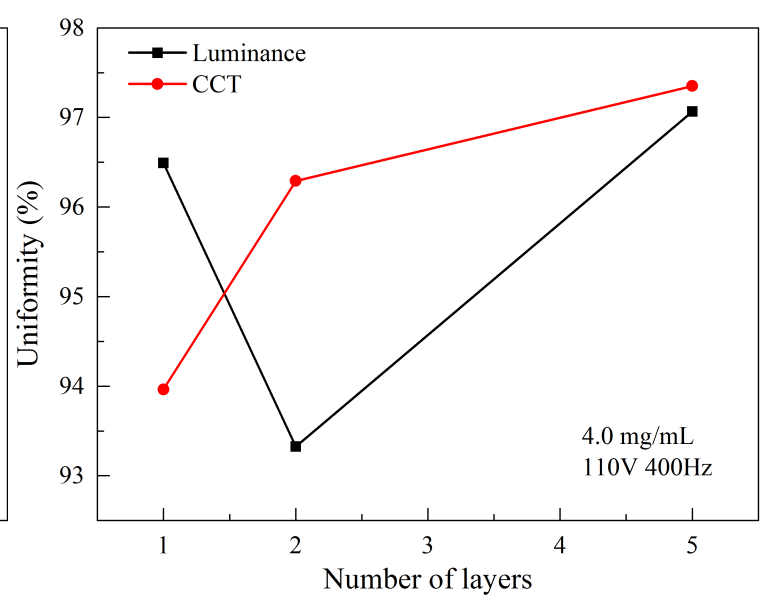

(b)

Figure 4.20: (a) CCT at $110 \mathrm{~V}$ as a function of frequency and (b) uniformity at $110 \mathrm{~V}$ and $400 \mathrm{~Hz}$ for hybrid EL films with one, two and five layers of bar-coated DCM2 solution at $4.0 \mathrm{mg} / \mathrm{mL}$.

is bar coated over reversed green EL films. The $612 \mathrm{~nm}$ red component of the EL emission is not improved compared with that in Figure 4.19(a). CIE in Figure 4.21(b) indicates that this hybrid film is still yellow in color. The resulted CCT in Figure 4.22 is more than 5500 $\mathrm{K}$. This suggests that DCM2 is not able to achieve warm white color probably due to the low luminescence intensity or high quenching effect at high concentrations. Consequently, 


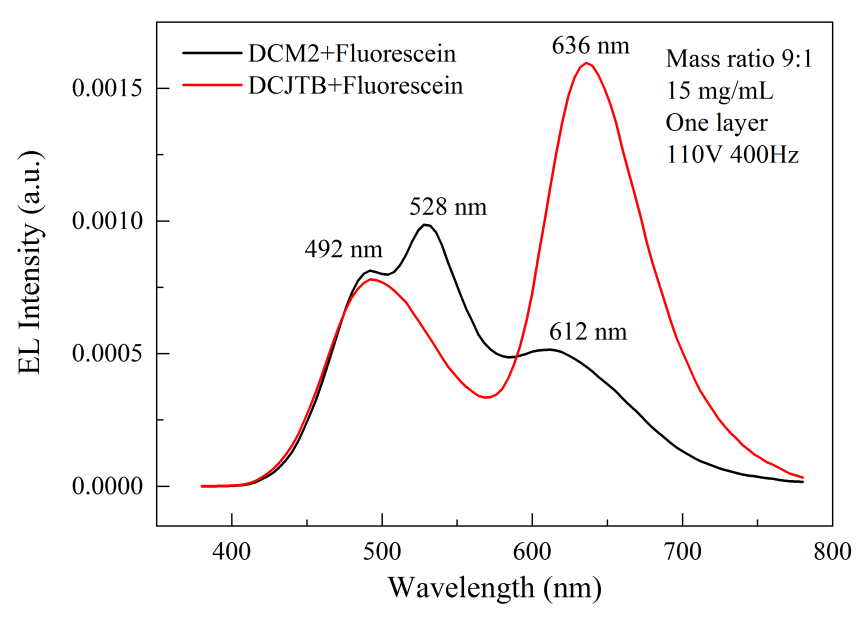

(a)

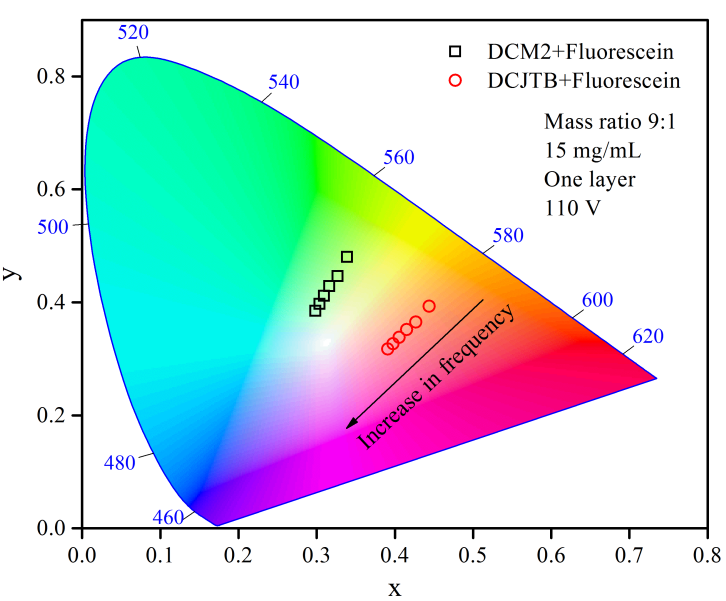

(b)

Figure 4.21: (a) EL spectra at $110 \mathrm{~V}$ and $400 \mathrm{~Hz}$ and (b) CIE at $110 \mathrm{~V}$ as a function of frequency for hybrid EL films with one layer of bar-coated DCM2-fluorescein or DCJTBfluorescein solution at $15.0 \mathrm{mg} / \mathrm{mL}$ and with the mass ratio of 9:1.

DCJTB is used as the red downshifting material. A $15 \mathrm{mg} / \mathrm{mL}$ DCJTB-fluorescein solution with the mass ratio of 9:1 is prepared using the same method as DCM2-fluorescein solution. The hybrid film with only one layer of such solution coated exhibits two peaks with the peak wavelengths of $492 \mathrm{~nm}$ and $636 \mathrm{~nm}$ as shown in Figure 4.21(a). The intensity of the $636 \mathrm{~nm}$ peak is significant enough that CIE of this hybrid film is located in red region in Figure 4.21(b). The resulted CCT operated at $200 \mathrm{~V}$ and $400 \mathrm{~Hz}$ is $2820 \mathrm{~K}$ with the luminance of $72.28 \mathrm{~cd} / \mathrm{m}^{2}$ and the image of warm white hybrid film is shown in the inset of Figure 4.22. The obtained warm white color is a bit reddish due to the significant amount of red emissions. The luminance and CCT uniformities of this film are $93.5 \%$ and $92.1 \%$ respectively. The luminous efficacy of this warm white EL film is the lowest at $0.5 \mathrm{~lm} / \mathrm{W}$ and its CRI is only 44.76.

\subsubsection{Aging study}

The aging behaviors of white EL films hybrid with wet-stamped DCJTB-fluorescein dyes are studied under white light aging and EL aging. Under white light aging, the red peak intensity from stamped white films readily decreases with aging time and almost diminishes 


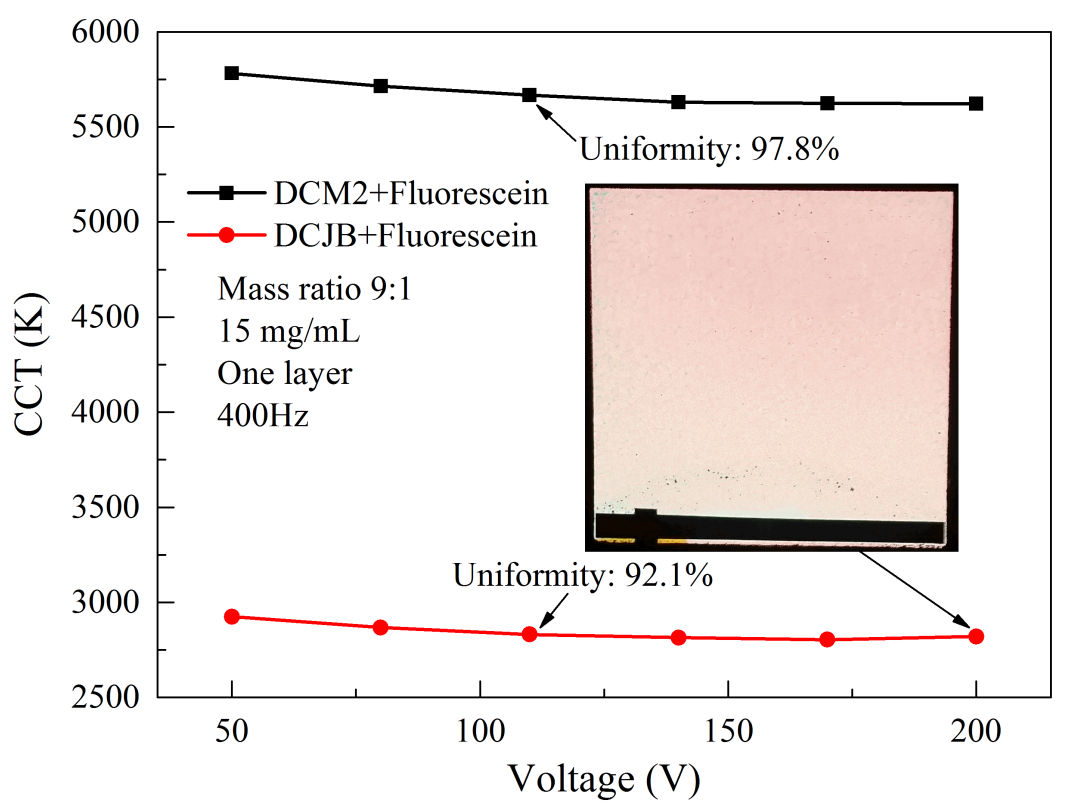

Figure 4.22: CCT as a function of voltage at $400 \mathrm{~Hz}$ for hybrid EL films with one layer of bar-coated DCM2-fluorescein or DCJTB-fluorescein solution at $15.0 \mathrm{mg} / \mathrm{mL}$ and with the mass ratio of 9:1. Inset: warm white EL film bar-coated with DCJTB-fluorescein solution operated at $200 \mathrm{~V}$ and $400 \mathrm{~Hz}$.

when aged for $88.33 \mathrm{hr}$. With less downshifting effects from DCJTB, the peak intensity of dowdnshifted emissions from fluorescein increases. As the white light aging progresses, the green peak disappears. This indicates that fluorescein also degrades under white light conditions but with a slower degradation rate compared to DCJTB. The luminance, however, generally increases with aging time. This is due to the reduced luminous loss during downshifting process when fluorescein and DCJTB degrade. Under EL aging, DCJTB experiences degradation but with a slower rate compared to that under white light aging and the red peak intensity decreases gradually. Fluorescein, however, degrades even slower and its emission curve is only changed to a shoulder from a peak shape from $281.5 \mathrm{hr}$. The luminance generally decreases with time largely due to the degradation of reversed blue EL films as suggested from Figure 3.22(b). 


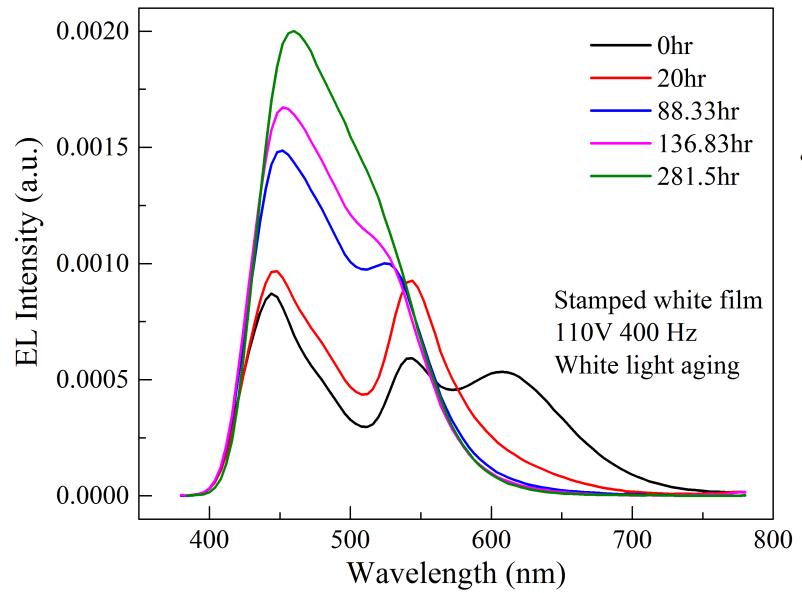

(a)

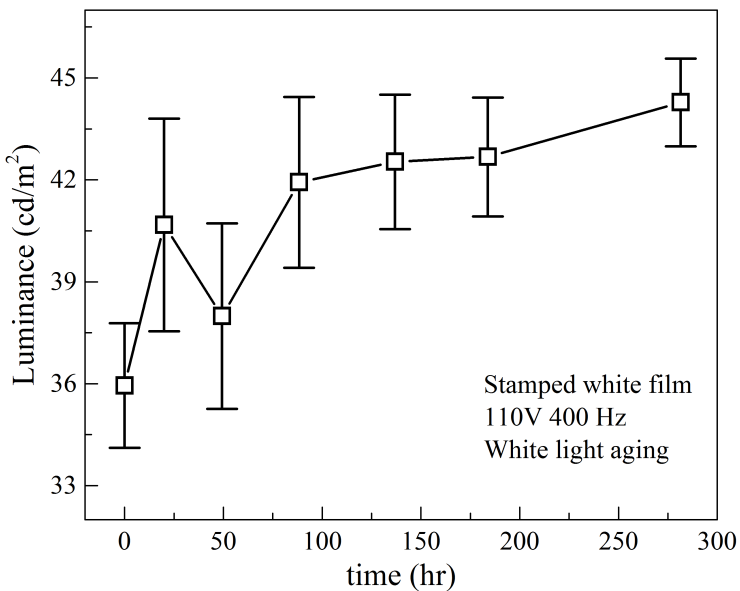

(b)

Figure 4.23: (a) EL spectra and (b) luminance as a function of time for wet-stamped white hybrid EL films with a layer of DCJTB and fluorescein dyes exposed to a constant white light source.

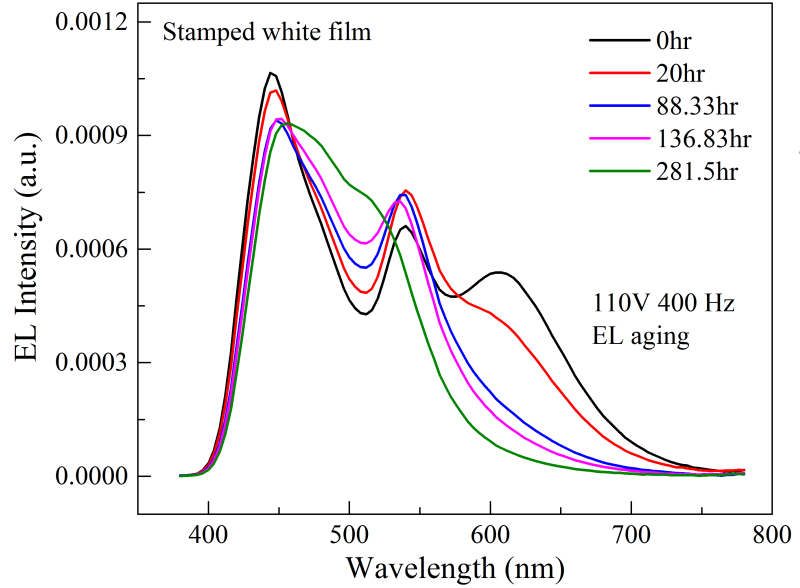

(a)

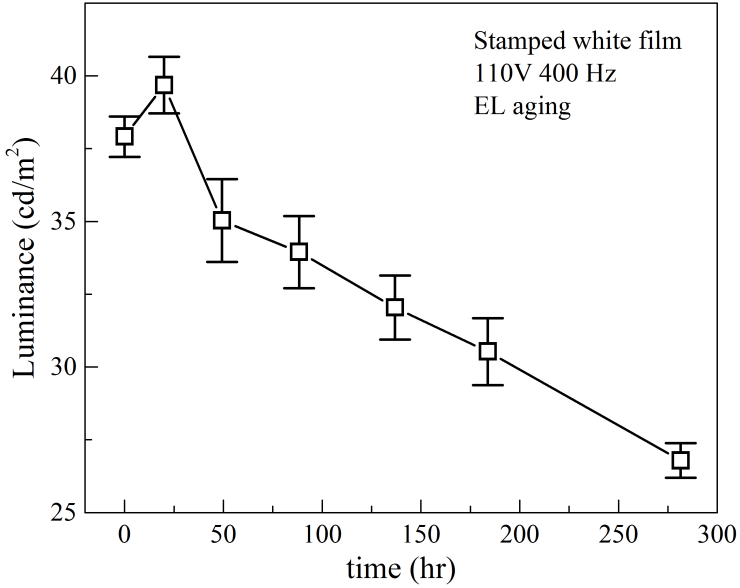

(b)

Figure 4.24: (a) EL spectra and (b) luminance as a function of time for wet-stamped white hybrid EL films with a layer of DCJTB and fluorescein dyes operated at $110 \mathrm{~V}$ and $400 \mathrm{~Hz}$.

\subsection{Conclusions}

DCJTB, DCM2 and fluorescein have been investigated by PL methods and are found to have the capability of downshifting blue emissions from $\mathrm{ZnS}$ phosphors to red and green emissions to obtain white or warm white hybrid EL films. Wet stamping process has been 
implemented to coat a layer of DCJTB-fluorescein solution to fabricated white pattern. Through the design of experimental matrix, the best stamping conditions are found to be $0.99 \mathrm{~kg}$ of the stamping block, $60{ }^{\circ} \mathrm{C}$ of temperature, $15 \mathrm{~s}$ of drying time, and $10 \mathrm{~s}$ of stamping time. With these conditions, the mixed solutions of different DCJTB:fluorescein mass ratios are stamped and the green and red emissions are intensified with the colors shifting toward white with the increase of mass ratio. However, the green and red components are not strong enough for the production of white color due to the small thickness of the dye layer. Reducing the weight to $0.40 \mathrm{~kg}$ increases the coating thickness and the light output. With the modification of dye concentrations, white color with CIE of $(0.304,0.294)$ is achieved with the luminance of $93.9 \mathrm{~cd} / \mathrm{m}^{2}$ at $200 \mathrm{~V}$ and $400 \mathrm{~Hz}$. A white 'NTU' pattern is created to demonstrate the capability and potential of this method for the future development of flexible displays. With the increased number of layers, a warm white square pattern is achieved with CCT of $2864 \mathrm{~K}$. However, the device finish is not satisfactory and not large area. Therefore, a bar coating process is used with bar thread thickness and printing speed optimized to be $80 \mu \mathrm{m}$ and $50 \mathrm{~mm} / \mathrm{s}$. Increasing dye concentration or number of layers reduces CCT. Eventually a CCT of $2820 \mathrm{~K}$ is obtained for the hybrid film bar coated with a layer of $15.0 \mathrm{mg} / \mathrm{mL}$ DCJTB-fluorescein solution with the mass ratio of 9:1. Both DCJTB and fluorescein used in wet stamping process are found to experience degradation under white light aging and EL aging with the degradation rate of fluorescein less than that of DCJTB. The luminous efficacy of bar-coated warm white hybrid EL films is only 0.5 $\mathrm{lm} / \mathrm{W}$, which is less understood and needs detailed understanding. 


\section{Chapter 5}

\section{MODELING OF FREQUENCY-DEPENDENT COLOR SHIFT}

\subsection{Introduction}

In Chapter 3 and 4, the luminous efficacy values of warm white hybrid EL films produced by wet stamping and bar coating processes are only 1.45 and $0.5 \mathrm{~lm} / \mathrm{W}$ respectively. This low luminous efficacy is partly due to the luminous loss during the downshifting process in the organic dye layers. However, a larger part lies in the ACPEL devices with the luminous efficacy of reversed green of only $3.65 \mathrm{~lm} / \mathrm{W}$. A few research works have been attempted to enhance the luminous efficacy. Kim et al. inserted shortened single wall carbon nanotube (SWCNT) between dielectric and metal layers or between dielectric and phosphor layers [161]. A $65 \%$ increase in luminous efficacy was reported with the maximum efficacy at only about $3.50 \mathrm{~lm} / \mathrm{W}$. Wang et al. deposited a $\mathrm{SiN}_{\mathrm{x}}$ and $\mathrm{SiO}_{\mathrm{x}}$ thin film between electrode and dielectric layer as a electret layer [162]. The modified ACPEL devices exhibited a highest luminous efficacy at $2.3 \mathrm{~lm} / \mathrm{W}$. In another study, the luminous efficacy was increased from $3.14 \mathrm{~lm} / \mathrm{W}$ to $12.56 \mathrm{~lm} / \mathrm{W}$ by adding $\mathrm{TiO}_{2}$ nanoparticles to $\mathrm{BaTiO}_{3}$ dielectric layer [163]. A recent work by Wang and Chen [164] incorporated SWCNT and multiwall carbon nanotube (MWCNT) into $\mathrm{BaTiO}_{3}$ dielectric layer but only obtained a maximum efficacy of less than $1.4 \mathrm{~lm} / \mathrm{W}$. Although utilizing nanoparticles in ACPEL devices improved the luminous efficacy, its performance is still incomparable to LED. Therefore, the cause of this low luminous efficacy is necessary to be investigated.

As observed experimentally, the colors of EL films can be tuned by changing the operating frequency. An increase of frequency causes a blue shift of the ACPEL devices. This phenomenon is observed in both forward and reversed blue/green devices. In this chapter, a detailed study of this frequency effect on device colors is carried out. The excitation 
mechanism, luminescent centers and recombination mechanism of $\mathrm{ZnS}$ phosphor powders are reviewed. Several existing models for frequency dependent spectral composition are found to contradict with some of the experimental findings. A charge transfer and relaxation model is proposed, from which the low luminous efficacy is understood from $\mathrm{ZnS}$ level.

\subsection{Experimental methods}

Four types of samples were fabricated using a screen printing process: forward blue, reversed blue, forward green and reversed green. The printing processes for forward and reversed EL films are the same as those in Chapter 3. The structures of forward and reversed EL films are illustrated in the insets of Figures 3.1 and 3.2. The EL spectra and luminance of these samples were acquired using spectraradiometer PR655 from Photo Research. The measurement was performed at a $2^{\circ}$ observer angle with a spot size of about 5 $\mathrm{mm}$ in a dark enclosure at ambient conditions. The $\mathrm{AC}$ sinusoidal excitation voltages were applied by a Pacific 105-AMX power source without any DC bias voltage. The frequency effects were studied by supplying the voltage of $110 \mathrm{~V}$ with various frequencies of $100 \mathrm{~Hz}$, $400 \mathrm{~Hz}, 800 \mathrm{~Hz}, 1200 \mathrm{~Hz}$, and $1600 \mathrm{~Hz}$. Voltages of $80 \mathrm{~V}, 110 \mathrm{~V}, 140 \mathrm{~V}, 170 \mathrm{~V}$ and 200 V were supplied with a fixed frequency at $400 \mathrm{~Hz}$ to study the voltage effect on the device color. The optical properties were processed and calculated by the SpectraWin software. The multipeak fitting of EL spectra was achieved using Origin software.

\subsection{Experimental results}

\subsubsection{Voltage effects}

The EL spectra of forward blue, reversed blue, forward green and reversed green operated at $400 \mathrm{~Hz}$ and different voltages are shown in Figure 5.1. The increase of voltage from 80 $\mathrm{V}$ to $200 \mathrm{~V}$ consistently increases the EL spectra intensity levels for all four devices. The 


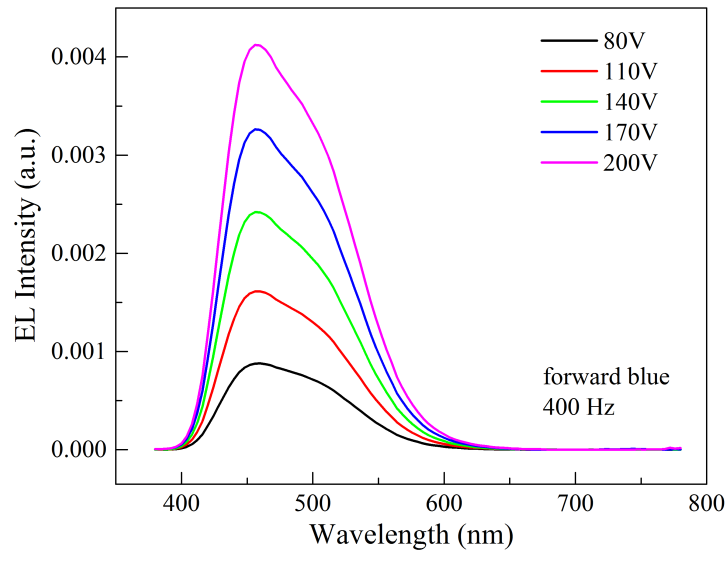

(a)

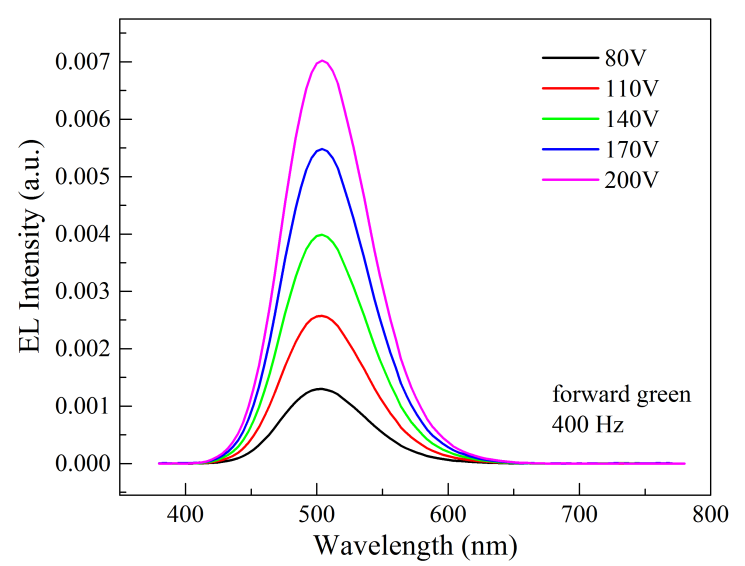

(c)

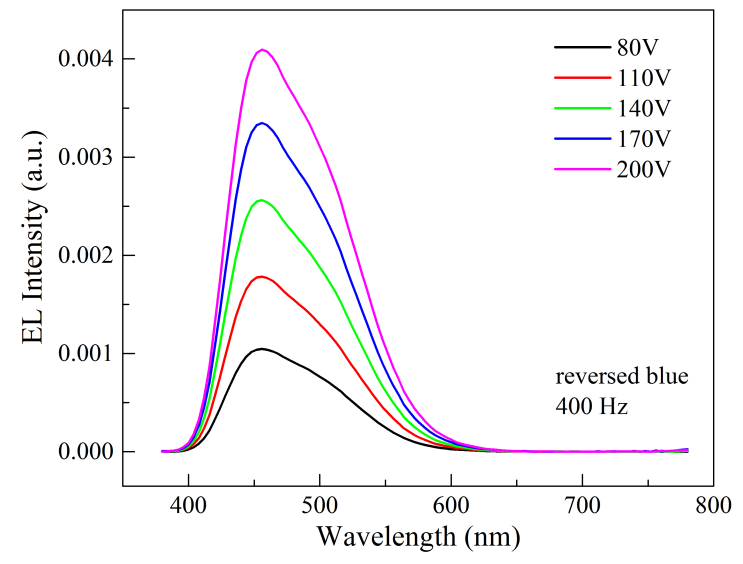

(b)

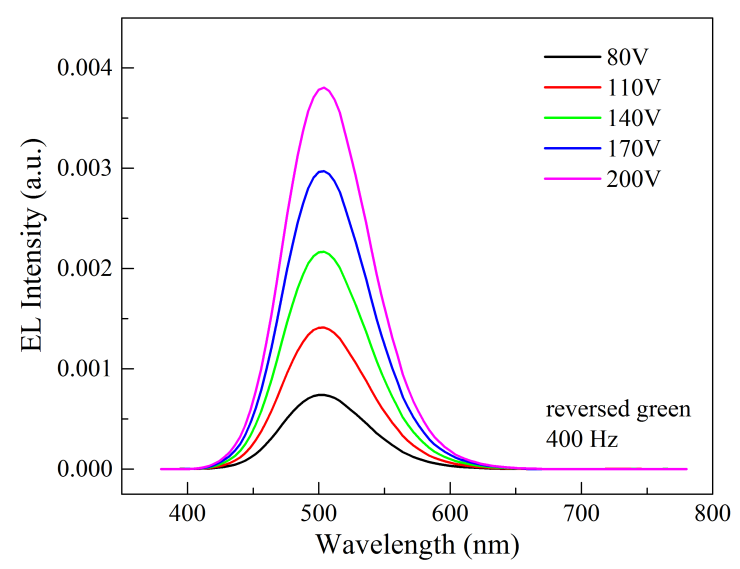

(d)

Figure 5.1: EL spectra of (a) forward blue, (b) reversed blue, (c) forward green, and (d) reversed green operated at $400 \mathrm{~Hz}$ and different voltages.

normalized spectra shown in Figure 5.2 show negligible changes of emission bands for the four devices. Therefore, it is concluded that the change of operating voltage or the electric field over the ACPEL devices does not affect the emission shapes or colors.

\subsubsection{Frequency effects}

When EL films are operated at different frequencies, the EL intensities increase in all wavelengths for forward blue, reversed blue, forward green and reversed green devices as shown in Figure 5.3. This is due to the increased number of recombination cycles per unit time with the increase of frequency. Therefore, the luminance also increases with frequency as 


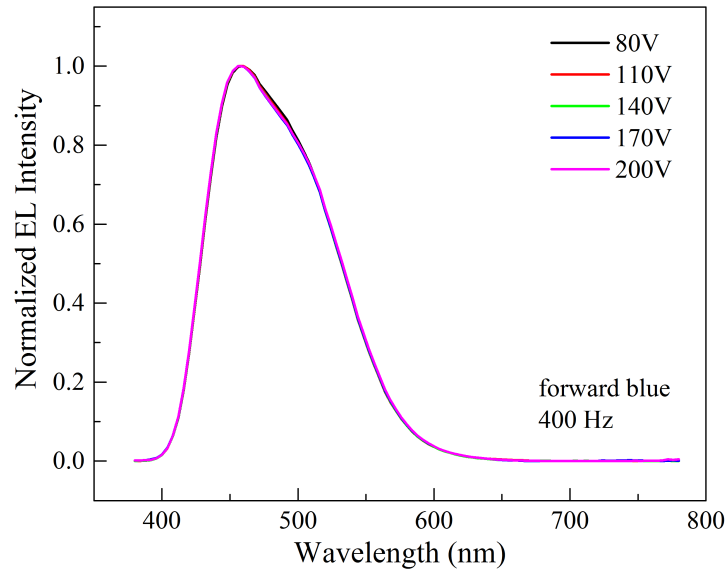

(a)

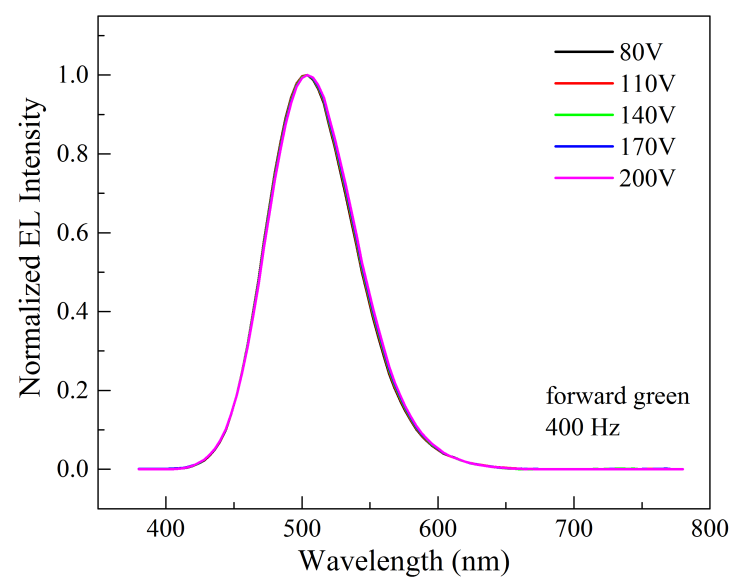

(c)

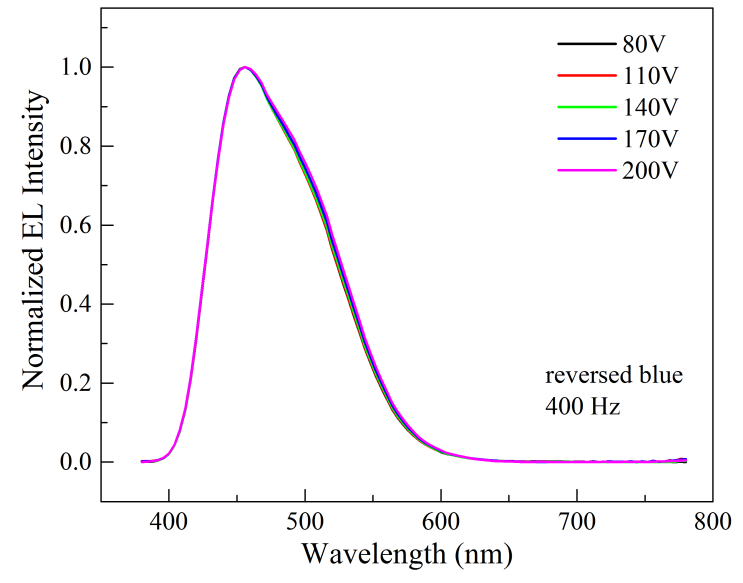

(b)

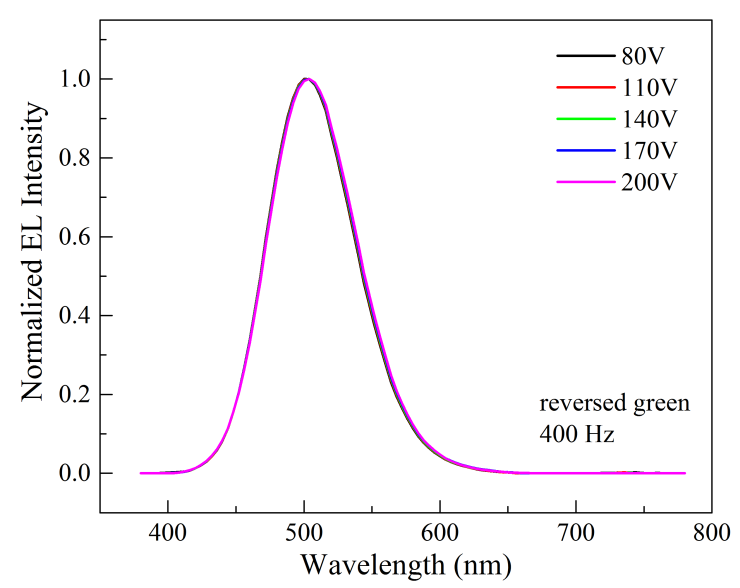

(d)

Figure 5.2: Normalized EL spectra of (a) forward blue, (b) reversed blue, (c) forward green, and (d) reversed green operated at $400 \mathrm{~Hz}$ and different voltages.

shown in Figure 5.4(a). However, the luminance per cycle as indicated in Figure 5.4(b) decreases with frequency for all four devices and tends to reach a steady state at higher frequencies. The EL spectrum shapes for forward and reversed green in Figures 5.3(c) and 5.3(d) do not exhibit noticeable change with the increase of frequency. However, the forward and reversed blue devices have, interestingly, dominant green emissions at $100 \mathrm{~Hz}$. The Shift of EL spectra is more obvious under the normalized EL spectra in Figure 5.5. A clear dominant green emission is noticed in both forward blue and reversed blue when operated at $100 \mathrm{~Hz}$. For all the four devices, the peak frequency experiences a blue shift with 


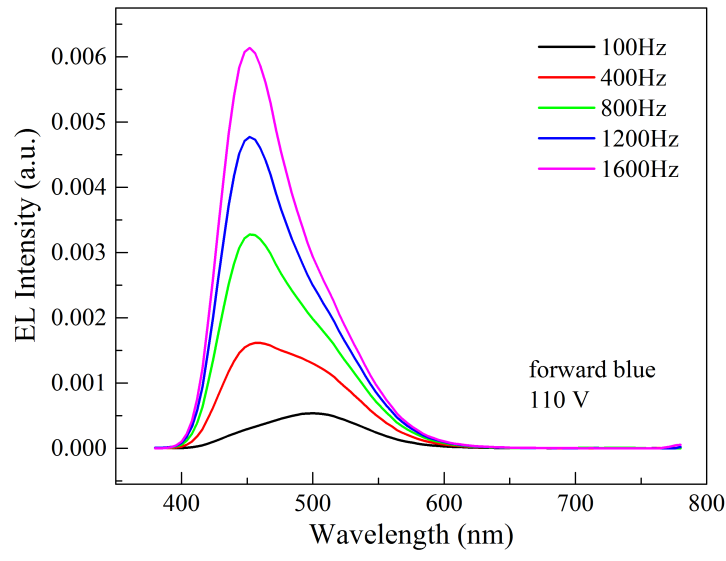

(a)

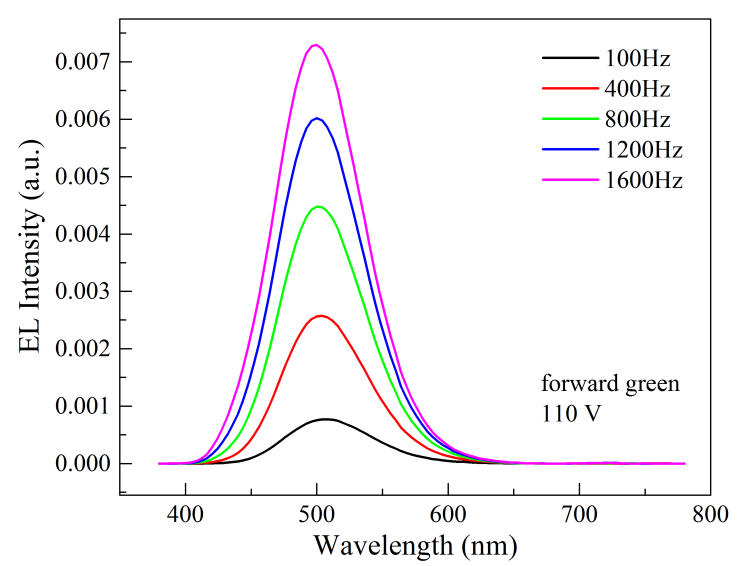

(c)

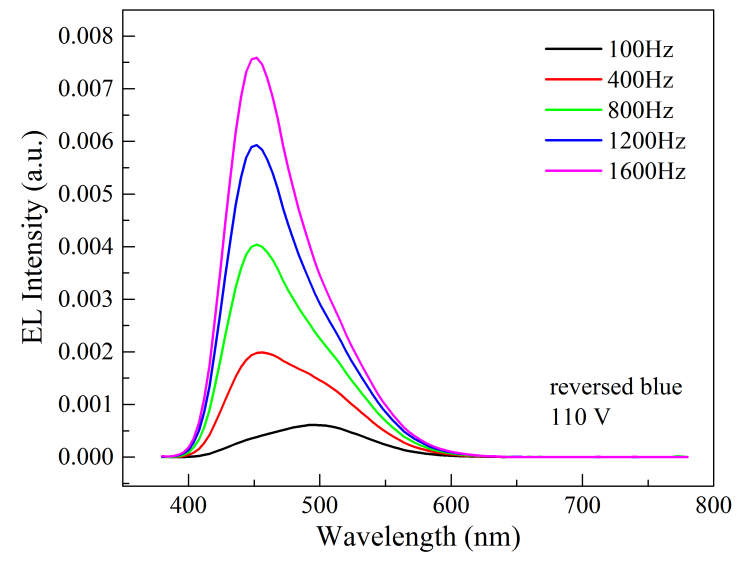

(b)

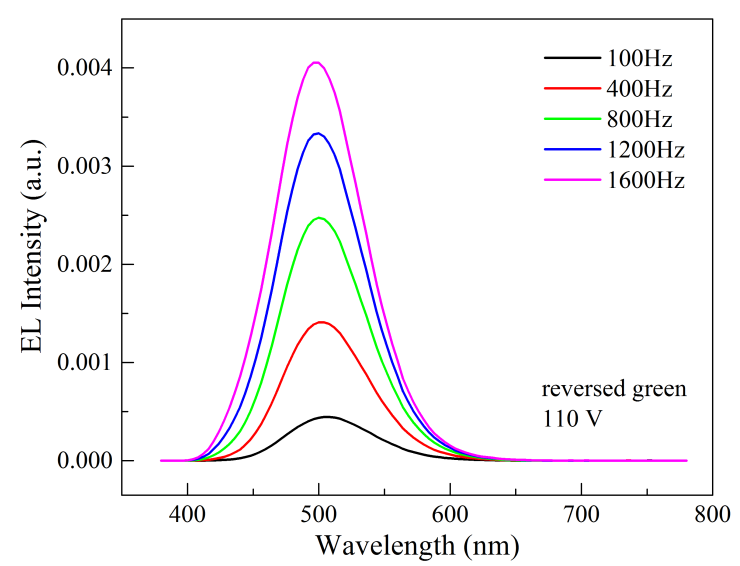

(d)

Figure 5.3: EL spectra of (a) forward blue, (b) reversed blue, (c) forward green, and (d) reversed green operated at $110 \mathrm{~V}$ and different frequencies.

the increase of frequency, among which the shift in green devices is less profound. With the similar behaviors between forward and reversed devices, it can be concluded that the frequency dependent color shift is not caused by the charge polarization at the electrodes, which is different from Sadakata et al.'s work on ITO/Tetracene/Al diodes [165].

As suggested by earlier works $[92,166]$, the EL spectrum of $\mathrm{ZnS}$ phosphors can be decomposed to four emission lines. Ibaez et al. decomposed the EL spectrum of $\mathrm{ZnS}$ phosphors operated at $3 \mathrm{kHz}$ into emission lines with the peak wavelengths of 448, 471, 504, and $550 \mathrm{~nm}$, each following a Gaussian distribution [166]. In Stanley's work, the emission spectra of $\mathrm{ZnS}: \mathrm{Cu}, \mathrm{Cl}$ particles were simulated by four emission lines with the 


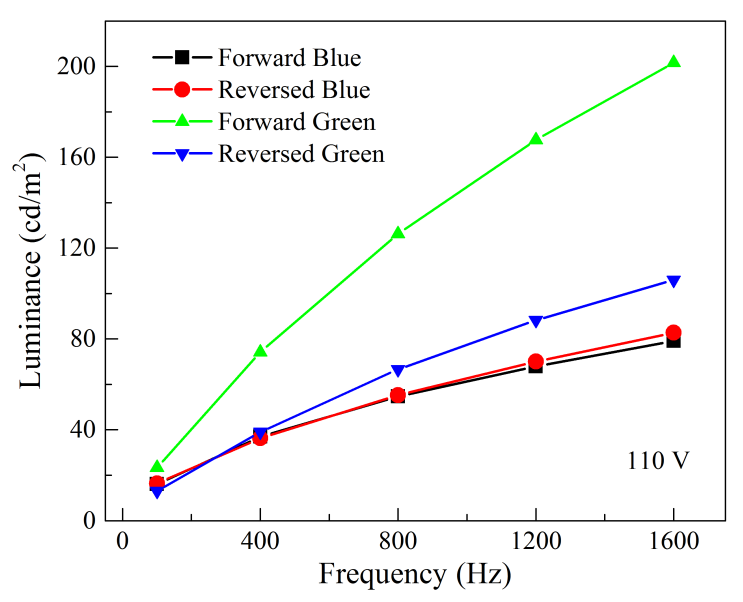

(a)

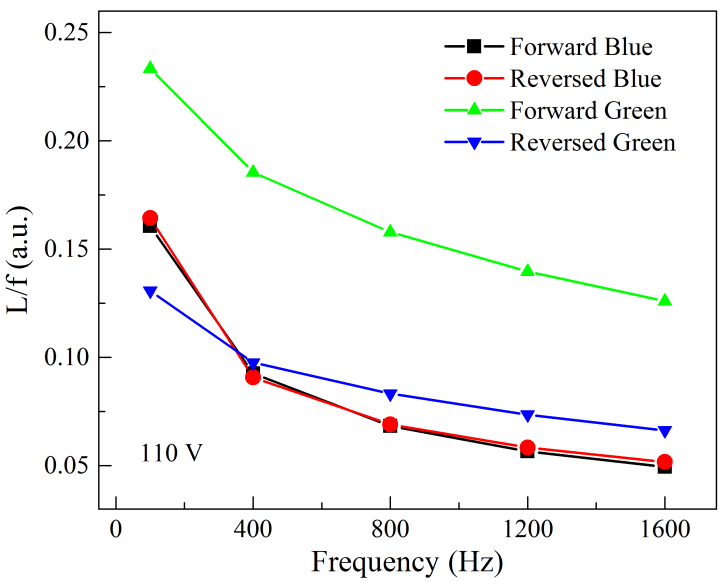

(b)

Figure 5.4: (a) Luminance and (b) luminance per cycle as a function of frequency of forward blue, reversed blue, forward green, and reversed green EL films operated at $110 \mathrm{~V}$.

Gaussian peaks of 452, 464, 475, and $508 \mathrm{~nm}$ [92]. The exact composition of emission lines depends largely on the production process and dopant concentrations. In our case, the EL spectrum of blue $\mathrm{ZnS}$ phosphors are best described by three emission lines with the peak wavelengths of 444, 470, and $506 \mathrm{~nm}$. Each emission line follows a Gaussian distribution:

$$
y=y_{0}+A e^{-\frac{\left(x-x_{c}\right)^{2}}{2 w^{2}}}
$$

where $x_{c}$ is the peak wavelength, $w$ the width and $A$ the peak intensity. For the three emission lines of the blue $\mathrm{ZnS}$ phosphors, the simulated Gaussian distributions have common $x_{c}$ and $w . w$ values are kept at $16.5 \mathrm{~nm}, 23.4 \mathrm{~nm}$, and $36.2 \mathrm{~nm}$ for the peak wavelengths of 444, 470 and $506 \mathrm{~nm}$ respectively. Only the peak intensity $A$ varies at different frequencies. The same methodology is applied for the decomposition of the EL spectra of green $\mathrm{ZnS}$ phosphors at different frequencies. The peak wavelengths of the three emission lines are 470,506 and $560 \mathrm{~nm}$ with fixed widths of 16.9, 27.0, and $23.3 \mathrm{~nm}$ respectively. As shown in Figure 5.7, the cumulative fittings show satisfactory goodness of fittings with the $R^{2}$ values of $0.99955,0.99916,0.99892$, and 0.99905 for forward blue, reversed blue, forward green, and reversed green devices respectively. 


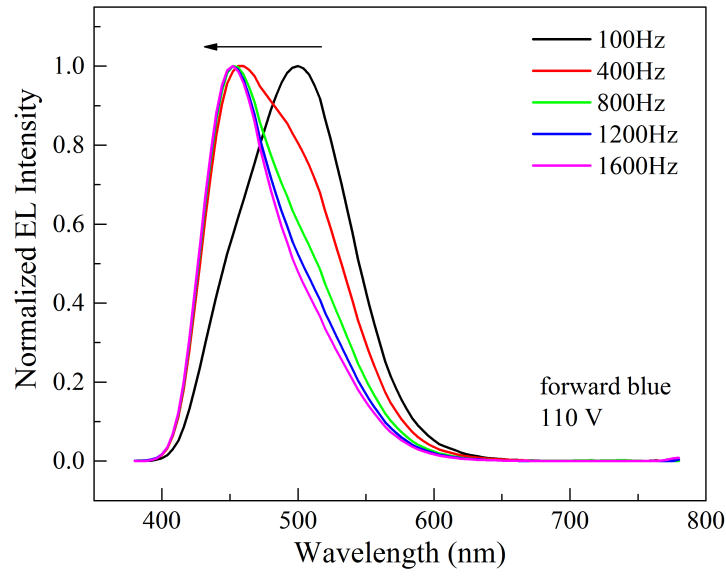

(a)

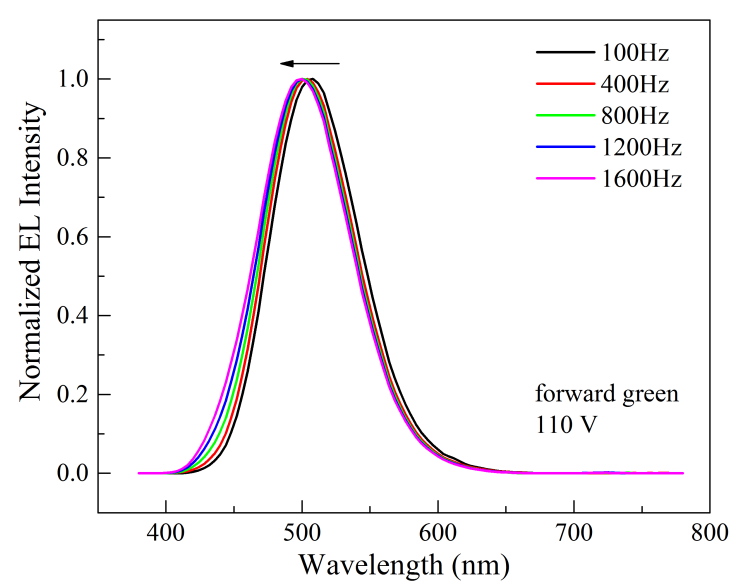

(c)

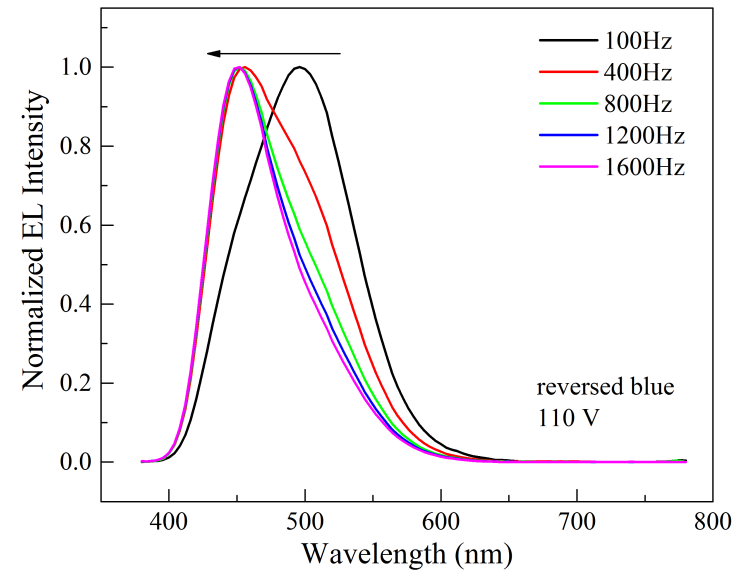

(b)

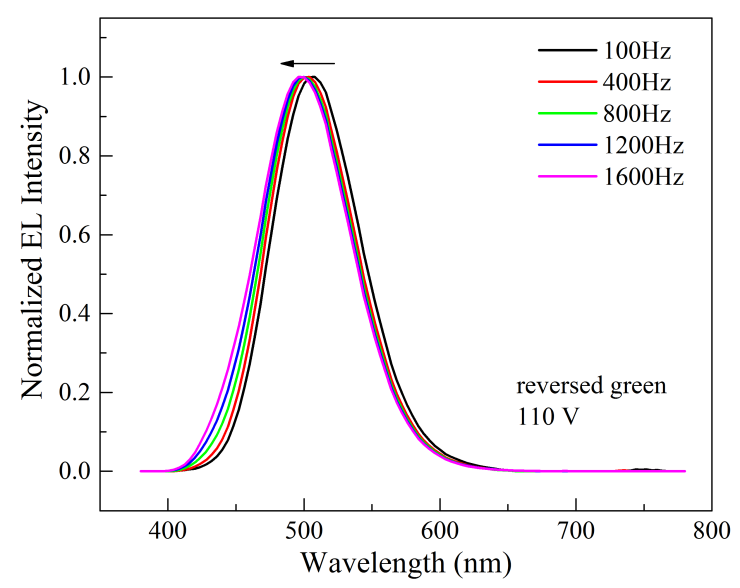

(d)

Figure 5.5: Normalized EL spectra of (a) forward blue, (b) reversed blue, (c) forward green, and $(d)$ reversed green operated at $110 \mathrm{~V}$ and different frequencies.

The fitted peak intensities for each emission lines of blue and green $\mathrm{ZnS}$ phosphors are further divided by the frequency and are plotted against the operating frequency as shown in Figure 5.7. In forward and reversed blue devices as shown in Figures 5.7(a) and 5.7(b), the $506 \mathrm{~nm}$ peak intensity per cycle decreases with frequency and tends to reach steady state at high frequencies while the 444 and $470 \mathrm{~nm}$ peak intensity per cycle increases with frequency and seems to reach equilibrium from $800 \mathrm{~Hz}$ but with a small downward trend. Similarly for forward and reversed green devices, the 506 and $560 \mathrm{~nm}$ peak intensity per cycle decreases with frequency. Although the steady state level is not reached, the decre- 


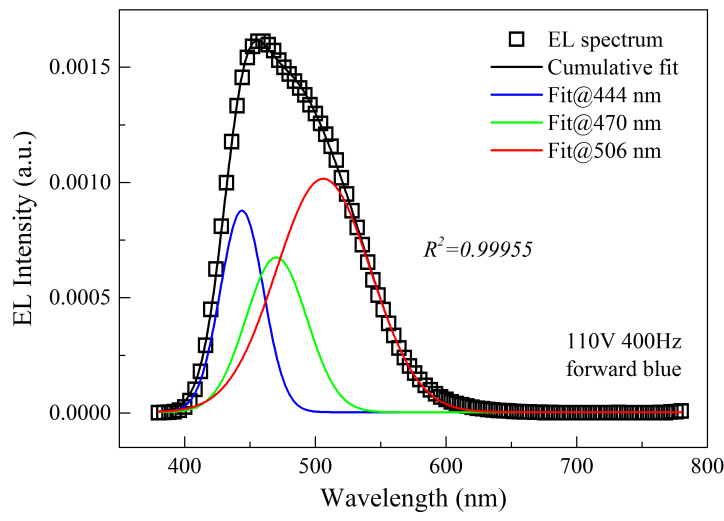

(a)

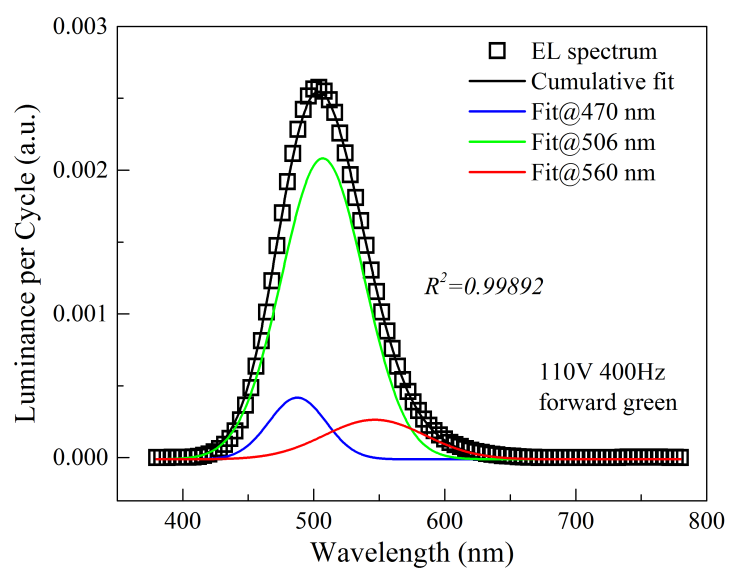

(c)

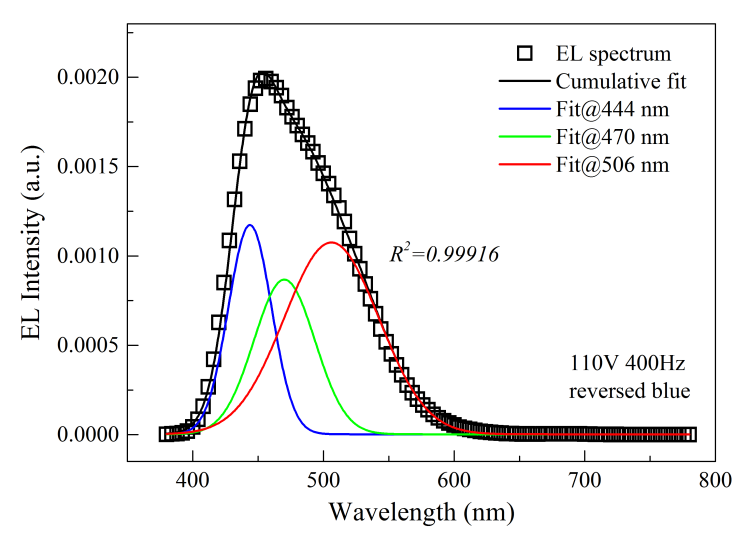

(b)

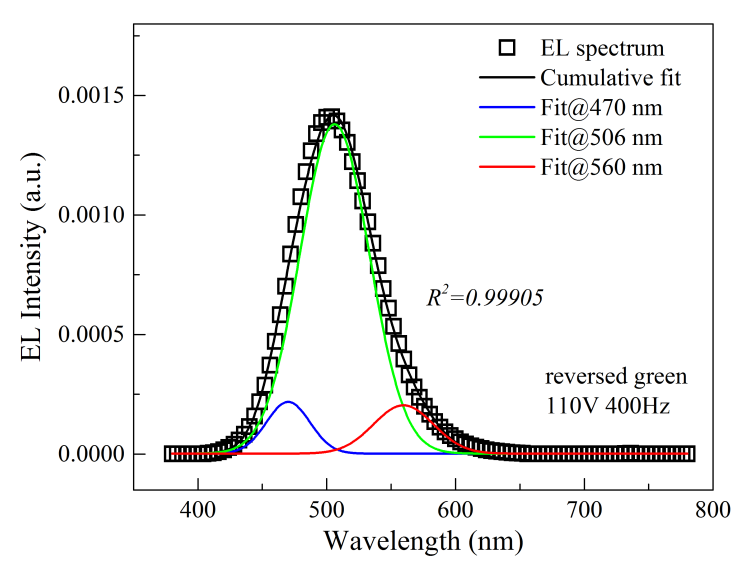

(d)

Figure 5.6: The decomposition of EL spectra operated at $110 \mathrm{~V}$ and $400 \mathrm{~Hz}$ into three emission lines for (a) forward blue, (b) reversed blue, (c) forward green, and (d) reversed green.

ment rate is reduced with frequency. The $470 \mathrm{~nm}$ peak intensity meanwhile increases with frequency and the increment rate gradually slows down. The forward and reversed devices for both green and blue phosphors behaves in a similar fashion. This again eliminates the possibility of the electrode relaxation process affecting the frequency dependent color shifting behaviors. 


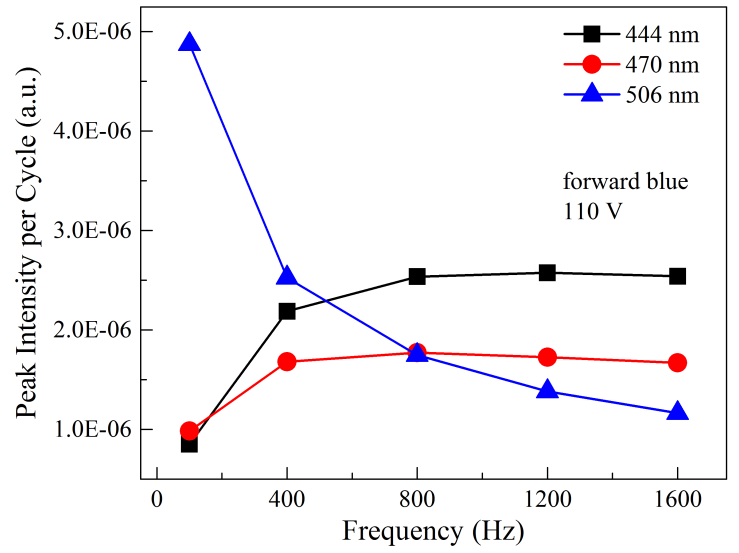

(a)

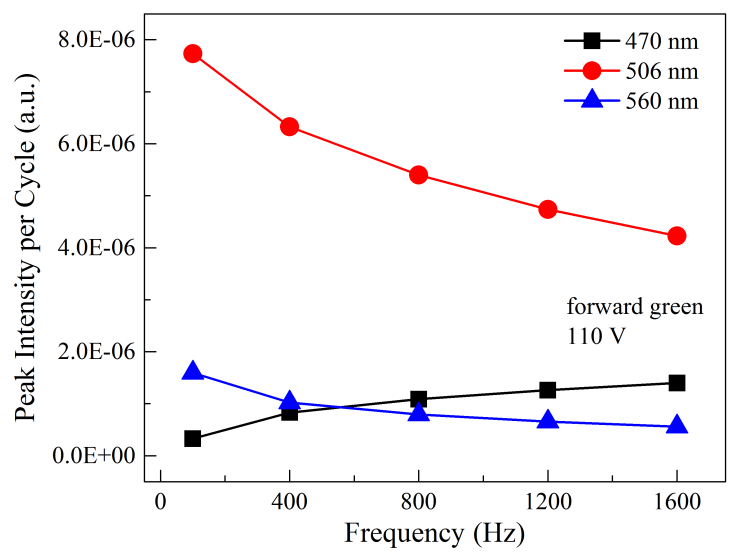

(c)

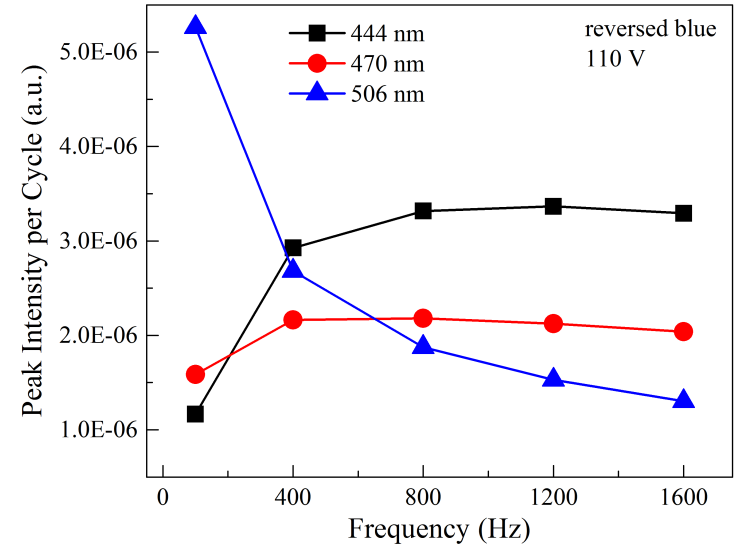

(b)

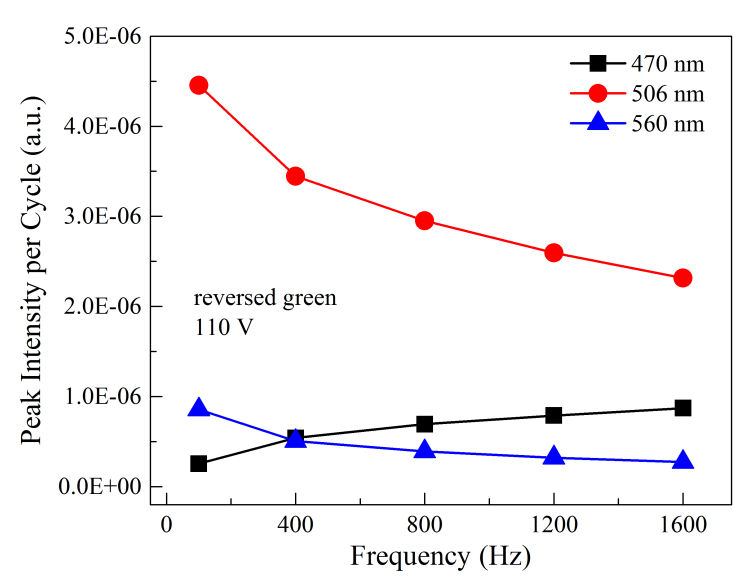

(d)

Figure 5.7: The peak luminance intensities of decomposed emission lines per cycle as a function of frequency for (a) forward blue, (b) reversed blue, (c) forward green, and (d) reversed green.

\subsection{Previous models}

The two dominant light emission theories of ACPEL devices have been reviewed in Section 2.1.5. The Fischer's bipolar field emission model assumes the presence of tiny $\mathrm{Cu}_{2} \mathrm{~S}$ needles, around which electric field is intensified and electrons and holes are field-emitted [46]. The frequency dependent color shifting is proposed by Fischer to be due to the redistribution of charges at various frequencies as shown in Figure 5.8. At frequencies as low as $10 \mathrm{~Hz}$, charges spread into bulk $\mathrm{ZnS}$ where green $\mathrm{Cu}$ emission centers are dominant. At 


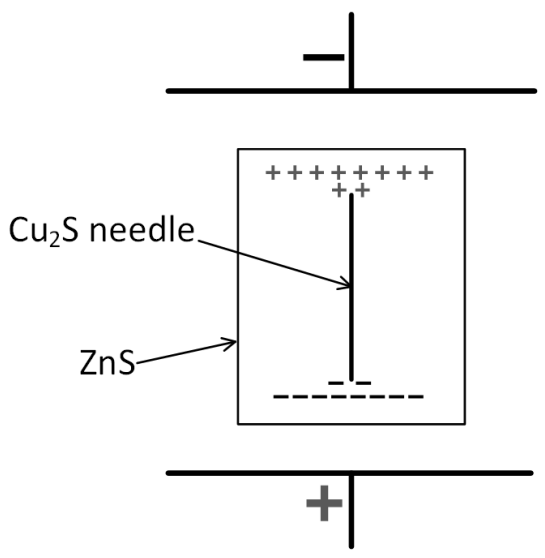

$10 \mathrm{~Hz}$

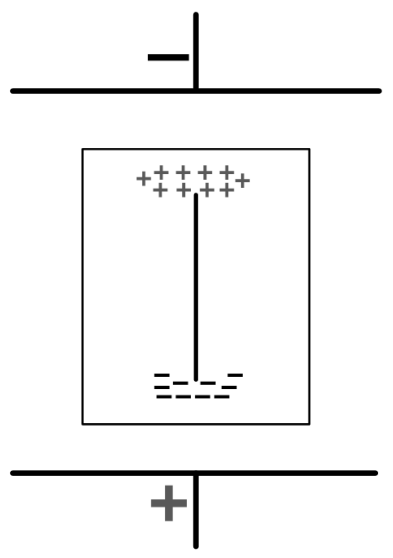

$500 \mathrm{~Hz}$

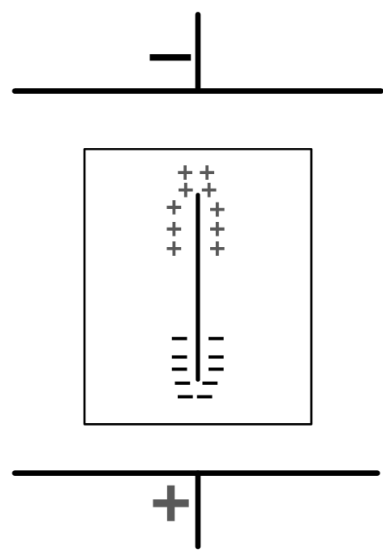

$10 \mathrm{kHz}$

Figure 5.8: Redistribution of injected charge due to drift in an applied field in dependence of frequency according to Fischer's bipolar field emission model.

frequencies up to $10 \mathrm{kHz}$, charges are concentrated near the $\mathrm{Cu}_{2} \mathrm{~S}$ needles where blue $\mathrm{Cu}$ emission centers are dominant. However, no mathematical model or detailed mechanism of the charge distribution along with frequency is proposed.

Another junction model proposed by Brovetto et al. is based on the pn junction along defect sites, where electron-hole pairs are produced due to the tunneling of electrons from the valence to conduction band [81]. The treatment of $\mathrm{ZnS}$ blue and green luminescent centers is based on the Prener and Williams's model [167]. As shown in Figure 5.9, the center responsible for green emission is constituted by a pair of acceptor and donorlike ions separated by a distance $r$. Blue emission is proposed to be due to the transition of electrons from conduction band to blue emission centers. The probability $\left(\beta_{\mathrm{g}}\right)$ for green emission transition is governed by the following equation:

$$
\beta_{g}(r)=\beta_{g, \max } \exp \left[-r / R_{D}\right]
$$

where $R_{D}$ is the half Bohr radius of the shallower hydrogenic state and $\beta_{g, \max }$ the maximum transition probability. The exciton generation rate $\sigma(\nu)$ is defined in this case to be 
proportional with the operating frequency $\nu$.

$$
\sigma(\nu)=\frac{q_{r}}{e \Omega} 2 \nu
$$

where $q_{r}$ is the charge injected during each half cycle through the pn junction, $\Omega$ the volume of the lattice regions where recombination take place. $q_{r}$ was found to follow the following relationship with frequency:

$$
q_{r}=\frac{1}{\pi}\left(\frac{\sqrt{V}}{c}\right)^{\frac{1}{2}} I_{0} \exp \left[-\frac{c}{\sqrt{V}}\right] \nu^{-1}-\frac{1}{4 \pi^{2} V_{l} 0} I_{0}^{2} \exp \left[-\frac{2 c}{\sqrt{V}}\right] \nu^{-2}+O\left(\nu^{-3}\right)
$$

where $V$ is the applied voltage amplitude, $I_{0}$ the initial current, $V_{l 0}$ the voltage drop during charge accumulation, and $c$ a constant. In the junction model treatment, at high enough frequencies all the green bands are filled and the EL emission takes place only within the blue band. As the frequency decreases, the electron and hole distributions are not at the equilibrium levels due to the slower pair production. Through a complicated analytical derivation, the brightness ratio of green and blue emissions $B_{g} / B_{b}$ becomes

$$
\frac{B_{g}}{B_{b}}=\frac{\beta_{g}}{\beta_{b}} \frac{n_{g}}{n_{c}} \frac{m_{g}}{m_{b}}
$$

where $\beta_{g}$ and $\beta_{b}$ are the recombination coefficients of green and blue emissions, $n_{g}$ the electron concentration bound to green emission centers, $n_{c}$ the electron concentration in the conduction band, $m_{g}$ and $m_{b}$ concentration of holes bound to green and blue emission centers.

$$
\begin{gathered}
\frac{n_{g}}{n_{c}}=\frac{\alpha_{e g} N_{g}^{2}}{\sigma \nu+N_{g} s_{e} \exp \left[-E_{D} / k T\right]} \\
\frac{m_{g}}{m_{b}}=\frac{N_{g}}{N_{b}} \frac{\sigma(\nu)+\left[N_{b}+\left(\alpha_{h g} / \alpha_{h b}\right) N_{g}\right] s_{h b} \exp \left[-E_{A b} / k T\right]}{\sigma(\nu)+\left[N_{g}+\left(\alpha_{h b} / \alpha_{h g}\right) N_{b}\right] s_{h g} \exp \left[-E_{A g} / k T\right]}
\end{gathered}
$$

where $N_{g}$ and $N_{b}$ are the concentrations of the green and blue emission centers, $\alpha_{e g}$ the 


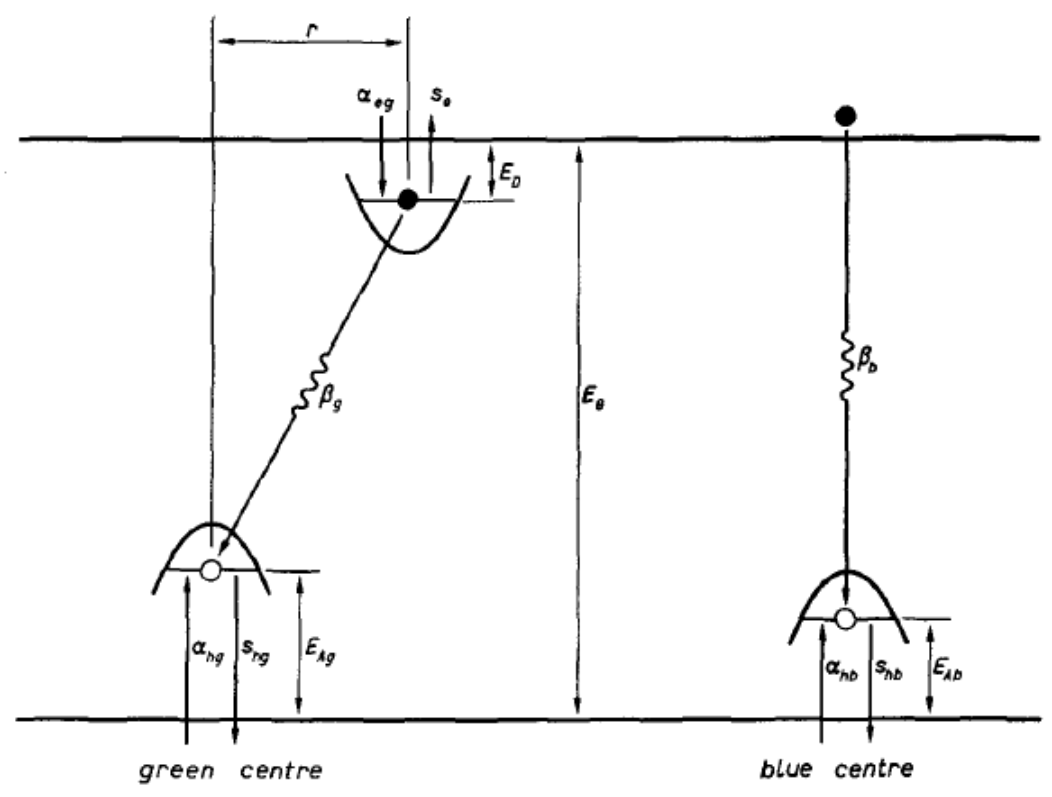

Figure 5.9: Band diagram for the blue and green emission centers in $\mathrm{ZnS}: \mathrm{Cu}$ phosphors.

probability factor of electrons trapped in green centers, $\alpha_{h g}$ and $\alpha_{h b}$ the probability factors of holes trapped in green and blue centers, $s_{e}, s_{h g}$ and $s_{h b}$ frequency factors for thermal ionization of electrons and holes in various centers. $\beta_{g}$ and $\beta_{g}$ are found to be only dependent on temperature and are not affected by frequency. This model contributes the color shift mainly to the frequency-dependent charge injection and space charge build-up. This is hard to justify without the comparison of the simulated results with experimental results. Moreover, theoretically, the time scale for charge injection is very small while the space charge build-up may be within the range of time scales as operating frequencies.

\subsection{Proposed model}

From the above experimental results and previous models trying to elucidate the frequency dependent color shift mechanisms, a charge transport and relaxation model is proposed in our study to include the recent findings on the excitation mechanism, luminescent centers and recombination mechanisms. The analytical expression of the proposed model will be derived and compared with experimental results. 


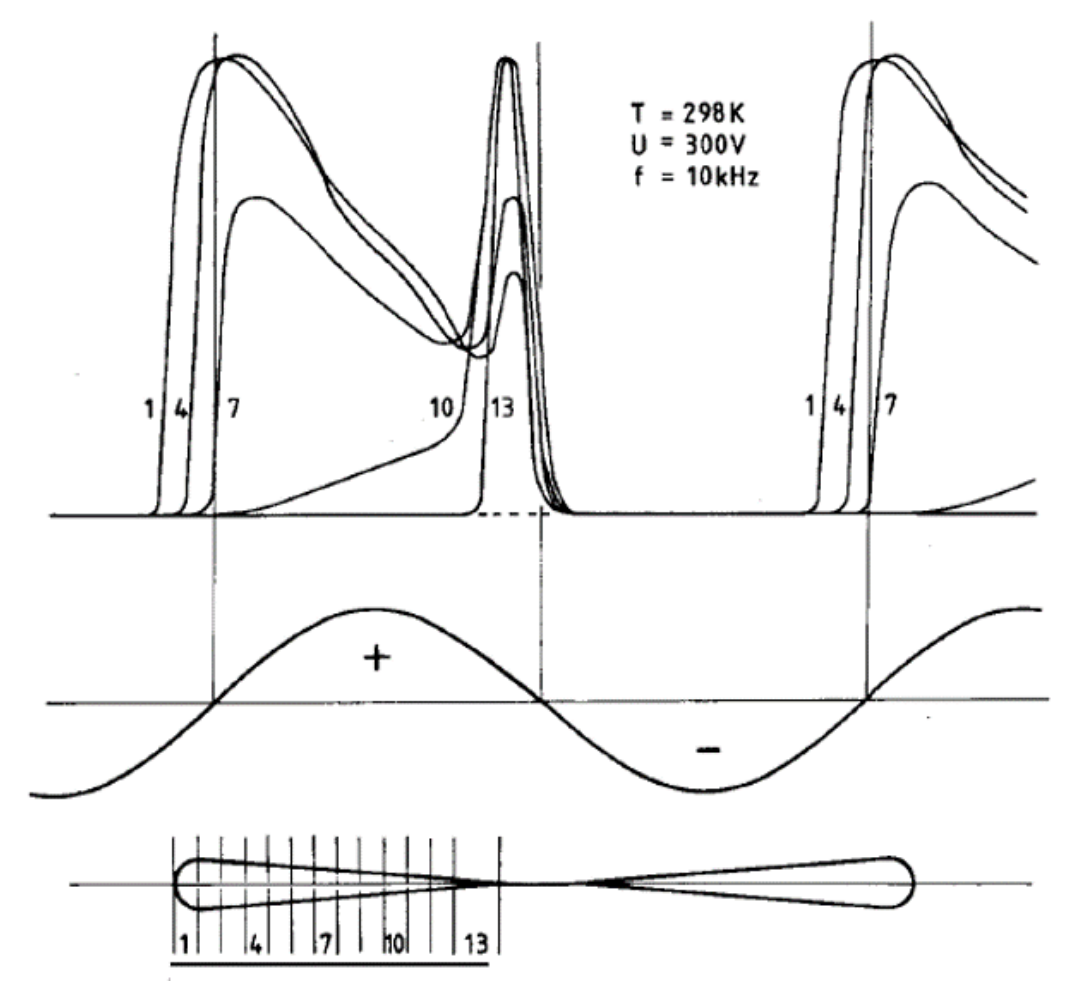

Figure 5.10: Brightness waves of different comet segments at sine wave excitation. Each curve represents the brightness wave of a thirteenth of the comet. The parameter refers to the measured part of the comet: 1, comet head, 212 , the subsequent parts, 13 , comet tail (see lower part of the figure). The curves are slightly smoothed copies of the originally recorded curves, the amplitude being normalized to the maximum of each brightness wave [168].

\subsubsection{Excitation mechanism}

In the 1960s, Fischer's model was proposed mainly based on the observation of the doublecomet needles [45, 46]. Electrons/holes are filed-emitted from one comet at each half cycle. At the next half cycle, emitted electrons will recombine with the trapped holes from the last half cycle. Therefore, bipolar field reversal is necessary to the light emission mechanism. However, a unipolar AC field also produces similar emission waveforms [97]. Moreover, the comet tail sees a light emission during the voltage fall at each half cycle of the sinusoidal excitation signal [168], as shown in Figure 5.10. If Fischer's model stands, the comet tail should have a minimal light output. Even with the assumption that there are tiny small needles at the comet tail, the length of the tiny needle is very small. According to Equation 


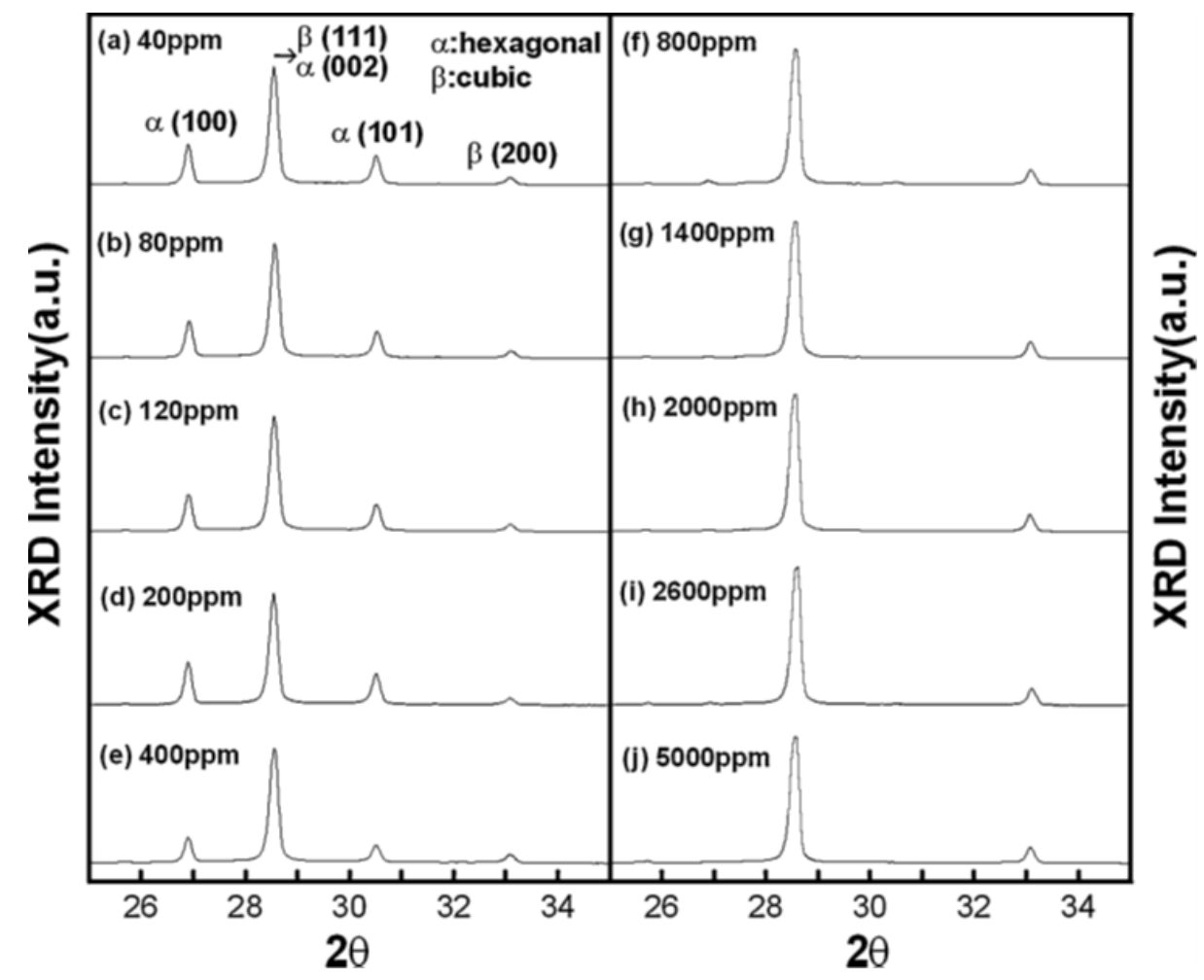

Figure 5.11: $\mathrm{XRD}$ spectra of $\mathrm{ZnS}: \mathrm{Cu}, \mathrm{Cl}$ with $\mathrm{Cu}$ addition in the range of 40 to 5000ppm fired at $900{ }^{\circ} \mathrm{C}[78]$.

2.1, the field-emitted charges are limited in quantity and thus the light output is still small at the comet tail. Therefore, the bipolar field emission of Fischer's model may not be able to explain these two observations.

Later, the junction model presumes the presence of pn junction at the defect sites [81]. However, the p-type semiconductor is proven not feasible in $\mathrm{ZnS}$ bulk materials. A valuable takeaway from this model is the possibility of exciton initiation at the $\mathrm{Cu}$-doped defect sites. A recent study over the electroluminescence of $\mathrm{Cu}$-doped $\mathrm{ZnS}$ powders shows that there is a phase shift from hexagonal to cubic with the increase of $\mathrm{Cu}$ concentration [78]. As shown in Figure 5.11, both cubic and hexagonal phases coexist within the $\mathrm{ZnS}: \mathrm{Cu}, \mathrm{Cl}$ particles up to 400 ppm. From 800 ppm onwards, the hexagonal phase disappears and electroluminescence emerges. Therefore, the powder electroluminescence of $\mathrm{ZnS}$ phosphors is associated with the $\mathrm{Cu}$-assisted phase transition.

TEM analysis of the cross section of $\mathrm{ZnS}$ powders reveals a narrow intergrowth struc- 
ture as shown in Figure 2.8(b) [80]. This structure is a twin grain boundary defect along [111] direction. A SEM image of the hydrochloric acid etched $\mathrm{ZnS}: \mathrm{Cu}$ particles in Fig. 2.8(a) shows a layered structure along the [111] direction and pyramids at one end [79]. Another quantitative element analysis of $\mathrm{ZnS}: \mathrm{Cu}$ crystals indicates that the twin boundary areas are rich in $\mathrm{Cu}$ [169]. $\mathrm{ZnS}$ powders are produced by quenching from the wurtzite phase above $1030{ }^{\circ} \mathrm{C}$, which leaves localized wurtzite phase embedded within cubic structure [34]. The added $\mathrm{Cu}$ dopants act as nuclei for the promotion of a $2 \mathrm{H}-3 \mathrm{C}$ conversion, leading to the creation of twin boundaries along [111] twin axis.

In a further study of the cross section of etched $\mathrm{ZnS}$ phosphors in applied electric fields [55], the EL lines aligning with the electric field have the highest luminance while those perpendicular to the electric field are not electroluminescent. This suggests that the [111] twin boundary is the direct source of th EL excitation. This excitation may be initiated by field emission of electrons and holes from the surface traps at the twin boundaries. Assuming that the time it takes for the field emission is very fast, the current of emitted electrons is then dependent on the temperature $(T)$, the electric field near the twin boundary $(E)$, and the energy depth of surface traps.

\subsubsection{Luminescent centers and recombination mechanisms}

A light emission mechanism typically involves three steps: charge carrier generation, transfer and recombination. As discussed in the previous section, charges are generated at the [111] grain boundaries and need to travel to luminescent centers to enable light emission. Therefore, it is necessary to understand the nature and location of various luminescent centers in $\mathrm{ZnS}$ phosphor powders.

Many research works have been published to study the electroluminescence of $\mathrm{ZnS}$ phosphors doped with different activators and co-activators $[170,171]$. The luminescence with different concentrations of activators and coactivators is summarized in Figure 5.12. When only $\mathrm{Cu}$ is doped, only red emission is observed [172]. This luminescence is due to 


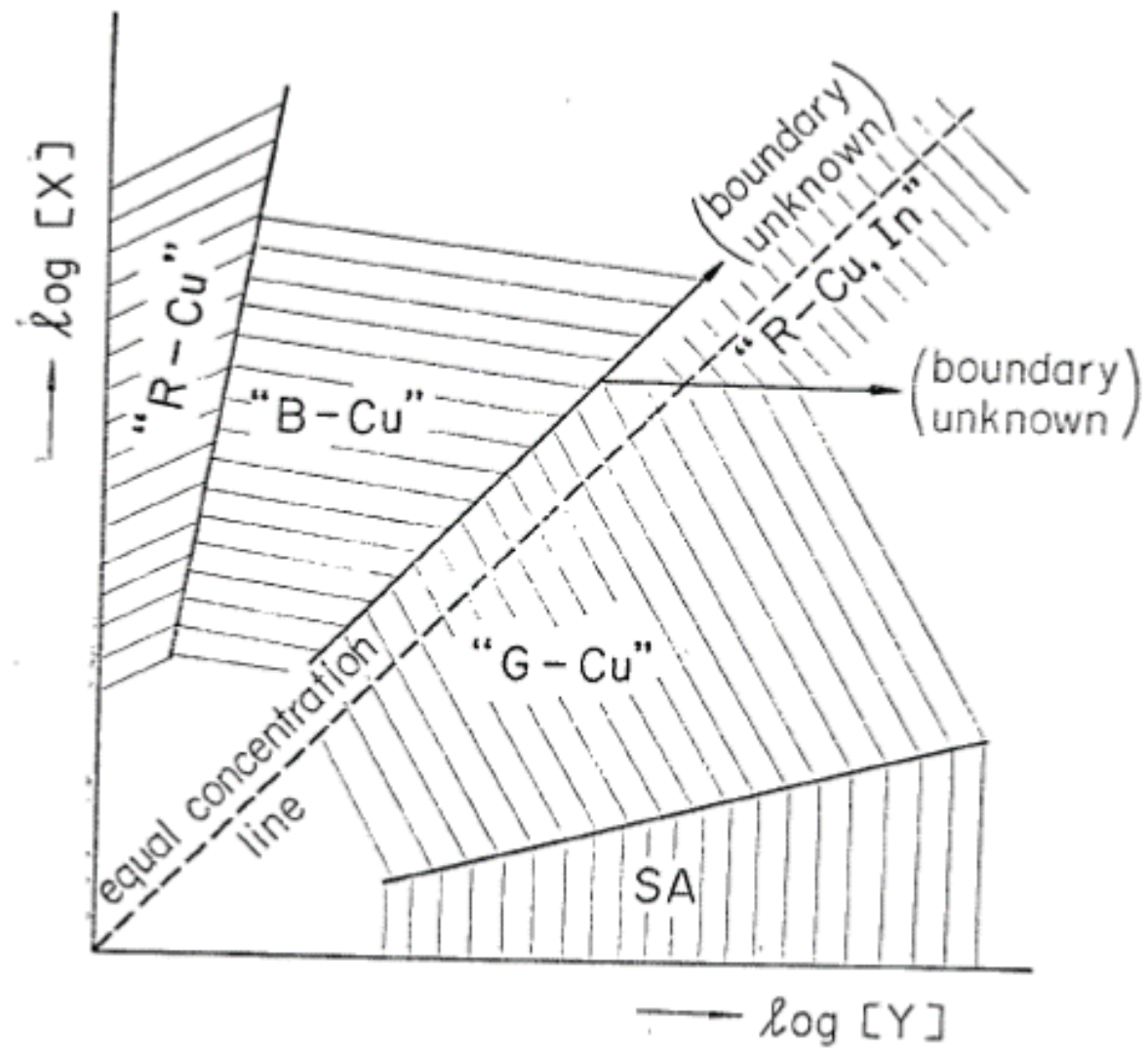

Figure 5.12: The relation between the kind of luminescence and the concentration of activator $(\mathrm{X})$ and coactivator $(\mathrm{Y}) . \mathrm{X}=\mathrm{Cu}, \mathrm{Ag}, \mathrm{Au}, \mathrm{Y}=\mathrm{Al}, \mathrm{Ga}$, In. "G-Cu": green luminescence in the case of $\mathrm{Cu}$; "B-Cu": blue luminescence in the case of $\mathrm{Cu}$; SA: self-activated luminescence (blue); "R-Cu": red luminescence in the case of $\mathrm{Cu}$; "R-Cu,In": red luminescence in the case of $\mathrm{Cu}$ and $\operatorname{In}(\mathrm{Ga})$ [77].

the transition from $\mathrm{S}$ vacancy to $\mathrm{Cu}$ luminescent centers [173]. No additional luminescence is detected without doping coactivators. In both Rothschild et al. and Kröger et al.'s study, no blue or green emissions are produced with the addition of $\mathrm{Cl}$ coactivators [170, 171]. With the equal amount of activator and coactivator, a green emission associated with $\mathrm{Cu}(\mathrm{G}-\mathrm{Cu})$ is produced. If the coactivator is higher in concentration than activator, the $\mathrm{ZnS}$ exhibits blue electroluminescence, whose luminescent center is named self-activator (SA). When the concentration of activator is higher than that of coactivator, blue EL is also produced and the associated luminescence center with $\mathrm{Cu}$ is named $\mathrm{B}-\mathrm{Cu}$. The detailed microstructure and composition of these three luminescence centers are discussed below. 


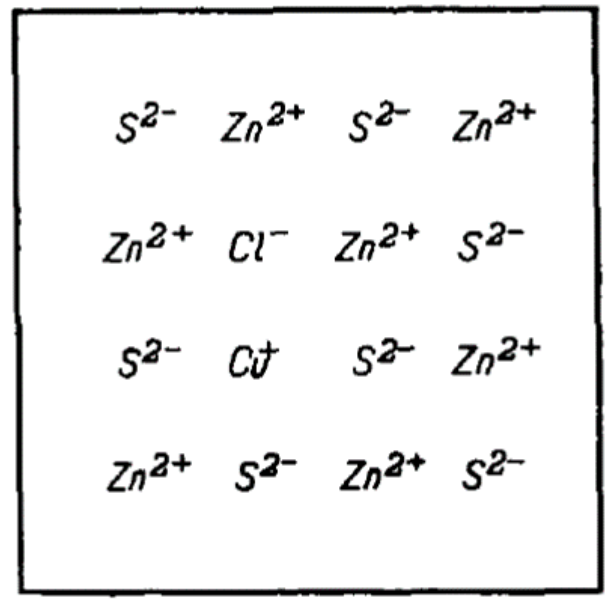

(a)

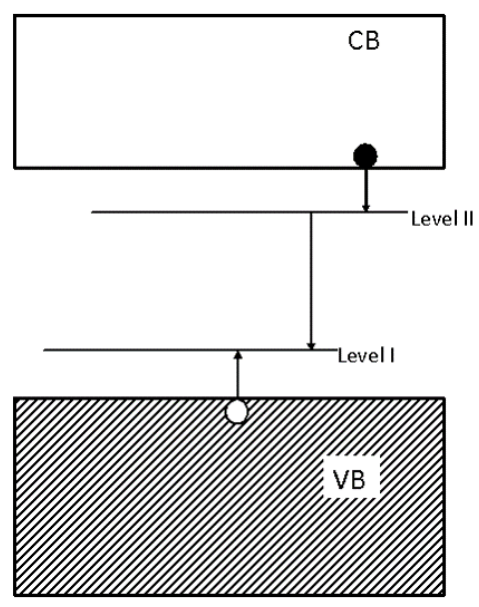

(b)

Figure 5.13: (a) Configuration in the zinc sulphide lattice caused by incorporation of $\mathrm{CuCl}$ green center [171]; (b) band diagram illustrating donor-acceptor recombination mechanism [174].

\section{G-Cu luminescent center}

Based on the susceptibility measurements by Bowers and Melamed [41], all ZnS samples are diamagnetic over a wide range of temperatures from 1.5-300 K. Moreover, Holton et al. do not find any ESR signals in unexcited $\mathrm{Cu}$-doped $\mathrm{ZnS}$ [175]. Therefore, copper is $\mathrm{Cu}^{+}$in all unexcited $\mathrm{Cu}$-doped $\mathrm{ZnS}$ phosphors. $\mathrm{Cu}^{+}$cannot take part in any light emission due to its $\mathrm{d}^{10}$ state and without any empty d-state. However, when $\mathrm{Cu}^{+}$is excited, a temporary $\mathrm{d}^{9}$ configuration $\left(\mathrm{Cu}^{2+}\right)$ splits the d-state into $t_{2}$ and $e$ states [176]. The green emission is due to the transition from coactivator defect level to the $\mathrm{Cu}^{2+} t_{2}$ state. In the case of $\mathrm{Cl}$ as the coactivator, the excited $\mathrm{Cu}^{+}$and $\mathrm{Cl}^{-}$form a ion pair as shown in Figure 5.13(a).

The donor-acceptor pairs of green luminescent centers were confirmed by Suzuki and Shionoya through the polarization measurements $[93,177]$. Thus the recombination mechanism of green emission is the donor-acceptor type as illustrated in Figure 5.13(b). Free electrons generated during the excitation process travel through the lattice and are trapped in the donor impurity level while free holes are trapped in the acceptor impurity level. When excited donor and acceptors are close enough, recombination takes place and green 


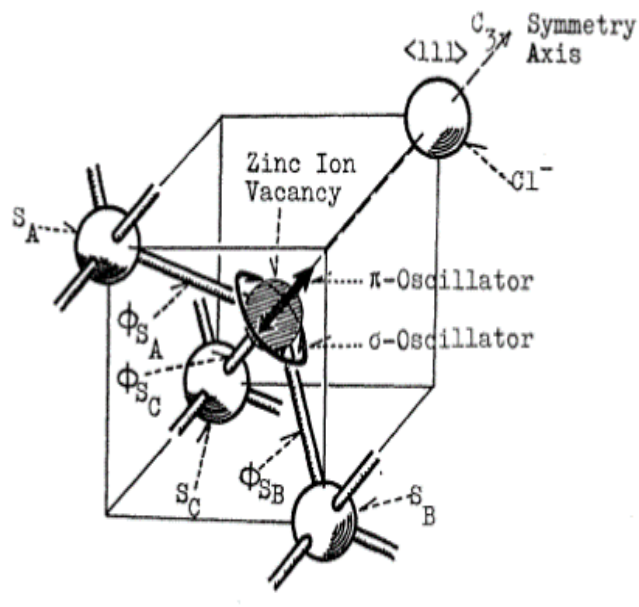

(a)

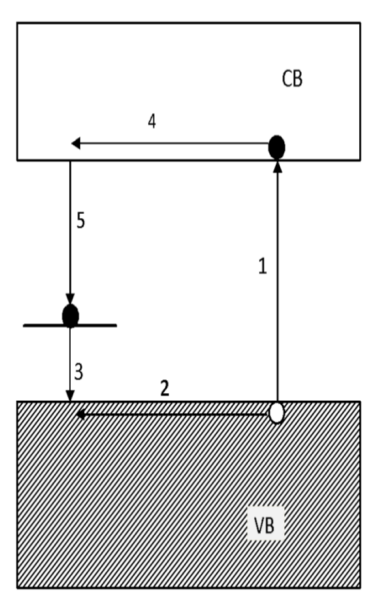

(b)

Figure 5.14: (a) Configuration of SA luminescent center in the zinc sulphide cubic cell caused by $\mathrm{C}_{3 \mathrm{~V}}$ structure [178]; (b) band diagram illustrating Schö-Klasens recombination mechanism [179].

light is emitted. The recombination probability follows the relationship:

$$
\beta_{g}=\beta_{g, \max } \exp \left(-r / R_{D}\right)
$$

where $R_{D}$ is the half Bohr radius of the shallower hydrogenic state. From Equation 5.8, it is noted that the recombination rate of green emission is determined by the distance $r$ of the $\mathrm{CuCl}$ ion pairs. This distance is not affected by the low operating frequency, which is in $\mathrm{kHz}$ scale. However, $r$ is subjected to lattice relaxation, which is temperature dependent.

\section{SA luminescent center}

As shown in Figure 5.12, with excess coactivators, blue emissions due to SA luminescent centers are generated. Actually, even without $\mathrm{Cu}$ addition, $\mathrm{SA}$ luminescence was also noticed in $\mathrm{ZnS}: \mathrm{Cl}$ phosphors [178]. Prener and williams proposed that this SA luminescent center consists of a zinc vacancy with an impurity such as $\mathrm{Cl}$ associated at a nearest neighbour site, which is equivalent to a $\mathrm{Cu}$ luminescent center site [180]. This is further confirmed by the polarization characteristics both in fluorescence and in thermolumines- 
cence in cubic $\mathrm{ZnS}: \mathrm{Cl}$ single crystals $[178,181]$. As illustrated in Figure 5.14(a), the SA luminescent center has an axial $\mathrm{C}_{3 \mathrm{v}}$ symmetry directed along the (111) axis. In the excited state, one electron excited from the sulfur ions surrounding the $\mathrm{Zn}$ vacancy resides in the $4 \mathrm{~s}$ orbital of the $\mathrm{Cl}$ ion and the hole left behind is supposed to be rotating among the three equivalent sulfur ions around the pair axis. This exciton configuration is similar to selftrapped exciton (STE), which is particularly common in metal halide and rare-gas crystals [182]. STE is due to the strong coupling of electrons or holes to the crystal lattice. In the lattice distortion field due to vacancies or impurities, a carrier may be self-trapped as a small polaron $[183,184]$.

The SA STE configuration further acts as the basis for the proposed Schön-Klasens Model of SA recombination mechanism as shown in Fig. 5.14(b). During electroluminescence, free electrons and holes are generated at a distance away from luminescent centers (step 1). Free holes may migrate toward the impurity center (step 2) and may be captured to form SA luminescent center (step 3). Free electrons wander through the lattice until they come close to the center (step 4) and are captured by the center with blue emissions (step 5). Thus the SA luminescence transition is a monomolecular process with a decay constant of around $0.4 \mu \mathrm{s}$, compared with $500-800 \mu \mathrm{s}$ for $\mathrm{G}-\mathrm{Cu}$ luminescence at room temperature [185].

\section{$B$-Cu luminescent center}

The B-Cu luminescent center was proposed by Blick et al. [186] to be formed by an adjacent substitutional and interstitial $\mathrm{Cu}^{+}$pair. This hypothesis was later proven wrong from the EXAFS study of $\mathrm{ZnS}: \mathrm{Cu}$ nanocrystals, which evidently indicates that no more than $2 \%$ of the $\mathrm{Cu}$ are in interstitial sites [66]. Later, Urabe et al. [94] confirmed that the B-Cu is associated with a complex center more like a $\mathrm{C}_{3 \mathrm{v}}$ symmetry. This finding agrees well with the fact that isolated $\mathrm{Cu}$ at substitutional zinc sites do not give rise to a luminescent center in $\mathrm{ZnS}$ as revealed from the radioative decay of $\mathrm{Zn}^{65}$ by Prener and Williams [174]. Car 


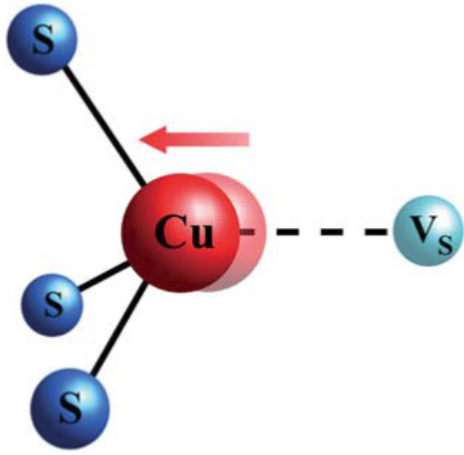

(a)

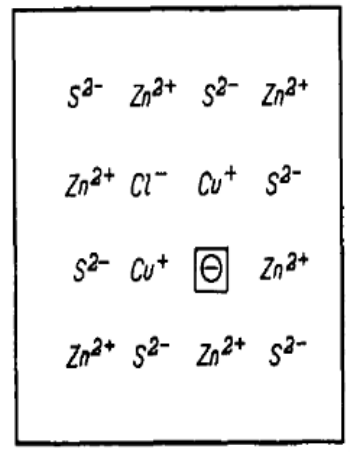

(b)

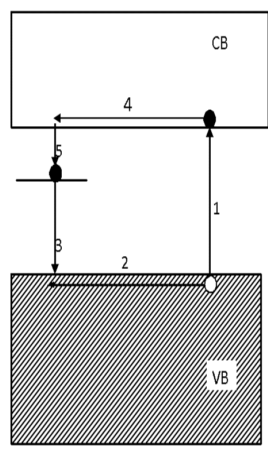

(c)

Figure 5.15: (a) The local environment about $\mathrm{CuS}$ in $\mathrm{ZnS}$ showing the $\mathrm{Cu}$ (red) displaced away from a $\mathrm{S}$ vacancy (light blue) and towards the three remaining $\mathrm{S}$ neighbours (blue) [66]; (b) local atomic arrangement of B-Cu luminescent center with $\mathrm{S}$ vacancy and $\mathrm{Cu}_{2} \mathrm{Cl}$ substitutions [171]; (c) Lambe-Klick model for the B-Cu recombination mechanism [187].

et al. probed the local structure of $\mathrm{ZnS}: \mathrm{Cu}$ nanocrystals and found that $\mathrm{Cu}$ has a nearest neighbouring $\mathrm{S}$ vacancy with the number of $\mathrm{S}$ neighbours below 4 (3.2). The $\mathrm{Cu}-\mathrm{S}$ distance is also found to be around 0.07-0.08 $\AA$ shorter than the $\mathrm{Zn}-\mathrm{S}$ distance, which arises from a [111] displacement of $\mathrm{Cu}$ away from the vacancy and towards the three $\mathrm{S}^{2-}$ as shown in Figure 5.15(a). As mentioned earlier, there is no $\mathrm{Cu}$-blue emission in $\mathrm{ZnS}$ phosphors without coactivators, which is different in micropowders from nanocrystals. Krger et al. found that the amount of $\mathrm{Cu}$ in $\mathrm{B}-\mathrm{Cu} \mathrm{ZnS}$ phosphors is around two times than that of $\mathrm{Cl}$ and therefore the $\mathrm{B}-\mathrm{Cu}$ luminescent center was proposed to be a complex structure containing two $\mathrm{Cu}^{+}$, one $\mathrm{Cl}^{-}$and a $\mathrm{S}$ vacancy as shown in Figure 5.15(b).

The recombination mechanism of $\mathrm{B}-\mathrm{Cu}$ luminescence was proposed to follow the LambeKlick model as concluded from the different decay characteristics of luminescence and photoconductivity [187]. This was confirmed by several research works. Arbell and Halperin found that the position of the glow current peak does not agree with the B-Cu glow peak [188]. Moreover, the excitation spectrum for the B-Cu luminescence does not agree with that for photoconductivity at low temperatures but becomes to agree with increase in temperature [189]. From the temperature dependencies of the peak positions and half-widths of the emission spectra, Shionoya et al. also suggests that the recombination of $\mathrm{B}-\mathrm{Cu}$ 
luminescence follows the Lambe-Klick model [77]. The schematic band diagram of the Lambe-Klick model is shown in Fig. 5.15(c). With free charged generated by electric fields (step 1), free electrons and holes move in their respective bands (step 4 and 2). Holes migrate near the impurity center and are captured by the complex center and B-Cu emission occurs (step 3). Electrons are then captured by the complex center (step 5). This model explains the slower photoconductivity decay of B-Cu emission. However, Urabe et al. concluded that B-Cu luminescence is not supported by Lambe-Klick model due to the lower symmetry of B-Cu center than the host lattice [94]. Era et al. observed that there is no spectrum shift during decay and with excitation intensity for $\mathrm{B}-\mathrm{Cu}$ in $\mathrm{ZnS}: \mathrm{Cu}, \mathrm{Cl}$ (or I) and suggested that the recombination mechanism is not donor-acceptor pair emission type [190]. Instead, the B-Cu is proposed to be due to the transition of intra-center type tightly associated center. Another infrared emission study by Matsuura and Tsurumi indicated the presence of a hole trap very close to valence band for the B-Cu luminescence [191]. From the above reviews, it is reasonable to propose that the $\mathrm{B}-\mathrm{Cu}$ luminescence transition is still a Lambe-Klick type but holes in step 2 fall into the deep traps much closer to valence band within the B-Cu luminescent complex center. With the close distance between these traps within the complex center, free electrons in the shallow traps recombine with trapped holes with overcoming only minimal energy barrier.

\subsubsection{Charge transport and relaxation model}

As far, the luminescence centers and recombination mechanisms of $\mathrm{G}-\mathrm{Cu}, \mathrm{SA}$, and $\mathrm{B}-\mathrm{Cu}$ have been reviewed and determined. However, the positions of these three centers are still unknown. A HTEM investigation of the twin boundary in $\mathrm{ZnS}: \mathrm{Cu}$ crystals by Guo et al. indicates that the twin boundary area and crystal streaks have higher $\mathrm{Cu}$ concentrations [169]. This high $\mathrm{Cu}$ content is necessary for $\mathrm{B}-\mathrm{Cu}$ luminescent center formation. Another degradation study conducted by Hirabayashi et al. [192] found similar lifetime trend between the concentration of S vacancy and (111) peak intensity. It is therefore postulated 
that the (111) twin boundary is also rich in S vacancy, which is part of the B-Cu luminescent center composition. A recent experiment on thermal doping found that the $\mathrm{B}-\mathrm{Cu}$ center is localized closer to the doping surface than G-Cu centers [193]. Therefore, in our proposed model, the B-Cu and SA luminescent centers are located near the grain boundary while G-Cu centers are located in the bulk $\mathrm{ZnS}$ grains.

Many research works have found that there are primary and secondary EL waves in the emission waveform within one single half cycle of the operating voltage $[48,168$, 194-201]. This is a strong indication of the separated process between excitation and recombination. The primary emission may act as the excitation for the secondary emission while the secondary emission excites the luminescent centers for the primary emission for the next cycle. This involves the building up of space charges within the $\mathrm{ZnS}$ grains, as suggested by Ptek [198]. The secondary waves are due to the reversed movement of charges upon removal of electric field. The intensity of primary and secondary waves is highly sample dependent. Moreover, both green and blue band emissions exist in primary and secondary waves [202]. It has also been found that the blue PL decay is much faster than the blue EL decay while the green PL decay is much slower than the green EL decay. This suggests that the excitation site is closer to the G-Cu luminescent centers. Free carriers need to overcome small barriers to reach B-Cu and SA centers [191, 203]. Therefore, the charge transport and relaxation model for frequency dependent color shift is proposed as follows.

A electron flux is generated during the voltage rise stage of the positive half cycle and is near the donor-acceptor pairs of G-Cu luminescent centers as shown in Figure 5.16(a). Within a certain time, free electrons hop to the donor-acceptor ion pair and are trapped in $\mathrm{Al}$ impurity center. G-Cu luminescence centers are filled during the last emission cycle and are subjected to charge relaxation. G-Cu luminescence occurs only when donor-acceptor are close enough. Excess electrons fill the empty Al sites. Some free electrons then migrate further toward SA centers and recombine with the unrelaxed holes with SA blue emissions. 


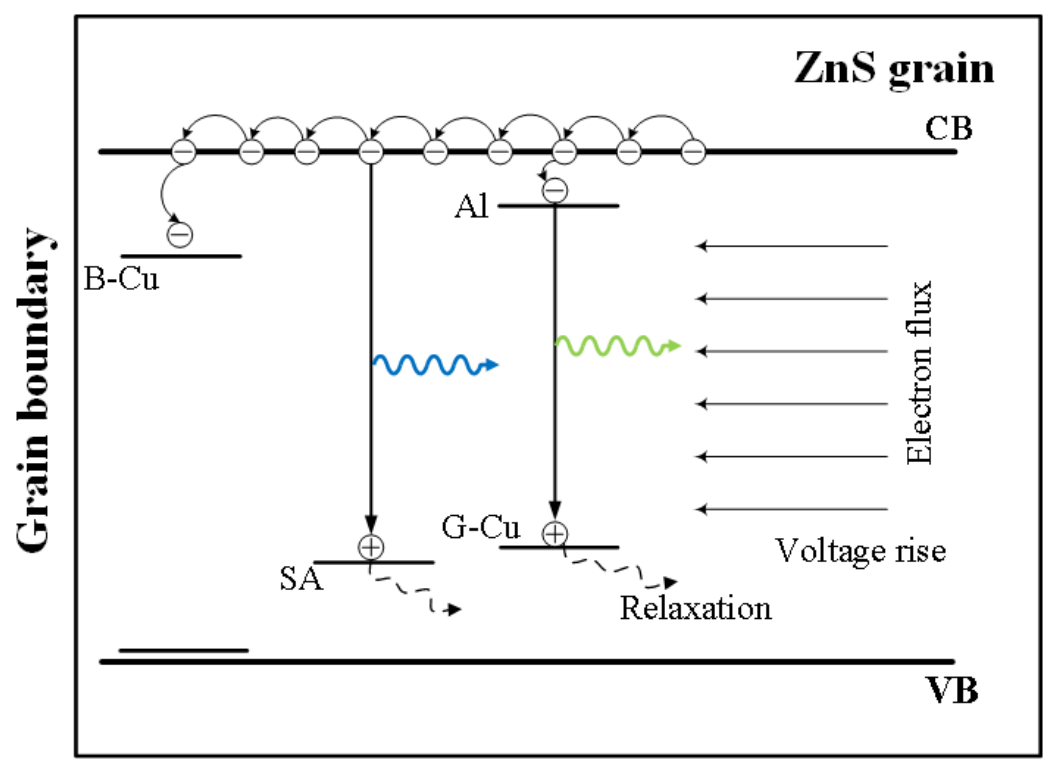

(a)

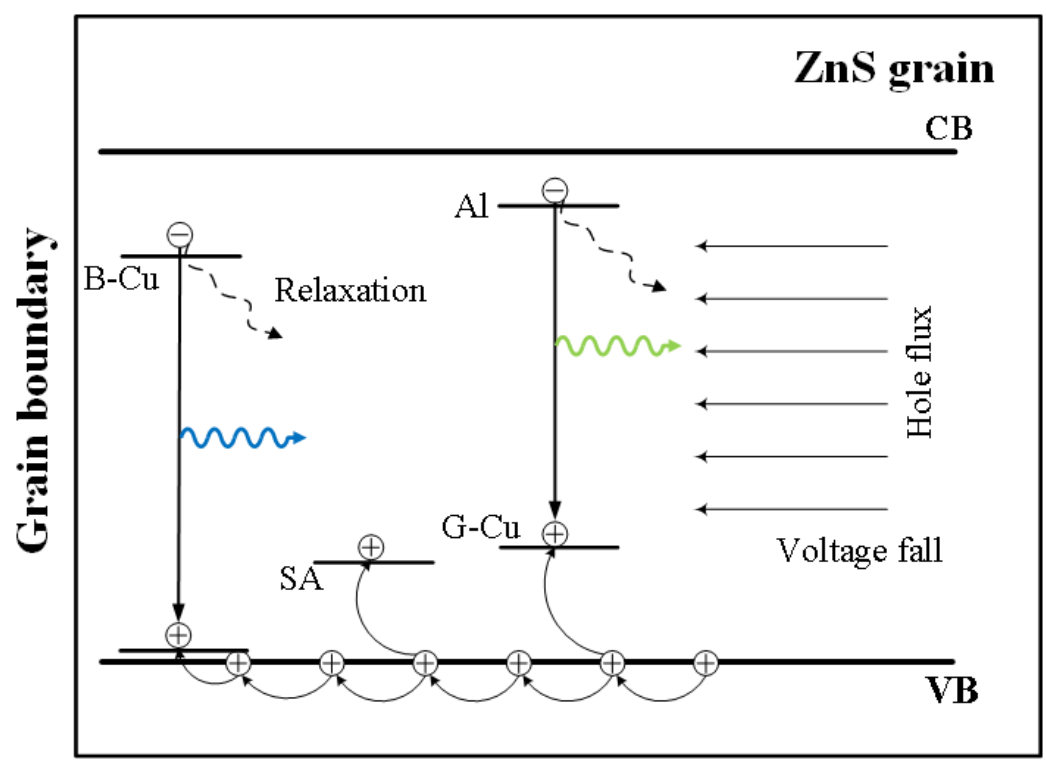

(b)

Figure 5.16: A charge transfer and relaxation model for the frequency dependent color shift behaviours associated with $\mathrm{G}-\mathrm{Cu}, \mathrm{SA}$ and $\mathrm{B}-\mathrm{Cu}$ luminescence centers during (a) voltage rise and (b) voltage fall of the positive half cycle.

Extra free electrons will travel further into the grain boundary and meet $\mathrm{B}-\mathrm{Cu}$ centers with certain probability of being trapped. In Figure 5.16(b), upon removal of the electric field, a hole flux is generated near the G-Cu luminescent centers. Some of the G-Cu impurity centers are activated by free holes and recombine with electrons trapped in $\mathrm{Al}$ impurity 
centers, which undergo a charge relaxation process. Emptied G-Cu luminescent centers are further filled by excess free holes. Some free holes migrate near the SA luminescent centers and are trapped there. Extra holes then hop over to the B-Cu complex center and trapped instantly in the hole traps very close to the valence band (VB), leading to the B-Cu luminescence. Due to the high detrapping rate, these shallow hole traps stay empty after recombination. This light emission process goes on for the next cycle.

\subsubsection{Analytical solutions}

$\mathrm{G}-\mathrm{Cu}$ EL intensity is the combination of that during voltage rise and voltage fall. During voltage rise, electron flux $\left(J_{n}\right)$ introduces a constant flux which is dependent on the voltage and temperature. Assuming the electron amount is enough for all the impurity centers, the amount of electrons migrate near G-Cu luminescent centers is:

$$
n_{G}=R_{G} \frac{t_{c}}{4}
$$

where $R_{G}$ is the amount of free electrons that reach G-Cu complex centers per unit time.

The traveling time for electrons is only $\frac{t_{c}}{4}$ due to the fact that the voltage rise occupies only one quarter of the operating cycle time $\left(t_{c}\right)$. Assuming no detrapping action from $\mathrm{Al}$ impurity center, the amount of trapped electrons in Al impurity centers becomes:

$$
n_{A l}=\alpha_{A l} n_{G} N_{A l}
$$

where $\alpha_{A l}$ is the electron trapping rate and $N_{A l}$ is the number of empty Al impurity sites. Assuming the number of excited G-Cu luminescent centers from the last voltage fall cycle to be $p_{G}$, after charge relaxation the intensity of green emission during voltage rise becomes:

$$
I_{G r}=\beta_{G} n_{A l} p_{G} e^{-\frac{t_{c}}{2 \tau_{G}}}
$$


During voltage fall, the amount of holes that migrate near G-Cu luminescent centers is also time dependent.

$$
p_{G}=S_{G} \frac{t_{c}}{4}
$$

where $S_{G}$ is the amount of holes that are able to reach G-Cu luminescent centers per unit time. With the consideration of charge relaxation of excited $\mathrm{Al}$ impurity centers, the $\mathrm{G}-\mathrm{Cu}$ luminescence intensity during voltage fall is:

$$
I_{G f}=\beta_{G} p_{G} n_{A l} e^{-\frac{t_{c}}{4 \tau_{A l}}}
$$

By adding Equations 5.11 and 5.13, the total intensity of G-Cu luminescence is therefore:

$$
I_{G}=A t_{c}^{2}\left[e^{-\frac{t_{c}}{2 \tau_{G}}}+e^{-\frac{t_{c}}{4 \tau_{A l}}}\right]
$$

where

$$
A=\frac{1}{16} \beta_{G} \alpha_{A l} \alpha_{G} R_{G} S_{G} N_{A l} N_{G}
$$

With similar approach, the intensities of SA and B-Cu luminescence in a cycle are calculated as follows.

$$
\begin{gathered}
I_{G}=B t_{c}^{2} e^{-\frac{t_{c}}{2 \tau_{S A}}} \\
I_{G}=C t_{c}^{2} e^{-\frac{t_{c}}{4 \tau_{B}}}
\end{gathered}
$$

where

$$
\begin{gathered}
B=\frac{1}{16} \beta_{S A} \alpha_{S A} R_{S A} S_{S A} N_{S A} \\
C=\frac{1}{16} \beta_{B} \alpha_{B} R_{B} S_{B} N_{B}
\end{gathered}
$$

where $\beta_{S A}$ and $\beta_{B}$ are the recombination probability of SA and B-Cu luminescence, $\alpha_{S A}$ and 
$\alpha_{B}$ the electron/hole trapping rate in $\mathrm{SA}$ and B-Cu impurity centers, $R_{S A}$ and $R_{B}$ the amount of electrons that reach near SA and B-Cu luminescent centers per unit time, $S_{S A}$ and $S_{B}$ the amount of holes that reach near SA and B-Cu luminescent centers per unit time, $N_{S A}$ and $N_{B}$ the amount of empty SA and B-Cu luminescent centers, $\tau_{S A}$ and $\tau_{B}$ charge lifetime in $\mathrm{SA}$ and B-Cu luminescent centers.

\subsection{Discussion}

To simulate the luminescence intensity in relation with different operating frequencies, Equations 5.14, 5.16 and 5.17 are converted to the following expressions by taking $t_{c}=1 / f$.

$$
\begin{gathered}
I_{G}=A\left(\frac{1}{f}\right)^{2}\left[e^{-\frac{1}{2 f \tau_{G}}}+e^{\left.-\frac{1}{4 f \tau_{A l}}\right]}\right. \\
I_{S A}=B\left(\frac{1}{f}\right)^{2} e^{-\frac{1}{2 f \tau_{S A}}} \\
I_{B}=C\left(\frac{1}{f}\right)^{2} e^{-\frac{1}{4 f \tau_{B}}}
\end{gathered}
$$

From Equations 5.20-5.22, A, B, and C are independent of frequency and are all taken as 1 . The $\mathrm{G}-\mathrm{Cu}$ luminescence intensity is thus varied with the lifetime of $\mathrm{G}-\mathrm{Cu}$ and $\mathrm{Al}$ impurity centers, which ranges from a few hundred microseconds to milliseconds. As shown in Figure 5.17(a), the intensity of G-Cu luminescence is plotted against frequency with varied Al impurity lifetime and fixed G-Cu lifetime at $1 \mathrm{~ms}$. At $100 \mu \mathrm{s}$ and $1 \mathrm{~ms}$, there are small peaks at low frequencies, which disappear with Al lifetime at 2 ms onwards. Moreover, the G-Cu luminescence intensity increases with Al lifetime. With longer lifetime, charges do not have enough time to migrate near G-Cu impurity centers with increased frequency and thus the luminescence intensity drops. At low frequency, the charge relaxation dominates. With longer lifetime, more charges survive for the recombination provided the 


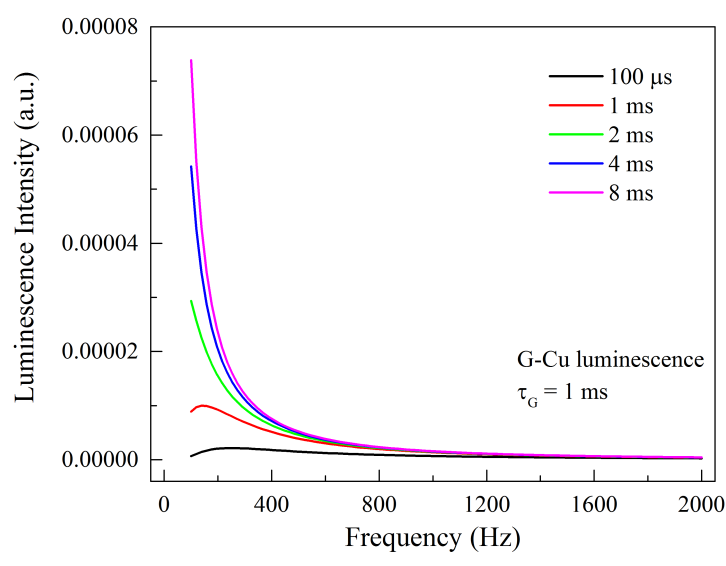

(a)

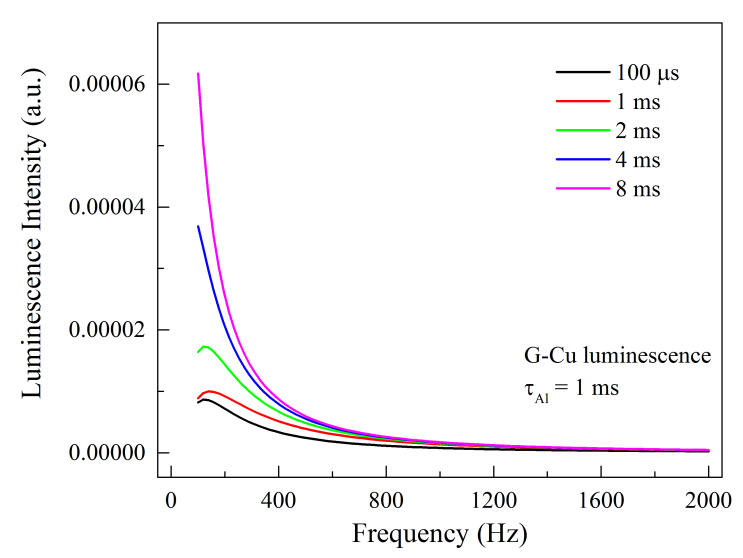

(b)

Figure 5.17: G-Cu luminescence intensity as a function of frequency with different charge lifetimes in (a) $\mathrm{Al}$ and (b) G-Cu impurity centers.

excited electron/hole flux is not changed, leading to higher luminescence intensity. With the $\mathrm{Al}$ lifetime fixed at $1 \mathrm{~ms}$, the change of intensity spectra as a function of frequency at different G-Cu lifetimes in Figure 5.17(b) is similar to that in Figure 5.17(a). Due to the factor of 4 associated with $\mathrm{Al}$ compared to a factor of 2 with G-Cu in Equation 5.20, the effects of $\mathrm{Al}$ lifetime are larger than that of G-Cu lifetime on luminescence intensity. The simulated results are well fitted with the experimental results for G-Cu emission in Section 5.3 for both green and blue ACPEL devices.

The luminescence intensity as a function of frequency for SA and B-Cu luminescence is plotted in Figure 5.18. At short lifetime, the intensity spectrum is similar with the results shown in Section 5.3 for both green and blue devices. Namely, the intensity per cycle increases first with frequency and slowly decreases thereafter for both SA and B-Cu luminescence. In the current simulation, this is the case only when SA and B-Cu luminescent centers have short lifetime, which is observed experimentally [204]. At longer lifetime, the luminescence peak is intensified and shift to lower frequency.

From this charge transport and relaxation model for the successful explanation of frequency dependent color shift, it is possible to understand the low luminous efficacy of ACPEL devices. Similarly with SA and B-Cu luminescence, G-Cu luminescence is sub- 


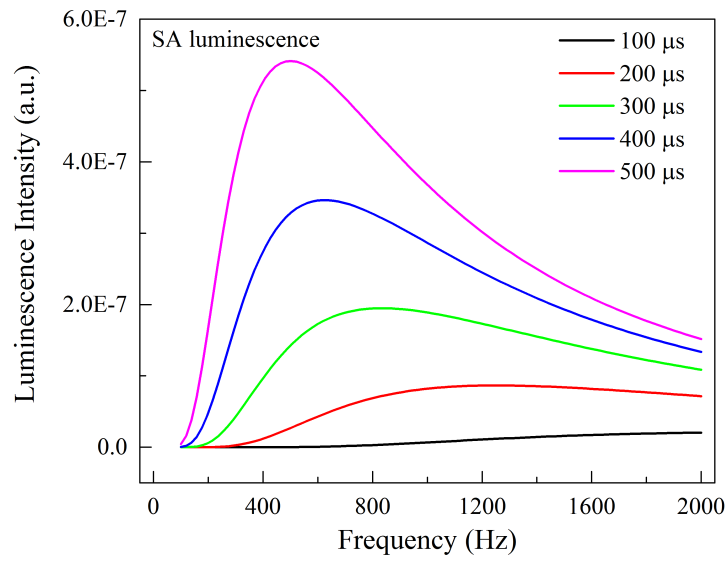

(a)

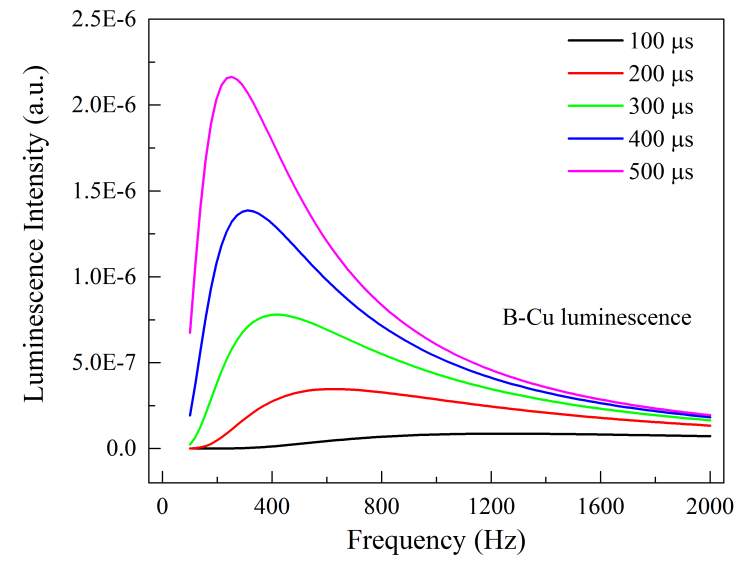

(b)

Figure 5.18: (a) SA and (b) B-Cu luminescence intensity as a function of frequency with different charge lifetimes in SA and B-Cu impurity centers.

jected to the various loss as shown in Equation 5.15. Even with the assumption of fully utilization of excited charges, the efficiency is equal to:

$$
\eta=\frac{I_{G}+I_{S A}+I_{B}}{1 / 4 J_{n} t_{c}+1 / 4 J_{p} t_{c}}
$$

From the point of $\mathrm{ZnS}$ phosphor level, the power efficiency is thus determined by the recombination rate, electron and hole trapping rate, and the lifetime of impurity centers. The possible loss of charges are due to the insufficient recombination, insufficient utilization of charges for trapping actions and the loss of charges during the relaxation of excited impurity centers.

\subsection{Conclusion}

In this chapter, the frequency dependent color shift phenomenon is studied experimentally and theoretically. The EL emissions of green and blue EL films are found to be composed of three emission lines, corresponding to $\mathrm{G}-\mathrm{Cu}, \mathrm{B}-\mathrm{Cu}$ and SA emissions. With the indepth study of the literature, the luminescent center of $\mathrm{G}-\mathrm{Cu}$ is $\mathrm{Cu}-\mathrm{Cl}$ ion pair while the $\mathrm{B}-\mathrm{Cu}$ and $\mathrm{SA}$ luminescence are associated with $\mathrm{Cu}_{2} \mathrm{Cl}-\mathrm{V}_{\mathrm{S}}$ and $\mathrm{V}_{\mathrm{Zn}}-\mathrm{Cl}$ complex centers. 
The recombination mechanisms for these three emissions belong to DA, Schön-Klasens and Lambe-Klick types. A charge transport and relaxation model is built and the simulated results well fit the trend of $\mathrm{G}-\mathrm{Cu}, \mathrm{B}-\mathrm{Cu}$ and SA emission peaks with frequency. A insight explanation is thus provided for the low luminous efficacy of ACPEL devices. 


\section{Chapter 6}

\section{TEMPERATURE-DEPENDENT IMPEDANCE SPECTROSCOPY OF ACPEL DEVICES}

\subsection{Introduction}

In the previous chapters, various low cost printing processes were employed to extend the color selections of printed EL films. Large area white and warm white hybrid EL films were achieved. The frequency-dependent color changing characteristics were observed and investigated experimentally and theocratically. However, the luminous efficacy of these hybrid devices was very low compared to LEDs. It has been shown in Chapter 5 that one of the attributions to the low luminous efficacy is the poor luminance conversion efficiency in ZnS phosphor due to the charge relaxation of the excited impurity centers and the limited trapping and recombination probabilities. The other aspect of such low luminous efficacy may lies in the electrical loss within the capacitor like ACPEL structures. Therefore, it is worthwhile to understand the electric/dielectric properties and the equivalent circuit of ACPEL devices. ACPEL devices are generally assumed to be capacitive by nature, borne out from a resistor (R) and capacitor (C) circuitry. Various simplified RC-based equivalent circuit models have been proposed. Bredol and Dieckhoff demonstrated that ACPEL devices could be represented by a capacitive load with series and shunt resistors [34]. The shunt and series resistors represent the direct current (DC) conductivity of ACPEL devices and resistivity of the contacts, respectively. Winscom et al. proposed an equivalent circuit model with effective $\mathrm{R}$ and $\mathrm{C}$ components in a series configuration [205]. They suggested that the effective capacitance was governed by the dielectric material and the phosphor particles were accountable for the effective resistance in the single-layer ACPEL devices. However, the equivalent circuit for ACPEL devices is more complex with the consideration 
of the microscopic features and interfaces [116].

An ACPEL device is a complex composite system with several stacked polymer composite layers. A dielectric layer is typically printed over a light emitting layer, which typically contains $\mathrm{ZnS}$ phosphor microparticles to increase the electrical field surrounding the $\mathrm{ZnS}$ microparticles during electrical excitation and, in turn, increase its light emission properties. The incorporation of micro- or nanofillers into a polymeric material will introduce interfacial polarization or MWS effect at the particle/matrix interfaces and enhance the dielectric performance of the polymer composite material [206, 207]. In this chapter, complex impedance spectroscopy was employed to obtain the dielectric properties of ACPEL devices. In order to segregate the types of relaxation processes in the device, the dielectric properties of the composite films containing either a dielectric layer or a light emitting layer and containing only binder were also measured and compared with those of ACPEL devices. From the analysis of impedance data, equivalent circuit models were subsequently proposed for different printed device structures.

\subsection{Experimental methods}

\subsubsection{Fabrication of samples for impedance spectroscopy}

Due to the complexity of the stacked composite layers in ACPEL devices, only the forward devices were used so as to eliminate the effect of PEDOT:PSS layer. The fabrication process for forward ACPEL devices with the structure in Figure 6.1(a) is listed in Chapter 3. To investigate the dielectric relaxation process in individual layers, a phosphor film and a dielectric film containing only a layer of $\mathrm{ZnS}$ microcomposites or $\mathrm{BaTiO}_{3}$ nanocomposites between Ag and ITO electrodes as shown in Figures 6.1(b) and 6.1(c) were fabricated using the same method as the forward ACPEL devices in Section 3.2.1. To enable the understanding of the dielectric properties of the binder, a binder film (IBI) with the structure of Figure 6.1(d) was made by sandwitching a binder layer between two ITO-coated PET films. The binder layer was coated by drop casting sufficient amount of binder solution 


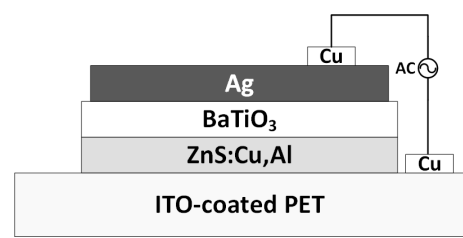

(a)

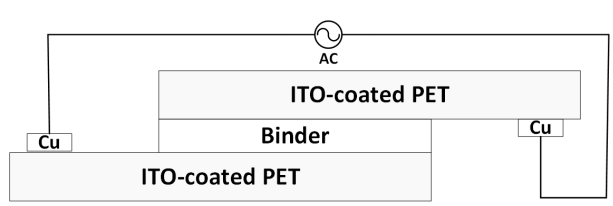

(d)

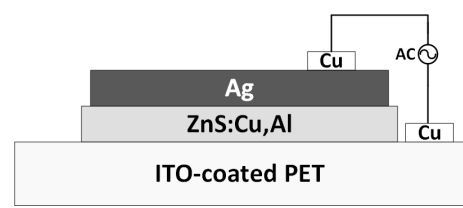

(b)

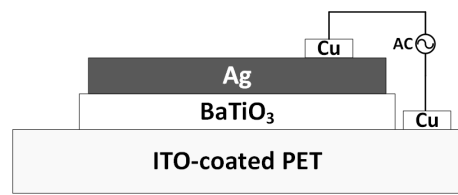

(c)

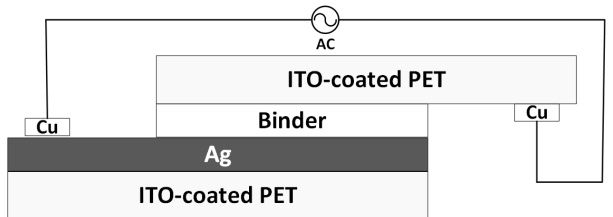

(e)

Figure 6.1: Schematic structures of (a) ACPEL devices, (b) a phosphor film, (c) a dielectric film, (d) a binder film with ITO electrodes, and (e) a binder film with one ITO and one Ag electrode used in the temperature dependent impedance spectroscopy study.

over an ITO-coated PET film. With the other ITO-coated PET film placed on top, the device went through an annealing process at $130^{\circ} \mathrm{C}$ for $30 \mathrm{~min}$ and the upper PET film was attached to binder firmly by gravity force. To minimize the possibility of short circuiting, the overlapped region between two PET substrates was filled by the drop-casted binder. Another binder-Ag film (IAgBI) with the structure of Figure 6.1(e) was also fabricated in order to study the dielectric contributions of the Ag electrode. The Ag layer was screenprinted on an ITO-coated PET film using the printing speed of $40 \mathrm{~mm} / \mathrm{s}$ and annealed at $130{ }^{\circ} \mathrm{C}$ for $30 \mathrm{~min}$. This Ag film was then placed over an ITO-coated PET film deposited with a layer of drop-casted binder and annealed also at $130{ }^{\circ} \mathrm{C}$ for $30 \mathrm{~min}$. Two copper stripes were attached to all the fabricated samples to enable the electrical connection and dielectric spectroscopy measurements.

\subsubsection{Setup for temperature controlled impedance spectroscopy}

The experiment setup for studying the impedances at elevated temperatures is illustrated in Figure 6.2. The device under test (DUT) was placed in a oven with temperatures controlled from 50 to $150{ }^{\circ} \mathrm{C}$ with electromagnetic interference (EMI) shielded. A Autolab 


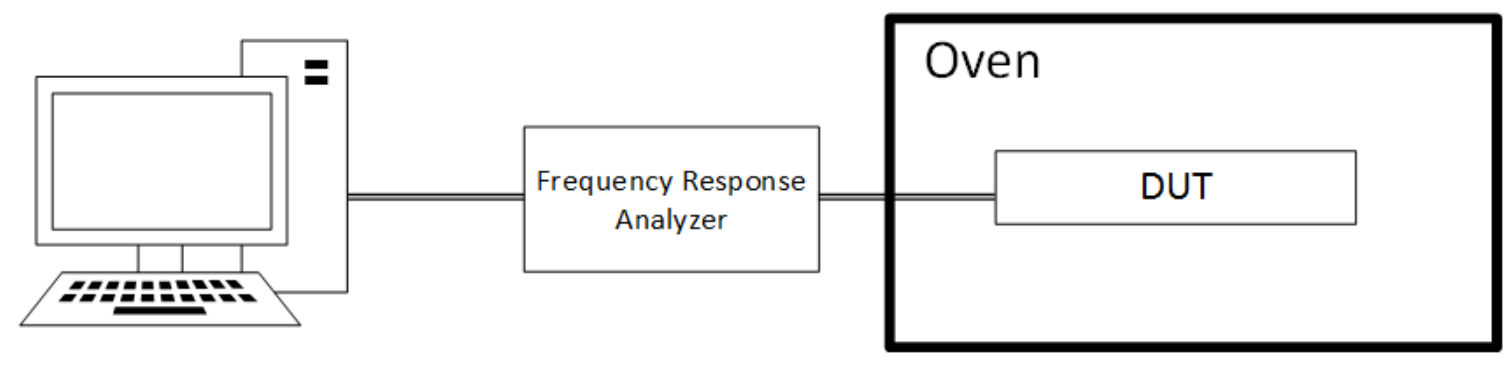

Figure 6.2: Schematics of the experiment setup for the temperature dependent impedance spectroscopy study of ACPEL devices.

PGSTAT302N was electrically connected to the DUT for impedance measurement which is equipped with a frequency response analyzer (FRA) FRA2 module having a current range from $10 \mathrm{nA}$ to $10 \mathrm{~mA}$ with resolution of $0.0003 \%$ and a potential resolution of $3 \mathrm{~V}$. The FRA was computationally controlled by Nova software, where the dielectric data was acquired and the corresponding equivalent circuit was simulated. A voltage signal with the peak value of $0.5 \mathrm{~V}$ and frequency ranging from $10^{-2}$ to $10^{5} \mathrm{~Hz}$ with a resolution of $0.0003 \%$ was supplied by the FRA and the corresponding current was measured. 50 data points were acquired over the frequency range. From the measured data, $Z$ ', $Z$ ”, and $\delta$ were acquired and computed automatically by the software. Other parameters like $\varepsilon^{\prime}, \varepsilon^{\prime \prime}, M^{\prime}$, $M ”$ and $\sigma^{\prime}$ were calculated according to Equations 2.42-2.47.

\subsection{Results and discussion}

\subsubsection{Temperature dependent relative Permittivity}

The relative permittivity $\varepsilon^{*}$ contains real and imaginary parts $\left(\varepsilon^{\prime}\right.$ and $\left.\varepsilon^{\prime}\right) . \varepsilon^{\prime}$ is also known as dielectric constant, which represents a material's ability to store energy in an electric field. $\varepsilon "$ is the dielectric loss, which stands for the energy loss in the material. The dielectric properties at different frequencies are called dielectric dispersion. Therefore, The change of permittivity as a function of frequency is called permittivity dispersion. The permittivity dispersion of ACPEL devices is shown in Figure 6.3. In Figure 6.3(a), $\varepsilon^{\prime}$ increases with the decrease of frequency. The $\varepsilon^{\prime}$ dispersion can be divided into three regions, 
corresponding to three time constants with three different polarization mechanisms. At low frequency, the near linear increase of $\varepsilon$ ' with the decrease of frequency corresponds to the electrode polarization (EP). If no EP is at the electrodes, electric neutrality shall be maintained and thus there should be a flat dispersion at low frequency. Therefore, a space charge layer is formed at the electrode with charges accumulated at the electrode/binder interfaces, leading to the increased $\varepsilon^{\prime}$ at low frequency. In fact, the dielectric and phosphor film together with IBI all exhibit similar trend as shown in Figures 6.4-6.6. This suggests that the ITO electrode is one of the charge accumulation sites and the permittivity dispersion is primarily the behavior of polymeric binders. As temperature increases, $\varepsilon^{\prime}$ increases in all cases, which suggests the increased polarizability of materials within binder, phosphor or dielectric materials.

In Figure 6.3(b), the $\varepsilon$ " dispersion also increases with the decrease of frequency at low frequency, similar to the $\varepsilon^{\prime}$ dispersion as shown in Figure 6.3(a). At low frequency, the inversely proportional relationship between $\varepsilon$ " and frequency indicates the existence of DC conductivity [208]. The similar $\varepsilon$ " dispersions in dielectric film, phosphor film and IBI show that this DC conductivity is mainly caused by the nature of binder. It has been demonstrated that in polyamide ionic carriers the internal defects introduced in the fabricating process are responsible for this DC conductivity [209]. $\varepsilon$ " also sees a increase with temperature indicating the increased energy loss. In Figure 6.4(b), there are clearly three regions with three different time constants corresponding to three different polarization processes. For IBI, there are only two regions corresponding to the two polarization mechanisms, one in binder and the other at the electrodes. Similarly, there are only two clear regions for ACPEL devices and phosphor film as shown in Figure 6.3(b) and 6.5(b). However, there is a small shoulder in ACPEL devices, which is probably due to another polarization process.

In Figure 6.7, $\varepsilon^{\prime}$ or $\varepsilon "$ for ACPEL devices, dielectric film, phosphor film and IBI is plotted into a single graph for better comparison. In Figure 6.7(a), $\varepsilon^{\prime}$ of IBI is smaller than 


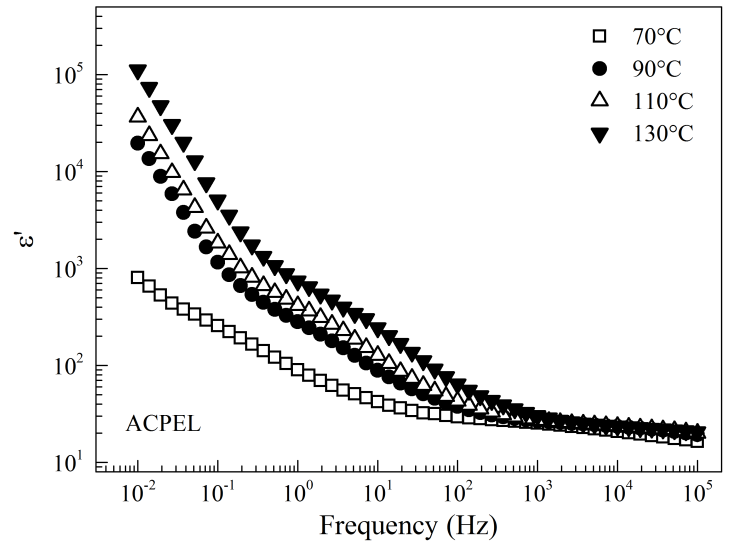

(a)

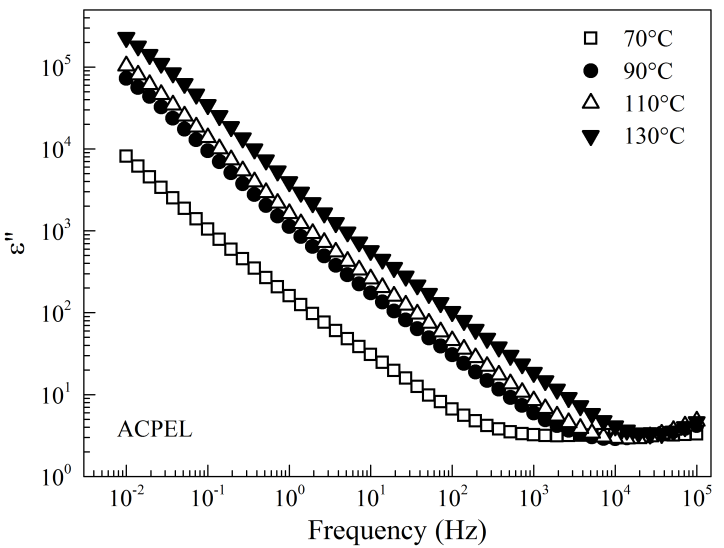

(b)

Figure 6.3: (a) $\varepsilon^{\prime}$ and (b) $\varepsilon$ " as a function of frequency at different temperatures for ACPEL devices.

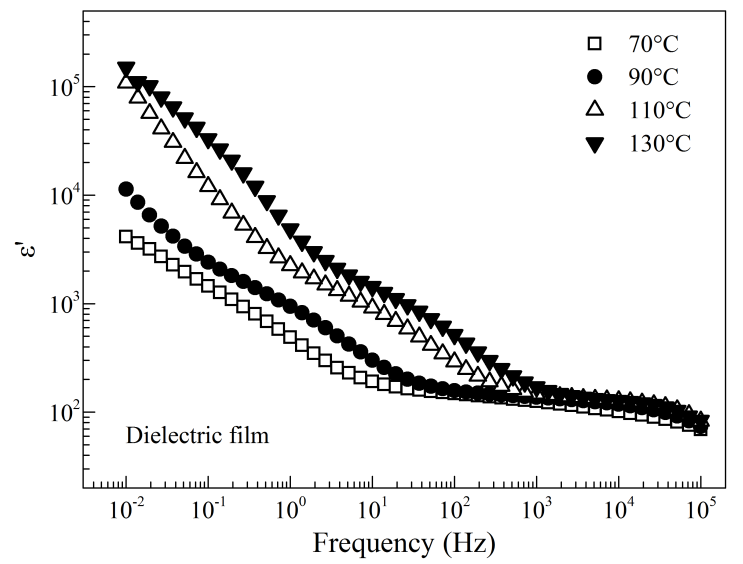

(a)

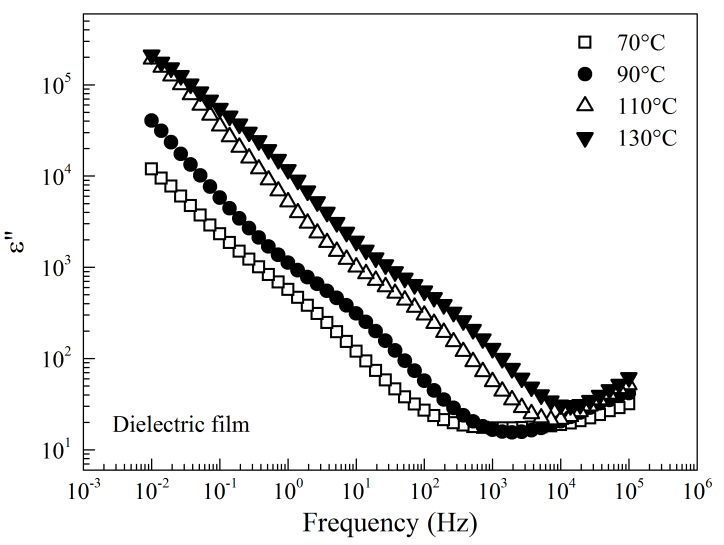

(b)

Figure 6.4: (a) $\varepsilon$ ' and (b) $\varepsilon$ " as a function of frequency at different temperatures for the dielectric film.

any of the other three samples. As in these three samples, micro- or nano-fillers are added into binder to form composites, leading to the increased $\varepsilon$ ' by introducing a commonly known MWS effect. However, the added fillers also cause more energy loss as shown in Figure 6.7(b), where $\varepsilon$ " of the composite devices is much higher than that of IBI. Moreover, the $\varepsilon$ ' or $\varepsilon$ " dispersion of ACPEL devices falls between that of the dielectric film and the phosphor film, which is more obvious at intermediate to high frequencies. This suggests that the dielectric properties of ACPEL devices may be the superposition of that of the 


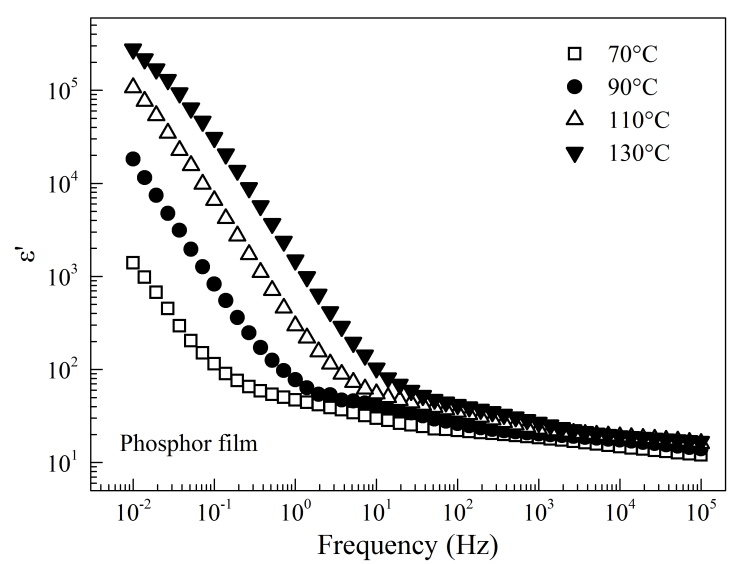

(a)

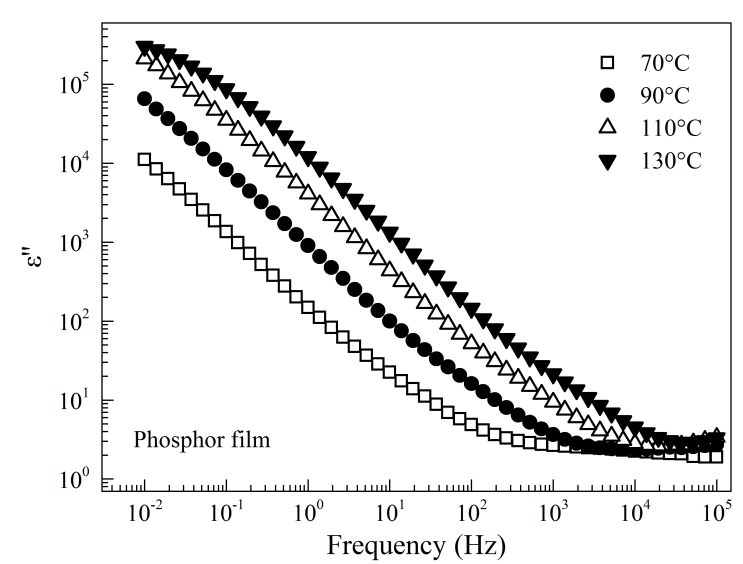

(b)

Figure 6.5: (a) $\varepsilon^{\prime}$ and (b) $\varepsilon "$ as a function of frequency at different temperatures for the phosphor film.

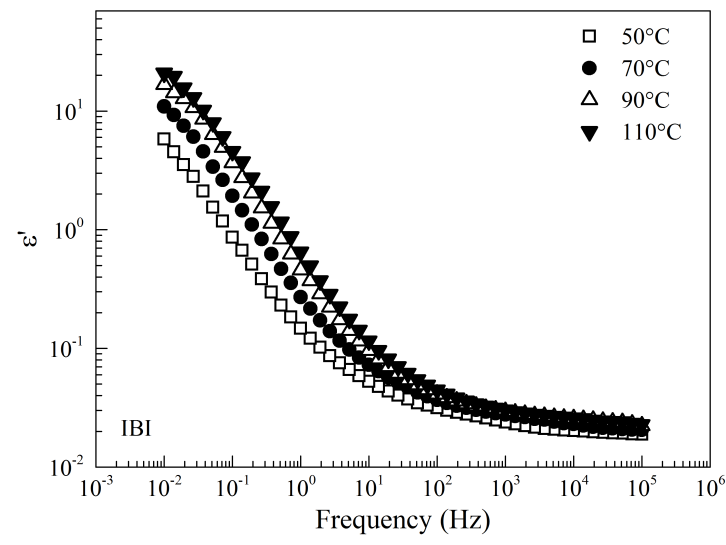

(a)

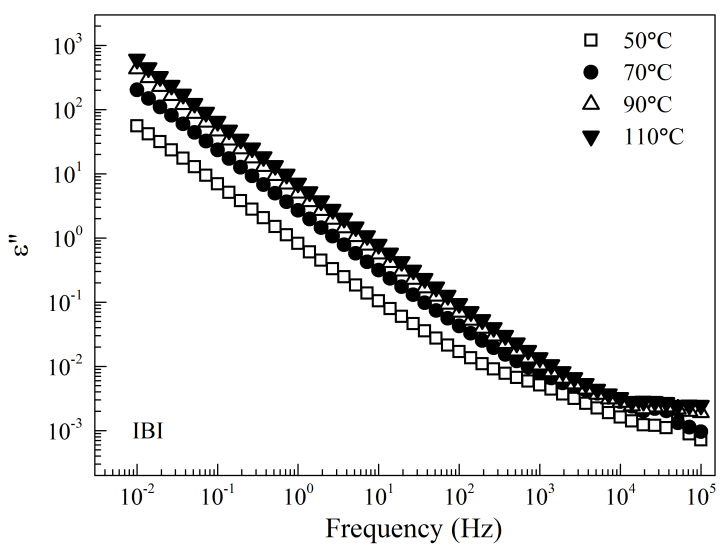

(b)

Figure 6.6: (a) $\varepsilon$ ' and (b) $\varepsilon$ " as a function of frequency at different temperatures for IBI.

dielectric film and phosphor film.

\subsubsection{Temperature dependent electric modulus}

Electric modulus $M^{*}$ is used to better represent the true relaxation processes in ACPEL devices, as it corresponds to the relaxation of electric field when the electric displacement remains constant [208]. The near zero values of $M^{\prime}$ in Figures 6.8-6.12 demonstrate the well removal of EP from ACPEL devices, dielectric film, phosphor film, IBI and IAgBI. In Figure 6.8(a), $M^{\prime}$ dispersion can be divided into two regions, one with bigger slope at 


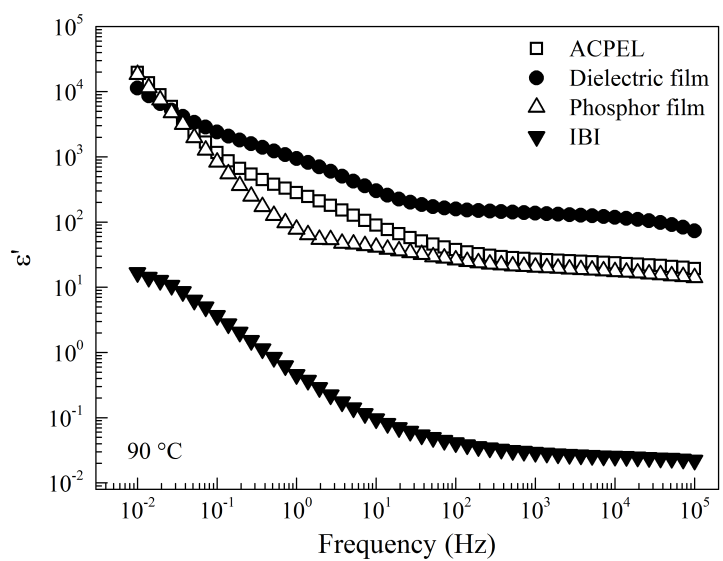

(a)

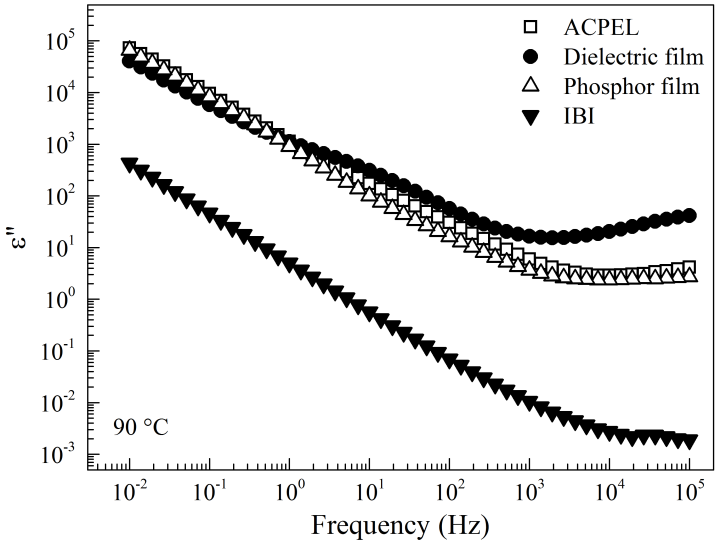

(b)

Figure 6.7: (a) $\varepsilon^{\prime}$ and (b) $\varepsilon$ " as a function frequency at $90{ }^{\circ} \mathrm{C}$ for ACPEL devices, dielectric film, phosphor film and IBI.

intermediate frequency and the other with smaller slope at high frequency. This suggests at least one time constant existing within ACPEL devices which are associated with one relaxation process. The similar $M^{\prime}$ dispersion trends in dielectric film, phosphor film, IBI and IAgBI are observed. This suggests that the relaxation process is originated within the binder. It has been shown that the high temperature relaxation process is attributed to the translational movement of polymer main chains [145], also known as $\alpha$ relaxation. When placed in one graph, the magnitude of $M^{\prime}$ in IBI in Figure 6.13(a) has been scaled down by a factor of 0.002 . The $M^{\prime}$ values for the ACPEL devices, dielectric film and phosphor film are much smaller than that of IBI. This indicates the addition of micro- or nano-fillers affects the dielectric properties of binder. With the $M^{\prime}$ values of ACPEL devices lying between that of the dielectric film and phosphor film, this further demonstrates that the dielectric property of ACPEL devices is the superposition of the two films.

The loss peaks of $M$ ” in Figures 6.8-6.12 further prove the existence of a $\alpha$ relaxation process in polymeric binders. It is also noticed that $M$ ” has the trend to further increase at high frequency for ACPEL devices, dielectric film and phosphor film. This tendency is not observed in IBI as shown in Figure 6.11(b). However, there is a small arc for IBI at high frequency. This arc may be due to the $\alpha$ relaxation of alternative polymeric phase in binder 


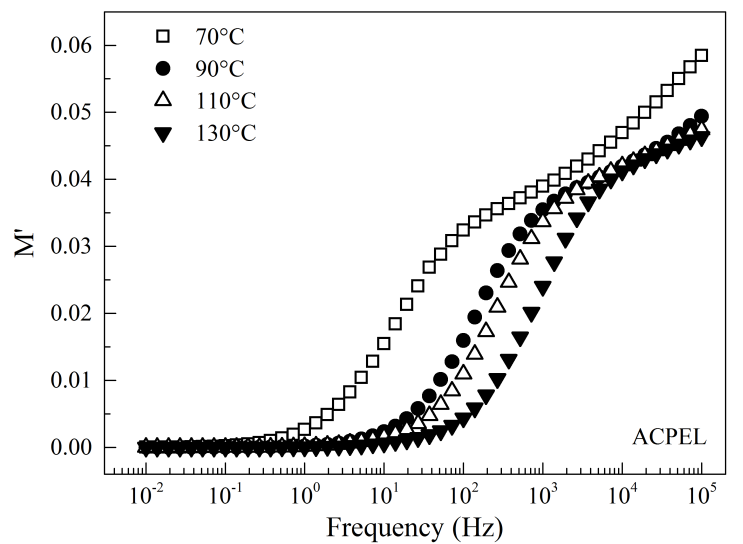

(a)

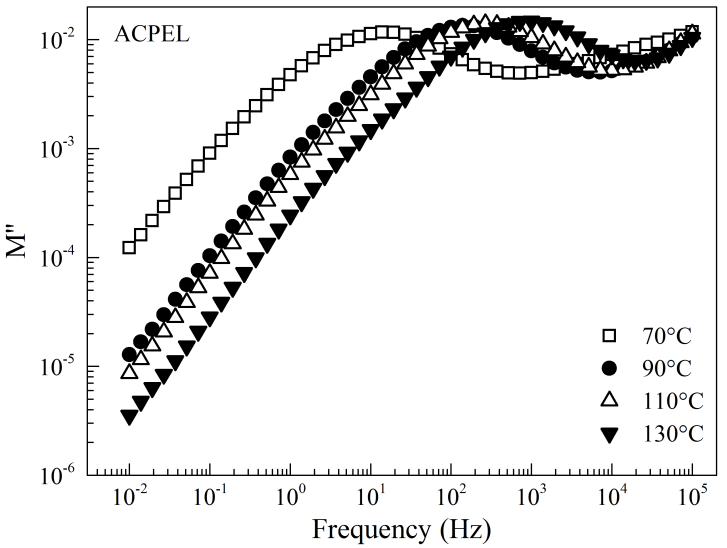

(b)

Figure 6.8: (a) $M^{\prime}$ and (b) $M^{\prime \prime}$ as a function frequency at different temperatures for the ACPEL devices.

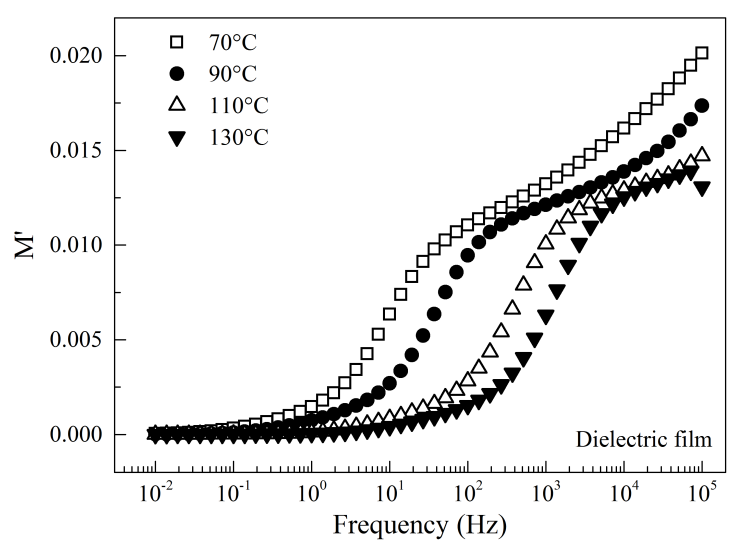

(a)

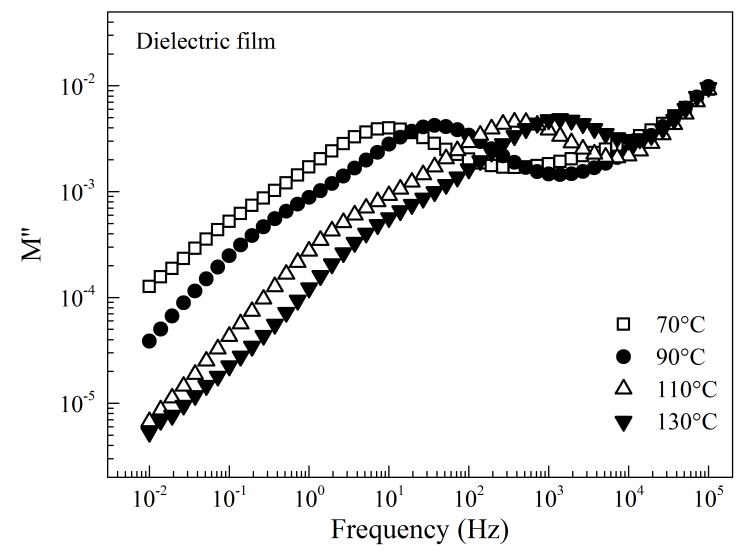

(b)

Figure 6.9: (a) $M^{\prime}$ and (b) $M^{\prime \prime}$ as a function frequency at different temperatures for the dielectric film.

or the relaxation of side chains with absorbed moisture [209], which we call B relaxation in this context. On the contrary, $M$ ” of IAgBI experiences a tendency to increase similarly with the ACPEL devices, dielectric film and phosphor film as illustrated in Figure 6.12(b). This tells us that the upward tendency at high frequency is due to the relaxation within $\mathrm{Ag}$ layer, which we call "A relaxation". This A relaxation at the nanoparticle electrode is due to the dipolar reorientation of the binder in electrode as proposed by Cha et al. [151].

In Figure 6.9(b), it is observed that there is a small shoulder at low frequency. With EP 


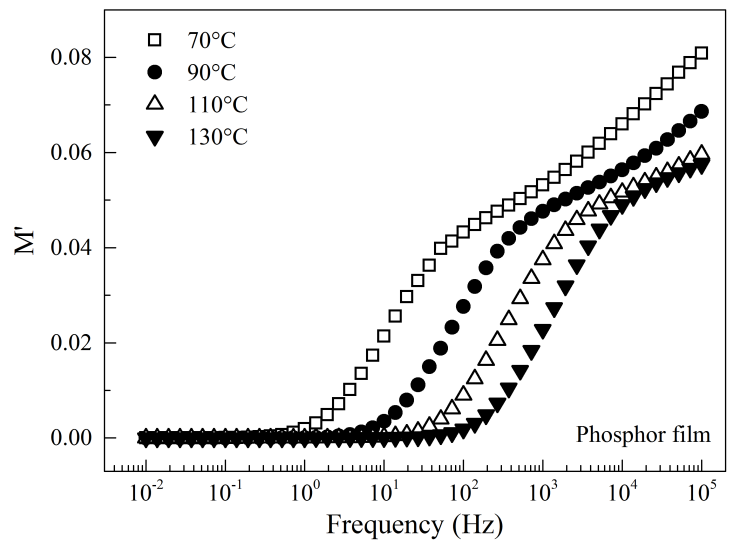

(a)

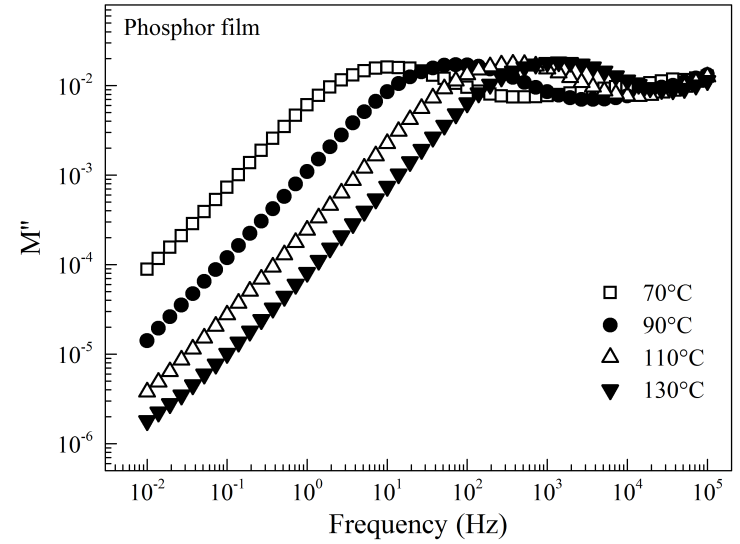

(b)

Figure 6.10: (a) M' and (b) M" as a function frequency at different temperatures for the phosphor film.

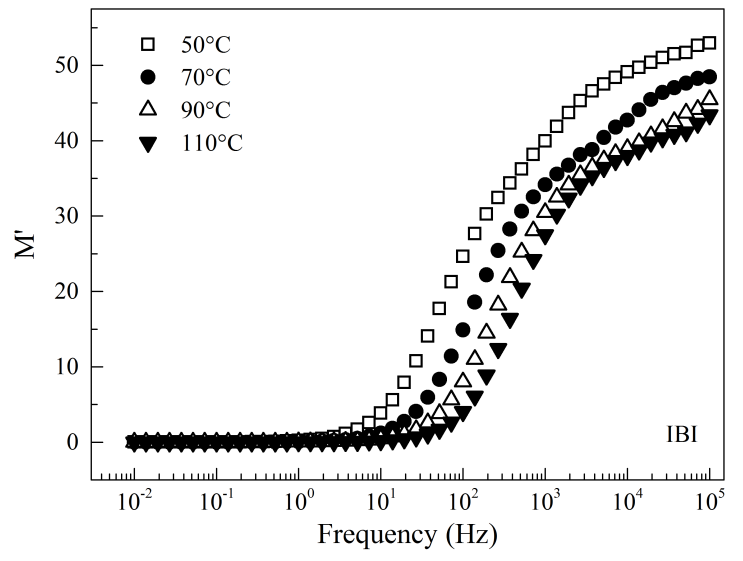

(a)

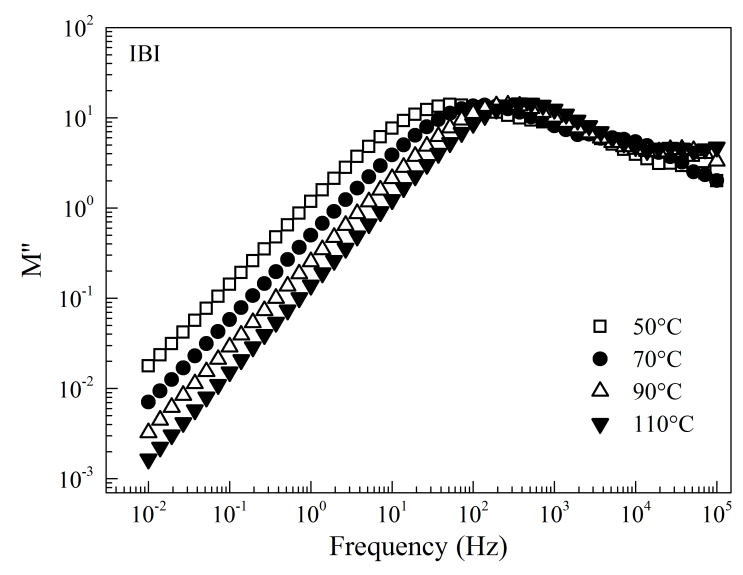

(b)

Figure 6.11: (a) M' and (b) M" as a function frequency at different temperatures for IBI.

suppressed, this shoulder is due to the MWS effect at the $\mathrm{BaTiO}_{3} /$ binder interface [210]. Because of the large permittivity for both binder and $\mathrm{BaTiO}_{3}$ nanoparticles, no obvious peak can be formed due to the MWS effect. At the $\mathrm{BaTiO}_{3} /$ binder interfaces, charges accumulate and large dipoles are formed with time constants larger than the $\alpha$ relaxation in binder. On the contrary, no shoulder is observed in the phosphor film as shown in Figure 6.10(b). As the size of $\mathrm{ZnS}$ phosphor microparticles is much higher than that of $\mathrm{BaTiO}_{3}$ nanoparticles, the surface to volume ratio of $\mathrm{BaTiO}_{3}$ nanoparticles in the dielectric film is much higher, leading to a stronger MWS effect. As shown in Figure 6.13(b), the peak $M$ ” 


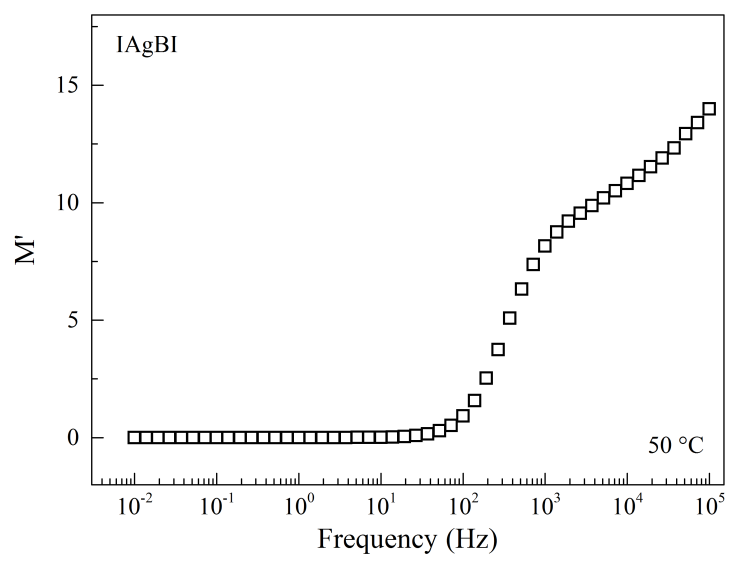

(a)

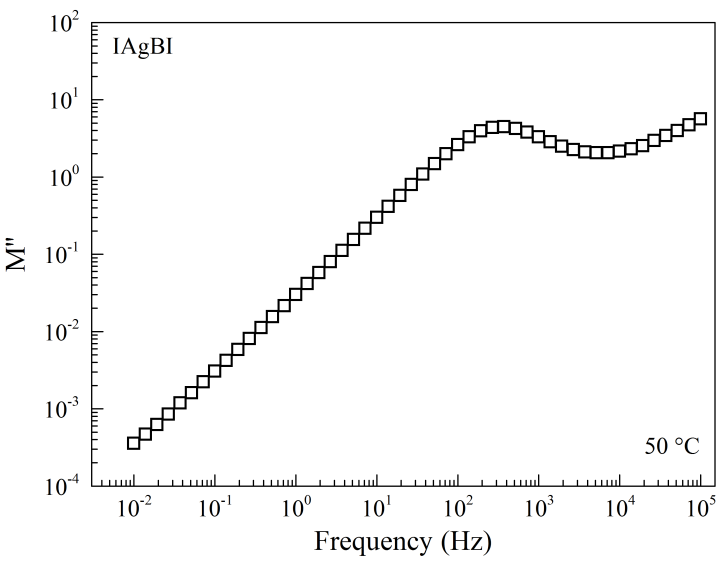

(b)

Figure 6.12: (a) M' and (b) M" as a function frequency at different temperatures for IAgBI.

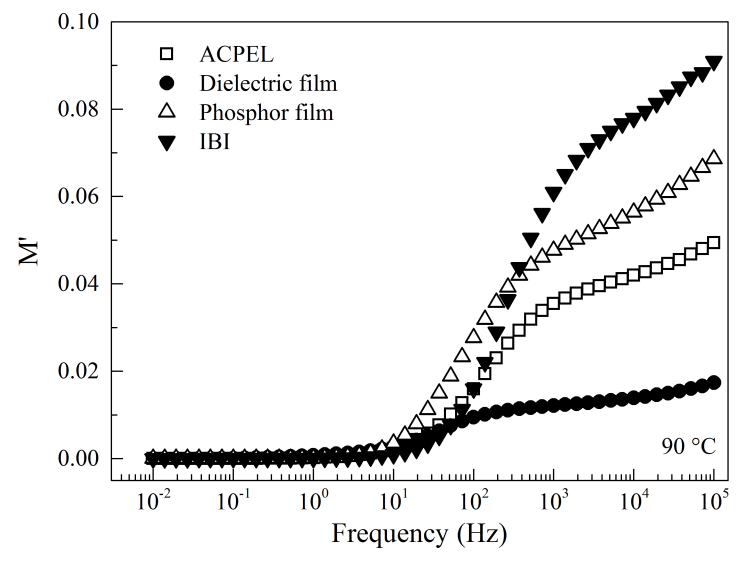

(a)

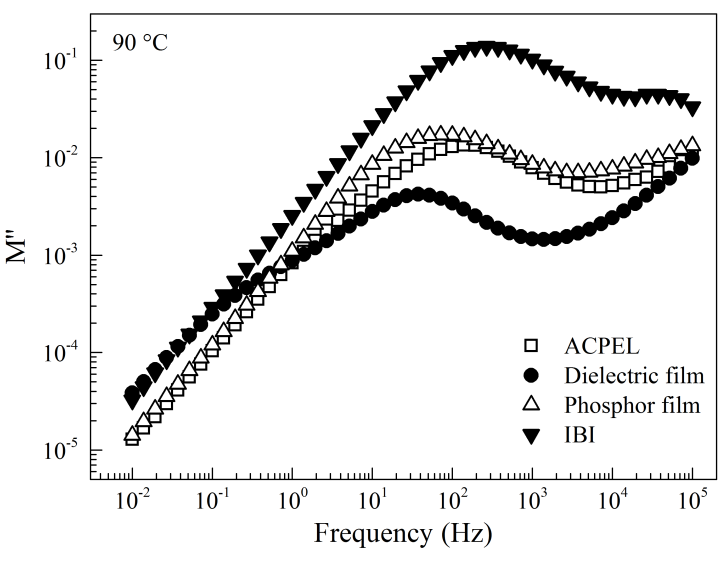

(b)

Figure 6.13: (a) M' and (b) M" as a function frequency at different temperatures for ACPEL devices, dielectric film, phosphor film and IBI. Note: the magnitudes of M' and M' for IBI are scaled by a factor of 0.002 and 0.01 .

value due to the $\alpha$ relaxation in the phosphor film is smaller than that of IBI, which indicates that there is a certain degree of interaction between $\mathrm{ZnS}$ and binder at the $\mathrm{ZnS} /$ binder interfaces. Therefore, the MWS effect may exist at the ZnS/binder interfaces.

In Figures 6.8-6.11, as temperature increases, the $M^{\prime}$ or $M$ ” dispersion shifts to higher frequency. This corresponds to a higher motion of polymer chains at higher temperatures and thus it requires less time to response to the alternating frequency. Similarly, in Figure 6.9(b), the peak frequency related to the MWS effect also shifts to a higher value at 


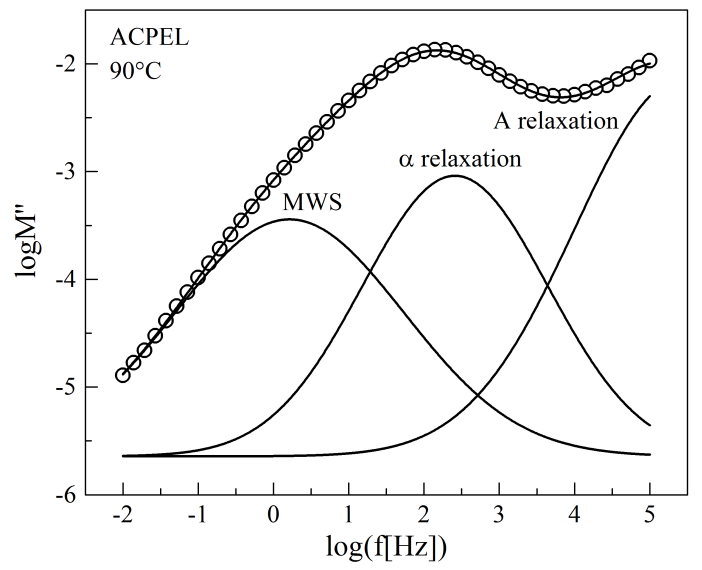

(a)

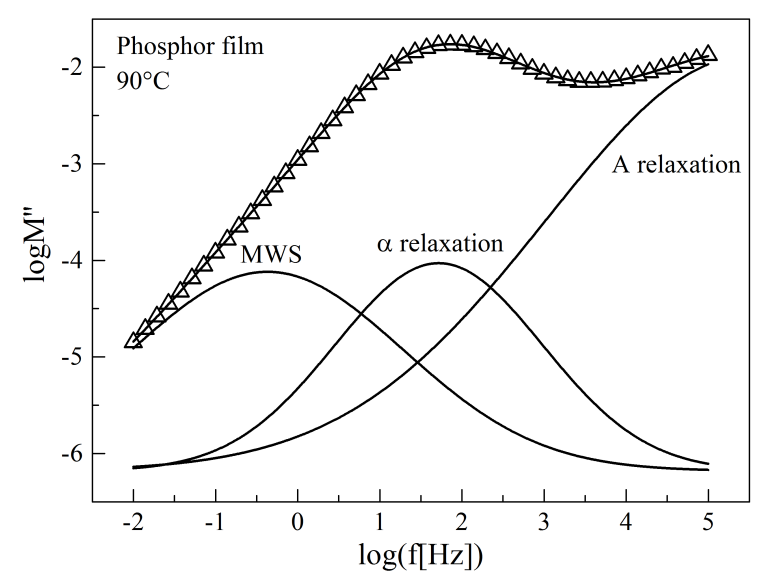

(c)

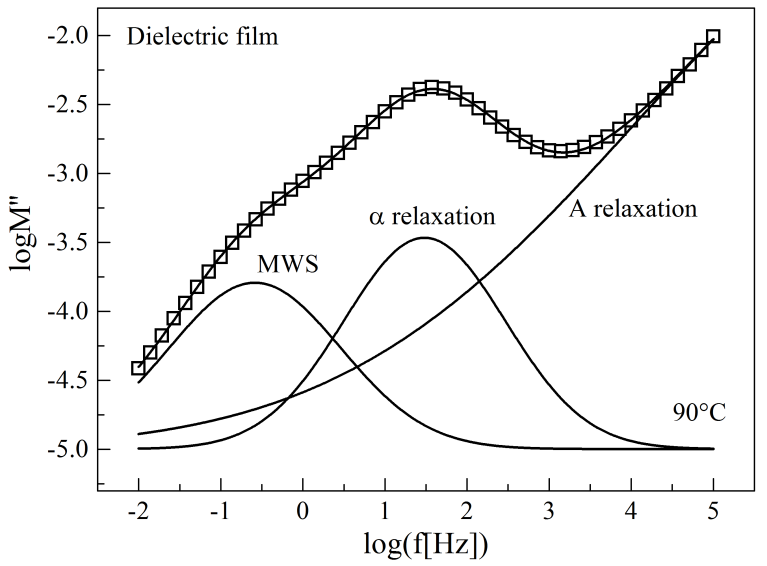

(b)

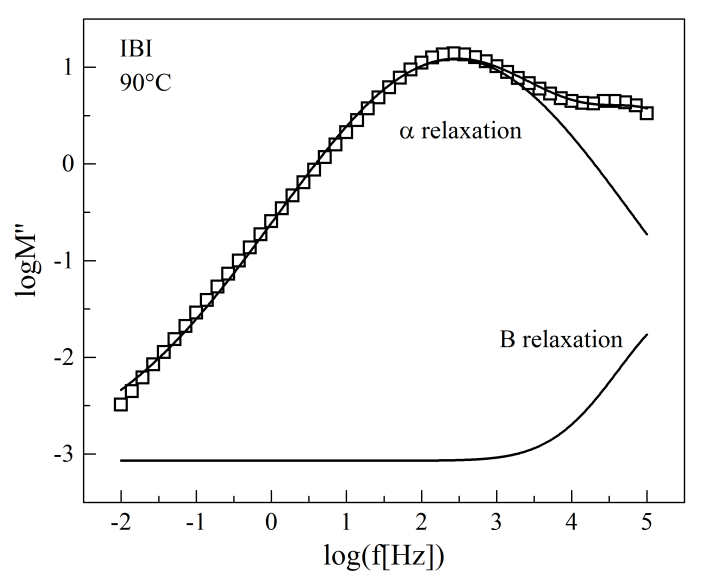

(d)

Figure 6.14: The decomposition of $M$ " dispersion for (a) ACPEL devices, (b) dielectric film, (c) phosphor film, and (d) IBI at $90{ }^{\circ} \mathrm{C}$.

higher temperatures. This is also attributed to the increased mobility of charges at the $\mathrm{BaTiO}_{3} /$ binder interfaces at higher temperatures. As shown in Figure 6.8(b), the $M$ ” dispersion in the ACPEL devices lies between that of the dielectric film and phosphor film, which again proves the superposition theory of ACPEL devices.

In order to analyze the contribution of each relaxation process in various samples, the $M$ " dispersions are fitted using a multi-peak fitting procedure. One of the fittings at 90 ${ }^{\circ} \mathrm{C}$ is shown in Figure 6.14. The $M "-f$ curve of ACPEL devices, dielectric film and phosphor film are well described by the superposition of three Debye-type relaxation processes 


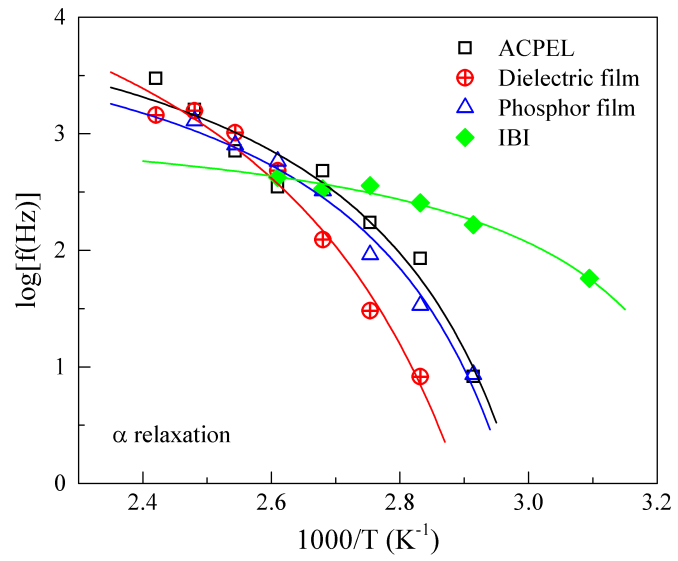

(a)

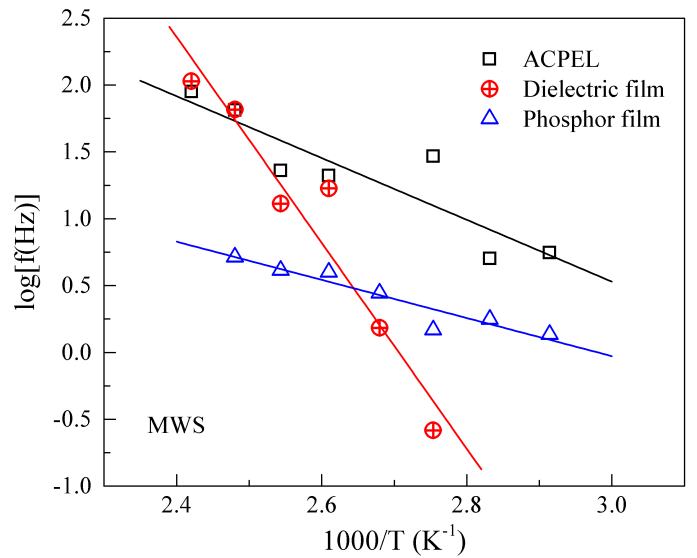

(b)

Figure 6.15: The dependency of (a) $\alpha$ relaxation and (b) MWS effect for ACPEL devices, dielectric film, phosphor film and IBI at elevated temperatures. Solid lines are simulated results.

corresponding to the MWS effect, $\alpha$ relaxation and A/B relaxation from low to high frequencies.

The temperature dependent peak frequency due to $\alpha$ relaxation for ACPEL devices, dielectric film, phosphor film and IBI is shown in Figure 6.15(a). It can be described by the Vogel-Tamann-Fulcher (VTF) equation for all samples according to the following equation [211]:

$$
f_{\max }=f_{0} \exp \left(-\frac{B}{T-T_{0}}\right)
$$

where $f_{0}$ is a pre-exponential factor, $B$ a measure of the activation energy, $T_{0}$ the Vogel temperature or ideal glass transition temperature, and $T$ the temperature in $\mathrm{K}$. The solid lines in Figure 6.15(a) are the well fitted results. The fitted $B$ and $T_{0}$ values for all samples are shown in Table 6.1. $B$, quantifying the activation energy, is the lowest at $115.28 \mathrm{~K}$ in IBI, which means the main chains of binder within IBI are the easiest to experience translational movement. This is straightforward as no fillers are added in the IBI binder and no hinderance is introduced. As the particle size of $\mathrm{BaTiO}_{3}$ is the smallest, the activation energy for the $\alpha$ relaxation in dielectric film is thus the highest. The value of $B$ in phosphor film is $251.10 \mathrm{~K}$, which is larger than that of IBI by a factor of more than 2. This further 
Table 6.1: Activation energy and VTF parameters for ACPEL devivces, dielectric film, phosphor film and IBI.

\begin{tabular}{lccc}
\hline & $\mathrm{E}_{\mathrm{A}}(\mathrm{eV})$ & $\mathrm{B}(\mathrm{K})$ & $\mathrm{T}_{0}(\mathrm{~K})$ \\
\hline ACPEL & 0.46 & 272.02 & 308.59 \\
Dielectric film & 1.53 & 479.79 & 306.06 \\
Phosphor film & 0.28 & 251.10 & 311.02 \\
IBI & & 115.28 & 287.29 \\
\hline
\end{tabular}

proves the existence of interaction between $\mathrm{ZnS}$ microparticles and binder at the $\mathrm{ZnS} / \mathrm{binder}$ interfaces. With the stacking of both $\mathrm{BaTiO}_{3}$ and $\mathrm{ZnS}$ composite layers, ACPEL devices have a $B$ value of $272.02 \mathrm{~K}$, which is between that of the dielectric film and phosphor film. This further proves the superposition theory of ACPEL devices as staking layers of composite films. Moreover, $B$ value of $\alpha$ relaxation in ACPEL is just $20.08 \mathrm{~K}$ higher than that of the phosphor film. This indicates the dominant contribution from the phosphor layer in ACPEL devices. This is due to the lower concentration of $\mathrm{ZnS}$ phosphors and thus higher proportion of binder in the phosphor layer.

The temperature dependency of peak frequency position due to the MWS effect is plotted in Figure 6.15(b), which exhibit an Arrhenius type behaviour in ACPEL devices, dielectric film and phosphor film according to the following equation [113]:

$$
f_{\max }=f_{0} \exp \left(-\frac{E_{\mathrm{A}}}{k_{\mathrm{B}} T}\right)
$$

where $f_{0}$ is a pre-exponential factor, $E_{A}$ the activation energy, and $k_{B}$ the Boltzmann constant. The activation energies of the MWS effect in dielectric and phosphor films are 0.28 and $1.53 \mathrm{eV}$ respectively. This is consistent with the findings by Patsidis and Psarras [145]. With the increase of $\mathrm{BaTiO}_{3}$ concentration, the activation energy of the MWS effect increases. As the concentration of dielectric layer is higher and the size of $\mathrm{BaTiO}_{3}$ nanopar- 
ticles is smaller, there is a higher restriction to the charge movement in response to the alternating electric field. The activation energy of the MWS effect in ACPEL devices is $0.46 \mathrm{eV}$ which is bigger than that of the phosphor film and smaller than that of the dielectric film. This again proves the superposition theory of the MWS effect in ACPEL devices, which includes the contribution from both the $\mathrm{BaTiO}_{3} /$ binder and $\mathrm{ZnS} /$ binder interfaces. Moreover, similar to that of the $\alpha$ relaxation, $\mathrm{E}_{\mathrm{A}}$ of the ACPEL devices is closer to the phosphor film. This is also due to the larger thickness of the phosphor layer.

\subsubsection{Temperature dependent conductivity}

Every material has its conductivity. For materials with high dielectric constant without any DC conductivity, the AC conductivity is caused by the local hopping events of charges within dipoles or at interfaces. The real conductivity $\left(\sigma^{\prime}\right)$ dispersions of ACPEL devices, dielectric film, phosphor film and IBI at elevated temperatures are shown in Figure 6.16. The $\sigma^{\prime}$ dispersion is generally divided into two regions: high frequency non-linear region and low frequency near flat region. The former region is due to the AC conductivity $\left(\sigma_{\mathrm{AC}}\right)$ while the latter one is the DC conductivity $\left(\sigma_{\mathrm{DC}}\right)$. Comparing the $\sigma_{\mathrm{AC}}$ dispersion of IBI in Figure 6.16(d), there are irregularities in the form of several humps in other composite devices especially in dielectric film as shown in Figure 6.16(b). This irregularities are introduced by the interfaces and fillers, which is difficult to be fitted quantitatively. However, the $\sigma_{\mathrm{DC}}$ due to the charge movement within devices follows the VTF equation:

$$
\sigma_{\mathrm{DC}}=\sigma_{0} \exp \left(-\frac{B}{T-T_{0}}\right)
$$

where $\sigma_{0}$ is a pre-exponential factor, $B$ a measure of the activation energy, and $T_{0}$ the Vogel temperature.

The temperature dependency of $\sigma_{\mathrm{DC}}$ in ACPEL devices, dielectric film, phosphor film and IBI is shown in Figure 6.17. The results are all fitted with the VTF equation as indicated 


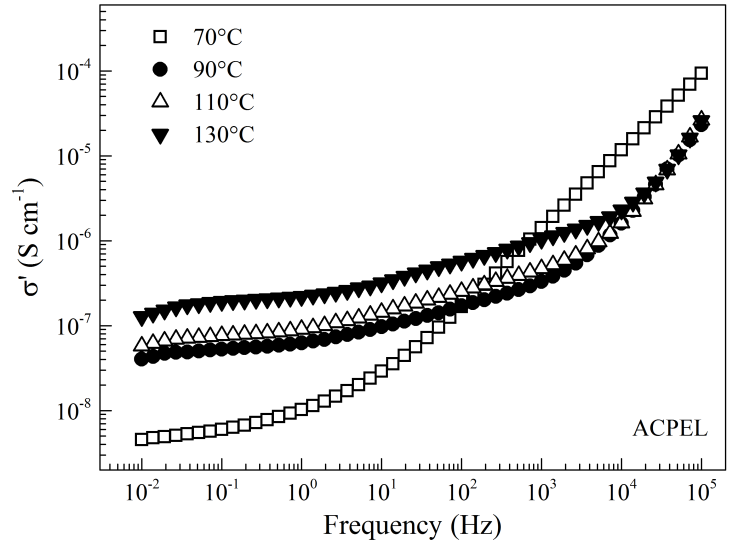

(a)

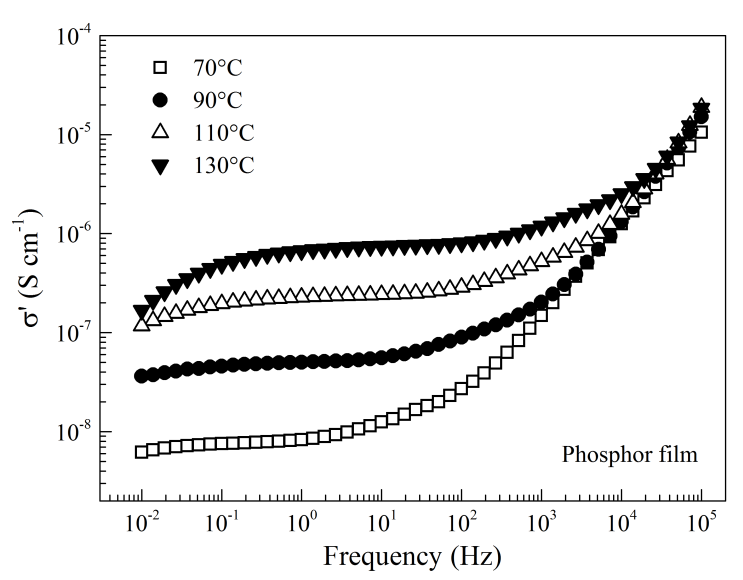

(c)

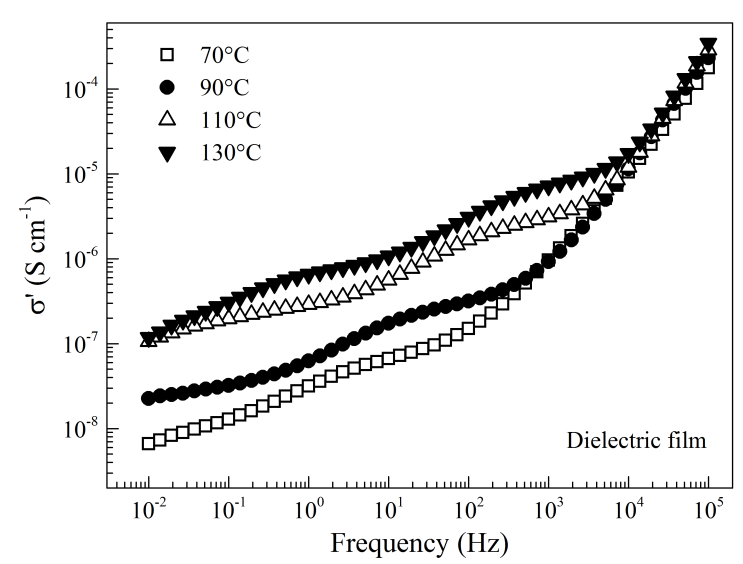

(b)

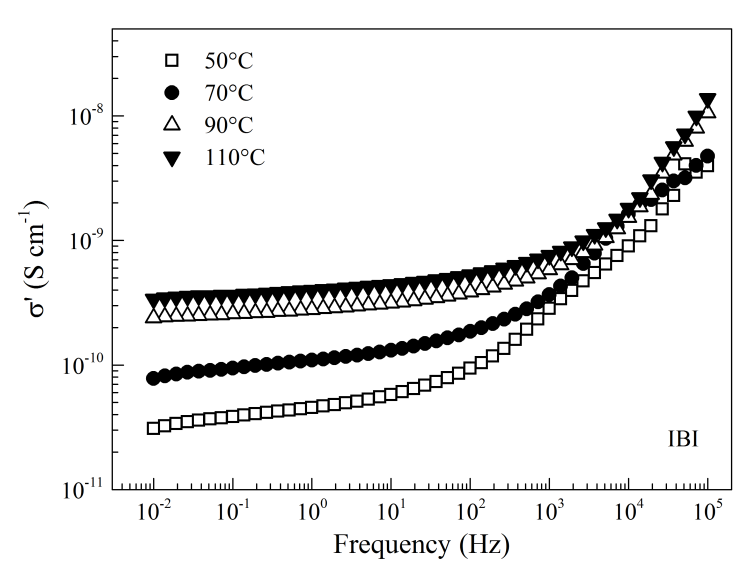

(d)

Figure 6.16: Real conductivity dispersion for (a) ACPEL devices, (b) dielectric film, (c) phosphor film, and (d) IBI at elevated temperatures.

by the solid lines. The fitted $B$ and $T_{0}$ are shown in Table 6.2. IBI has the highest $B$ value of $3546.25 \mathrm{~K}$, which exhibits the strongest resistance to the DC conduction. With $\mathrm{BaTiO}_{3}$ or $\mathrm{ZnS}$ particles added, the activation energy reduces due to the alternate pathway provided by particle/binder interfaces. With the stacking of dielectric and phosphor layers, the activation energy in ACPEL devices is even smaller with the $B$ value of $87.26 \mathrm{~K}$. This shows that alternative pathways may be formed at the interface of dielectric and phosphor layers. 


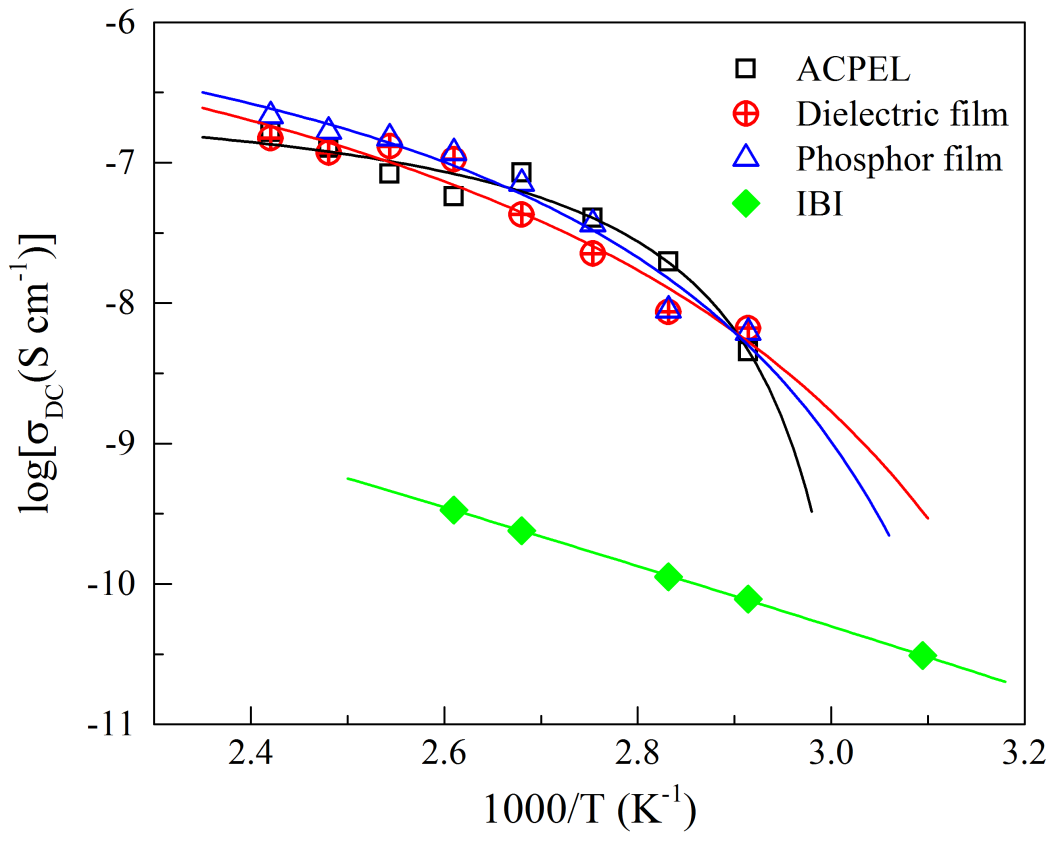

Figure 6.17: The dependency of DC conductivities on temperature in ACPEL devices, dielectric film, phosphor film and IBI . Solid lines are simulated results.

\subsubsection{Equivalent circuit}

From the above analyses, three main relaxation processes caused by different polarization mechanisms are found: EP due to ion accumulation at the electrodes, dipolar reorientation of polymer chains in binder, and the MWS effect at the filler/binder interfaces. The EP takes places in both electrodes with the Ag electrode contributing also to the high frequency "A relaxation". As a result, the dipolar relaxation in binder at intermediate to high frequencies in all the samples may include both the bulk matrix and the Ag electrode. The MWS effect occurs at their respective $\mathrm{ZnS} /$ binder and $\mathrm{BaTiO}_{3} /$ binder interfaces. To better illustrate these relaxation processes, equivalent circuits are constructed for ACPEL devices, dielectric film and phosphor film with the consideration of the superposition effect. Using these equivalent circuits, Nyquist plots are simulated and compared with its experimental impedance data. In Figure 6.18, the fittings between simulated and experimental results are quantified by goodness of fit - chi square values $\chi^{2} \cdot \chi^{2}$ for ACPEL devices, dielectric and phosphor films are $0.001,0.000685$, and 0.006 respectively, which is comparable with the 
Table 6.2: VTF parameters for conductivities in ACPEL devivces, dielectric film, phosphor film and IBI.

\begin{tabular}{lcccc}
\hline & ACPEL & $\begin{array}{c}\text { Dielectric } \\
\text { film }\end{array}$ & $\begin{array}{c}\text { Phosphor } \\
\text { film }\end{array}$ & IBI \\
\hline $\begin{array}{l}\mathrm{B} \\
(\mathrm{K})\end{array}$ & 87.26 & 510.21 & 348.31 & 3546.25 \\
$\mathrm{~T}_{0}$ & 323.10 & 271.32 & & \\
$(\mathrm{~K})$ & & & 291.49 & 52.66 \\
\hline
\end{tabular}

good fittings by Nurk et al. [212]. Therefore, the proposed equivalent circuits is able to well represent the experimental observations.

In Figure 6.18(b), the equivalent circuit for the dielectric film models the relaxation processes contributed by the binder matrix, $\mathrm{BaTiO}_{3}$ nanofillers and electrodes. $R_{s}$ is the series resistance of the external electrical connections. Polarization due to the dipolar reorientation of binder matrix is represented by a resistor $\left(R_{m}\right)$ and capacitor $\left(C_{m}\right)$ in parallel, which describes the conductivity and the dipolar polarization of the binder polymer chains. Another parallel resistor $\left(R_{b t o}\right)$ and capacitor $\left(C_{b t o}\right)$ component is used to model the MWS effect at the $\mathrm{BaTiO}_{3} /$ binder interfaces and also depicts the conductivity and charge accumulation level at the interfaces. The last portion of the equivalent circuit model for the dielectric film describes the double layer nature of the electrode/binder interface. $R_{e}$ is associated with the resistance due to charge transport across the binder matrix close to the electrodes, as suggested by Cha et al.'s physics-based model of polymer-metal composites [151]. $Z_{w}$, the Warburg element, represents the diffusional properties of the mobile ions in the binder matrix towards the electrodes and is quantified by the Warburg admittance $Y_{0 w}$ [213]. $C P E_{e}$ is the constant phase element (CPE) at the electrode/binder interface, which is generally used to represent the imperfect capacitive nature of the space charge layers [214]. This imperfect capacitive phenomenon represents the distribution of time constants at the electrode/binder interfaces. In Figure 6.18(c), the equivalent circuit for the phosphor film 


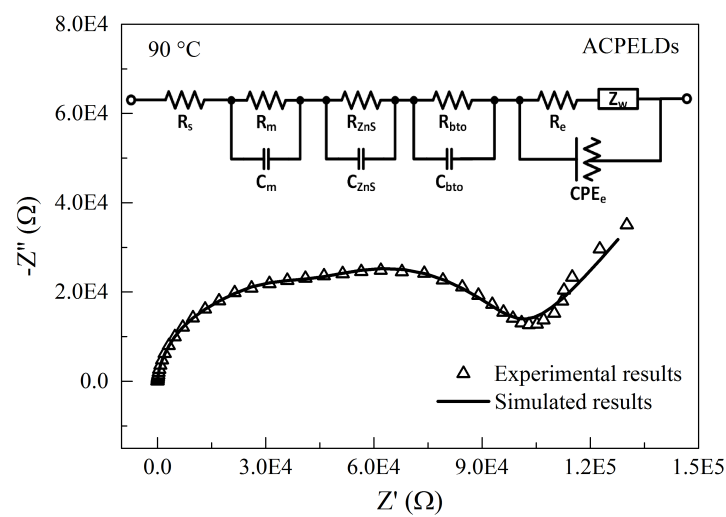

(a)

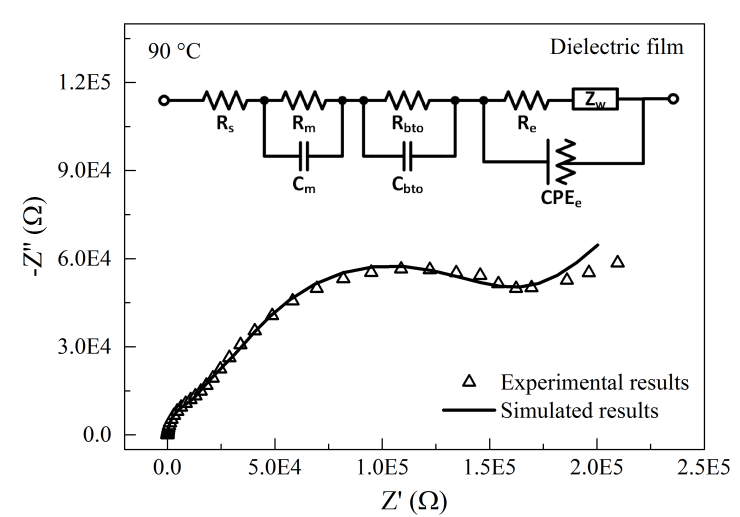

(b)

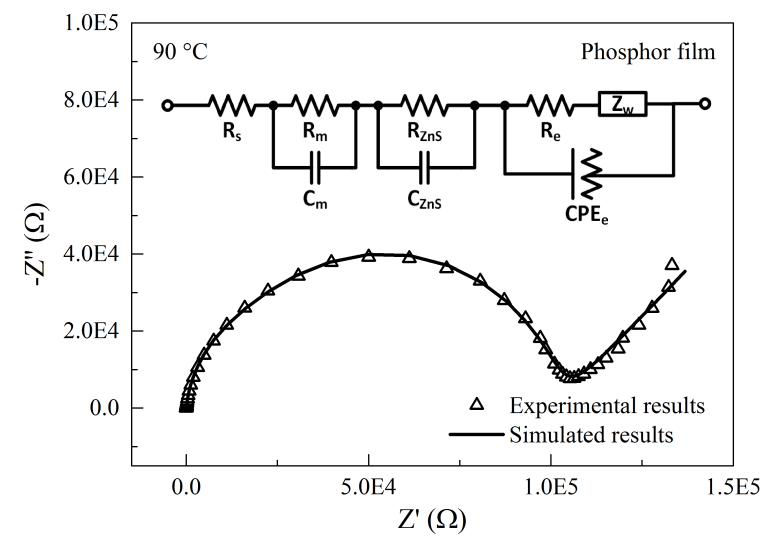

(c)

Figure 6.18: Experimental and simulated Nyquist plots for (a) ACPEL devices, (b) dielectric film, and (c) phosphor film at $90^{\circ}$. Insets: respective equivalent circuits.

is identical to the dielectric film with the exception that the parallel $\mathrm{RC}\left(\mathrm{R}_{\mathrm{ZnS}}\right.$ and $\left.\mathrm{C}_{\mathrm{ZnS}}\right)$ component stands for the MWS effect at the $\mathrm{ZnS} /$ binder interface instead. The equivalent circuit for ACPEL devices is the superposition of the equivalent circuits of the dielectric and phosphor films as shown in Figure 6.18(a).

\subsection{Conclusion}

In conclusion, the relaxation processes of ACPEL devices together with the dielectric film and phosphor film were analyzed by complex impedance spectroscopy from $10^{-2} \mathrm{~Hz}$ to $10^{5}$ $\mathrm{Hz}$ at elevated temperatures. Three types of polarization mechanisms were identified in 
these three samples: electrode polarization due to ion accumulation at the electrode/binder interfaces, the MWS effect at the $\mathrm{ZnS} /$ binder and $\mathrm{BaTiO}_{3} /$ binder interfaces and polarization due to the dipolar reorientation of polymer chains in the binder matrix. The MWS effect at the $\mathrm{BaTiO}_{3} /$ binder interface is much more significant than the $\mathrm{ZnS} /$ binder interface due to the higher surface-volume ratio by smaller $\mathrm{BaTiO}_{3}$ nanoparticles. On the other hand, dipolar polarization of binder in the phosphor film is more significant than the dielectric film due to the larger volume fraction of binder in the phosphor layer. The dipolar polarization of binder includes the contribution from both the bulk and Ag electrode. The DC conductivity of these devices follows VTF equation. Finally, these polarization mechanisms are modeled using electrical circuit components and equivalent circuit models for these devices were derived. The simulated circuit model, which shows good fitting with its experimental results, provides a better understanding of the ACPEL device architecture and enabled an effective non-destructive diagnosis of ACPEL devices and other printed electronics applications. 


\section{Chapter 7}

\section{OPTO-IMPEDANCE SPECTROSCOPY OF ACPEL DEVICES}

\subsection{Introduction}

As presented in Chapter 6, the impedance spectroscopy study of ACPEL devices revealed the existence of three main relaxation processes: $\mathrm{EP}, \alpha$ relaxation, and the MWS effect at filler/binder interfaces. Corresponded equivalent circuit model was also proposed to represent these relaxation processes. However, this equivalent circuit model of the unexcited ACPEL device may not apply to the excited ones under high electric field. This is due to the involvement of hot electrons and their interaction with dielectrics and metal contacts [215]. The conventional impedance spectroscopy methodology only utilizes a low strength signal. Alternatively, $\mathrm{ZnS}$ phosphor can be photo-excited and it has been proven that photoexcited ACPEL devices exhibit similar dielectric response to the equivalent electrically excited ones [49]. Roberts found that $\mathrm{ZnS}_{0.8} \mathrm{Se}_{0.2}: \mathrm{Cu}$ phosphors were sensitive to high energy photons (365 nm to $436 \mathrm{~nm}$ ) and the dielectric constant of their EL films increased with illumination intensity [49]. The illumination intensity caused a shift of the dielectric constant in the same fashion as the change in the excitation voltage. On the other hand, Soudek observed that the resistance of $\mathrm{ZnCdS}$ :Ag phosphors was reduced under short-wavelength illuminations and attributed it to the quenching of luminescence and conductivity [216]. Kronenberg and Accardo studied the impedance changes of $\mathrm{ZnS} / \mathrm{CdS}$ phosphor particles under different illumination intensities and found that the resistance decreased while the capacitance increased with intensity [217]. ACPEL devices, comprising of stacked layers of polymer composites containing both micro- and nanofillers, are susceptible to dielectric dispersions due to the dielectric relaxation of polymer matrix, fillers and filler/matrix interfaces [218]. The impedance changes on these photo-sensitive phosphor particles can 
cause dielectric dispersion within the ACPEL structure and requires a different excitation voltage/frequency condition to compensate for the required luminescence output. Existing simplified equivalent circuit models find it difficult to derive the equivalent circuit according to the device architecture due to its high impedance at room temperature. Furthermore, complexity is added if the photo-sensitivity of phosphors is taken into account [34, 205].

In this chapter, the effects of illumination wavelengths and intensities on the dielectric dispersion of ACPEL devices were studied, which is also known as opto-impedance spectroscopy. To identify the material sensitive to high-energy photons and study the optoimpedance response in ACPEL devices, the dielectric properties of phosphor ( $\mathrm{ZnS}$ microparticles) and dielectric $\left(\mathrm{BaTiO}_{3}\right.$ nanoparticles) films were also analyzed. Equivalent circuit models for the ACPEL devices and phosphor films were then constructed and compared.

\subsection{Experimental methods}

\subsubsection{Experiment setup}

Three types of samples were fabricated using screen printing process: ACPEL devices, a dielectric film and a phosphor film. The device structure and fabricating process for these three samples can be referred to Chapter 6. The study of opto-impedance of ACPEL device was conducted with a customized setup according to Figure 7.1. It consisted of an enclosure with a RGB LED panel and a Peltier-based heater inside, a DC power supply, a temperature controller and a frequency impedance analyzer. The enclosure shielded the DUT from EMI and external illumination from other sources. The DC power supply Keithley 2636A was used to adjust the light intensity level of the LED panel, whose optical properties were measured using a halfmoon integrating sphere from Labsphere. The peak wavelengths from the measurements showed $399 \mathrm{~nm}(\mathrm{FWHM} \sim 12 \mathrm{~nm}), 520 \mathrm{~nm}(\mathrm{FWHM} \sim 30 \mathrm{~nm})$ and $625 \mathrm{~nm}$ (FWHM $\sim 11 \mathrm{~nm}$ ) accordingly. To ensure a uniform illumination on the test specimen, the LED panel was placed $20 \mathrm{~cm}$ above the DUT. The spatial illumination resolution on the 


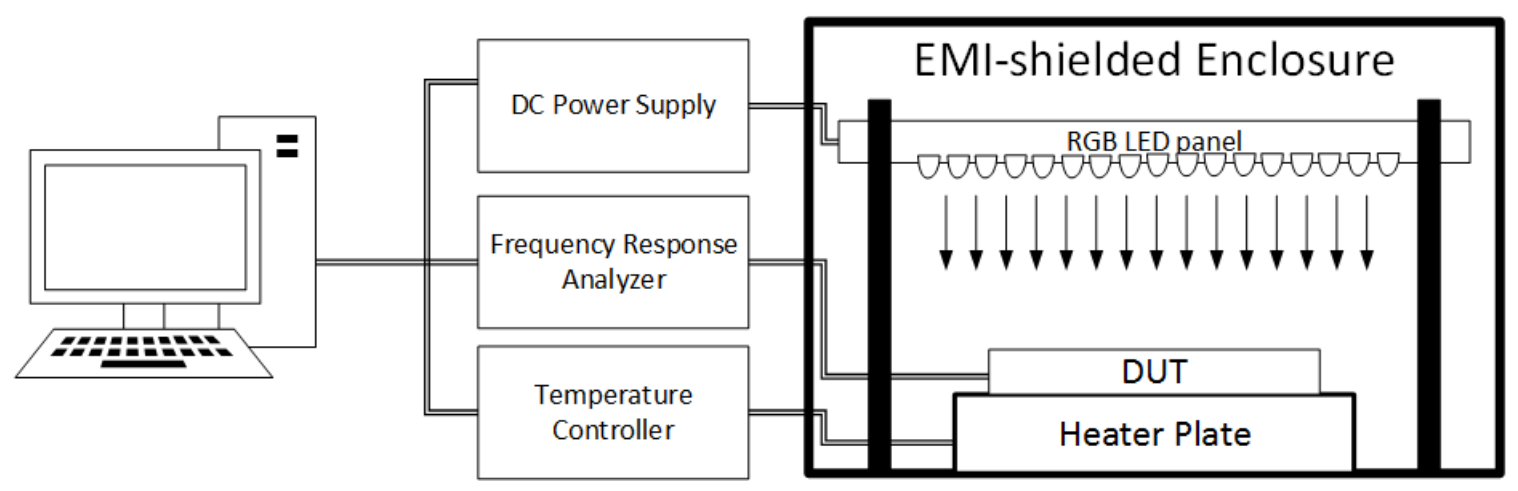

Figure 7.1: Experiment setup for opto-impedance measurement and analysis of the ACPEL devices, phosphor and dielectric films.

DUT was less than 5\% as revealed by luminance measurements by the PR655 spectroradiometer from Photo Research. The DUT was adhered onto the Peltier-based heater plate via a thermal pad and was controlled by a Accuthermo ATEC302 temperature controller. Its spatial temperature resolution was $+/-0.2{ }^{\circ} \mathrm{C}$. The opto-impedance data was obtained using Autolab PGSTAT302N with samples heated to $90{ }^{\circ} \mathrm{C}$. The impedance data was obtained using Autolab PGSTAT302N equipped with a frequency response analyser FRA2 module, which has a current range from $10 \mathrm{nA}$ to $10 \mathrm{~mA}$ with resolution of $0.0003 \%$ and a potential resolution of $3 \mathrm{~V}$. The dielectric data was measured by supplying an unbiased voltage signal with a peak voltage of $0.5 \mathrm{~V}$ and a frequency range of $10^{-2}$ to $10^{5} \mathrm{~Hz}$ with a resolution of $0.0003 \%$. The $\varepsilon^{\prime}, \varepsilon^{\prime \prime}, M$ " and $\sigma^{\prime}$ were calculated according to Equations 2.42-2.47. The equivalent circuits were simulated by both Nova and Zview software. The PL emission, excitation and phosphorescence lifetime were measured by Fluorolog-3 PL system.

\subsubsection{Experiment procedure}

All the measurements were taken at $90{ }^{\circ} \mathrm{C}$, which is a representative temperature as proposed in Chapter 6. All the three types of samples went through the same measurements. Firstly, the DUT was loaded onto the heater. With temperature stabilized at $90{ }^{\circ} \mathrm{C}$, the impedance data was measured from $10^{-2}$ to $10^{5} \mathrm{~Hz}$. The measurement took about $15 \mathrm{~min}$. 
After the measure was finished, a $625 \mathrm{~nm}$ illumination with the exciting current of $40 \mathrm{~mA}$ was applied over the DUT by the LED panel. When the temperature was stabilized at 90 ${ }^{\circ} \mathrm{C}$, the impedance measurement was carried out. This repeats for exciting currents of 40 , 100, 400 and $800 \mathrm{~mA}$. A similar procedure was followed for the $520 \mathrm{~nm}$ and $399 \mathrm{~nm}$ illuminations. The equivalent radiative power intensities of the illumination excited by these currents were measured using the halfmoon integrating sphere.

\subsection{Results and discussion}

\subsubsection{Wavelength effects}

\section{Relative permittivity}

The relative permittivity $\varepsilon^{*}$ dispersions of ACPEL devices, dielectric film and phosphor film are shown in Figures 7.2-7.4. It is seen that the $520 \mathrm{~nm}$ and $625 \mathrm{~nm}$ photons do not cause any significant changes to both $\varepsilon^{\prime}$ and $\varepsilon^{\prime \prime}$ for all three devices. This shows no optoimpedance behavior by these two wavelengths. On the contrary, the $399 \mathrm{~nm}$ illumination affects both ACPEL devices and the phosphor film but not the dielectric film, either for $\varepsilon$ ' or $\varepsilon "$. This suggests that the opto-impedance behvavior does not take place in the dielectric layer but rather in the ZnS phosphor layer. In Figures 7.2(a) and 7.4(a), the $\varepsilon$ ' dispersions of ACPEL devices and phosphor film are shifted upward due to the $399 \mathrm{~nm}$ illumination. This shows that the polarization of these two devices is increased when photo-excited by the $399 \mathrm{~nm}$ photons. In Figures 7.2(b) and 7.4(b), $\varepsilon$ " dispersions of the ACPEL devices and phosphor film are also shifted upward to higher values indicating that higher energy loss is induced by the $399 \mathrm{~nm}$ illumination.

\section{Electric modulus}

As discussed in Chapter 6, electric modulus $M^{*}$ plots effectively suppress the DC conduction within ACPEL devices. Thus it gives the true relaxation process in the bulk material. 


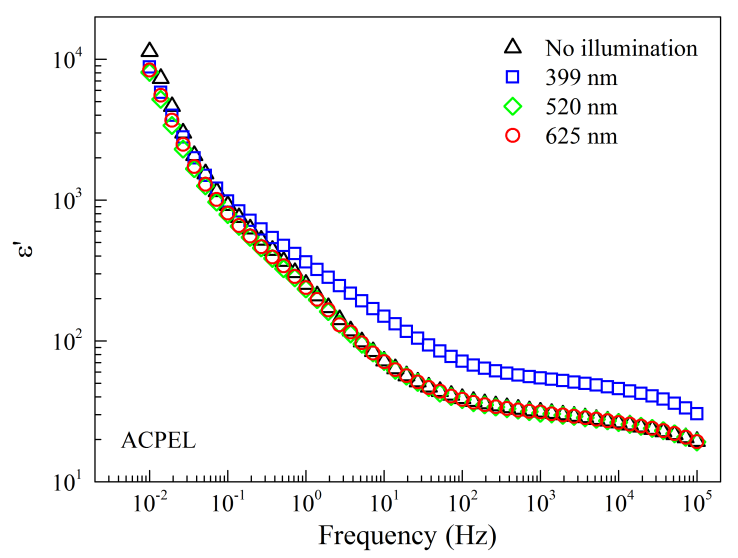

(a)

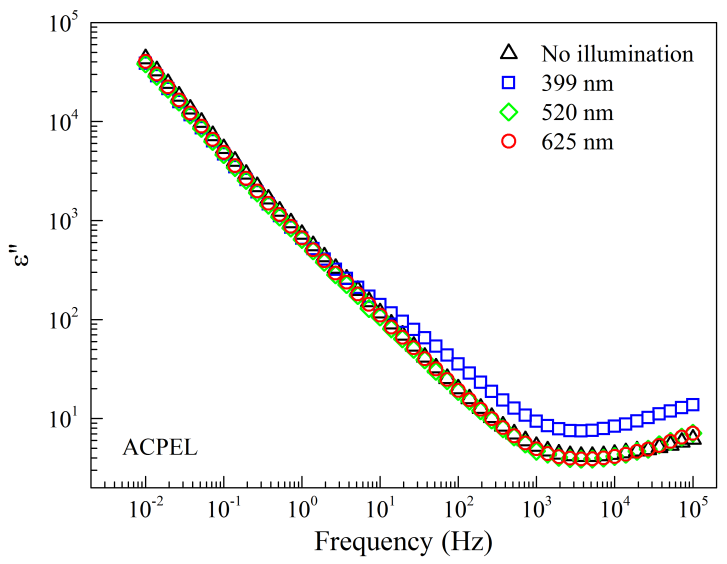

(b)

Figure 7.2: (a) $\varepsilon$ ' and (b) $\varepsilon$ " as a function of frequency without illumination and with 399 $\mathrm{nm}, 520 \mathrm{~nm}$ and $625 \mathrm{~nm}$ illuminations for ACPEL devices.

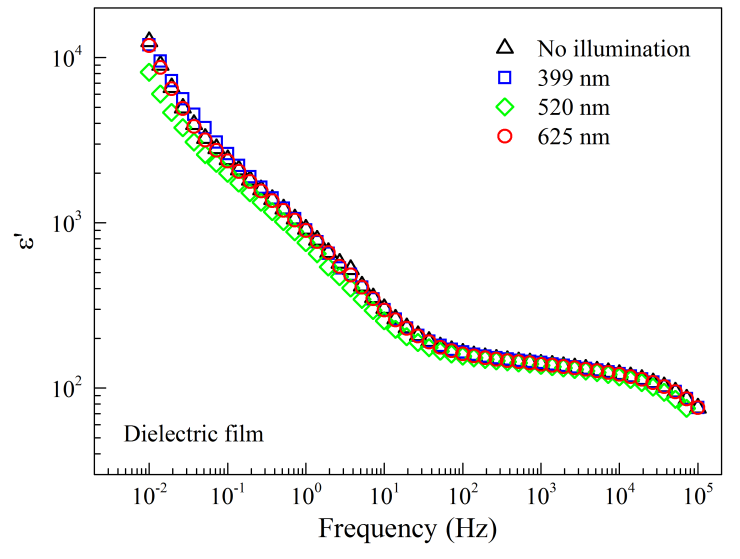

(a)

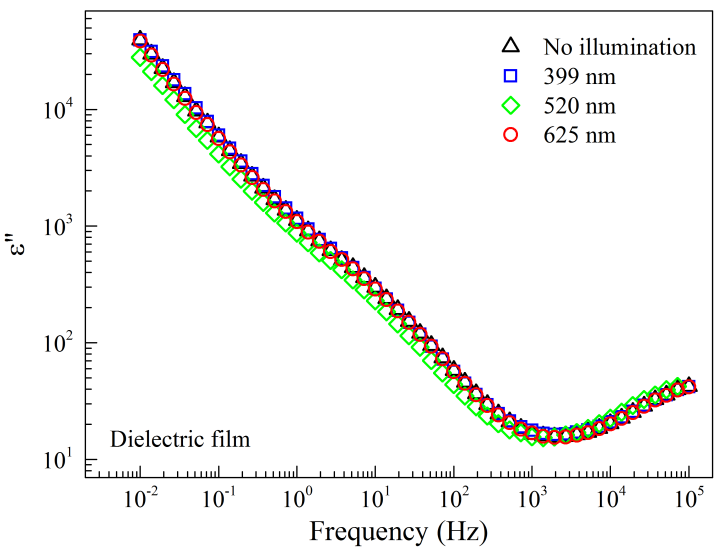

(b)

Figure 7.3: (a) (a) $\varepsilon$ ' and (b) $\varepsilon$ " as a function of frequency without illumination and with $399 \mathrm{~nm}, 520 \mathrm{~nm}$ and $625 \mathrm{~nm}$ illuminations for the dielectric film.

In Figures 7.5-7.7, the $520 \mathrm{~nm}$ and $625 \mathrm{~nm}$ illuminations do not bring any change to the $M^{\prime}$ and $M$ " dispersions in any of these three devices. Moreover, the electric modulus of the dielectric film is not affected by any of these three illumination wavelengths. This further indicates that the relaxation processes in the $\mathrm{BaTiO}_{3}$ nanocomposites are not affected by illumination directly. The $399 \mathrm{~nm}$ illumination causes both $\mathrm{M}^{\prime}$ and $M$ " to decrease in both ACPEL devices and phosphor film, indicating that the relaxation processes within the phosphor layer are directly affected by the high energy photons. As studied in Chapter 6, the 


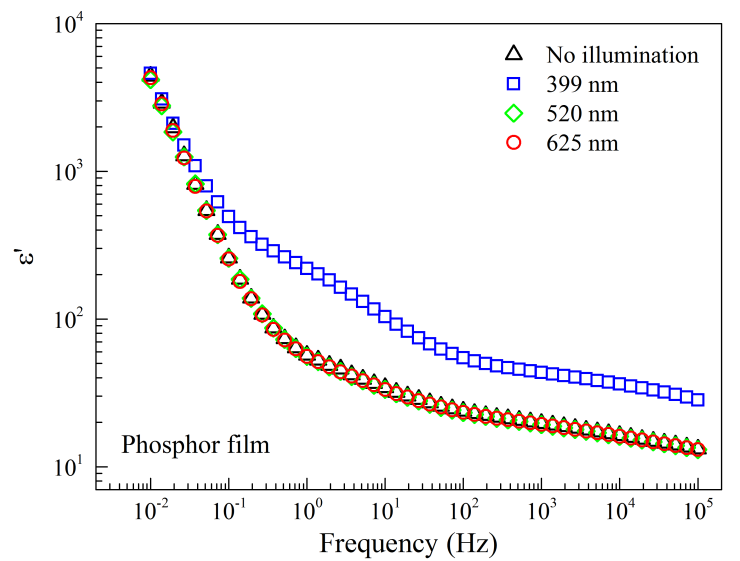

(a)

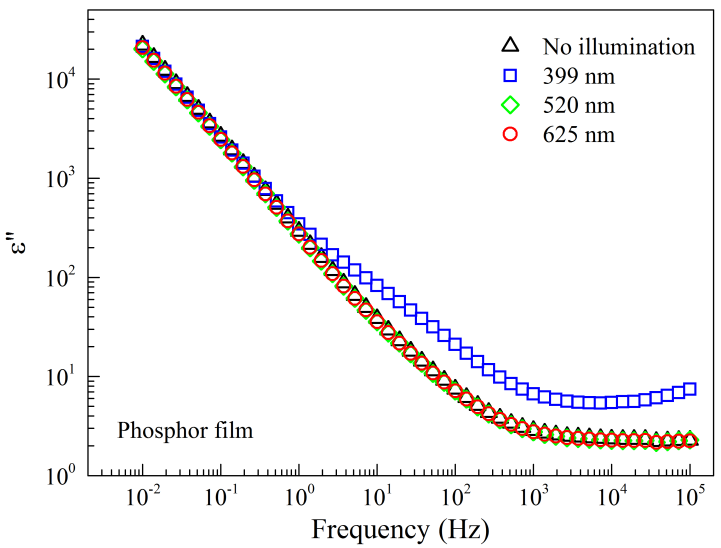

(b)

Figure 7.4: (a) $\varepsilon$ ' and (b) $\varepsilon$ " as a function of frequency without illumination and with 399 $\mathrm{nm}, 520 \mathrm{~nm}$ and $625 \mathrm{~nm}$ illuminations for the phosphor film.

relaxation processes existing in the phosphor layer are the MWS effect at the $\mathrm{ZnS} / \mathrm{binder}$ interfaces and the $\alpha$ relaxation. As labelled in Figures 7.5(b) and 7.7(b), the $M$ " peak due to the overall effect of the MWS effect and $\alpha$ relaxation is also shifted to higher frequency. This corresponds to the smaller time constants and faster response caused by the $399 \mathrm{~nm}$ illumination.

\section{Conductivity}

The $\sigma^{\prime}$ dispersions in ACPEL devices, dielectric film and phosphor film without illumination and with $399 \mathrm{~nm}, 520 \mathrm{~nm}$ and $625 \mathrm{~nm}$ illuminations are shown in Figure 7.8. Similar to the results of relative permittivity and electric modulus in previous two sections, the $520 \mathrm{~nm}$ and $625 \mathrm{~nm}$ illuminations do not affect the $\sigma^{\prime}$ at all frequencies. Only the 399 $\mathrm{nm}$ illumination shifts the $\sigma^{\prime}$ dispersions of ACPEL devices and phosphor film upward. The conductivity of the dielectric film is not affected by any of the three illuminations. As shown in Figures 7.8(a) and 7.8(c), the opto-impedance effect introduced by the $399 \mathrm{~nm}$ illumination increases the conductivity at high frequency but not low frequency. As the low frequency conductivity is the DC conductivity, thus the mobility of the ionic carrier contributing to DC conduction is not altered by the illumination. Only those charge carriers 


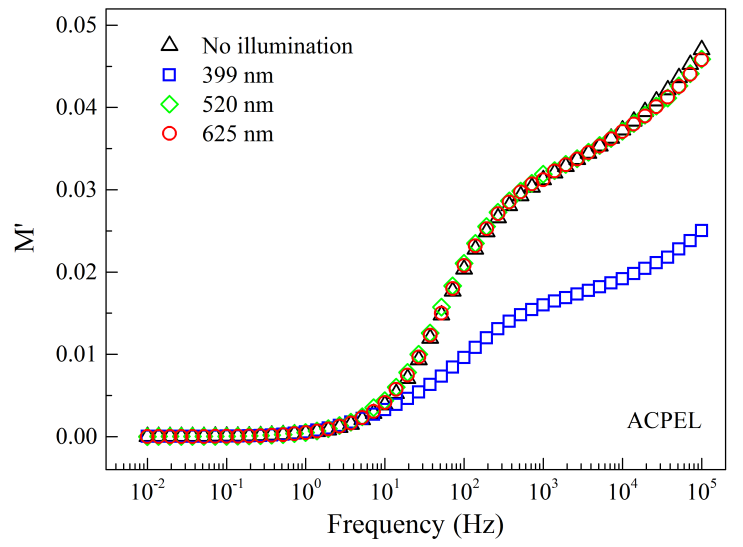

(a)

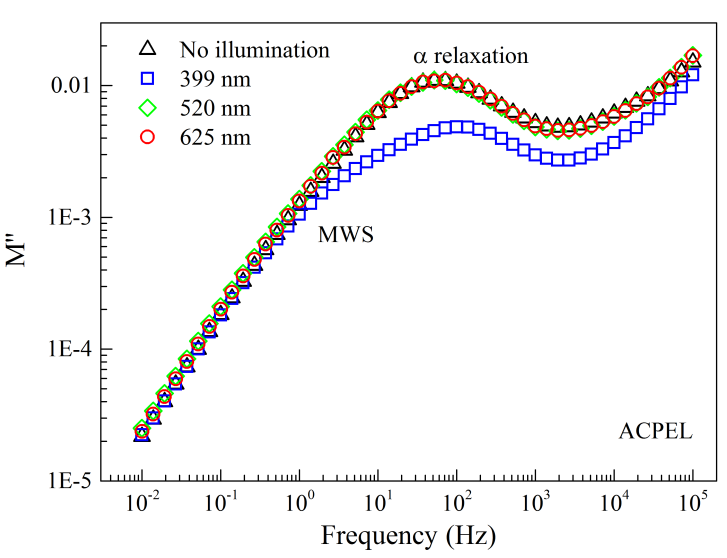

(b)

Figure 7.5: (a) $M$ ' and (b) $M$ ' as a function of frequency without illumination and with 399 $\mathrm{nm}, 520 \mathrm{~nm}$ and $625 \mathrm{~nm}$ illuminations for ACPEL devices.

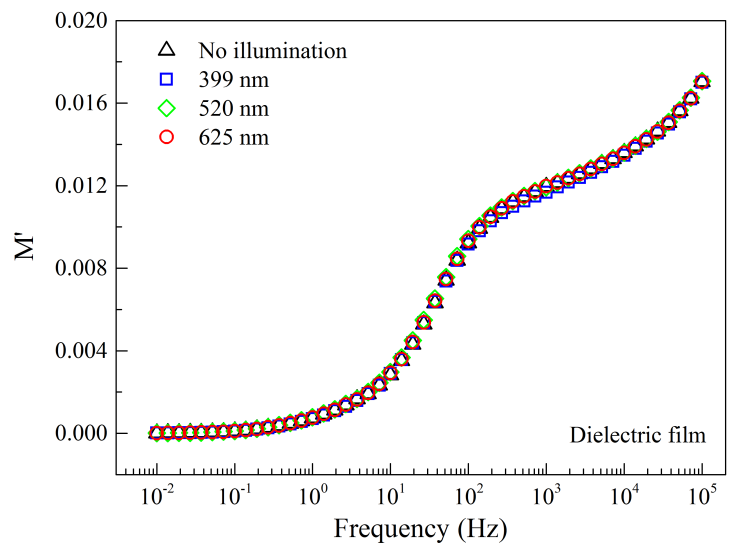

(a)

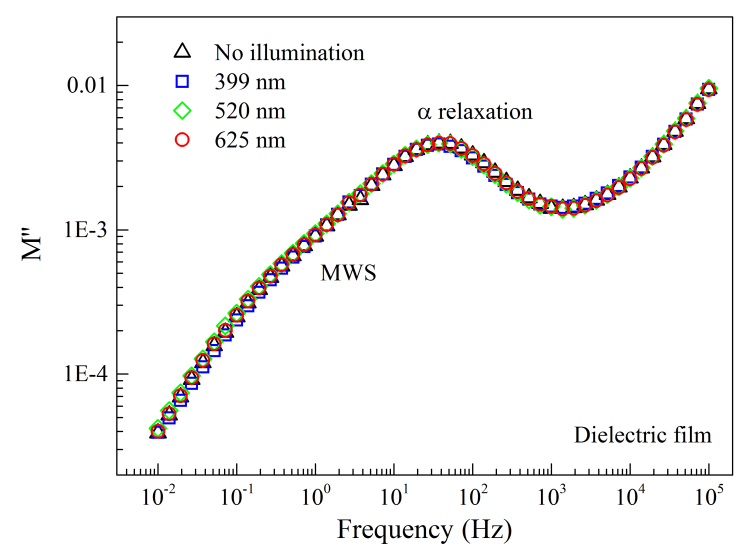

(b)

Figure 7.6: (a) (a) $M^{\prime}$ and (b) $M$ " as a function of frequency without illumination and with $399 \mathrm{~nm}, 520 \mathrm{~nm}$ and $625 \mathrm{~nm}$ illuminations for the dielectric film.

associated with the interfaces and bulk matrix are directly or indirectly influenced by the high energy photons.

\section{Mechanism for the wavelength effects}

This aforementioned wavelength-dependent opto-impedance behavior is ascribed to the excitation of $\mathrm{ZnS}$ phosphors by PL which is further affirmed by PL emission, excitation and absorbance measurements. $\mathrm{BaTiO}_{3}$ nanoparticles used in this study have a large bandgap 


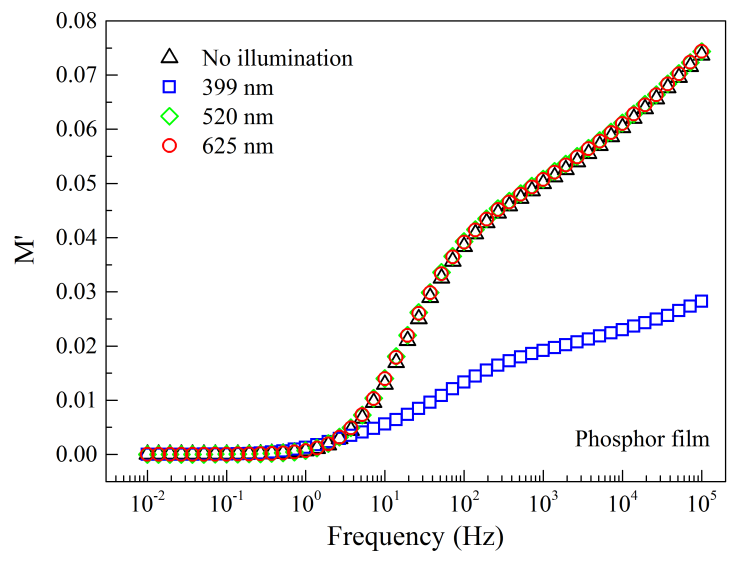

(a)

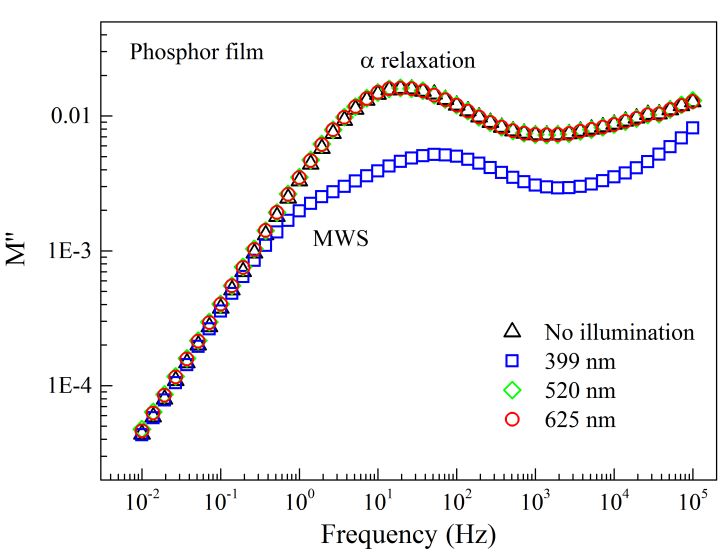

(b)

Figure 7.7: (a) $M^{\prime}$ and (b) $M$ "' as a function of frequency without illumination and with 399 $\mathrm{nm}, 520 \mathrm{~nm}$ and $625 \mathrm{~nm}$ illuminations for the phosphor film.

and do not absorb photons from $300 \mathrm{~nm}$ to $900 \mathrm{~nm}$ through the absorbance measurement by UV-Vis. As shown in Figure 7.9, $\mathrm{ZnS}$ :Cu,Al phosphors with the peak wavelength of 500 $\mathrm{nm}$ can be excited by incident photons with wavelengths below $420 \mathrm{~nm}$. The wavelength spectra of the $520 \mathrm{~nm}$ and $625 \mathrm{~nm}$ illuminating sources do not overlap with the excitation spectrum of $\mathrm{ZnS}$ phosphors and only the $399 \mathrm{~nm}$ illumination has sufficient energy to excite $\mathrm{ZnS}$ phosphors in the ACPEL devices and phosphor films. Based on the PL lifetime measurement as shown in the inset of Figure 7.9, these $\mathrm{ZnS}$ phosphors exhibit phosphorescence with the lifetime of tens of microseconds which matches the results shown by Chen et al. [219]. This long afterglow is believed to be due to the detrapping of trapped electrons in sulphur vacancies [220]. The emission centres of $\mathrm{ZnS}$ phosphor particles were found to be located both in the bulk [79] as well as on its surface [55]. Thus, when ZnS phosphors are excited by high-energy photons, photo-induced charges may be trapped not only in the sulphur vacancies but also in interfaces or other defect sites, leading to the change of dielectric dispersion. 


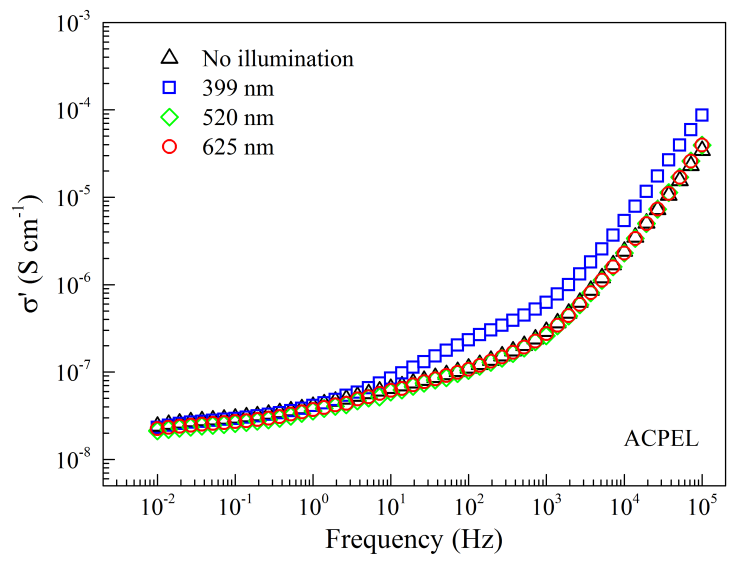

(a)

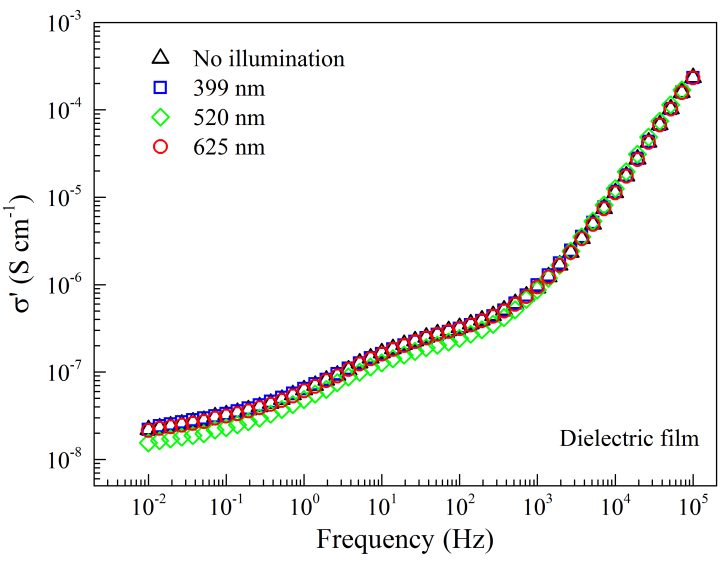

(b)

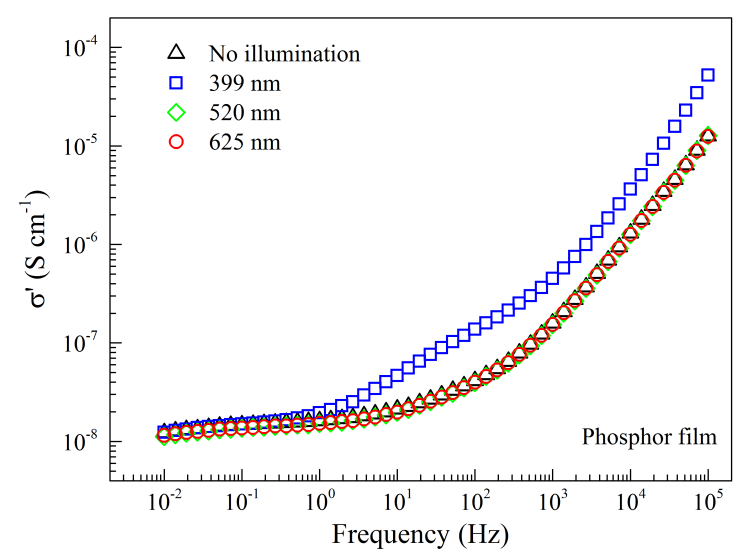

(c)

Figure 7.8: $\sigma^{\prime}$ as a function of frequency without illumination and with $399 \mathrm{~nm}, 520 \mathrm{~nm}$ and $625 \mathrm{~nm}$ illuminations for (a) ACPEL devices, (b) dielectric film and (c) phosphor film.

\subsubsection{Intensity effects}

\section{Relative permittivity}

In order to study the intensity effects, $0.5,1.3,4.9$ and $9.0 \mathrm{mWatts} / \mathrm{cm}^{2}$ of the $399 \mathrm{~nm}$ illumination were applied over ACPEL devices and the phosphor film. The relative permittivity dispersions of these two devices are shown in Figures 7.10 and 7.11. At intermediate to high frequencies, both $\varepsilon$ ' and $\varepsilon$ " increases with illumination intensity and tend to reach a steady state for ACPEL devices and phosphor film. This indicates that the free charges within $\mathrm{ZnS}$ phosphors are also increased by the increased illumination intensity and thus 


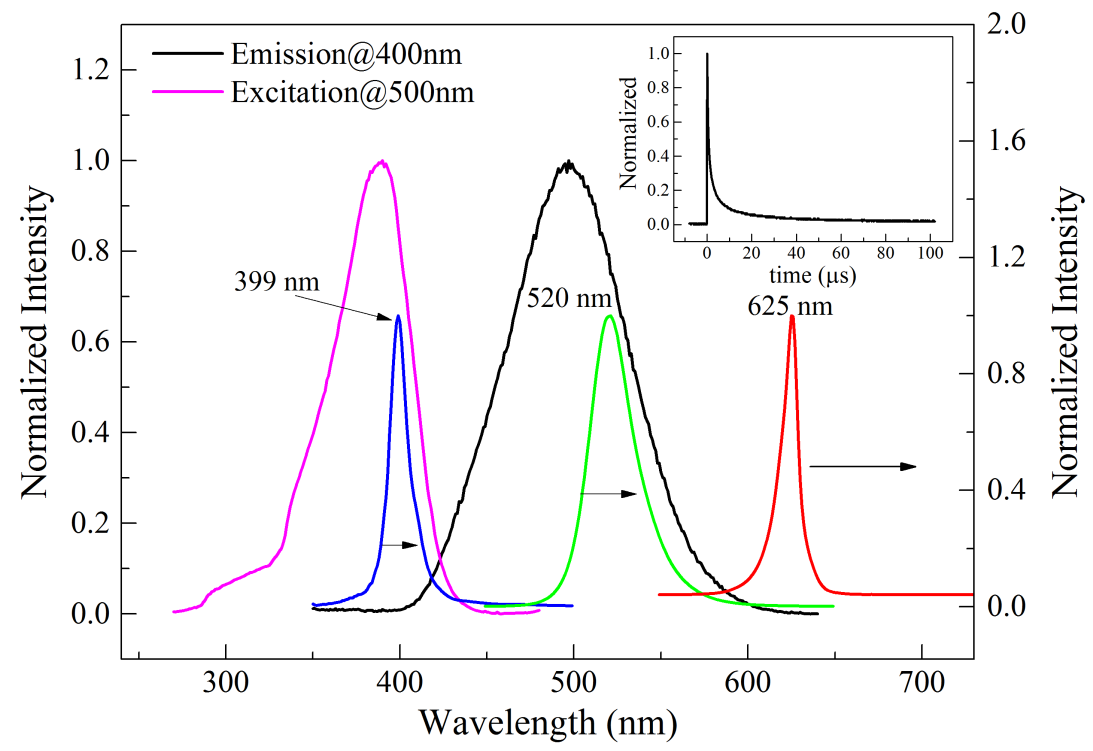

Figure 7.9: Normalized PL emission and excitation spectra of green $\mathrm{ZnS}: \mathrm{Cu}, \mathrm{Al}$ phosphors together with the EL spectra of $399 \mathrm{~nm}, 520 \mathrm{~nm}$ and $625 \mathrm{~nm}$ illuminations. Inset: PL intensity as a function of time after the excitation source is switched off.

the polarization and dielectric loss are increased due to the increased interactions of free charges with the surrounding materials. However, at low frequencies, The linear portions of both $\varepsilon$ ' and $\varepsilon$ " stay unchanged with intensity, which are related to the EP process and DC conductivity.

\section{Electric modulus}

As shown in Figures 7.12 and 7.13, as the $399 \mathrm{~nm}$ illumination is intensified, both $M^{\prime}$ ' and $M$ ” dispersions shift downwards in ACPEL devices and phosphor film. No additional peaks or humps are observed with the increase of intensity in Figures 7.12(b) and 7.13(b). This means that with the increase of the illumination intensity, no additional relaxation process is introduced. The phosphor film only has a $\alpha$ relaxation process in binder and another MWS effect at the ZnS/binder interfaces. As for the ACPEL devices, there are $\alpha$ relaxation and MWS effect at the $\mathrm{BaTiO}_{3} /$ binder and $\mathrm{ZnS} /$ binder interfaces at all intensities. The peak position of $M$ " in both devices shifts toward higher frequency with the increased intensity, 


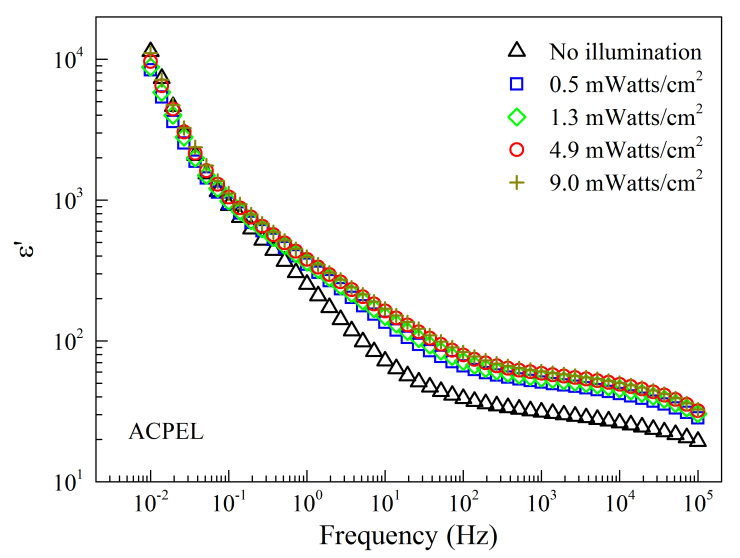

(a)

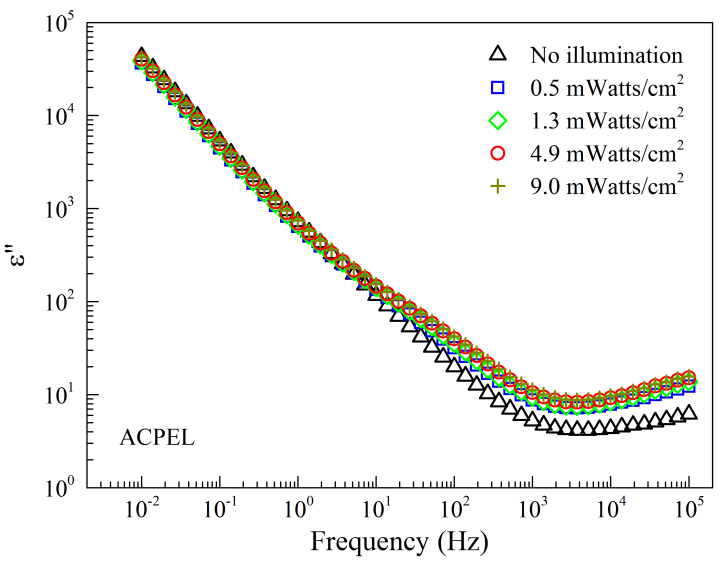

(b)

Figure 7.10: (a) $\varepsilon$ ' and (b) $\varepsilon$ " as a function of frequency with the $399 \mathrm{~nm}$ illumination at different intensities for ACPEL devices.

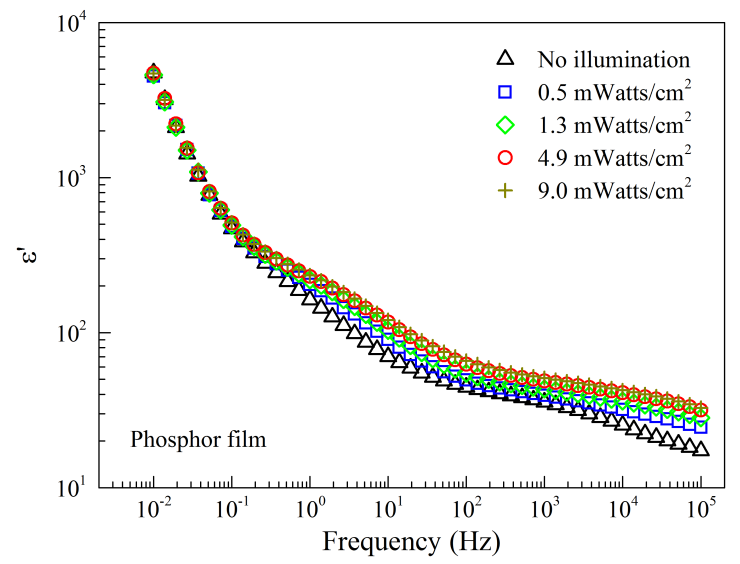

(a)

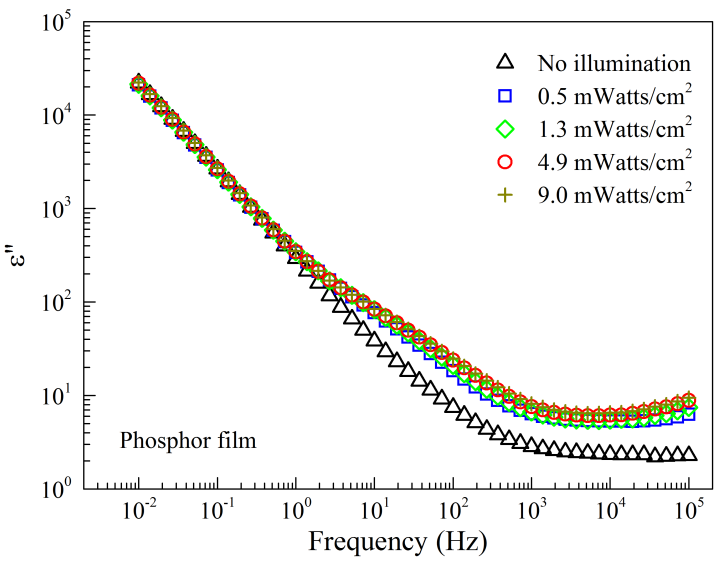

(b)

Figure 7.11: (a) (a) $\varepsilon^{\prime}$ and (b) $\varepsilon$ " as a function of frequency with the $399 \mathrm{~nm}$ illumination at different intensities for the phosphor film.

which indicates the shorter time constant of relaxation processes. This further suggest that the increased free charges assist dipoles/charges to response to the applied alternating voltage.

\section{Conductivity}

As shown in Figure 7.14, the illumination intensity also affects the $\sigma^{\prime}$ dispersions of both ACPEL devices and phosphor film. The increased intensity shifts the dispersions upwards 


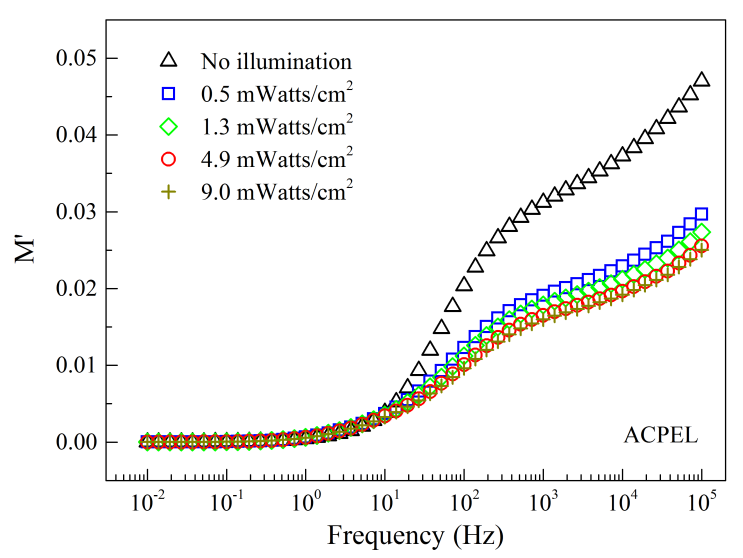

(a)

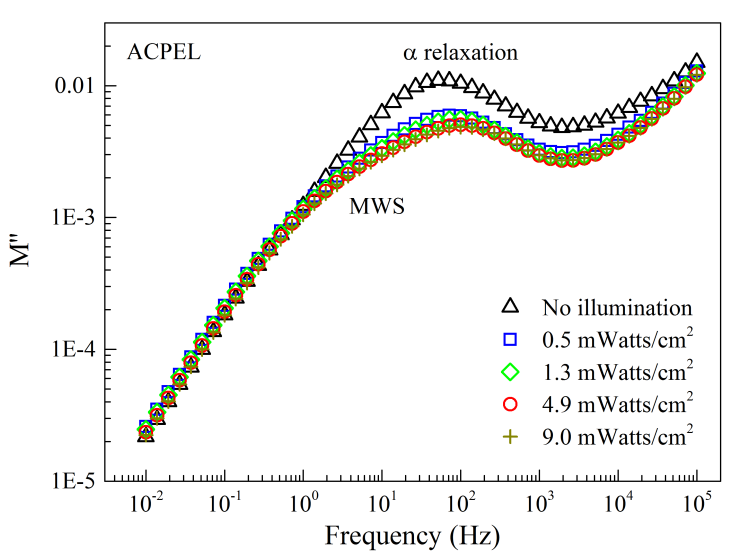

(b)

Figure 7.12: (a) $M^{\prime}$ and (b) $M^{\prime \prime}$ as a function of frequency with the $399 \mathrm{~nm}$ illumination at different intensities for ACPEL devices.

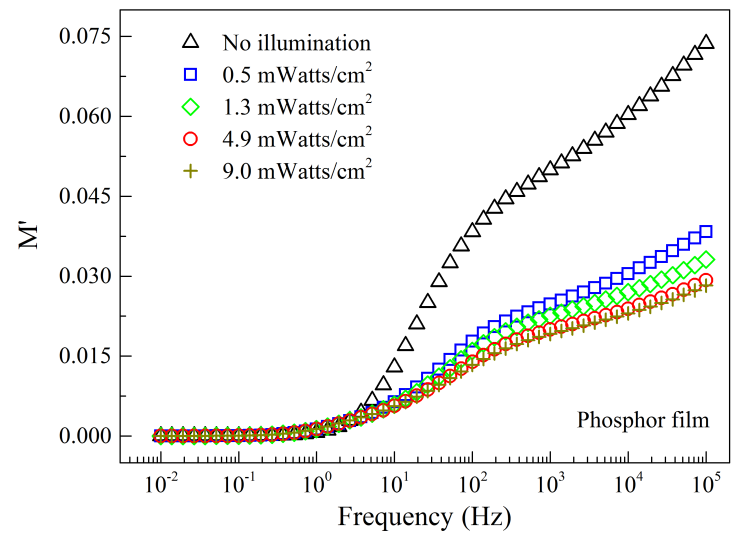

(a)

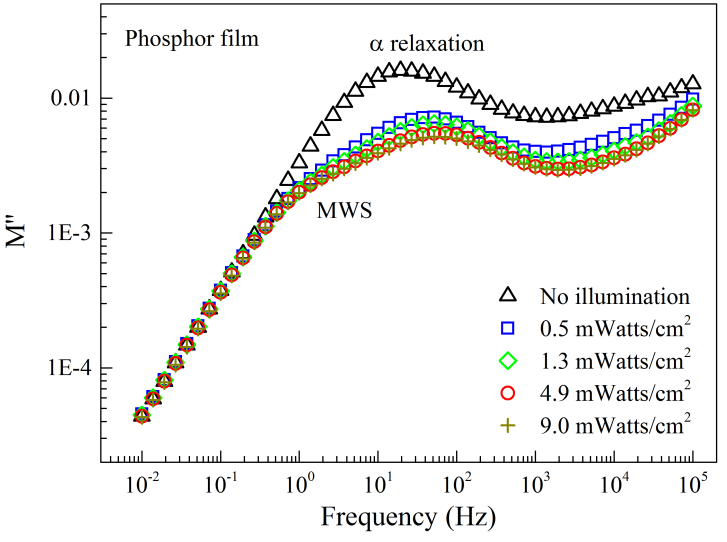

(b)

Figure 7.13: (a) (a) $M^{\prime}$ and (b) $M$ ” as a function of frequency with the $399 \mathrm{~nm}$ illumination at different intensities for the phosphor film.

at intermediate and high frequencies. The $\sigma^{\prime}$ values also tend to reach a steady state at high intensities. This effect may be due to the increased amount of free charges with the increased PL at higher illumination intensities. However, at low frequencies, the dispersion stay unaffected by the intensity. This region is the DC conductivity, which agrees well with the conclusion drawn in the previous section. Comparing Figures 7.14(a) and 7.14(b), it is observed that the phosphor film experiences higher conductivity jump with the applying of the $0.5 \mathrm{mWatts} / \mathrm{cm}^{2}$ illumination. This tells us that the conductivity of the phosphor film is 


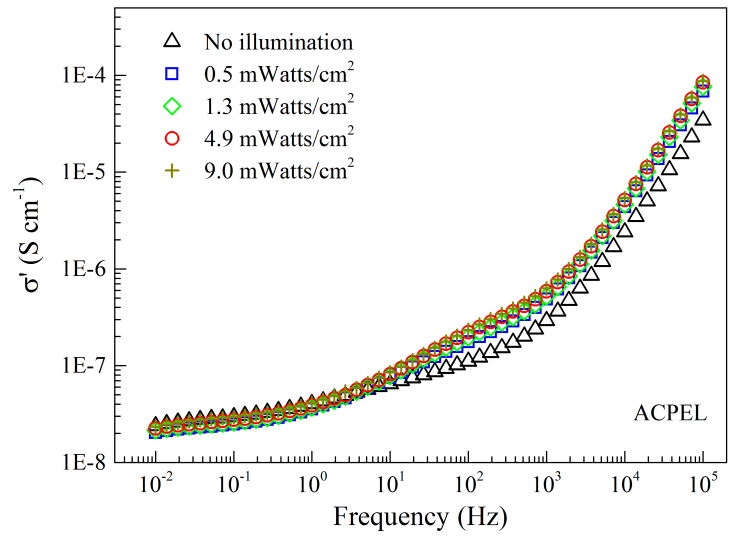

(a)

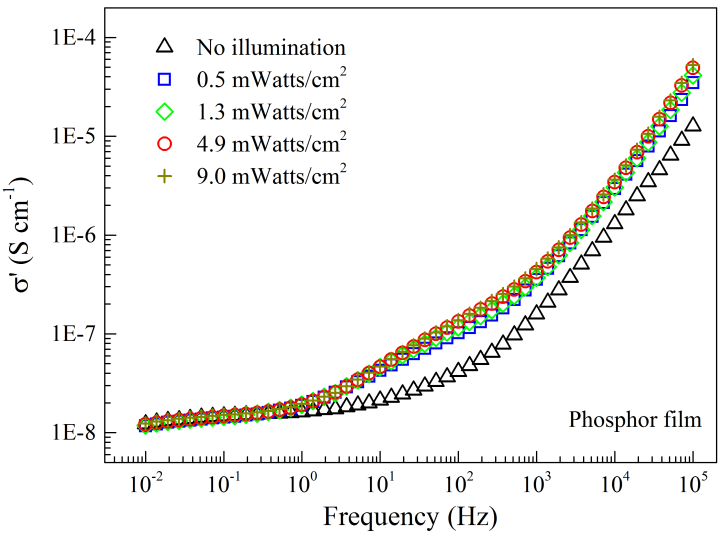

(b)

Figure 7.14: $\sigma^{\prime}$ as a function of frequency with the $399 \mathrm{~nm}$ illumination at different intensities for (a) ACPEL devices and (c) phosphor film.

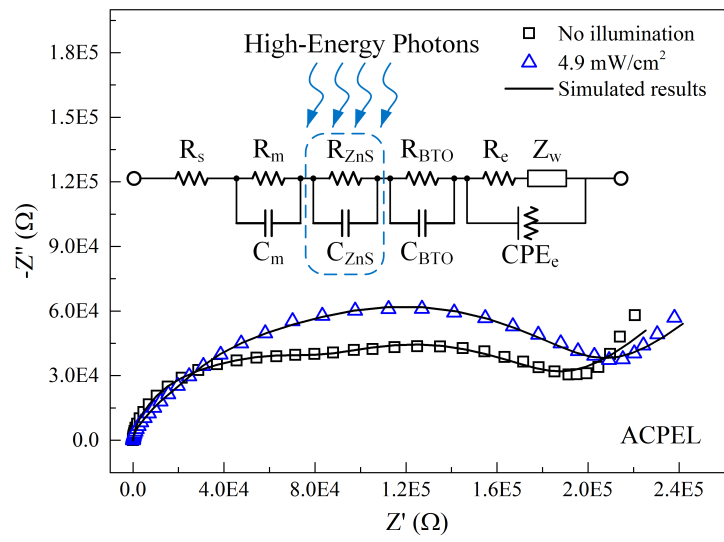

(a)

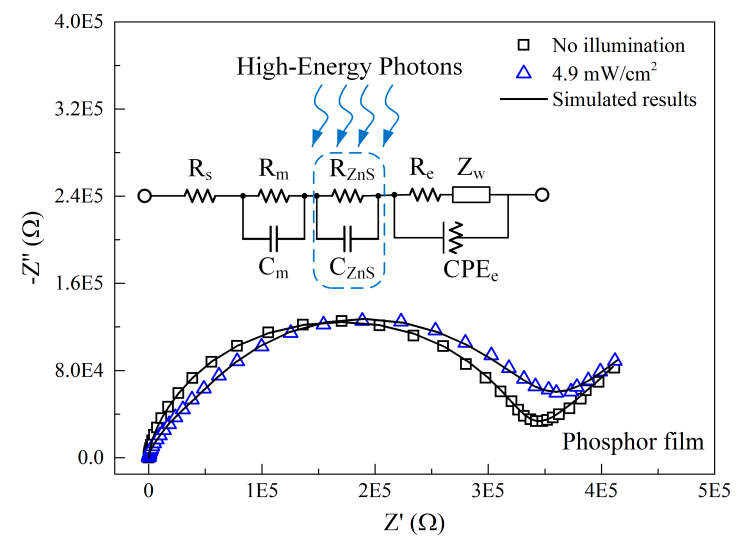

(b)

Figure 7.15: Experimental and simulated Nyquist plots of (a) ACPEL and (b) phosphor films without illumination and with $4.9 \mathrm{~mW} / \mathrm{cm} 2$ illumination together with the corresponding equivalent circuit models. High-energy photons excite the $\mathrm{ZnS}$ phosphor particles. $\mathrm{R}_{\mathrm{s}}$ : series resistance of the electrodes; $\mathrm{R}_{\mathrm{m}}$ and $\mathrm{C}_{\mathrm{m}}$ : resistance and capacitance of the binder matrix; $\mathrm{R}_{\mathrm{ZnS}}$ and $\mathrm{C}_{\mathrm{ZnS}}$ : resistance and capacitance of the $\mathrm{ZnS} /$ binder interface; $\mathrm{R}_{\mathrm{BTO}}$ and $\mathrm{C}_{\mathrm{BTO}}$ : resistance and capacitance of the $\mathrm{BaTiO}_{3}$ /binder interface; $\mathrm{R}_{\mathrm{e}}$ and $\mathrm{CPE}_{\mathrm{e}}$ : resistance and constant phase element representing EP; $\mathrm{Z}_{\mathrm{w}}$ : Warburg element.

more influenced by the intensity than the ACPEL devices. 


\subsubsection{Equivalent circuit analysis}

From the above analyses, it can be concluded that the equivalent circuits of both the ACPEL devices and phosphor films before and after the applying of a 399 illumination are identical and the increase of illumination intensity does not change the equivalent circuit formulation. Therefore, similar to the equivalent circuit proposed in Chapter 6 there are also three types of main polarization processes in photo-excited ACPEL devices, namely the EP of the electrodes, the $\alpha$ relaxation of polymer chains in the binder matrix and the MWS effect at the filler/binder interfaces. These relaxation processes are then represented in the form of equivalent circuits as shown in Figure 7.15. It is seen that the simulated Nyquist plots according to the proposed equivalent circuits for both ACPEL devices and the phosphor film fit well with the experimental data with and without applying of the $399 \mathrm{~nm}$ illumination. In the equivalent circuit model, $R_{m}$ and $C_{m}$ are the resistance and capacitance of the binder matrix representing the dipolar polarization of binder polymer chains. The MWS effect at the $\mathrm{ZnS} /$ binder and $\mathrm{BaTiO}_{3} /$ binder interfaces are modelled by two pairs of parallel resistance and capacitance: $R_{Z n S^{-}} C_{Z n S}$ and $R_{B T O}-C_{B T O}$. EP is represented by a resistance $\left(R_{e}\right)$, a Warburg element $\left(Z_{w}\right)$ and a CPE. The impedance of CPE is depicted as:

$$
Z_{\mathrm{CPE}}=\frac{1}{Y_{0 \mathrm{e}}(j 2 \pi f)^{N_{\mathrm{e}}}}
$$

where $Y_{0 e}$ is the admittance of CPE and $N_{e}$ is the degree of deviation with respect to a pure capacitor. The effective capacitance of CPE can be calculated using the following equation [221]:

$$
C_{\mathrm{e}}=Y_{0 \mathrm{e}^{1 / N_{\mathrm{e}}}} R_{\mathrm{e}}^{\left(1-N_{\mathrm{e}}\right) / N_{\mathrm{e}}}
$$

In Tables 7.1 and 7.2, $R_{Z n S}$ in the phosphor film is about two times higher than that in the EL film while the $C_{Z n S}$ values are close. This is due to the fact that the phosphor film is printed with two layers of $\mathrm{ZnS}$ particles while a ACPEL device consists of one $\mathrm{BaTiO}_{3}$ 
Table 7.1: Simulated values of the equivalent circuit components of the ACPEL devices under the $399 \mathrm{~nm}$ illumination of different intensities.

\begin{tabular}{lcccccccccccc}
\hline $\begin{array}{l}\text { Intensity } \\
\left(\mathrm{mW} / \mathrm{cm}^{2}\right)\end{array}$ & $\begin{array}{c}\mathrm{R}_{\mathrm{s}} \\
(\Omega)\end{array}$ & $\mathrm{R}_{\mathrm{m}}$ & $\mathrm{C}_{\mathrm{m}}$ & $\mathrm{R}_{\mathrm{ZnS}}$ & $\mathrm{C}_{\mathrm{ZnS}}$ & $\mathrm{R}_{\mathrm{BTO}}$ & $\mathrm{C}_{\mathrm{BTO}}$ & $\mathrm{R}_{\mathrm{e}}$ & $\mathrm{Y}_{0 \mathrm{w}}$ & $\mathrm{Y}_{0 \mathrm{e}}$ & $\mathrm{N}_{\mathrm{e}}$ & $\mathrm{C}_{\mathrm{e}}$ \\
\hline 0 & 5.29 & 3.08 & 241 & 30.1 & 110 & 32.9 & 381 & 119 & 60.5 & 2.12 & 0.738 & 1300.16 \\
0.5 & 7.16 & 4.61 & 250 & 17.5 & 294 & 28.7 & 975 & 174 & 58.5 & 1.7 & 0.781 & 1208.12 \\
1.3 & 7.54 & 4.55 & 247 & 14.8 & 347 & 29.7 & 1010 & 164 & 59.1 & 1.73 & 0.788 & 1232.69 \\
4.9 & 7.54 & 3.85 & 268 & 12.2 & 407 & 30.3 & 976 & 153 & 58.3 & 1.79 & 0.79 & 1268.64 \\
9.0 & 7.49 & 3.22 & 290 & 10.5 & 418 & 27.4 & 940 & 144 & 57.1 & 1.77 & 0.791 & 1233.43 \\
\hline
\end{tabular}

Table 7.2: Simulated values of the equivalent circuit components of the phosphor film under the $399 \mathrm{~nm}$ illumination of different intensities.

\begin{tabular}{lcccccccccc}
\hline $\begin{array}{l}\text { Intensity } \\
\left(\mathrm{mW} / \mathrm{cm}^{2}\right)\end{array}$ & $\begin{array}{c}\mathrm{R}_{\mathrm{s}} \\
(\Omega)\end{array}$ & $\begin{array}{c}\mathrm{R}_{\mathrm{m}} \\
(\mathrm{k} \Omega)\end{array}$ & $\begin{array}{c}\mathrm{C}_{\mathrm{m}} \\
(\mathrm{nF})\end{array}$ & $\begin{array}{c}\mathrm{R}_{\mathrm{ZnS}} \\
(\mathrm{k} \Omega)\end{array}$ & $\begin{array}{c}\mathrm{C}_{\mathrm{ZnS}} \\
(\mathrm{nF})\end{array}$ & $\begin{array}{c}\mathrm{R}_{\mathrm{e}} \\
(\mathrm{k} \Omega)\end{array}$ & $\begin{array}{c}\mathrm{Y}_{0 \mathrm{w}} \\
(\mu \mathrm{Mho})\end{array}$ & $\begin{array}{c}\mathrm{Y}_{0 \mathrm{e}} \\
(\mu \mathrm{Mho})\end{array}$ & $\mathrm{N}_{\mathrm{e}}$ & $\begin{array}{c}\mathrm{C}_{\mathrm{e}} \\
0\end{array}$ \\
\hline 0.56 & 11.1 & 198 & 70.6 & 128 & 248 & 34.5 & 189 & 0.866 & 117.71 \\
0.5 & 2.17 & 10.3 & 210 & 34.9 & 321 & 307 & 34.1 & 667 & 0.824 & 475.35 \\
1.3 & 3.03 & 8.17 & 225 & 27.8 & 357 & 309 & 34.1 & 643 & 0.842 & 474.80 \\
4.9 & 3.34 & 6.05 & 271 & 23.1 & 421 & 309 & 34.4 & 626 & 0.851 & 469.52 \\
9.0 & 3.39 & 5.16 & 300 & 21.3 & 436 & 305 & 36 & 621 & 0.853 & 466.19 \\
\hline
\end{tabular}

layer and one $\mathrm{ZnS}$ layer. The doubled thickness of the $\mathrm{ZnS}$ layer in the phosphor film causes the $R_{Z n S}$ value to double compare to that of the ACPEL devices. However, the surface area between $\mathrm{ZnS}$ particles and binder is also doubled, leading to similar $C_{\mathrm{ZnS}}$ values in ACPEL devices and phosphor films with $C=\varepsilon \varepsilon_{0} A / d$. As shown in Tables 7.1 and 7.2, the changes of $R_{Z n S}$ and $C_{Z n S}$ with illumination intensity exhibit similar responses with earlier studies [49, 216, 217]. When high energy photons are applied over the ACPEL devices and phosphor films, the excited $\mathrm{ZnS}$ phosphor particles lower $R_{Z n S}$ and increase $C_{Z n S}$. This is due to the 
increased photo-current at the $\mathrm{ZnS} /$ binder interfaces. These photo-excited $\mathrm{ZnS}$ phosphor particles may, in turn, affect the adjacent material represented by circuit components.

Without any illumination, $R_{m}$ in the ACPEL devices is $3.08 \mathrm{k} \Omega$ while that in the phosphor film is $11 \mathrm{k} \Omega$. With less interface ratio and higher binder content, the $\mathrm{ZnS}$ layer has a higher $R_{m}$ than the $\mathrm{BaTiO}_{3}$ layer. Thus, $R_{m}$ in the phosphor film is more than two times higher than that in the EL film. $C_{m}$ is affected by both binder volume and interaction between fillers and binder. These two factors may compensate each other, leading to a consistent $C_{m}$ value for both ACPEL devices and phosphor films. It is also observed that the resistance $\left(R_{m}\right)$ of the resin matrix decreases and the capacitance $\left(C_{m}\right)$ increases gradually with higher illumination intensity for both the ACPEL devices and phosphor films. This is attributed to the trapped charges at the $\mathrm{ZnS}$ phosphor surfaces accumulating at the $\mathrm{ZnS} /$ binder interfaces. It is possible that these charges have sufficient mobility to travel to the binder matrix and form dipoles. A higher illumination intensity will enable more trapped charges in the polymer chains and enhances the dipolar polarization.

Although it is shown that the dielectric and electrode layers are not directly affected by external illumination, both $C_{\text {ВTO }}$ in Table 7.1 and $C_{e}$ in Table 7.2 are observed to increase with intensity. As the dielectric layer is in direct contact with the $\mathrm{ZnS}$ phosphor layer in the ACPEL devices, the accumulation of charges injected from the excited $\mathrm{ZnS}$ phosphors can enhance the MWS effect at the $\mathrm{BaTiO}_{3} /$ binder interface. This causes the $C_{B T O}$ to increase with illumination intensity. The $C_{e}$ in the ACPEL devices is not affected by the photoexcited charges due to the dielectric layer sandwiched between the phosphor layer and the Ag electrode. In the phosphor film, the $\mathrm{ZnS}$ phosphor layer are in close contact with the Ag electrode, charges generated during PL are able to reach the Ag electrode and cause more charges accumulated at the $\mathrm{Ag} /$ binder interfaces, leading to the increase of $C_{e}$. The saturated $C_{e}$ values at high intensities suggest that the number of trapped charges reaches its maximum, possibly due to the limited trapping sites or mean free path of excited charges.

The admittance $Y_{0 w}$ representing the Warburg element is shown in Tables 7.1 and 7.2 


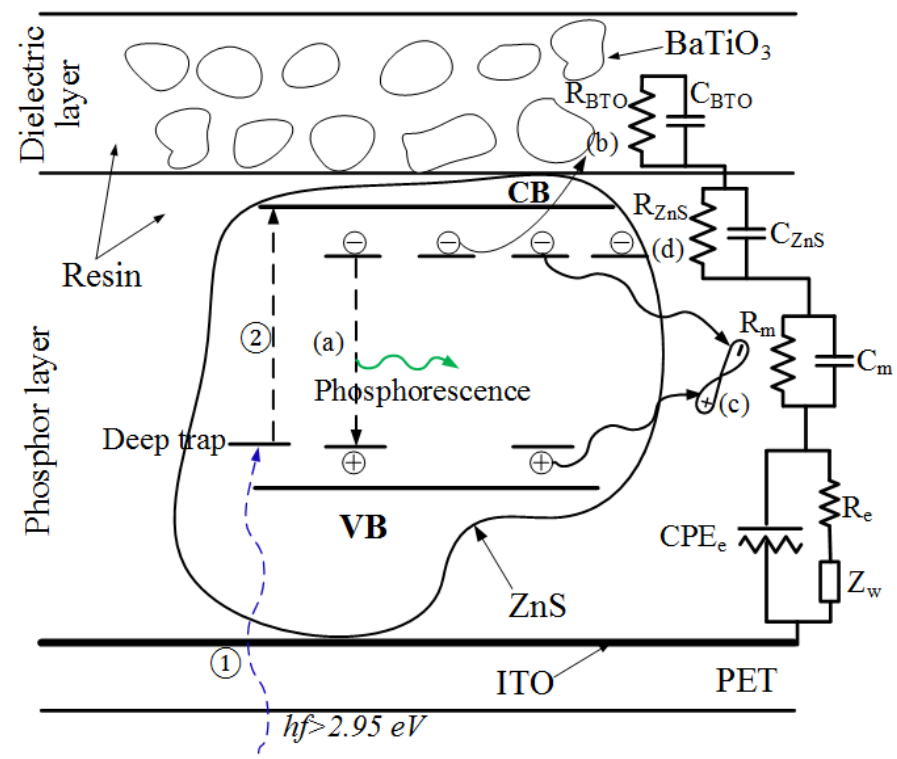

Figure 7.16: Proposed mechanism for the opto-impedance behaviors of ACPEL devices. (1) Incident photons with energy of higher than $2.95 \mathrm{eV}$ penetrate through ITO/PET and are absorbed by $\mathrm{ZnS}$ phosphor particles; (2) electrons are excited from valence band (VB) to conduction band (CB); (a) some electrons trapped in donor sites combine with holes trapped in acceptor sites and emit green phosphorescent lights; (b) some trapped charges drift and are accumulated at the $\mathrm{BaTiO}_{3}$ /binder interfaces; (c) minority charges move to the resin matrix to form dipoles; (d) other charges are trapped at the $\mathrm{ZnS} /$ resin interfaces.

for the ACPEL devices and phosphor films and does not change with illumination intensity, which indicates that the diffusional properties of ionic carriers are not affected by the photoexcited $\mathrm{ZnS}$ phosphors. It is also noted that $Y_{O_{w}}$ of the ACPEL devices is larger than that of the phosphor film. In the ACPEL devices, the Ag electrode is in contact with $\mathrm{BaTiO}_{3}$ layer while it is directly stacked over the $\mathrm{ZnS}$ phosphor layer in the phosphor film. Due to the larger surface-to-volume ratio in the dielectric layer, more ionic pathways lead to larger $Y_{O w}$.

\subsubsection{Opto-impedance mechanism}

From the above analyses on the illuminated ACPEL devices and phosphor films, a mechanism for the opto-impedance behaviors in ACPEL is proposed as illustrated in Figure 7.16. When green $\mathrm{ZnS}: \mathrm{Cu}, \mathrm{Al}$ ACPEL devices are illuminated by photons with energy higher 
than $2.95 \mathrm{eV}$, electrons in the deep trap level close to the valence band (VB) gain enough energy and are excited to the conduction band (CB) with free charges generated. Some of the free electrons trapped in donor sites have the possibility to combine with the free holes trapped in the acceptor sites to emit green phosphorescent lights. Some of the free charges generated at the surfaces of $\mathrm{ZnS}$ phosphor particles are accumulated at the $\mathrm{ZnS} / \mathrm{binder}$ interfaces leading to the enhancement of the MWS effect in the phosphor layer. For the $\mathrm{ZnS}$ particles which are in close contact or at the vicinity of $\mathrm{BaTiO}_{3}$ nanoparticles, some of the free charges at the $\mathrm{ZnS}$ surface have the possibility of hopping over and accumulated at the $\mathrm{BaTiO}_{3} /$ binder interfaces and thus enhance the corresponding MWS effect. Some other free charges that propagate to the binder matrix are trapped in the potential wells of the polymer chains and generate dipoles. This increases polarization due to the dipolar reorientation in the binder matrix. Due to the larger distance for the charges to reach binder matrix, the charging effects over the binder dipoles are small compared to the MWS effect at the $\mathrm{BaTiO}_{3} /$ binder and $\mathrm{ZnS} /$ binder interfaces.

From this opto-impedance mechanism, we can have a better idea of the charge distribution over the device level of ACPEL devices. Moreover, the low luminous efficacy is not just due to the charge loss within $\mathrm{ZnS}$ phosphors but also the charges escaping from the $\mathrm{ZnS}$ powders to the surrounding environment. From this model, a big portion of charges escape to the $\mathrm{ZnS} /$ binder and $\mathrm{BaTiO}_{3} /$ binder interfaces and small amount of charges are trapped in the binder polymer potential wells. These charge losses lead to fewer charges participating in the radiative recombination process and thus less luminous flux and luminous efficacy.

\subsection{Conclusion}

Opto-impedance spectroscopy was adopted to study the dielectric dispersion of the ACPEL devices, phosphor and dielectric films at different illumination wavelengths and intensities. High-energy photons affect the dielectric properties of the ACPEL devices and phosphor films by enhancing the MWS effect at the filler/binder interfaces and increasing the dipo- 
lar polarization of the binder matrix. This opto-impedance behavior is caused by the PL excitation of green $\mathrm{ZnS}$ phosphors during which high-energy photons ( $h f>2.95 \mathrm{eV}$ ) have sufficient energy to excite the emission centres and generate charges. When the illumination intensity increases, the MWS effect and dipolar polarization are found to increase. Equivalent circuit models of the ACPEL devices and phosphor films are constructed based on the three relaxation processes, namely EP, MWS effect at the filler/binder interfaces and dipolar polarization in the binder matrix. Through the analyses of dielectric properties and simulated equivalent circuit, a mechanism is proposed to explain the opto-impedance phenomenon in ACPEL devices. Under high energy photon illumination, electrons are first excited to the conduction bands of the $\mathrm{ZnS}$ phosphor particles, followed by being trapped in defect sites. Thereafter, these electrons have two paths: radiative and non-radiative. The radiative path enables the emission of the green phosphorescent lights. Non-radiative path consists of three possible outcomes: charge accumulation at the $\mathrm{ZnS} /$ binder interfaces and at the $\mathrm{BaTiO}_{3} /$ binder interfaces and dipole generation in the binder matrix. This model provides another perspective to understand the cause of low luminous efficacy in ACPEL devices. 


\section{Chapter 8}

\section{CONCLUSIONS AND FUTURE WORKS}

\subsection{Conclusions}

In this study, three low cost processes were developed in conjunction with screen printing process to fabricate a hybrid warm white light source, namely drop casting, wet stamping and bar coating methods. With the use of the drop casting process, F8BT was capable of downshifting blue/green lights from $\mathrm{ZnS}$ phosphors to green-yellow emissions when coated either on forward or reversed EL films. However, only those DCJTB dyes coated on reversed EL films produced downshifted red emissions due to the formation of porous structures of DCJTB nanorods stacking on the PET of forward EL films. The increase of F8BT-DCJTB mass ratio enhanced the blue and green emission peaks but reduced the red peak intensity. Diluting the F8BT and DCJTB mixed solution suppressed the yellow and red emission peaks and enhanced the blue peak intensity. White color with CIE of (0.313, 0.312) was achieved by drop casting a $1.6 \mathrm{mg} / \mathrm{mL}$ F8BT-DCJTB mixed solution with the mass ratio of 3:1 over reversed blue EL films with the operating voltage of $110 \mathrm{~V}$ and 100 Hz. The luminescence, however, was only $13.13 \mathrm{~cd} / \mathrm{m}^{2}$ with CCT of $6632 \mathrm{~K}$.

The second low cost process used in this study was a modified wet stamping method coupled with dip coating and temperature control. Through the modification of mass ratio and concentration of DCJTB-fluorescein solution, a white square hybrid EL film was fabricated with CIE of $(0.304,0.294)$ and CCT of $7162 \mathrm{~K}$ when operated at $200 \mathrm{~V}$ and 400 Hz. This hybrid film had the DCJTB-fluorescein mass ratio of 1:1 and the concentration of the mixed solution was $5 \mathrm{mg} / \mathrm{mL}$. Under this operating condition, the luminance was 93.9 $\mathrm{cd} / \mathrm{m}^{2}$ and luminance and CCT uniformities were $95.07 \%$ and $88.56 \%$. When five layers of $4 \mathrm{mg} / \mathrm{mL}$ DCJTB-fluorescein solution with the mass ratio of 1:1 were wet stamped on 
reversed green EL films, a warm white hybrid film in square shape was obtained with CCT of $2864 \mathrm{~K}$ and luminance and CCT uniformities of $93 \%$ and $93.03 \%$ when operated at 200 $\mathrm{V}$ and $400 \mathrm{~Hz}$. The luminance under this operating condition was $89.29 \mathrm{~cd} / \mathrm{m}^{2}$. To obtain a large area warm white EL film, a bar coating process was used to coat a layer of 15.0 $\mathrm{mg} / \mathrm{mL}$ DCJTB-fluorescein solution with the mass ratio of 9:1 on $100 \mathrm{~mm} \times 100 \mathrm{~mm}$ reversed green EL films. A warm white color with CCT of $2820 \mathrm{~K}$ was obtained at $200 \mathrm{~V}$ and $400 \mathrm{~Hz}$. The luminance in this condition was $72.28 \mathrm{~cd} / \mathrm{m}^{2}$ and luminance and CCT uniformities were $93.5 \%$ and $92.1 \%$.

Despite of the achievement of warm white large area EL films, its luminous efficacy was only $0.5 \mathrm{~lm} / \mathrm{W}$. Besides the luminous loss during downshifting processes, the conventional reversed green EL films had only a luminous efficacy of $3.6 \mathrm{~lm} / \mathrm{W}$. Two approaches were presented to understand this aspect of ACPEL devices. The first approach was to study the frequency dependent color shift. Green and blue EL emission spectra were each decomposed to three emission lines. The EL intensity per cycle of green emission lines decreased with frequency and tended to reach a steady level while that of blue emission lines increased with frequency first and dropped slowly at high frequencies. From literature, the luminescence centers for $\mathrm{G}-\mathrm{Cu}, \mathrm{B}-\mathrm{Cu}$ and $\mathrm{SA}$ were $\mathrm{Cu}-\mathrm{Cl}, \mathrm{Cu}_{2} \mathrm{Cl}-\mathrm{V}_{\mathrm{S}}$ and $\mathrm{V}_{\mathrm{ZnS}}-\mathrm{Cl}$ complex centers respectively. These three emissions followed recombination mechanisms of DA, Schön-Klasens and Lambe-Klick type respectively. A charge transport and relaxation model was built by considering the transport time of charge carriers and lifetime of excited impurity centers. With well fitted with the experimental results, this model attributed the low luminous efficacy to the charge relaxation and insufficient charge trapping and recombination during charge transport and recombination processes.

The second approach implemented the impedance spectroscopy methodology to assess the dielectric relaxation processes within ACPEL devices. With the analysis of frequency dependent dielectric properties at elevated temperatures in ACPEL devices, phosphor, dielectric and binder films, EP, MWS effect and dipolar polarization were found to exist 
in ACPEL devices. These relaxation processes were represented by equivalent circuit elements. Through the comparison of simulated equivalent circuit values under different illumination wavelengths and intensities, a device level charge transport and accumulation model was proposed. Apart from phosphorescence, the excited charges might accumulate at $\mathrm{ZnS} /$ binder and $\mathrm{BaTiO}_{3} /$ binder interfaces or might be trapped within binder molecules. The low luminous efficacy was thus attributed to the escaping of charges from $\mathrm{ZnS}$ phosphors to the surrounding environment.

\subsection{Future works}

In regards to the major findings of this study, several future works are recommended for the further improvement of this research work.

First, with the full understanding of the drop casting, wet stamping and bar coating processes, the downshifting material is critical in realizing a warm white hybrid EL films. Future project may focus on the development of novel red dyes or nanoparticles that are high in brightness and are stable over long period. Alternative solvents can be used to improve the printing uniformity and CRI of bar coated hybrid warm white films. A larger scale printing technique such as roll-to-roll can be implemented for the fabrication of larger warm white hybrid films.

Second, a density functional theory simulation can be conducted to verify the charge transport and relaxation model by studying the stability of G-Cu, B-Cu and SA luminescent centers. A TEM study may be carried out to investigate the material composition at the twin boundary. Space charge effects may be included in the future for the completion of this model. This model can also be extended to study the voltage-luminance relationship, waveform effect, temperature effect, degradation behaviors and decay characteristics.

Furthermore, in view of the significance of the opto-impedance methodology, it is worthwhile to investigate the change of opto-impedances during EL or light aging of the EL film. The aging study can be conducted at various temperatures, by different waveform 
or by altering operating voltage and frequency. Humidity may be another important factor. Besides, this opto-impedance methodology may be extended to study the reversed ACPEL devices, which will include the effects of the PEDOT:PSS electrode. Further performance improvement may be possible if a proper charge trapping layer is coated around the $\mathrm{ZnS}$ phosphors to prevent charge escaping. 


\section{REFERENCES}

[1] G. Nisato, D. Lupo, and S. Ganz, Organic and Printed Electronics: Fundamentals and Applications. CRC Press, 2016.

[2] T. Someya, M. Kaltenbrunner, and T. Yokota, "Ultraflexible organic electronics," MRS Bull., vol. 40, no. 12, pp. 1130-1137, 2015.

[3] J. Burroughes, D. Bradley, A. Brown, R. Marks, K Mackay, R. Friend, P. Burns, and A. Holmes, "Light-emitting diodes based on conjugated polymers," Nature, vol. 347, no. 6293, pp. 539-541, 1990.

[4] M. H. Park, T. H. Han, Y. H. Kim, S. H. Jeong, Y. Lee, H. K. Seo, H. Cho, and T. W. Lee, "Flexible organic light-emitting diodes for solid-state lighting," J. Photon. Energy, vol. 5, no. 1, pp. 053 599-053 599, 2015.

[5] Y. Sun and S. R. Forrest, "Enhanced light out-coupling of organic light-emitting devices using embedded low-index grids," Nat. Photon., vol. 2, no. 8, pp. 483-487, 2008 .

[6] L. Hung and C. Chen, "Recent progress of molecular organic electroluminescent materials and devices," Materials Science and Engineering: R: Reports, vol. 39, no. 5, pp. 143-222, 2002.

[7] A. P. Ghosh, L. J. Gerenser, C. M. Jarman, and J. E. Fornalik, "Thin-film encapsulation of organic light-emitting devices," Appl. Phys. Lett., vol. 86, no. 22, p. 223 503, 2005.

[8] K. Tseng and C. Tsou, "Novel silicon-based led packaging module with an integrated photosensing element," IEEE Photonics Technology Letters, vol. 25, no. 5, pp. 515-518, 2013.

[9] K. Xu and G. Li, "Light-emitting device with monolithic integration on bulk silicon in standard complementary metal oxide semiconductor technology," Journal of Nanophotonics, vol. 7, no. 1, pp. 073 082-073 082, 2013.

[10] D. Liu, Z. Xia, S. Cho, D. Zhao, H. Zhang, T. H. Chang, X. Yin, M. Kim, J. H. Seo, J. Lee, X. Wang, W. Zhou, and Z. Ma, "Cavity enhanced $1.5 \mathrm{~m}$ led with silicon as a hole injector," Proc. SPIE, vol. 9767, 97670Y-97670Y-6, 2016.

[11] Z. Xia, Z. Xu, M. Chen, and Q. Liu, "Recent developments in the new inorganic solid-state led phosphors," Dalton Trans., vol. 45, pp. 11 214-11 232, 282016. 
[12] J. Ohta, K. Ueno, A. Kobayashi, and H. Fujioka, "Feasibility of fabricating largearea inorganic crystalline semiconductor devices," in Intelligent Nanosystems for Energy, Information and Biological Technologies, J. Sone and S. Tsuji, Eds. Tokyo: Springer Japan, 2016, pp. 249-275.

[13] F. Holker, T. Moss, B. Griefahn, W. Kloas, C. C. Voigt, D. Henckel, A. Hnel, P. M. Kappeler, S. Volker, and A. Schwope, "The dark side of light: A transdisciplinary research agenda for light pollution policy," Ecology and Society, vol. 15, no. 4, p. $13,2010$.

[14] S. Bara, "Light pollution: Why should we care?" Proc. SPIE, vol. 9286, pp. 92862X92862X-7, 2014.

[15] T. Longcore and C. Rich, "Ecological light pollution," Frontiers in Ecology and the Environment, vol. 2, no. 4, pp. 191-198, 2004.

[16] C. Rich and T. Longcore, Ecological consequences of artificial night lighting. Island Press, 2013.

[17] T. W. Davies, J. P. Duffy, J. Bennie, and K. J. Gaston, "The nature, extent, and ecological implications of marine light pollution," Frontiers in Ecology and the Environment, vol. 12, no. 6, pp. 347-355, 2014.

[18] F. Hölker, C. Wolter, E. K. Perkin, and K. Tockner, "Light pollution as a biodiversity threat," Trends in ecology \& evolution, vol. 25, no. 12, pp. 681-682, 2010.

[19] K. J. Gaston, J. Bennie, T. W. Davies, and J. Hopkins, "The ecological impacts of nighttime light pollution: A mechanistic appraisal," Biological reviews, vol. 88, no. 4, pp. 912-927, 2013.

[20] K. J. Gaston and J. Bennie, "Demographic effects of artificial nighttime lighting on animal populations," Environmental Reviews, vol. 22, no. 4, pp. 323-330, 2014.

[21] D. M. Dominoni, "The effects of light pollution on biological rhythms of birds: An integrated, mechanistic perspective," Journal of Ornithology, vol. 156, no. 1, pp. 409-418, 2015.

[22] J. Lyytimaki, "Natures nocturnal services: Light pollution as a non-recognised challenge for ecosystem services research and management," Ecosystem Services, vol. 3, e44-e48, 2013.

[23] S. M. Pauley, "Lighting for the human circadian clock: Recent research indicates that lighting has become a public health issue," Medical Hypotheses, vol. 63, no. 4, pp. $588-596,2004$. 
[24] F. B. Cohen, C. Martinsons, F. Vienot, G. Zissis, A. B. Salsi, J. Cesarini, O. Enouf, M. Garcia, S. Picaud, and D. Attia, "Light-emitting diodes (LED) for domestic lighting: Any risks for the eye?" Progress in Retinal and Eye Research, vol. 30, no. 4, pp. $239-257,2011$.

[25] H. A. Solano Lamphar and M. Kocifaj, "Light pollution in ultraviolet and visible spectrum: Effect on different visual perceptions," PLOS ONE, vol. 8, no. 2, pp. 115, Feb. 2013.

[26] J. Bass and M. A. Lazar, "Circadian time signatures of fitness and disease," Science, vol. 354, no. 6315, pp. 994-999, 2016.

[27] D. C. Holzman, "Whats in a color? the unique human health effects of blue light," Environ Health Perspect, vol. 118, no. 1, A22-A27, 2010.

[28] F. Falchi, P. Cinzano, C. D. Elvidge, D. M. Keith, and A. Haim, "Limiting the impact of light pollution on human health, environment and stellar visibility," Journal of Environmental Management, vol. 92, no. 10, pp. 2714 -2722, 2011.

[29] X. Kang, Y. Yang, L. Wang, S. Wei, and D. Pan, "Warm white light emitting diodes with gelatin-coated AgInS 2 /ZnS core/shell quantum dots," ACS Applied Materials \& Interfaces, vol. 7, no. 50, pp. 27 713-27 719, 2015.

[30] K. J. Gaston, T. W. Davies, J. Bennie, and J. Hopkins, "Review: Reducing the ecological consequences of night-time light pollution: Options and developments," Journal of Applied Ecology, vol. 49, no. 6, pp. 1256-1266, 2012.

[31] S. M. Pawson and M. K. F. Bader, "Led lighting increases the ecological impact of light pollution irrespective of color temperature," Ecological Applications, vol. 24, no. 7, pp. 1561-1568, 2014.

[32] D. Yadav, S. Mishra, V. Shanker, and D. Haranath, "Design and development of low-power driven hybrid electroluminescent lamp from carbon nanotube embedded phosphor material," Journal of Alloys and Compounds, vol. 581, pp. 632-635, 2013.

[33] C. J. Winscom, R. Withnall, and J. Silver, "Thick film AC electroluminescence," in Handbook of Digital Imaging. John Wiley \& Sons, Ltd, 2015.

[34] M. Bredol and H. Schulze Dieckhoff, "Materials for powder-based AC-electroluminescence," Materials, vol. 3, no. 2, p. 1353, 2010.

[35] H. Kobayashi, K. Ohmi, K. Ichino, and T. Kunimoto, "Research on inorganic electroluminescence present status," Phys. status solidi A, vol. 205, no. 1, pp. 11-14, 2008. 
[36] J. H. Park, S. H. Lee, J. S. Kim, A. K. Kwon, H. L. Park, and S. Do Han, "Whiteelectroluminescent device with $\mathrm{ZnS}: \mathrm{Mn}, \mathrm{Cu}, \mathrm{Cl}$ phosphor," J. lumin., vol. 126, no. 2, pp. 566-570, 2007.

[37] B. J. Park, H. S. Seo, J. T. Ahn, J. H. Song, W. J. Chung, and D. Y. Jeon, “An investigation on photoluminescence and $\mathrm{AC}$ powder electroluminescence of $\mathrm{ZnS}: \mathrm{Cu}, \mathrm{Cl}, \mathrm{Mn}, \mathrm{Te}$ phosphor," Journal of Materials Research, vol. 26, no. 18, pp. 2394-2399, 2011.

[38] H. J. Round, “A note on carborundum,” Electronics world, vol. 49, p. 308, 1907.

[39] O. V. Lossew, "Wireless telegraphy and telephony," Telegrafia i Telefonia bez Provodor, vol. 18, p. 61, 1923.

[40] G. Destriau, "Research on the scintilation of zinc sulphide with radiation," Journal de chimie physique, vol. 33, pp. 587-625, 1936.

[41] R. Bowers and N. T. Melamed, "Luminescent centers in $\mathrm{ZnS}: \mathrm{Cu}, \mathrm{Cl}$ phosphors," Physical Review, vol. 99, no. 6, pp. 1781-1787, 1955, PR.

[42] R. H. Bube, "Electronic transitions in the luminescence of zinc sulfide phosphors," Physical Review, vol. 90, no. 1, pp. 70-80, 1953, PR.

[43] W. A. Thornton, "Electroluminescence in zinc sulfide," Physical Review, vol. 102, no. 1 , pp. 38-46, 1956, PR.

[44] P. Goldberg, "Particle size effects and the distribution of barriers in electroluminescent zinc sulfide phosphors," Journal of the Electrochemical Society, vol. 106, no. 1, pp. 34-38, 1959, Copyright 2004, IEE 1959A03335.

[45] A. G. Fischer, "Electroluminescent lines in zns powder particles. I. embedding media and basic observations," J. Electrochem. Soc., vol. 109, no. 11, pp. 1043-1049, 1962.

[46] A. G. Fischer, "Electroluminescent lines in zns powder particles. II. models and comparison with experience," J. Electrochem. Soc., vol. 110, no. 7, pp. 733-748, 1963.

[47] A. G. Fischer, "Electroluminescent iivi heterojunctions," Journal of The Electrochemical Society, vol. 118, no. 6, pp. 139C-144C, 1971.

[48] S. Kawashima, "Blue and green luminescence centers of electroluminescent zns:cu phosphors," Japanese Journal of Applied Physics, vol. 5, no. 12, pp. 1160-1169, 1966, Copyright 2004, IEE 1967A15401. 
[49] S. Roberts, "Dielectric changes of electroluminescent phosphor during illumination," Journal of the Optical Society of America, vol. 43, no. 7, pp. 590-592, 1953.

[50] S. Roberts, "Field strength and temperature studies of electroluminescent powders in dielectric media," Journal of the Optical Society of America, vol. 42, no. 11, pp. 850-854, 1952.

[51] S. Roberts, "Aging characteristics of electroluminescent phosphors," Journal of Applied Physics, vol. 28, no. 2, pp. 262-265, 1957, Copyright 2004, IEE 1957A06040.

[52] W. A. Thornton, "Electroluminescence deterioration," Journal of Applied Physics, vol. 28, no. 3, pp. 313-316, 1957, Copyright 2004, IEE 1957A05228.

[53] W. A. Thornton, "Electroluminescence maintenance," Journal of The Electrochemical Society, vol. 107, no. 11, pp. 895-907, 1960.

[54] H. Kobayashi and S. Tanaka, "The present and future prospects of electroluminescent phosphors," Journal of the Society for Information Display, vol. 4, no. 3, pp. 157-164, 1996.

[55] T. G. Ireland and J. Silver, "Studies on the orientation of ACEL ZnS:Cu particles in applied AC fields," ECS Journal of Solid State Science and Technology, vol. 3, no. 3, R25-R32, 2014.

[56] E. Vitoratos, S. Sakkopoulos, E. Dalas, N. Paliatsas, D. Karageorgopoulos, F. Petraki, S. Kennou, and S. A. Choulis, "Thermal degradation mechanisms of PEDOT:PSS," Organic Electronics, vol. 10, no. 1, pp. 61-66, 2009.

[57] V. M. Drakonakis, A. Savva, M. Kokonou, and S. A. Choulis, "Investigating electrodes degradation in organic photovoltaics through reverse engineering under accelerated humidity lifetime conditions," Solar Energy Materials and Solar Cells, vol. 130, pp. 544-550, 2014.

[58] S. Vinamrita, A. Swati, A. Manoj, S. Vishal, and R. P. Tandon, "Characterization of doped pedot: Pss and its influence on the performance and degradation of organic solar cells," Semiconductor Science and Technology, vol. 29, no. 4, p. 045020 , 2014.

[59] Y. T. Nien, I. G. Chen, C. S. Hwang, and S. Y. Chu, "Copper concentration dependence of structure, morphology and optical properties of $\mathrm{ZnS}: \mathrm{Cu}, \mathrm{Cl}$ phosphor powder," Journal of Physics and Chemistry of Solids, vol. 69, no. 2-3, pp. 366-371, 2008.

[60] Z. Szepesi, W. Lehmann, and D. Leksell, "Preparation and characteristics of white el display panels," Journal of Electronic Materials, vol. 7, no. 4, pp. 515-524, 1978. 
[61] A. Wachtel, "CaS:Cu,Eu electroluminescent phosphors," Journal of The Electrochemical Society, vol. 107, no. 3, pp. 199-206, 1960.

[62] S. Bhushan and F. S. Chandra, "AC and DC electroluminescence in CaS:Cu,Sm phosphors," Pramana, vol. 24, no. 4, pp. 575-582, 1985.

[63] S. Bhushan and F. S. Chandra, "Electroluminescense of CaS:Cu,Nd phosphors," Crystal Research and Technology, vol. 20, no. 1, K15-k18, 1985.

[64] S. Bhushan and F. S. Chandra, "Electroluminescence and photoluminescence of CaS phosphors," Journal of Physics D: Applied Physics, vol. 17, no. 3, p. 589, 1984.

[65] K. N. Kim, J. M. Kim, K. J. Choi, J. K. Park, and C. H. Kim, "Synthesis, characterization, and luminescent properties of CaS:Eu phosphor," Journal of the American Ceramic Society, vol. 89, no. 11, pp. 3413-3416, 2006.

[66] B. Car, S. Medling, C. Corrado, F. Bridges, and J. Z. Zhang, "Probing the local structure of dilute $\mathrm{Cu}$ dopants in fluorescent $\mathrm{ZnS}$ nanocrystals using EXAFS," Nanoscale, vol. 3, no. 10, pp. 4182-9, 2011.

[67] C. Corrado, Y. Jiang, F. Oba, M. Kozina, F. Bridges, and J. Z. Zhang, "Synthesis, structural, and optical properties of stable $\mathrm{ZnS}: \mathrm{Cu}, \mathrm{Cl}$ nanocrystals," The Journal of Physical Chemistry A, vol. 113, no. 16, pp. 3830-3839, 2009.

[68] N. Tri Tuan, T. Xuan Anh, N. Le Hung, and P. Thanh Huy, "Photoluminescence characteristics of as-synthesized and annealed $\mathrm{ZnS}: \mathrm{Cu}, \mathrm{Al}$ nanocrystals," Advances in Natural Sciences: Nanoscience and Nanotechnology, vol. 2, no. 3, p. 035008 , 2011.

[69] A. A. Bol, J. Ferwerda, J. A. Bergwerff, and A. Meijerink, "Luminescence of nanocrystalline $\mathrm{ZnS}: \mathrm{Cu}^{2+}$," Journal of Luminescence, vol. 99, no. 4, pp. 325-334, 2002.

[70] K. Matras, M. Bredol, A. Szatkowski, O. Sakhno, J. Stumpe, and D. Bogdal, "Composites from luminescent nanosized zns and optical polymer," Molecular Crystals and Liquid Crystals, vol. 485, pp. 776-9, 2007.

[71] V. Wood, J. E. Halpert, M. J. Panzer, M. G. Bawendi, and V. Bulovic, “Alternating current driven electroluminescence from $\mathrm{ZnSe} / \mathrm{ZnS}: \mathrm{Mn} / \mathrm{ZnS}$ nanocrystals," Nano Letters, vol. 9, no. 6, pp. 2367-2371, 2009.

[72] D. Moore and Z. L. Wang, "Growth of anisotropic one-dimensional ZnS nanostructures," Journal of Materials Chemistry, vol. 16, no. 40, pp. 3898-3905, 2006. 
[73] T. K. Tran, W. Park, W. Tong, M. M. Kyi, B. K. Wagner, and C. J. Summers, "Photoluminescence properties of ZnS epilayers," Journal of Applied Physics, vol. 81, no. 6, pp. 2803-2809, 1997.

[74] C.-Y. Yeh, Z. W. Lu, S. Froyen, and A. Zunger, "Zinc-blende wurtzite polytypism in semiconductors," Physical Review B, vol. 46, no. 16, pp. 10 086-10 097, 1992.

[75] H. Chen, D. Shi, J. Qi, J. Jia, and B. Wang, "The stability and electronic properties of wurtzite and zinc-blende ZnS nanowires," Physics Letters A, vol. 373, no. 3, pp. 371-375, 2009.

[76] K. C. Sharma and Y. A. Chang, “The S-Zn (sulfur-zinc) system," Journal of Phase Equilibria, vol. 17, no. 3, pp. 261-266, 1996.

[77] S. Shionoya, T. Koda, K. Era, and H. Fujiwara, "Nature of luminescence transitions in ZnS crystals," Journal of the Physical Society of Japan, vol. 19, no. 7, pp. 11571167, 1964.

[78] N. Yung Tang, C. In Gann, H. Chii Shyang, and C. Sheng Yuan, "Microstructure and electroluminescence of $\mathrm{ZnS}: \mathrm{Cu}, \mathrm{Cl}$ phosphor powders prepared by firing with CuS nanocrystallites," Journal of Electroceramics, vol. 17, no. 2-4, pp. 299-303, 2006.

[79] R. Withnall, J. Silver, T. G. Ireland, G. R. Fern, and P. J. Marsh, "Structure and morphology of ACEL ZnS:Cu,Cl phosphor powder etched by hydrochloric acid," Journal of The Electrochemical Society, vol. 156, no. 11, J326-J332, 2009.

[80] A. C. Wright and I. V. F. Viney, "A structural evaluation of ZnS-based electroluminescent phosphors and devices: A comparison with performance," Philosophical Magazine Part B, vol. 81, no. 3, pp. 279-297, 2001.

[81] P. Brovetto, V. Maxia, and C. Muntoni, "Kinetics of a.c. electroluminescence in ZnS," Il Nuovo Cimento B (1965-1970), vol. 69, no. 2, pp. 219-240, 1970.

[82] G. F. Neumark, "Defects in wide band gap II-VI crystals," Materials Science and Engineering: R: Reports, vol. 21, no. 1, pp. iii-46, 1997.

[83] L. L. Chang, L. Esaki, and R. Tsu, "Vacancy association of defects in annealed GaAs," Applied Physics Letters, vol. 19, no. 5, pp. 143-145, 1971.

[84] G. D. Watkins, "The identity of the EPR A-center and the self-activated luminescence center in ZnS:Cl," Solid State Communications, vol. 12, no. 7, pp. 589-592, 1973. 
[85] A. N. Georgobiani, R. G. Maev, Y. V. Ozerov, and E. E. Strumban, "Investigation of deep centres of chlorine-doped zinc sulfide crystals," Physica status solidi (a), vol. 38 , no. 1, pp. 77-83, 1976.

[86] N. A. Vlasenko, M. M. Chumachkova, Z. L. Denisova, and L. I. Veligura, "On nature of centers responsible for inherent memory in ZnS:Mn thin-film electroluminescent devices," Journal of Crystal Growth, vol. 216, no. 14, pp. 249-255, 2000.

[87] J. C. Hitt, J. P. Bender, and J. F. Wager, "Thin-film electroluminescent device physics modeling," Critical Reviews in Solid State and Materials Sciences, vol. 25, no. 1, pp. 29-85, 2000.

[88] K. Matsuura, S. Kishida, and I. Tsurumi, "Annealing of the F+ EPR centers and the 2.3 and 2.9 ev optical absorption bands in zinc sulfide crystals," Physica status solidi (b), vol. 140, no. 2, pp. 347-354, 1987.

[89] I. S. Hauksson, J. Simpson, S. Y. Wang, K. A. Prior, and B. C. Cavenett, "Compensation processes in nitrogen doped ZnSe," Applied Physics Letters, vol. 61, no. 18, pp. 2208-2210, 1992.

[90] W. Faschinger, "Fundamental doping limits in wide gap II-VI compounds," Journal of Crystal Growth, vol. 159, no. 1, pp. 221-228, 1996.

[91] S. B. Zhang, S. H. Wei, and A. Zunger, "Intrinsic $n$-type versus $p$-type doping asymmetry and the defect physics of ZnO," Physics Review B, vol. 63, p. 075 205, 72001.

[92] J. Stanley, Y. Jiang, F. Bridges, S. A. Carter, and L. Ruhlen, "Degradation and rejuvenation studies of AC electroluminescent $\mathrm{ZnS}: \mathrm{Cu}, \mathrm{Cl}$ phosphors," Journal of Physics Condensed Matter, vol. 22, no. 5, p. 055 301, 2010.

[93] A. Suzuki and S. Shionoya, "Mechanism of the green-copper luminescence in $\mathrm{ZnS}$ crystals. I. direct evidence for the pair emission mechanism," Journal of the Physical Society of Japan, vol. 31, no. 5, pp. 1455-1461, 1971.

[94] K. Urabe, S. Shionoya, and A. Suzuki, "Polarization of the blue-copper luminescence in ZnS crystals," Journal of the Physical Society of Japan, vol. 25, no. 6, pp. 1611-1617, 1968.

[95] W. Kaiser, R. P. Marques, and A. F. Correa, "Light emission of electroluminescent lamps under different operating conditions," in 2012 IEEE Industry Applications Society Annual Meeting, 7-11 Oct. 2012, ser. 2012 IEEE Industry Applications Society Annual Meeting, IEEE, 8 pp. 
[96] N. E. Grzeskowiak and J. F. Winkel, "Structure and location of electroluminescent light emission within $\mathrm{ZnS} / \mathrm{Cu}$ ACEL powder phosphor particles," Journal of the Electrochemical Society, vol. 154, no. 10, pp. 289-94, 2007.

[97] S. Kawashima, "Effect of polarization field on electroluminescence," Journal of the Electrochemical Society, vol. 113, no. 10, pp. 1083-1085, 1966.

[98] C. X. Guo, S. Y. Zhang, B. L. Li, and L. Xu, "Microscopic structure and distribution of $\mathrm{Cu}$ in the electroluminescence phosphor $\mathrm{ZnS}: \mathrm{Cu}$," Journal of Luminescence, vol. 40-41, pp. 782-783, 1987.

[99] M. Warkentin, F. Bridges, S. A. Carter, and M. Anderson, "Electroluminescence materials $\mathrm{ZnS}: \mathrm{Cu}, \mathrm{Cl}$ and $\mathrm{ZnS}: \mathrm{Cu}, \mathrm{Mn}, \mathrm{Cl}$ studied by EXAFS spectroscopy," Physical Review B, vol. 75, no. 7, p. 075 301, 2007.

[100] G. Busca, C. Cortese, and V. Maxia, "Distribution of bringhtness along the electroluminescent patterns in ZnS," Lettere al Nuovo Cimento, vol. 2, no. 5, pp. 201-204, 1971.

[101] V. Maxia, C. Muntoni, and M. Murgia, "Effect of trapped electrons on the buildingup of electroluminescence," Lettere al Nuovo Cimento, vol. 1, no. 11, pp. 457-463, 1971.

[102] V. Maxia, C. Muntoni, and M. Murgia, "Frequency dependence of the brightnessvoltage relationship in electroluminescent ZnS," Lettere al Nuovo Cimento, vol. 2, no. 6, pp. 312-316, 1971.

[103] S. Ganz, H. Sauer, S. Weienseel, J. Zembron, R. Tone, E. Drsam, M. Schaefer, and M. Schulz Ruthenberg, "Printing and processing techniques," in Organic and Printed Electronics. Pan Stanford, 2016, pp. 47-116.

[104] F. C. Krebs, "Fabrication and processing of polymer solar cells: A review of printing and coating techniques," Solar Energy Materials and Solar Cells, vol. 93, no. 4, pp. $394-412$, 2009, Processing and Preparation of Polymer and Organic Solar Cells.

[105] H. Yang and P. Jiang, "Large-scale colloidal self-assembly by doctor blade coating," Langmuir, vol. 26, no. 16, pp. 13 173-13 182, 2010.

[106] C. S. McCamy, "Correlated color temperature as an explicit function of chromaticity coordinates," Color Research \& Application, vol. 17, no. 2, pp. 142-144, 1992.

[107] P. A. Tipler and G. Mosca, Physics for scientists and engineers : With modern physics. New York : W.H. Freeman, c2008. 6th, [extended]., 2008. 
[108] K. S. Cole and R. H. Cole, "Dispersion and absorption in dielectrics I. alternating current characteristics," The Journal of Chemical Physics, vol. 9, no. 4, pp. 341$351,1941$.

[109] G. M. Tsangaris, G. C. Psarras, and N. Kouloumbi, "Electric modulus and interfacial polarization in composite polymeric systems," Journal of Materials Science, vol. 33, no. 8, pp. 2027-2037, 1998.

[110] Y. Li and S. Mao, "Study on the properties and application of epoxy resin/polyurethane semi-interpenetrating polymer networks," Journal of Applied Polymer Science, vol. 61, no. 12, pp. 2059-2063, 1996.

[111] G. C. Psarras, "Hopping conductivity in polymer matrixmetal particles composites," Composites Part A: Applied Science and Manufacturing, vol. 37, no. 10, pp. 1545-1553, 2006.

[112] W. Jilani, N. Mzabi, O. Gallot Lavallee, N. Fourati, R. Zerrouki, and H. Guermazi, "Dielectric characterization of epoxy resin based polymer," in Troisime rencontre francophone sur les matriaux isolants, 2014.

[113] A. Soulintzis, G. Kontos, P. Karahaliou, G. C. Psarras, S. N. Georga, and C. A. Krontiras, "Dielectric relaxation processes in epoxy resinZnO composites," Journal of Polymer Science Part B: Polymer Physics, vol. 47, no. 4, pp. 445-454, 2009.

[114] R. Casalini, D. Fioretto, A. Livi, M. Lucchesi, and P. A. Rolla, "Influence of the glass transition on the secondary relaxation of an epoxy resin," Physical Review B, vol. 56, no. 6, pp. 3016-3021, 1997.

[115] P. Ajayan, L. Schadler, and P. Braun, Nanocomposite science and technology. WileyVCH, 2003.

[116] H. Althues, J. Henle, and S. Kaskel, "Functional inorganic nanofillers for transparent polymers," Chemical Society Reviews, vol. 36, no. 9, pp. 1454-1465, 2007.

[117] Y. Bai, Z.-Y. Cheng, V. Bharti, H. S. Xu, and Q. M. Zhang, "High-dielectricconstant ceramic-powder polymer composites," Applied Physics Letters, vol. 76, no. 25 , pp. 3804-3806, 2000.

[118] J. Maxwell, A treatise on electricity and magnetism, ser. A Treatise on Electricity and Magnetism v. 1. Clarendon Press, 1873.

[119] K. W. Wagner, "Erklärung der dielektrischen nachwirkungsvorgänge auf grund maxwellscher vorstellungen," Archiv für Elektrotechnik, vol. 2, no. 9, pp. 371-387, 1914. 
[120] R. W. Sillars, "The properties of a dielectric containing semiconducting particles of various shapes," Electrical Engineers, Journal of the Institution of, vol. 80, no. 484, pp. 378-394, 1937.

[121] L. Cheng, L. Zheng, G. Li, J. Zeng, and Q. Yin, "Influence of particle surface properties on the dielectric behavior of silica/epoxy nanocomposites," Physica B: Condensed Matter, vol. 403, no. 17, pp. 2584 -2589, 2008.

[122] G. Polizos, V. Tomer, E. Manias, and C. A. Randall, "Epoxy-based nanocomposites for electrical energy storage. II: Nanocomposites with nanofillers of reactive montmorillonite covalently-bonded with barium titanate," Journal of Applied Physics, vol. 108, no. 7, 074117, 2010.

[123] B. Chu, X. Zhou, K. Ren, B. Neese, M. Lin, Q. Wang, F. Bauer, and Q. M. Zhang, "A dielectric polymer with high electric energy density and fast discharge speed," Science, vol. 313, no. 5785, pp. 334-336, 2006.

[124] T. J. Lewis, "Interfaces: Nanometric dielectrics," Journal of Physics D: Applied Physics, vol. 38, no. 2, p. 202, 2005.

[125] R. Maity, U. Maiti, M. Mitra, and K. Chattopadhyay, "Synthesis and optical characterization of polymer-capped nanocrystalline $\mathrm{ZnS}$ thin films by chemical process," Physica E: Low-dimensional Systems and Nanostructures, vol. 33, no. 1, pp. 104 $-109,2006$.

[126] S. A. FitzGerald, T. W. Noh, A. J. Sievers, L. A. Xue, and Y. Tzou, "Far-infrared investigation of the generalized Lyddane-Sachs-Teller relation using ZnS-diamond composites," Phys. Rev. B, vol. 42, pp. 5469-5475, 91990.

[127] H. Wei, X. Yan, Y. Li, S. Wu, A. Wang, S. Wei, and Z. Guo, "Hybrid electrochromic fluorescent Poly(DNTD)/CdSe/ZnS composite films," The Journal of Physical Chemistry $C$, vol. 116, no. 7, pp. 4500-4510, 2012.

[128] B. Thomas and M. Abdulkhadar, "Dielectric properties of nano-particles of zinc sulphide," Pramana, vol. 45, no. 5, pp. 431-438, 1995.

[129] P. K. Ghosh, M. K. Mitra, and K. K. Chattopadhyay, "Zns nanobelts grown in a polymer matrix by chemical bath deposition," Nanotechnology, vol. 16, no. 1, p. 107, 2005.

[130] X. L, N. L, J. Gao, X. Jin, and C. L, "Synthesis and properties of ZnS/polyimide nanocomposite films," Polymer International, vol. 56, no. 5, pp. 601-605, 2007. 
[131] K. Chakraborty, P. Das, S. Chakrabarty, T. Pal, and S. Ghosh, "AC conduction and timetemperature superposition scaling in a reduced graphene oxide-zinc sulfide nanocomposite," ChemPhysChem, vol. 17, no. 10, pp. 1518-1523, 2016.

[132] J. Bessiere, K. Chlihi, J. M. Thiebaut, and G. Roussy, "Dielectric study of the activation and deactivation of sphalerite by metallic ions," International Journal of Mineral Processing, vol. 28, no. 1, pp. 1-13, 1990.

[133] H. C. Pant, M. K. Patra, S. C. Negi, A. Bhatia, S. R. Vadera, and N. Kumar, "Studies on conductivity and dielectric properties of polyaniline-zinc sulphide composites," Bulletin of Materials Science, vol. 29, no. 4, pp. 379-384, 2006.

[134] Y. P. Mao, S. Y. Mao, Z. G. Ye, Z. X. Xie, and L. S. Zheng, "Size-dependences of the dielectric and ferroelectric properties of $\mathrm{BaTio}_{3} /$ polyvinylidene fluoride nanocomposites," Journal of Applied Physics, vol. 108, no. 1, 014102, 2010.

[135] J. Kim, T. Noh, S. Jeon, S. Park, H. Chun, and H. Lee, "Deterioration behavior analysis of dysprosium and thulium co-doped barium titanate ceramics for multilayer ceramic capacitors," Ceramics International, vol. 38, no. 8, pp. 6837-6842, 2012.

[136] S. H. Yoon, S. H. Kang, S. H. Kwon, and K. H. Hur, "Resistance degradation behavior of Ca-doped $\mathrm{BaTiO}_{3}$," Journal of Materials Research, vol. 25, no. 11, pp. 2135-2145, 2010.

[137] T. Hanemann, H. Gesswein, and B. Schumacher, "Development of new polymer$\mathrm{BaTiO}_{3}$-composites with improved permittivity for embedded capacitors," Microsystem Technologies, vol. 17, no. 2, pp. 195-201, 2011.

[138] M. H. Frey and D. A. Payne, "Grain-size effect on structure and phase transformations for barium titanate," Phys. Rev. B, vol. 54, pp. 3158-3168, 51996.

[139] B. H. Fan, J. W. Zha, D. R. Wang, J. Zhao, and Z. M. Dang, "Experimental study and theoretical prediction of dielectric permittivity in $\mathrm{BaTiO}_{3} /$ polyimide nanocomposite films," Applied Physics Letters, vol. 100, no. 9, 092903, 2012.

[140] Z. M. Dang, D. Xie, and C. Y. Shi, "Theoretical prediction and experimental study of dielectric properties in poly(vinylidene fluoride) matrix composites with micronanosize $\mathrm{BaTiO}_{3}$ filler," Applied Physics Letters, vol. 91, no. 22, $222902,2007$.

[141] Z. M. Dang, Y. F. Yu, H. P. Xu, and J. Bai, "Study on microstructure and dielectric property of the $\mathrm{BaTiO}_{3}$ /epoxy resin composites," Composites Science and Technology, vol. 68, no. 1, pp. $171-177,2008$. 
[142] S. F. Mendes, C. M. Costa, C. Caparros, V. Sencadas, and S. Lanceros-Méndez, "Effect of filler size and concentration on the structure and properties of poly(vinylidene fluoride)/ $/ \mathrm{BaTiO}_{3}$ nanocomposites," Journal of Materials Science, vol. 47, no. 3, pp. 1378-1388, 2012.

[143] L. Ramajo, M. Reboredo, and M. Castro, "Dielectric response and relaxation phenomena in composites of epoxy resin with $\mathrm{BaTiO}_{3}$ particles," Composites Part A: Applied Science and Manufacturing, vol. 36, no. 9, pp. 1267 -1274, 2005.

[144] W. Yang, S. Yu, S. Luo, R. Sun, W. H. Liao, and C. P. Wong, "A systematic study on electrical properties of the $\mathrm{BaTiO}_{3}$-epoxy composite with different sized $\mathrm{BaTiO}_{3}$ as fillers," Journal of Alloys and Compounds, vol. 620, pp. 315-323, 2014.

[145] A Patsidis and G. C Psarras, "Dielectric behaviour and functionality of polymer matrixceramic $\mathrm{BaTiO}_{3}$ composites," Currents, vol. 5, p. 10, 2008.

[146] Y. Baziard, S. Breton, S. Toutain, and A. Gourdenne, "Dielectric properties of aluminium powder-epoxy resin composites," European Polymer Journal, vol. 24, no. 6, pp. 521-526, 1988.

[147] Z. Wang, W. Zhou, L. Dong, X. Sui, H. Cai, J. Zuo, and Q. Chen, "Dielectric spectroscopy characterization of relaxation process in Ni/epoxy composites," Journal of Alloys and Compounds, vol. 682, pp. 738-745, 2016.

[148] G. C. Psarras, E. Manolakaki, and G. M. Tsangaris, "Electrical relaxations in polymeric particulate composites of epoxy resin and metal particles," Composites Part A: Applied Science and Manufacturing, vol. 33, no. 3, pp. 375-384, 2002.

[149] X. Zhang, G. Liang, J. Chang, A. Gu, L. Yuan, and W. Zhang, "The origin of the electric and dielectric behavior of expanded graphitecarbon nanotube/cyanate ester composites with very high dielectric constant and low dielectric loss," Carbon, vol. 50, no. 14, pp. 4995-5007, 2012.

[150] J. Wu, Z. Li, and Y. Lin, "Porous nio/ag composite film for electrochemical capacitor application," Electrochimica Acta, vol. 56, no. 5, pp. 2116 -2121, 2011.

[151] Y. Cha, M. Aureli, and M. Porfiri, "A physics-based model of the electrical impedance of ionic polymer metal composites," Journal of Applied Physics, vol. 111, no. 12, p. $124901,2012$.

[152] J. K. J. Van-Duren, X. Yang, J. Loos, C. W. T. Bulle-Lieuwma, A. B. Sieval, J. C. Hummelen, and R. A. J. Janssen, "Relating the morphology of poly(p-phenylene vinylene)/methanofullerene blends to solar-cell performance," Advanced Functional Materials, vol. 14, no. 5, pp. 425-434, 2004. 
[153] A. C. Morteani, A. S. Dhoot, J. S. Kim, C. Silva, N. C. Greenham, C. Murphy, E. Moons, S. Cina, J. H. Burroughes, and R. H. Friend, "Barrier-free electronhole capture in polymer blend heterojunction light-emitting diodes," Advanced Materials, vol. 15, no. 20, pp. 1708-1712, 2003.

[154] M. Ding, "High-power single-chip GaN-based white LED with 3058 lm," Electronics Letters, vol. 52, 2050-2051(1), 252016.

[155] J. Sun Park, B. Ram Lee, E. Jeong, H. J. Lee, J. Min Lee, J. S. Kim, J. Young Kim, H. Young Woo, S. Ouk Kim, and M. Hoon Song, "High performance polymer lightemitting diodes with N-type metal oxide/conjugated polyelectrolyte hybrid charge transport layers," Applied Physics Letters, vol. 99, no. 16, p. 163 305, 2011.

[156] C. Jie Rong, W. Xue Yan, and W. Tomiji, "Wettability of poly(ethylene terephthalate) film treated with low-temperature plasma and their surface analysis by ESCA," Journal of Applied Polymer Science, vol. 72, no. 10, pp. 1327-1333, 1999.

[157] X. Y. Zheng, W. Q. Zhu, Y. Z. Wu, X. Y. Jiang, R. G. Sun, Z. L. Zhang, and S. H. $\mathrm{Xu}$, "A white OLED based on DPVBi blue light emitting host and DCJTB red dopant," Displays, vol. 24, no. 3, pp. 121-124, 2003.

[158] S. Zhang, R. J. W. Teo, H. Su, C. S. Tan, and T. K. S. Wong, "Color tunable hybrid AC powder electroluminescent devices with organic fluorescent materials," Opt. Mater. Express, vol. 6, no. 9, pp. 2879-2891, 2016.

[159] J. T. Miao, C. Fan, X. Y. Shi, R. Sun, Y. J. Xu, and J. F. Ge, "Colorimetric and ratiometric $\mathrm{pH}$ responses by the protonation of phenolate within hemicyanine," Analyst, vol. 139, no. 23, pp. 6290-6297, 2014.

[160] C. I. Zovko and T. C. Nerz, "White polymer thick film electroluminescent lamps and their applications for backlighting liquid crystal displays in portable electronic devices," Displays, vol. 20, no. 3, pp. 155-159, 1999.

[161] J. Y. Kim, H. Kim, D. Jung, and S. Yu, "Enhanced electroluminescence performances by controlling the position of carbon nanotubes," Journal of Applied Physics, vol. 112, no. 10, p. $104515,2012$.

[162] F.-H. Wang, K.-F. Chen, Y.-H. Chien, C.-C. Chang, and M.-Y. Chuang, "Enhanced luminance for inorganic electroluminescent devices with a charged electret," Journal of Luminescence, vol. 141, pp. 106 -110, 2013.

[163] S. Park, J. H. Kim, M. K. Lee, J. Lee, Y. C. Kim, and J. C. Lee, "Effect of solution combusted $\mathrm{TiO}_{2}$ nanoparticles added to the dielectric layers for low power consumption AC powder electroluminescence devices," Journal of nanoscience and nanotechnology, vol. 14, no. 8, pp. 6120-6123, 2014. 
[164] F. H. Wang and K. F. Chen, "Efficiency enhancement of flexible inorganic powder electroluminescent devices using the $\mathrm{BaTiO}_{3}$-MWNT composite dielectric layer," Journal of Display Technology, vol. 12, no. 11, pp. 1383-1387, 2016.

[165] A. Sadakata, Y. Ohshima, D. Taguchi, M. Fukuzawa, T. Manaka, and M. Iwamoto, "Electroluminescence enhanced from electrode interface in ITO / Tetracene / Al diodes," Molecular Crystals and Liquid Crystals, vol. 538, no. 1, pp. 112-117, 2011.

[166] J. Ibanez, E. Garcia, L. Gil, M. Mollar, and B. Mari, "Frequency-dependent light emission and extinction of electroluminescent ZnS:cu phosphor," Displays, vol. 28, no. 3, pp. 112-117, 2007.

[167] J. Prener and F. Williams, "Theorie des centres luminogenes du type donneuraccepteur associes," J. Phys. Radium, vol. 17, no. 8-9, pp. 667-672, 1956.

[168] H. Haupt and H. Nelkowski, "Explanation of the comet shape of electroluminescent lines in $\mathrm{ZnS}: \mathrm{Cu}$ single crystals," Journal of Luminescence, vol. 16, no. 3, pp. 251$5,1978$.

[169] C. X. Guo, S. Y. Zhang, B. L. Li, and L. Xu, "Microscopic structure and distribution of $\mathrm{Cu}$ in the electroluminescence phosphor $\mathrm{ZnS}$ :Cu," Journal of Luminescence, vol. 40-1, pp. 782-783, 1988.

[170] S. Rothschild, "The luminescent spectra of zinc sulphide and zinc cadmium sulphide phosphors," Transactions of the Faraday Society, vol. 42, no. 0, pp. 635642, 1946.

[171] F. A. Kröger, J. E. Hellingman, and N. W. Smit, "The fluorescence of zinc sulphide activated with copper," Physica, vol. 15, no. 11, pp. 990-1018, 1949.

[172] S. Shionoya, K. Urabe, T. Koda, K. Era, and H. Fujiwara, "Nature of the red-copper luminescence centre in $\mathrm{ZnS}$ crystals as elucidated by polarization measurements," Journal of Physics and Chemistry of Solids, vol. 27, no. 5, pp. 865-869, 1966.

[173] N. T. Melamed, "Sulfur vacancy emission in ZnS phosphors," Physical Review, vol. 107, no. 6, pp. 1727-1728, 1957.

[174] J. S. Prener and F. E. Williams, "Activator systems in zinc sulfide phosphors," Journal of The Electrochemical Society, vol. 103, no. 6, pp. 342-346, 1956.

[175] W. C. Holton, M. De Wit, R. K. Watts, T. L. Estle, and J. Schneider, "Paramagnetic copper centers in ZnS," Journal of Physics and Chemistry of Solids, vol. 30, no. 4, pp. 963-977, 1969. 
[176] E. F. Apple and J. S. Prener, "On the infrared emission in ZnS:Cu-effect of sulfur pressure and aluminum," Journal of Physics and Chemistry of Solids, vol. 13, no. 1, pp. 81-87, 1960.

[177] A. Suzuki and S. Shionoya, "Mechanism of the green-copper luminescence in $\mathrm{ZnS}$ crystals. II. polarization characteristics," Journal of the Physical Society of Japan, vol. 31, no. 5, pp. 1462-1468, 1971.

[178] T. Koda and S. Shionoya, "Nature of the self-activated blue luminescence center in cubic ZnS:Cl single crystals," Physical Review, vol. 136, no. 2A, A541-A555, 1964, PR.

[179] H. A. Klasens, "On the nature of fluorescent centers and traps in zinc sulfide," Journal of The Electrochemical Society, vol. 100, no. 2, pp. 72-80, 1953.

[180] J. S. Prener and F. E. Williams, "Selfactivation and selfcoactivation in zinc sulfide phosphors," The Journal of Chemical Physics, vol. 25, no. 2, pp. 361-361, 1956.

[181] T. Koda and S. Shionoya, "Optical symmetry of the self-activated luminescence center in ZnS single crystal," Physical Review Letters, vol. 11, no. 2, pp. 77-80, 1963, PRL.

[182] R. T. Williams and K. S. Song, "The self-trapped exciton," Journal of Physics and Chemistry of Solids, vol. 51, no. 7, pp. 679-716, 1990.

[183] K. S. Song and R. C. Baetzold, "Structure of the self-trapped exciton and nascent frenkel pair in alkali halides: An ab initio study," Physical Review B, vol. 46, no. 4, pp. 1960-1969, 1992, PRB.

[184] C. R. Fu, L. F. Chen, and K. S. Song, "Self-trapped excitons in pure and Na- and Tldoped caesium halides and the recombination luminescence," Journal of Physics: Condensed Matter, vol. 11, no. 28, p. 5517, 1999.

[185] S. Shigeo, E. Koh, and K. Hirohiko, "Decay characteristics of luminescence in ZnS phosphors by pulse light excitation," Journal of Physics and Chemistry of Solids, vol. 26, no. 4, pp. 697-709, 1965.

[186] H. Blicks, N. Riehl, and R. Sizmann, "Reversible leuchtzentren-umwandlungen in ZnS-phosphoren," Zeitschrift fr Physik, vol. 163, no. 5, pp. 594-603, 1961.

[187] J. Lambe and C. C. Klick, "Model for luminescence and photoconductivity in the sulfides," Physical Review, vol. 98, no. 4, pp. 909-914, 1955, PR.

[188] H. Arbell and A. Halperin, "Thermoluminescence of $\mathrm{ZnS}$ single crystals," Physical Review, vol. 117, no. 1, pp. 45-52, 1960. 
[189] S. I. Narita and S. Sugiyama, "Some optical properties of zinc sulfide crystals," Journal of the Physical Society of Japan, vol. 20, no. 1, pp. 153-163, 1965.

[190] K. Era, S. Shionoya, and Y. Washizawa, "Mechanism of broad-band luminescences in $\mathrm{ZnS}$ phosphors I. spectrum shift during decay and with excitation intensity," Journal of Physics and Chemistry of Solids, vol. 29, no. 10, pp. 1827-1841, 1968.

[191] K. Matsuura and I. Tsurumi, "Thermoluminescence in $\mathrm{ZnS}$ crystals fired in zinc or sulfur vapor," Journal of the Physical Society of Japan, vol. 39, no. 2, pp. 383-389, 1975.

[192] K. Hirabayashi, H. Kozawaguchi, and B. Tsujiyama, "Study on AC powder EL phosphor deterioration factors," Journal of the Electrochemical Society, vol. 130, no. 11, pp. 2259-63, 1983.

[193] Y. Bacherikov, A. Zhuk, M. Baran, Y. Venger, and N. Korsunska, "Formation of $\mathrm{Cu}$-related emission centers under thermal doping of $\mathrm{ZnS}$ powders with $\mathrm{CuCl}$ and $\mathrm{CuCl}_{2}$," Journal of Luminescence, vol. 165, pp. 94-98, 2015.

[194] C. H. Haake, "Secondary waves of electroluminescence," Journal of Applied Physics, vol. 28 , no. 1 , pp. 117-123, 1957.

[195] G. Destriau, "Brightness waves and transitory phenomena in the quenching of luminescence by alternating electric fields," Journal of Applied Physics, vol. 25, no. 1, pp. 67-71, 1954.

[196] S. Nudelman and F. Matossi, "Electroluminescence with nonsinusoidal fields," Journal of The Electrochemical Society, vol. 101, no. 11, pp. 546-553, 1954.

[197] J. F. Waymouth and F. Bitter, "Experiments on electroluminescence," Phys. Rev., vol. 95, pp. 941-949, 41954.

[198] K. Patek, "Temperature dependence of secondary peak of electroluminescence of ZnS-Cu," Cechoslovackij fiziceskij zurnal, vol. 9, no. 4, pp. 460-467, 1959.

[199] R. Zallen, W. T. Eriksen, and H. Ahlburg, "Electroluminescence under pulsed square wave excitation," Journal of The Electrochemical Society, vol. 107, no. 4, pp. 288$295,1960$.

[200] J. Schanda, "Brightness waves of different activators in $\mathrm{ZnS}$ single crystals," Cechoslovackij fiziceskij zurnal B, vol. 13, no. 2, pp. 147-153, 1963.

[201] L. Sodomka, "The influence of ball milling on the structure and electroluminescence of zinc sulphide powder," Cechoslovackij fiziceskij zurnal B, vol. 14, no. 5, pp. 352-366, 1964. 
[202] R. Bertram, H. Haupt, and H. Nelkowski, "Mechanism of double-comet-shaped electroluminescence in ZnS-Cu crystals," Journal of Luminescence, vol. 16, no. 3, pp. 245-250, 1978.

[203] F. J. Bryant and S. A. Hamid, "Heat treatment of trapping centres in zinc sulphide single crystals," Physica status solidi (a), vol. 2, no. 3, pp. 607-618, 1970.

[204] F. S. Boggess-Machado, "AC frequency dependence of electroluminescent $\mathrm{ZnS}$ phosphor panel color," 2004.

[205] C. J. Winscom, P. G. Harris, and J. Silver, "Equivalent circuits and efficacy of single-layer ACPEL devices," ECS Journal of Solid State Science and Technology, vol. 3, no. 6, R104-R108, 2014.

[206] G. C. Psarras, "Conductivity and dielectric characterization of polymer nanocomposites," in Physical Properties and Applications of Polymer Nanocomposites, Y. W. Mai, Ed. Woodhead Publishing, 2010, pp. 31-69.

[207] G. M. Tsangaris and G. C. Psarras, "The dielectric response of a polymeric threecomponent composite," Journal of Materials Science, vol. 34, no. 9, pp. 21512157, 1999.

[208] C. J. F. Bttcher, O. C. van Belle, P. Bordewijk, and A. Rip, Theory of electric polarization. Elsevier Science Ltd, 1978, vol. 2.

[209] S. Nakamura, G. Sawa, and M. Ieda, "Electrical conduction of nylon 6 at high temperature," Japanese Journal of Applied Physics, vol. 20, no. 1, p. 47, 1981.

[210] N. Fuse, H. Sato, Y. Ohki, and T. Tanaka, "Effects of nanofiller loading on the molecular motion and carrier transport in polyamide," Dielectrics and Electrical Insulation, IEEE Transactions on, vol. 16, no. 2, pp. 524-530, 2009.

[211] G. S. Fulcher, "Analysis of recent measurements of the viscosity of glasses," Journal of the American Ceramic Society, vol. 8, no. 6, pp. 339-355, 1925.

[212] G. Nurk, H. Kasuk, K. Lust, A. Janes, and E. Lust, "Adsorption kinetics of dodecyl sulfate anions on the bismuth (011) plane," Journal of Electroanalytical Chemistry, vol. 553, pp. 1-19, 2003.

[213] E. Warburg, "Ueber die spitzenentladung 2 mittheilung," in Annalen der Physik und Chemie, J. Barth, Ed. 1899, vol. 67, p. 69.

[214] D. Qu, "Studies of the activated carbons used in double-layer supercapacitors," Journal of Power Sources, vol. 109, no. 2, pp. 403-411, 2002. 
[215] D. A. Buchanan, "Scaling the gate dielectric: Materials, integration, and reliability," IBM Journal of Research and Development, vol. 43, no. 3, pp. 245-264, 1999.

[216] I. Soudek, "The effect of irradiation on the dielectric losses of luminescent zinccadmium sulphide," Cechoslovackij fiziceskij zurnal, vol. 7, no. 1, pp. 119-120, 1957.

[217] S. Kronenberg and C. A. Accardo, "Dielectric changes in inorganic phosphors," Physical Review, vol. 101, no. 3, pp. 989-992, 1956.

[218] S. Siddabattuni, T. P. Schuman, and F. Dogan, "Dielectric properties of polymerparticle nanocomposites influenced by electronic nature of filler surfaces," ACS Applied Materials \& Interfaces, vol. 5, no. 6, pp. 1917-1927, 2013.

[219] Y. Y. Chen, J. G. Duh, B. S. Chiou, and C. G. Peng, "Luminescent mechanisms of $\mathrm{ZnS}: \mathrm{Cu}, \mathrm{Cl}$ and $\mathrm{ZnS}: \mathrm{Cu}, \mathrm{Al}$ phosphors," Thin Solid Films, vol. 392, no. 1, pp. 50-55, 2001.

[220] F. Clabau, X. Rocquefelte, T. Le Mercier, P. Deniard, S. Jobic, and M. H. Whangbo, "Formulation of phosphorescence mechanisms in inorganic solids based on a new model of defect conglomeration," Chemistry of Materials, vol. 18, no. 14, pp. 32123220, 2006.

[221] M. E. Orazem, I. Frateur, B. Tribollet, V. Vivier, S. Marcelin, N. Pbre, A. L. Bunge, E. A. White, D. P. Riemer, and M. Musiani, "Dielectric properties of materials showing constant-phase-element (CPE) impedance response," Journal of The Electrochemical Society, vol. 160, no. 6, pp. C215-C225, 2013. 